Water Availability and Use Science Program

\title{
Estimated Use of Water in the United States in 2015
}

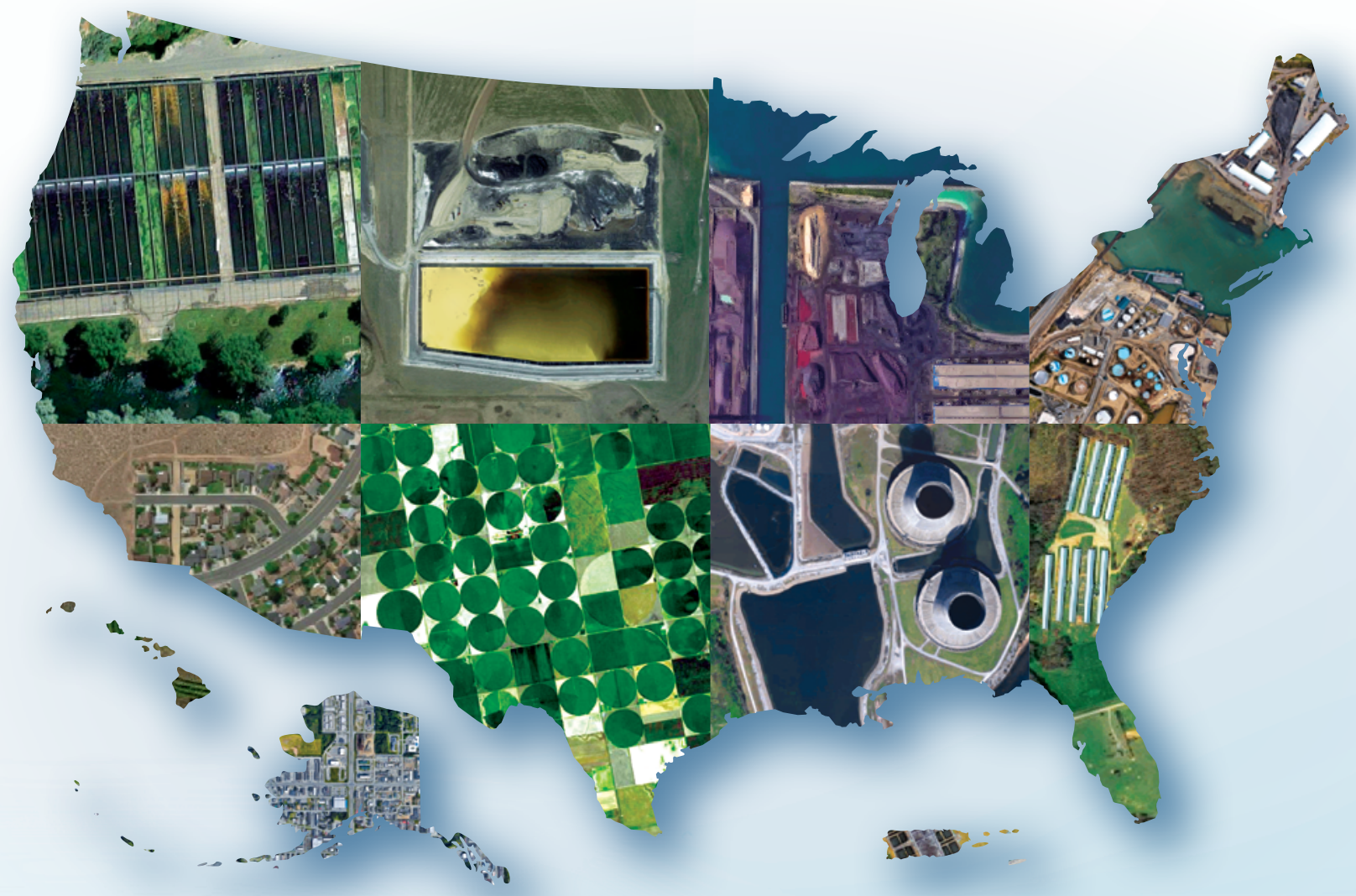

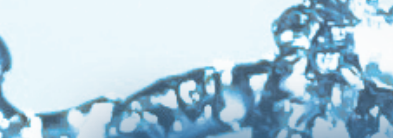

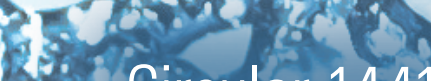
20. Circular 1441

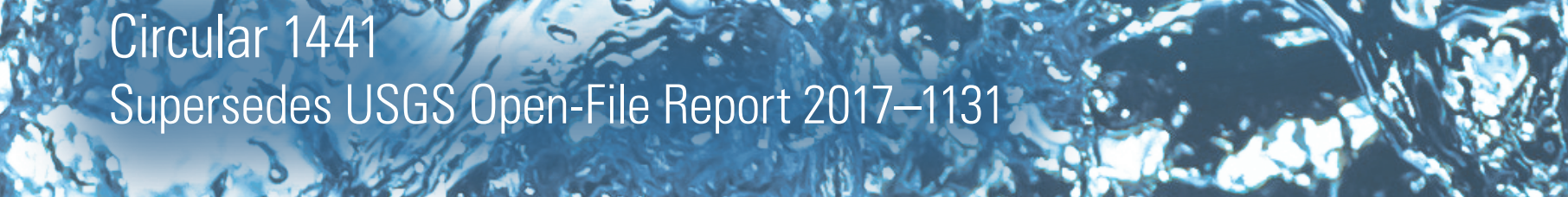

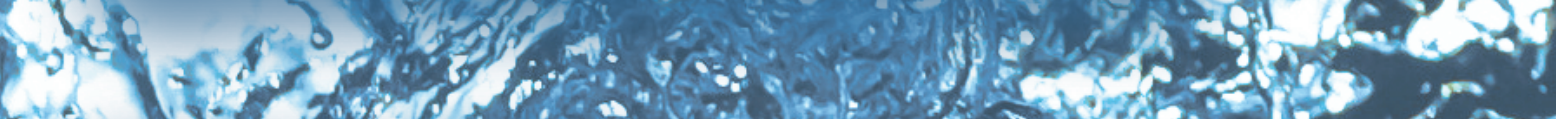
-
A. U. S. Department of the 


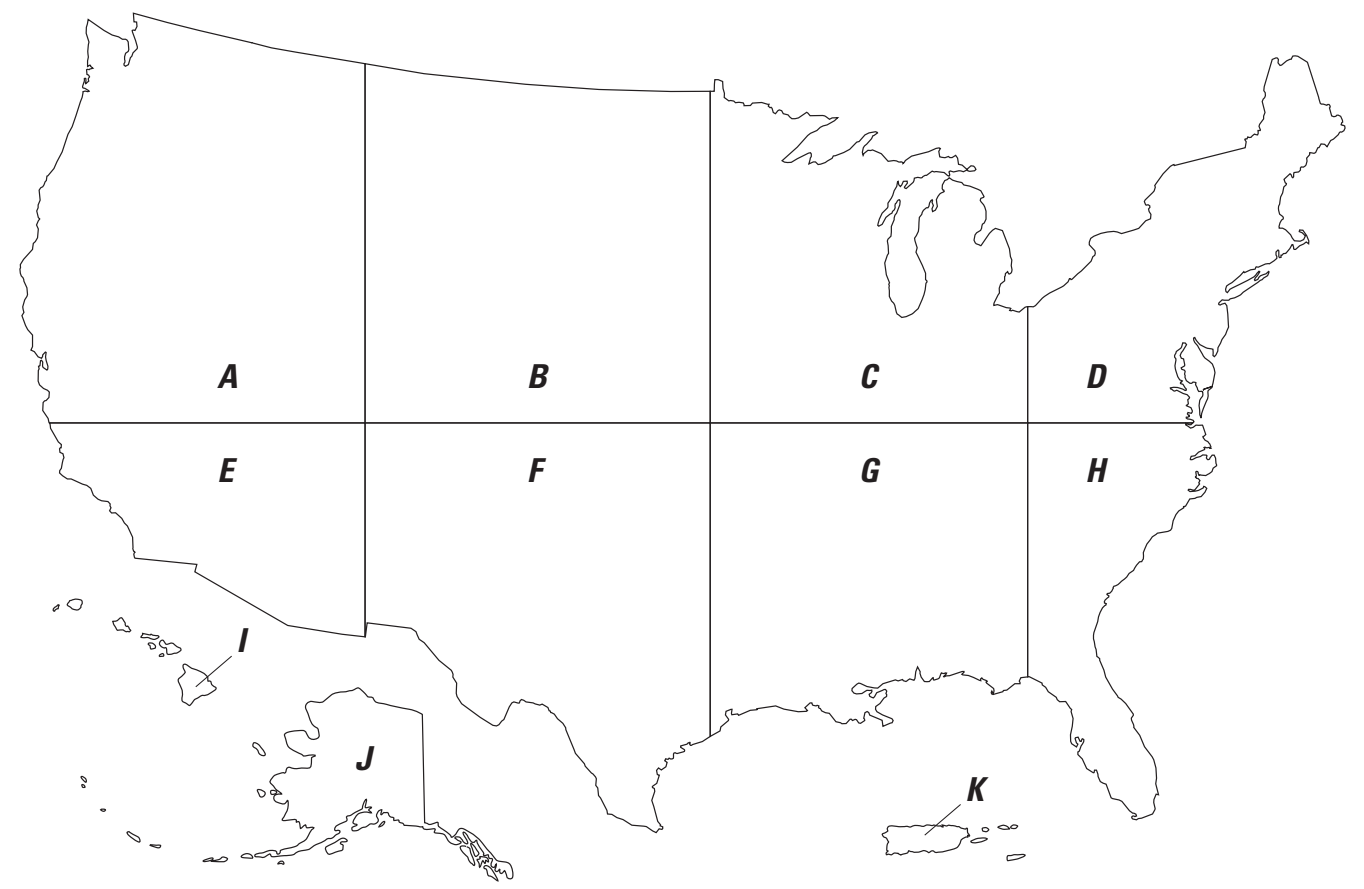

Cover. Composite image. A, Photograph showing aquaculture ponds in Idaho, map data (c) 2018 Google. B, Photograph showing a mine in North Dakota, map data (C) 2018 Google. C, Photograph showing an industrial site along Lake Michigan in Indiana, map data (C) 2018 Google. D, Photograph showing a water treatment facility in Maine, map data (c) 2018 Google. E, Photograph showing irrigated residential landscaping in Nevada, map data (C 2018 Google. $\boldsymbol{F}$, Photograph showing crop irrigation in Kansas, by NASA/GSFC/METI/ERSDAC/JAROS and U.S./Japan ASTER Science Team on June 24, 2001. G, Photograph showing a thermoelectric powerplant in Tennessee, map data (c) 2018 Google. H, Photograph of poultry houses in Georgia, map data (C) 2018 Google. I, Photograph showing agriculture irrigation in Hawaii, map data (C 2018 Google. J, Photograph showing residential sections in Alaska, map data (C) 2018 Google. $\boldsymbol{K}$, Photograph showing a water treatment plant in Puerto Rico, map data () 2018 Google. Background photograph showing splashing water by Simon Buchou, Unsplash on November 20, 2017. Banner photograph showing water drops by Brianna Santellan, Unsplash on October 24, 2017. 


\section{Estimated Use of Water in the United States in 2015}

By Cheryl A. Dieter, Molly A. Maupin, Rodney R. Caldwell, Melissa A. Harris, Tamara I. Ivahnenko, John K. Lovelace, Nancy L. Barber, and Kristin S. Linsey

Water Availability and Use Science Program

Circular 1441

Supersedes USGS Open-File Report 2017-1131 


\title{
U.S. Department of the Interior \\ RYAN K. ZINKE, Secretary
}

\author{
U.S. Geological Survey \\ James F. Reilly II, Director
}

U.S. Geological Survey, Reston, Virginia: 2018
Supersedes USGS Open-File Report 2017-1131

For more information on the USGS - the Federal source for science about the Earth, its natural and living resources, natural hazards, and the environment-visit https://www.usgs.gov or call 1-888-ASK-USGS.

For an overview of USGS information products, including maps, imagery, and publications,

visit https://store.usgs.gov.

Any use of trade, firm, or product names is for descriptive purposes only and does not imply endorsement by the U.S. Government.

Although this information product, for the most part, is in the public domain, it also may contain copyrighted materials as noted in the text. Permission to reproduce copyrighted items must be secured from the copyright owner.

Suggested citation:

Dieter, C.A., Maupin, M.A., Caldwell, R.R., Harris, M.A., Ivahnenko, T.I., Lovelace, J.K., Barber, N.L., and Linsey, K.S., 2018, Estimated use of water in the United States in 2015: U.S. Geological Survey Circular 1441, 65 p., https://doi.org/10.3133/cir1441. [Supersedes USGS Open-File Report 2017-1131.]

ISSN 1067-084X (print) ISSN 2330-5703 (online) ISBN 978-1-4113-4233-0 


\section{Contents}

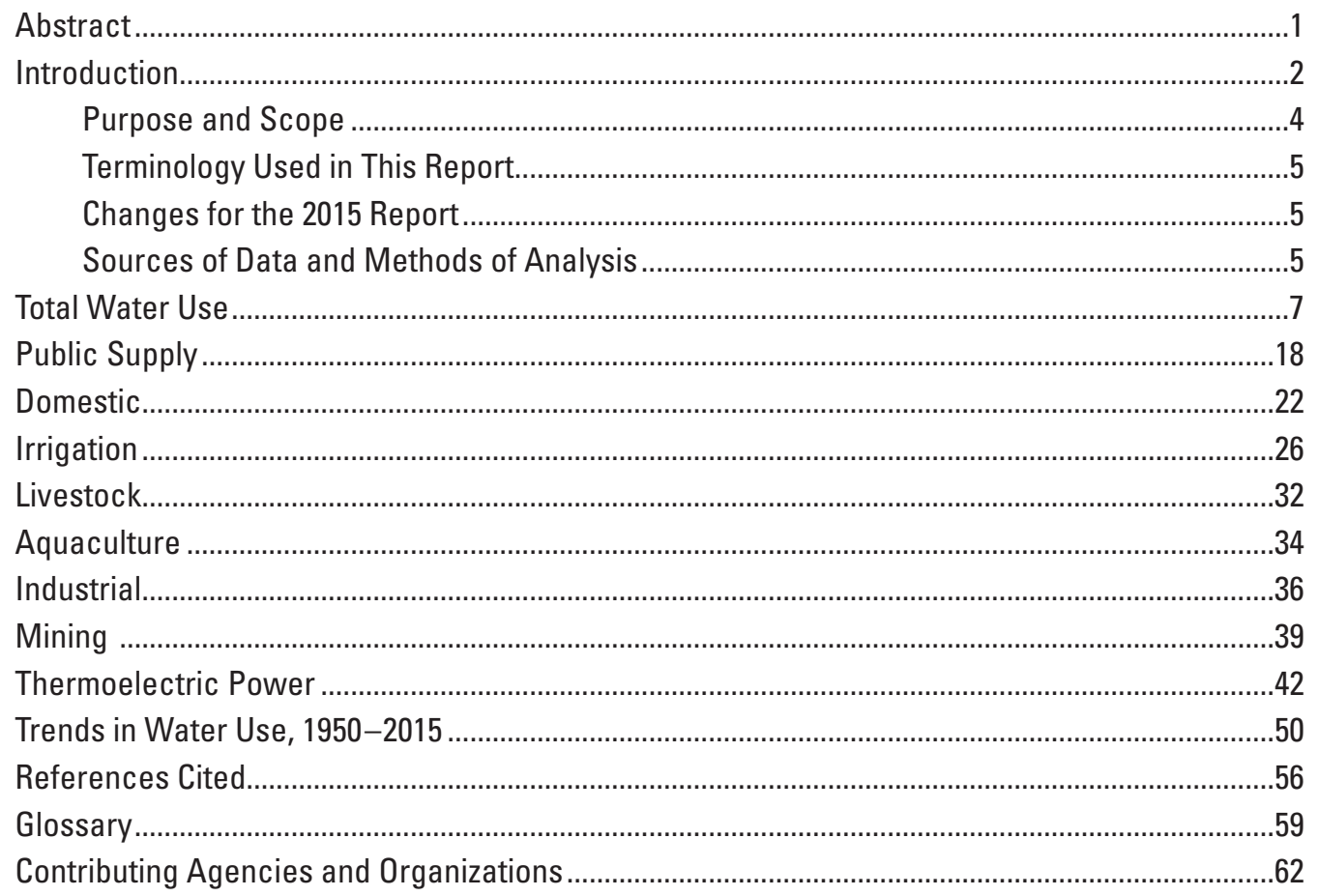

\section{Acknowledgments}

This U.S. Geological Survey (USGS) national compilation of water use would not be possible without the assistance and data provided by the many State and local agencies that manage water resources, operate data-collection programs, and administer regulations for use of water and other natural resources. The agencies and other organizations that provided assistance are listed for each State at the end of this report. The authors also gratefully acknowledge the hard work and dedication of the approximately 45 USGS personnel throughout the Nation who compiled the county-level data for this report. Again, this compilation would not be possible without their assistance. 


\section{Conversion Factors}

U.S. customary units to International System of Units

\begin{tabular}{|c|c|c|}
\hline Multiply & By & To obtain \\
\hline \multicolumn{3}{|c|}{ Length } \\
\hline inch (in.) & 2.54 & centimeter $(\mathrm{cm})$ \\
\hline inch (in.) & 25.4 & millimeter $(\mathrm{mm})$ \\
\hline \multicolumn{3}{|c|}{ Area } \\
\hline acre & 4,047 & square meter $\left(\mathrm{m}^{2}\right)$ \\
\hline acre & 0.4047 & hectare (ha) \\
\hline acre & 0.001562 & square mile $\left(\mathrm{mi}^{2}\right)$ \\
\hline \multicolumn{3}{|c|}{ Volume } \\
\hline acre-foot (acre-ft) & 1,233 & cubic meter $\left(\mathrm{m}^{3}\right)$ \\
\hline acre-foot (acre-ft) & 325,851 & gallon (gal) \\
\hline acre-foot (acre-ft) & 43,560 & cubic foot $\left(\mathrm{ft}^{3}\right)$ \\
\hline cubic foot $\left(\mathrm{ft}^{3}\right)$ & 7.48 & gallon (gal) \\
\hline gallon (gal) & 3.785 & liter (L) \\
\hline gallon (gal) & 3.785 & cubic decimeter $\left(\mathrm{dm}^{3}\right)$ \\
\hline million gallons (Mgal) & 3,785 & cubic meter $\left(\mathrm{m}^{3}\right)$ \\
\hline million gallons (Mgal) & 3.07 & acre-foot (acre-ft) \\
\hline \multicolumn{3}{|c|}{ Flow rate } \\
\hline acre-foot per year (acre-ft/yr) & 1,233 & cubic meter per year $\left(\mathrm{m}^{3} / \mathrm{yr}\right)$ \\
\hline billion gallons per day (Bgal/d) & 1.3815 & billion cubic meters per year \\
\hline gallon per day (gal/d) & 3.785 & liter per day $(\mathrm{L} / \mathrm{d})$ \\
\hline million gallons per day (Mgal/d) & 0.04381 & cubic meter per second $\left(\mathrm{m}^{3} / \mathrm{s}\right)$ \\
\hline million gallons per day (Mgal/d) & 1.547 & cubic foot per second $\left(\mathrm{ft}^{3} / \mathrm{s}\right)$ \\
\hline million gallons per day (Mgal/d) & 1.121 & $\begin{array}{l}\text { thousand acre-foot per year } \\
\quad(\text { acre-ft/yr) }\end{array}$ \\
\hline million gallons per day (Mgal/d) & 1.3815 & million cubic meters per year \\
\hline $\begin{array}{l}\text { thousand acre-feet per year } \\
\text { (acre-ft/yr) }\end{array}$ & 0.8921 & million gallons per day $(\mathrm{Mgal} / \mathrm{d})$ \\
\hline \multicolumn{3}{|c|}{ Energy } \\
\hline gigawatt-hour (gWh) & $3,600,000$ & Megajoule (MJ) \\
\hline kilowatt-hour (kWh) & $3,600,000$ & joule $(\mathrm{J})$ \\
\hline
\end{tabular}

Temperature in degrees Fahrenheit $\left({ }^{\circ} \mathrm{F}\right)$ may be converted to degrees Celsius $\left({ }^{\circ} \mathrm{C}\right)$ as follows:

$$
{ }^{\circ} \mathrm{C}=\left({ }^{\circ} \mathrm{F}-32\right) / 1.8 .
$$




\section{Abbreviations}

$\begin{array}{ll}\text { CONUS } & \text { Contiguous United States } \\ \text { EPA } & \text { U.S. Environmental Protection Agency } \\ \text { ET } & \text { Evapotranspiration } \\ \text { GPCD } & \text { gallons per capita per day } \\ \text { MODIS } & \text { Moderate Resolution Imaging Spectroradiometer } \\ \text { NWC } & \text { National Water Census } \\ \text { NWIS } & \text { National Water Information System } \\ \text { NWUSP } & \text { National Water Use Science Project } \\ \text { SDWIS } & \text { Safe Drinking Water Information System } \\ \text { SECURE } & \text { Science and Engineering to Comprehensively Understand and Responsibly Enhance } \\ \text { SSEBop } & \text { Operational Simplified Surface Energy Balance } \\ \text { USDA } & \text { U.S. Department of Agriculture } \\ \text { USDA NASS } & \text { U.S. Department of Agriculture, National Agricultural Statistics Service } \\ \text { USDOE EIA } & \text { U.S. Department of Energy, Energy Information Administration } \\ \text { USGS } & \text { U.S. Geological Survey } \\ \text { WAUSP } & \text { Water Availability and Use Science Program } \\ \text { WUDR } & \text { U.S. Geological Survey Water Use Data and Research Financial Assistance Program }\end{array}$





\title{
Estimated Use of Water in the United States in 2015
}

\author{
By Cheryl A. Dieter, Molly A. Maupin, Rodney R. Caldwell, Melissa A. Harris, Tamara I. Ivahnenko, \\ John K. Lovelace, Nancy L. Barber, and Kristin S. Linsey
}

\section{Abstract}

Water use in the United States in 2015 was estimated to be about 322 billion gallons per day (Bgal/d), which was 9 percent less than in 2010 . The 2015 estimates put total withdrawals at the lowest level since before 1970, following the same overall trend of decreasing total withdrawals observed from 2005 to 2010. Freshwater withdrawals were $281 \mathrm{Bgal} / \mathrm{d}$, or 87 percent of total withdrawals, and salinewater withdrawals were $41.0 \mathrm{Bgal} / \mathrm{d}$, or 13 percent of total withdrawals. Fresh surface-water withdrawals (198 Bgal/d) were 14 percent less than in 2010, and fresh groundwater withdrawals ( $82.3 \mathrm{Bgal} /$ day) were about 8 percent greater than in 2010. Saline surface-water withdrawals were $38.6 \mathrm{Bgal} / \mathrm{d}$, or 14 percent less than in 2010. Total saline groundwater withdrawals in 2015 were $2.34 \mathrm{Bgal} / \mathrm{d}$, mostly for mining use.

Thermoelectric power and irrigation remained the two largest uses of water in 2015, and total withdrawals decreased for thermoelectric power but increased for irrigation. Withdrawals in 2015 for thermoelectric power were 18 percent less and withdrawals for irrigation were 2 percent greater than in 2010. Similarly, other uses showed reductions compared to 2010 , specifically public supply ( -7 percent), self-supplied domestic ( -8 percent), self-supplied industrial ( -9 percent), and aquaculture ( -16 percent). In addition to irrigation ( 2 percent), mining ( 1 percent) reported larger withdrawals in 2015 than in 2010. Livestock withdrawals remained essentially the same in 2015 compared to 2010 (0 percent change). Thermoelectric power, irrigation, and public-supply withdrawals accounted for 90 percent of total withdrawals in 2015 .

Withdrawals for thermoelectric power were $133 \mathrm{Bgal} / \mathrm{d}$ in 2015 and represented the lowest levels since before 1970. Surface-water withdrawals accounted for more than 99 percent of total thermoelectric-power withdrawals, and 72 percent of those surface-water withdrawals were from freshwater sources. Saline surface-water withdrawals for thermoelectric power accounted for 97 percent of total saline surface-water withdrawals for all uses. Thermoelectric-power withdrawals accounted for 41 percent of total withdrawals for all uses, and freshwater withdrawals for thermoelectric power accounted for 34 percent of the total freshwater withdrawals for all uses. Total consumptive use for thermoelectric power was $4.31 \mathrm{Bgal} / \mathrm{d}$ in 2015 or 3 percent of the total thermoelectric-power withdrawals.

Irrigation withdrawals were $118 \mathrm{Bgal} / \mathrm{d}$ in 2015, an increase of 2 percent from 2010 (116 Bgal/d), but were approximately equal to withdrawals estimated in the 1960s. Irrigation withdrawals, all freshwater, accounted for 42 percent of total freshwater withdrawals for all uses and 64 percent of total freshwater withdrawals for all uses excluding thermoelectric power. Surface-water withdrawals (60.9 Bgal/d) accounted for 52 percent of the total irrigation withdrawals, or about 8 percent less than in 2010. Groundwater withdrawals for irrigation were 57.2 Bgal/d in 2015, about 16 percent more than in 2010 . About 63,500 thousand acres (or 63.5 million acres) were irrigated in 2015, an increase from 2010 of about 1,130 thousand acres ( 2 percent). The number of acres irrigated using sprinkler and microirrigation systems accounted for 63 percent of the total irrigated lands in 2015. Total consumptive use for irrigation was $73.2 \mathrm{Bgal} / \mathrm{d}$ in 2015 or 62 percent of the total use (withdrawals and reclaimed wastewater).

Public-supply withdrawals in 2015 were $39.0 \mathrm{Bgal} / \mathrm{d}$, or 7 percent less than in 2010, continuing the declines observed from 2005 to 2010. Total population in the United States increased from 312.6 million people in 2010 to 325.0 million people in 2015, an increase of 4 percent. Public-supply withdrawals accounted for 14 percent of the total freshwater withdrawals for all uses and 21 percent of freshwater withdrawals for all uses, excluding thermoelectric power. The number of people that received potable water from public-supply facilities in 2015 was 283 million, or about 87 percent of the total United States population. This percentage is 1 percent greater than in 2010. Self-supplied domestic withdrawals were $3.26 \mathrm{Bgal} / \mathrm{d}$, or 8 percent less than in 2010 . More than 98 percent of the self-supplied domestic withdrawals were from groundwater sources.

Self-supplied industrial withdrawals were $14.8 \mathrm{Bgal} / \mathrm{d}$ in 2015, a 9 percent decline from 2010, continuing the downward trend since the peak of $47 \mathrm{Bgal} / \mathrm{d}$ in 1970. Total self-supplied industrial withdrawals were 5 percent of total withdrawals for all uses and 8 percent of total withdrawals for all uses, 
excluding thermoelectric power. Most of the total self-supplied industrial withdrawals were from surface-water sources (82 percent), and nearly all (94 percent) of those surface-water withdrawals were from freshwater sources. Nearly all of the groundwater withdrawals for self-supplied industrial use (98 percent) were from freshwater sources.

Total aquaculture withdrawals were $7.55 \mathrm{Bgal} / \mathrm{d}$ in 2015 , or 16 percent less than in 2010, and surface water was the primary source (79 percent). Most of the surface-water withdrawals occurred at facilities that operated flow-through raceways, which returned the water to the source directly after use. Aquaculture withdrawals accounted for 2 percent of the total withdrawals for all uses and 4 percent of the total withdrawals for all uses, excluding thermoelectric.

Total mining withdrawals in 2015 were $4.00 \mathrm{Bgal} / \mathrm{d}$, or about 1 percent of total withdrawals from all uses and 2 percent of total withdrawals from all uses, excluding thermoelectric. Mining withdrawals increased 1 percent from 2010 to 2015. Groundwater withdrawals accounted for 72 percent of the total mining withdrawals, and most of the groundwater was saline (65 percent). Most (77 percent) of the surface-water withdrawals for mining was freshwater.

Livestock withdrawals in 2015 were $2.00 \mathrm{Bgal} / \mathrm{d}$, the same as in 2010. All livestock withdrawals were from freshwater sources, mostly from groundwater (62 percent). Livestock withdrawals accounted for about 1 percent of total freshwater withdrawals for all uses, excluding thermoelectric power.

In 2015, more than 50 percent of the total withdrawals in the United States were accounted for by 12 States (California, Texas, Idaho, Florida, Arkansas, New York, Illinois, Colorado,
North Carolina, Michigan, Montana, and Nebraska). California accounted for almost 9 percent of the total withdrawals and 9 percent of freshwater withdrawals in the United States, predominantly for irrigation. Texas accounted for almost 7 percent of total withdrawals, predominantly for thermoelectric power, irrigation, and public supply. Florida accounted for 23 percent of the total saline-water withdrawals in the United States, mostly from surface-water sources for thermoelectric power. Texas and California accounted for 59 percent of the total saline groundwater withdrawals in the United States, mostly for mining.

\section{Introduction}

This report, "Estimated use of water in the United States in 2015," is the 14th in a series of U.S. Geological Survey (USGS) Circular reports that have been published every 5 years since 1950 . The 65 -year span of national reports represents the longest compilation record of water-use data by a Federal agency in the United States. Estimates of withdrawals enable the depiction of trends in total water use for the Nation among different geographic areas, categories of use, and sources over time. The USGS is dedicated to providing reliable scientific information that accurately describes current and historical conditions and enables a better understanding of the Earth's water resources. Water-use information is a critical component of water budgets, which are essential to surface-water and groundwater availability studies. This

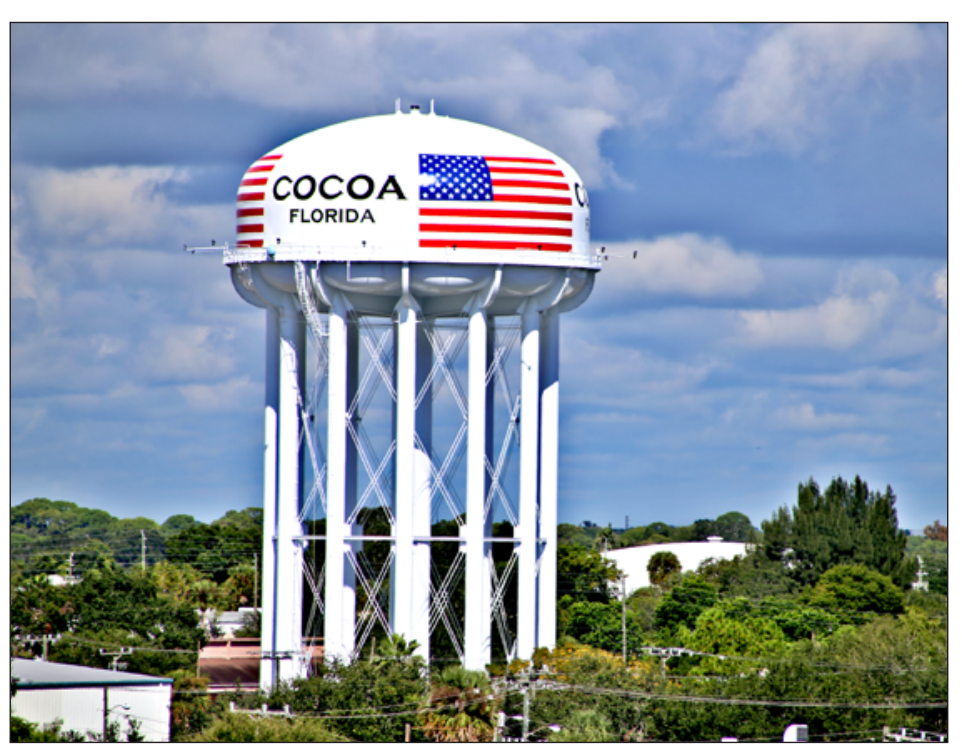

Public supply water tank, winner of the "2015 Tank of the Year" presented by Tnemec Company, Inc. Photograph provided by the City of Cocoa, Florida, used with permission.

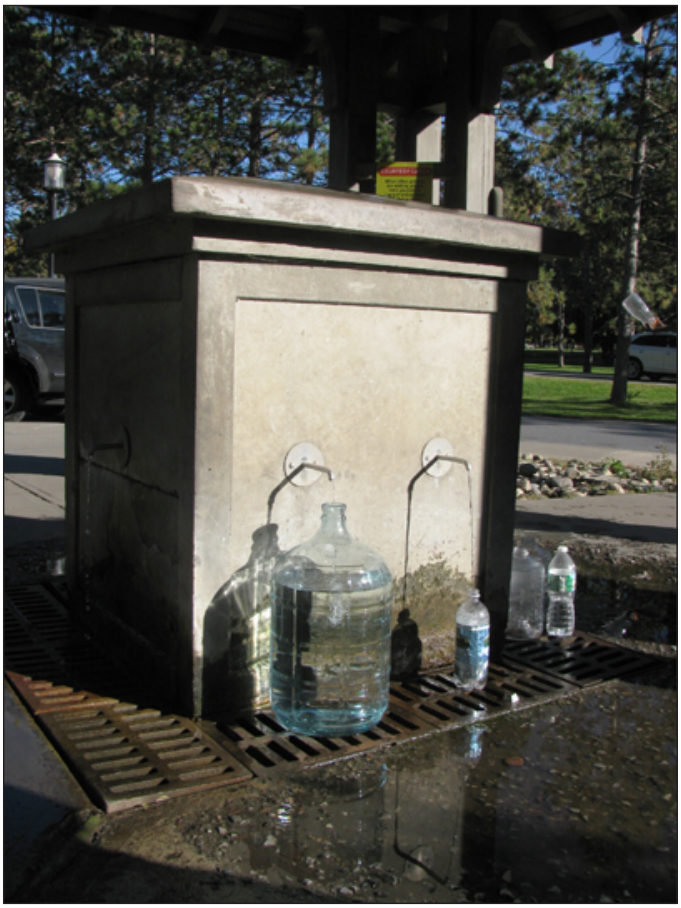

State Seal Spring is used for domestic drinking water in Saratoga County, New York. Photograph by Tamara Ivahnenko, USGS. 
information is also essential to accurately understand how future water demands will be met while maintaining adequate water quality and quantities for human and ecosystem needs in the United States.

The USGS National Water Use Science Project (NWUSP), a component of the Water Availability and Use Science Program (WAUSP), facilitates the 5-year compilation of water use estimates for the United States as part of the National Water Census (NWC). The NWC, implemented as part of the SECURE (Science and Engineering to Comprehensively Understand and Responsibly Enhance) Water Act (Subtitle F of Public Law 111-11, the Omnibus Public Land Management Act), provides data and tools designed to support water managers in the accurate assessment of water availability at regional and national scales (https://water.usgs.gov/ watercensus/). Over time, the NWUSP has met various challenges in estimating water use in the United States (https://water.usgs.gov/watuse/) and as a result has reduced some data collections over time to address limitations of available resources for analysis and limitations of capabilities for accurate interpolations.

USGS NWC-supported projects with direct relevance to water use and the NWUSP 2015 compilation efforts are focused on several categories of water use, including thermoelectric power and public supply. For thermoelectric power, thermoelectric withdrawals in the United States for 2010 acquired from three Federal datasets were evaluated and compared (Harris and Diehl, 2017). For public supply, work continues to construct a site-specific database of public-supply withdrawal, distribution, use, and return data for each State. The NWUSP is in the process of developing methods and tools to estimate groundwater withdrawals from major aquifer systems for every county in the United States.

The SECURE Water Act authorized a program to support water-use and availability activities related to data collection and methods research and development at the State level. The USGS Water-Use Data and Research program (WUDR) began to provide financial assistance through cooperative agreements with State water-resource agencies in 2015 (https://water.usgs. gov/wausp/wudr/index.html). The goal of the USGS WUDR is to improve water-use data for the United States at the State level, which will allow better, more accurate estimates of water use in the United States and provide information needed to manage water resources, and (or) forecast future water-use needs. The WUDR Program promotes cooperative work with the State agencies. Data from funded projects will be incorporated into USGS databases.

Data dissemination capabilities and data-collection efforts have improved over the course of each 5-year compilation. The online resource, "USGS Water Use Data for the Nation," (https://waterdata.usgs.gov/nwis/wu) provides the best available county water-use data (1985-2015). These county-level estimates are the foundation for the statewide totals presented in each 5-year compilation report and are stored, updated, and disseminated using the USGS National Water Information System (NWIS) database. Data are retrievable as county,

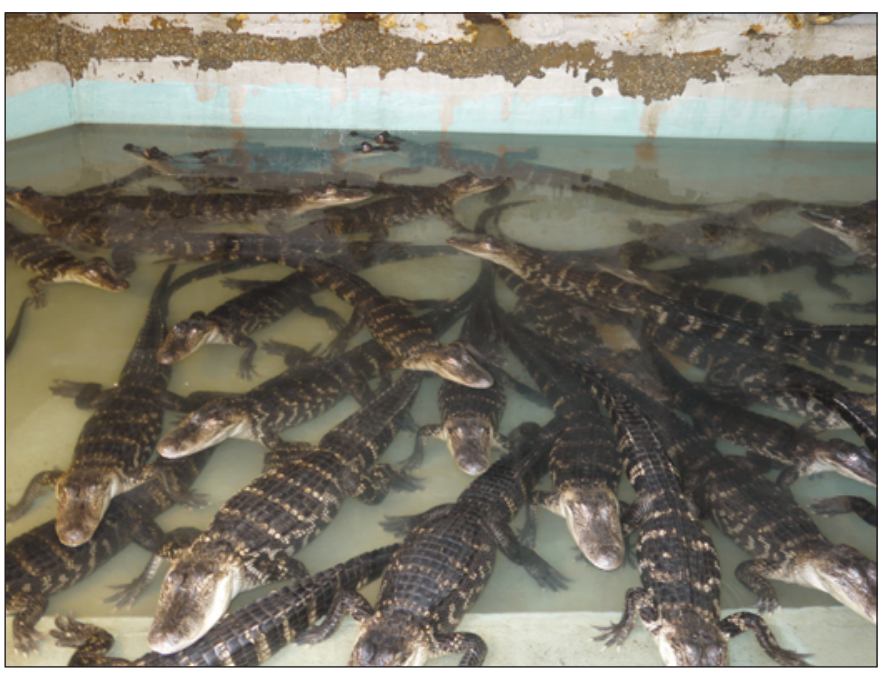

Alligators in a holding pool, St. Tammany Parish, Louisiana, is an example of aquaculture water use. Photograph by Pierre Sargent, USGS.

State, and national totals for each category of use as reported in the 5-year compilation reports. Because data are updated periodically and revised during interim years, the website can enable quick and easy access to the most current water-use data. A companion data release of 2015 county-level data is available through the USGS ScienceBase catalog at https://doi. org/10.5066/F7TB15V5 (Dieter and others, 2018).

Factors such as demographics, new manufacturing and cooling-system technologies, economic trends, legal decisions, and climatic fluctuations have varying effects on water use. Between 2010 and 2015, population in the United States increased 4 percent, or approximately 12 million people, from 313 million people in 2010 to 325 million people in 2015 . The Southern and Western States had the largest estimated increases in population from 2010 to 2015 as a result of large estimated increases in population in Texas, California, and Florida (U.S. Census Bureau, 2017). A few Eastern States had estimated decreases in population (Puerto Rico, West Virginia, and Vermont) (U.S. Census Bureau, 2017). Population growth puts additional pressure on existing public utilities and increases demand on sometimes already limited water supplies. In parts of the United States, communities have sought additional water sources or instituted waterconservation measures to meet increasing demands. New cooling-system technologies and wastewater-management practices at thermoelectric powerplants and industrial facilities are examples of water-saving practices that are being implemented. Powerplants have reduced the demand for cooling water by implementing more efficient cooling systems, such as changing to recirculating systems (also referred to as recirculation systems) or building new plants with dry-cooling systems. Industrial facilities are using more efficient waterconserving manufacturing technologies, driven by the need to reduce costs associated with water/energy production needs. Increases in industrial reuse and recycling of wastewater help to reduce withdrawals from the available resources and treated discharges to surface waters over time. 
Climate fluctuations affect water use, particularly for irrigation, power generation, and public supply. According to the National Oceanic and Atmospheric Administration's Annual Climate Report for 2015, the contiguous United States (CONUS) experienced average annual air temperatures 2.4 degrees Fahrenheit above the 20th century average in every State, and precipitation was 4.53 inches greater than the 20th century average (National Oceanic and Atmospheric Administration, 2016a). The winter temperature was greater than the average for the CONUS. The winter was abnormally cold in the Northeast and Midwest and warm in the West. Boston, Massachusetts, received a record 110.6 inches of snow. Fourteen States in central and southeastern CONUS experienced wetter than normal conditions, including Texas and Oklahoma, both of which had record wet years, relieving them of drought conditions that began in 2010. Florida experienced record warm temperatures 3.3 degrees Fahrenheit greater than the long-term average. California's near-record warm temperatures and low precipitation in the winter caused drought conditions, including low reservoir levels in the State (National Oceanic and Atmospheric Administration National Centers for Environmental Information, 2016a).

\section{Purpose and Scope}

This report presents average daily withdrawals (in millions of gallons per day, Mgal/d) for calendar year 2015, by source (groundwater and surface water) and quality (fresh and saline) for the 50 States, the District of Columbia, Puerto Rico, and the U.S. Virgin Islands (hereafter referred to as "States" for brevity). Withdrawals are reported by category of use: public supply, domestic (including self-supplied domestic and deliveries from public supply), irrigation, livestock, aquaculture, self-supplied industrial (referred to as "industrial" for brevity), mining, and self-supplied thermoelectric power (referred to as "thermoelectric power" for brevity). Saline water is defined as water containing dissolved solids of 1,000 milligrams per liter or more. All withdrawals for the public supply, domestic, irrigation, and livestock categories are reported as totals, although in some areas water is treated to reduce salinity for these uses. Aquaculture totals include a small amount of saline surface-water withdrawals for four States. Both freshwater and saline-water withdrawals are reported for industrial, mining, and thermoelectric-power uses. In addition to withdrawals, average daily consumptiveuse estimates (in Mgal/d) for irrigation and thermoelectric power for 2015 are presented in this report.

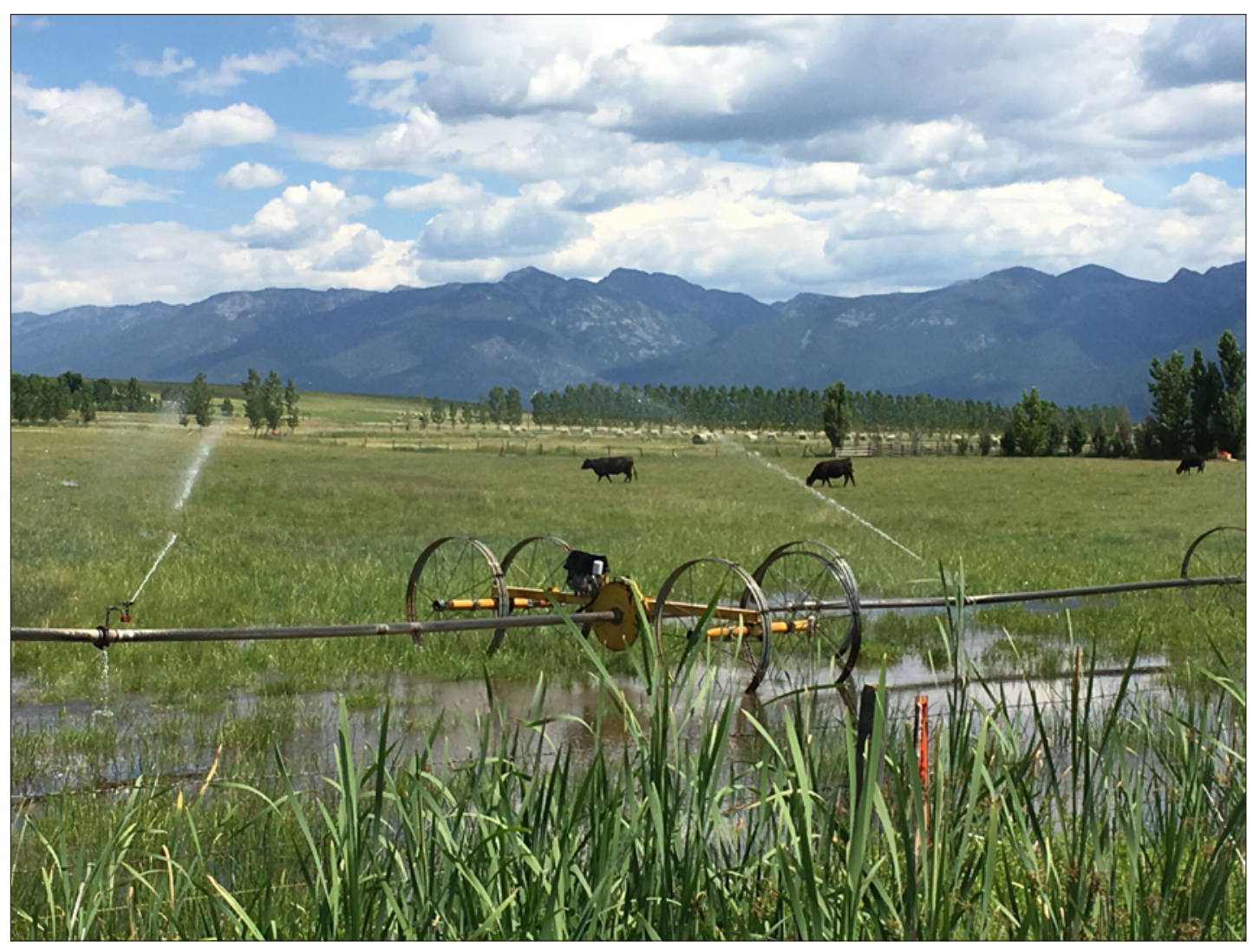

Irrigation in Montana. Photograph by Rodney Caldwell, USGS. 
The series of 5-year national water-use estimates compiled by the USGS serves as one of the few sources of information about regional and national trends in water withdrawals. These historical reports (MacKichan, 1951, 1957; MacKichan and Kammerer, 1961; Murray, 1968; Murray and Reeves, 1972, 1977; Solley and others, 1983, 1988, 1993, 1998; Hutson and others, 2004; Kenny and others, 2009; Maupin and others, 2014) are available online at https:// water.usgs.gov/watuse/50years.html. Statewide data between 1950 and 2015 produced for the 5-year national water-use estimates are available online at https://waterdata.usgs.gov/ nwis/wu. County-level data are available only for 1985-2015 from the same website.

\section{Terminology Used in This Report}

A glossary of the terms and units used in this report is located at the end of the report and is available online at https://water.usgs.gov/watuse/wuglossary.html. Terms and units depicting withdrawals and ancillary data for the 5-year compilations have not changed since 2000. Withdrawal for each category of use represents the total amount of water removed from the water source for a particular use. Additional water may be used for the category from public-supply deliveries or from reclaimed wastewater. In most cases, some fraction of the total withdrawal will be returned to a water source after use and will be available for other subsequent uses. Estimates of reclaimed wastewater use were compiled by some States for the industrial, thermoelectric-power, and irrigation categories. Available reclaimed wastewater use data are included in the tables and graphics for the thermoelectricpower and irrigation categories, but not for industrial because of the small volumes of water compared to the totals and the incomplete reporting across the Nation.

The amount of water that is not readily available for another use because it is evaporated, transpired, incorporated into products or crops, consumed by livestock or humans, or otherwise removed from the immediate water environment is termed consumptive use. Estimates of return flows and consumptive use were discontinued after 1995, primarily because of resource and data constraints. This report reinstates the consumptive-use estimates for the thermoelectric-power and irrigation categories.

Rates of water use (withdrawals, deliveries from public supply, consumptive use) are expressed in terms of Mgal/d and thousands of acre-feet per year (acre-ft/yr). The term billion of gallons per day (Bgal/d) is used in the "Abstract" and "Trends in Water Use" sections of this report to more simply express large numbers for total uses. Units of million or billion of gallons per day do not represent actual daily rates, but rather are used to express total amounts as an average daily rate over a single year (annually). Water demands fluctuate seasonally and may be very different between hot summer months and cold winter months. Therefore, withdrawal estimates in this report represent the total annual withdrawals averaged over 365 days.
Water-use values and population data are rounded to three significant figures, except for population values in the "Trends in Water Use" section, which are rounded to four significant figures. All values are rounded independently, so the sums of individual rounded numbers may not equal the totals. The percentage of changes discussed in the text are calculated from the unrounded data and are expressed as integers. In discussions of States that compose the majority of withdrawals for a given category, the State names are listed in order of decreasing magnitude of withdrawals.

\section{Changes for the 2015 Report}

A matrix showing the different categories of use and how the terminology has changed over time is available online at https://water.usgs.gov/watuse/WU-Category-Changes.html. Links to definitions of water-use categories are included in the matrix. This report includes the same categories of use that were reported in 2005 and 2010, and every category of use includes data from every State. Deliveries from public supply for domestic use were again compiled in 2015, but public-supply deliveries for commercial, industrial, and thermoelectric-power uses were not compiled for all States. This report reinstates consumptive-use estimates for the thermoelectric power and irrigation categories, which were discontinued after 1995, primarily because of resource and data constraints.

Data were not compiled for hydrologic units (watersheds). Data were not compiled for commercial water use, hydroelectric-power generation, wastewater treatment (returns), consumptive use (except for the categories of thermoelectric power and irrigation), or irrigation conveyance losses. Some of these additional data may have been collected for individual States but are not compiled as a national dataset or included in this report.

The "Trends in Water Use" section of this report includes national totals for withdrawals by category of use and source of water from 1950 to 2015 . Totals have changed for some categories and years because of revisions to individual State data during interim years. Because of these revisions, some of the percentage changes in this report will be slightly different from data published previously by Maupin and others (2014).

\section{Sources of Data and Methods of Analysis}

Data presented in this report were compiled from various sources, depending on the category of use and the information available for each State. USGS personnel in each State determined the best sources of information available, then compiled or estimated the data and prepared documentation of the sources and methods used to determine the water-use totals. Data in this report may have been derived from reported, estimated, or calculated means using different 


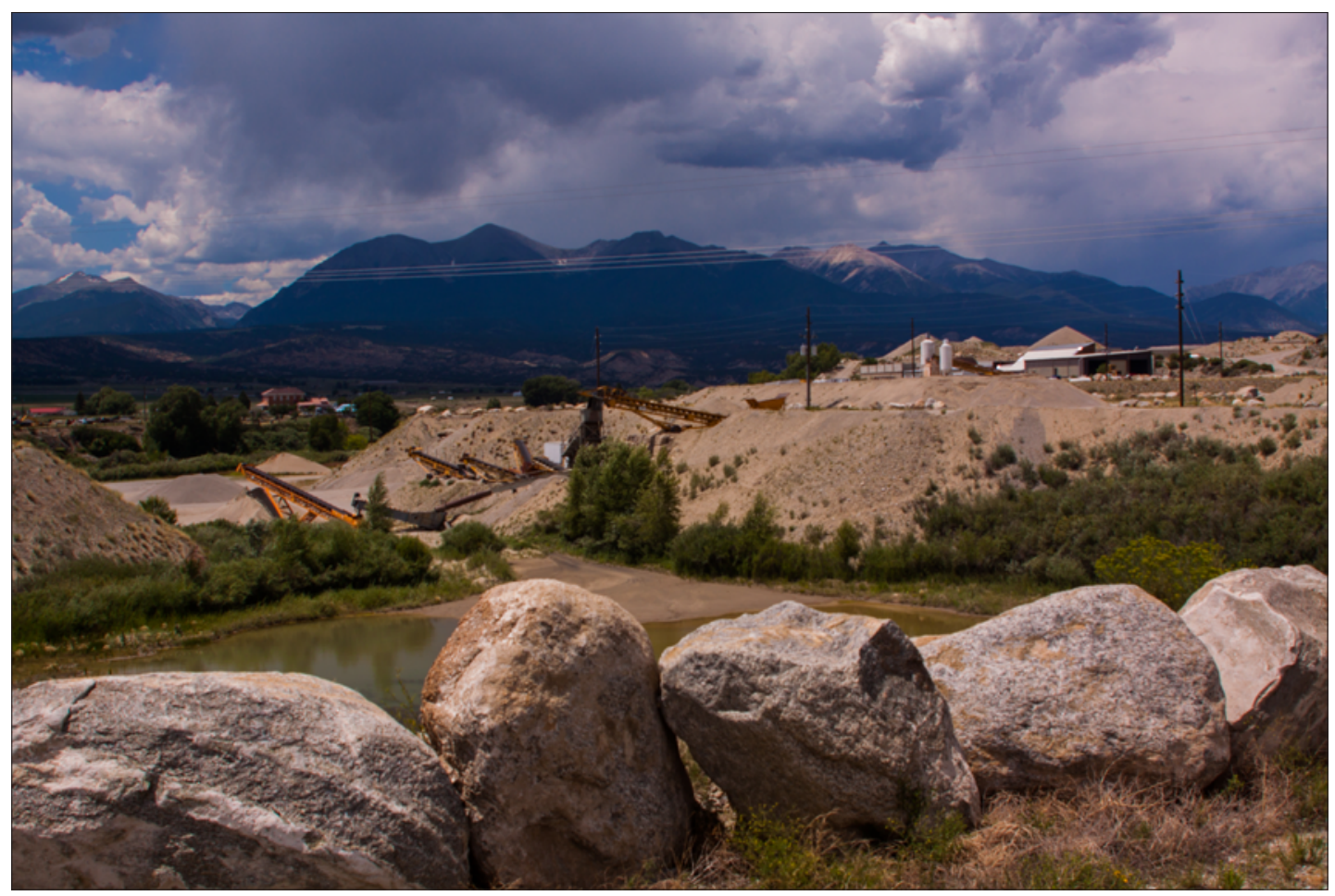

Mining in Chaffee County, Colorado. Photograph by Robert Stogner, used with permission.

sources and methods and, therefore, will have varying levels of accuracy. Because the largest users and the most prominent categories of use within each State have the greatest effect on the totals, obtaining reliable information for these large users and categories was the primary focus of the compilation effort.

Sources of information used in the compilation include national datasets, State agency data, and local contacts. National datasets available to each State include U.S. Census Bureau population estimates (U.S. Census Bureau, 2016), U.S. Department of Agriculture (USDA) Farm and Ranch Irrigation Survey, USDA Census of Agriculture, USDA National Agricultural Statistics Service (NASS) crop and livestock estimates, USDA Cropland Data Layer, and U.S. Department of Energy, Energy Information Administration (USDOE EIA) facility reports.

In addition to the nationally available datasets listed above, category-specific data were compiled and (or) estimated by the NWUSP and provided to USGS personnel to help refine estimates for thermoelectric power, irrigation, livestock, mining, and aquaculture. For thermoelectric power, cooling-system classifications for powerplants, modeled withdrawals, and consumptive use were provided from internal USGS sources (Diehl and others, 2013; Diehl and Harris, 2014) using USDOE EIA and project ancillary data. To estimate irrigation consumptive use, estimates of evapotranspiration derived from 1-kilometer scale Moderate Resolution Imaging Spectroradiometer (MODIS) satellite data using the Operational Simplified Surface Energy Balance (SSEBop) model (Senay and others, 2013) were provided for each State.
Datasets and sources of information used to produce the national estimates for the livestock, aquaculture, and mining categories include the USDA NASS, USDOE EIA, FracFocus Chemical Disclosure Registry, USGS National Minerals Information Center, and various State agencies responsible for oil and gas development or underground-injection control programs. Sources of information are discussed in greater detail in the individual category sections of this report.

Many of these supporting data, such as those from USDA NASS and USDOE EIA, are collected annually. Other data are provided for years other than 2015 but were used to develop the 2015 estimates in some States because they were the most complete data available. For example, the USDA Census of Agriculture is produced in years ending in 2 and 7, and the USDA Farm and Ranch Irrigation Survey is produced in years ending in 3 and 8. Correlation of water-use data in this report with specific climatic conditions for 2015 is not recommended because data for years other than 2015 may have been used to develop some water-use estimates.

Guidelines for preparing the 2015 water-use estimates were distributed to USGS contact personnel through workshops, web-based seminars, and written documents. These guidelines have been published as USGS Open-File Report 2017-1029, "Guidelines for preparation of State water-use estimates for 2015" (Bradley, 2017), available at https:/doi.org/ 10.3133/ofr20171029. Reports published for individual States by USGS offices as part of the NWUSP, as well as a list of contact personnel, also are available online at https:/water. usgs.gov/watuse/. 
Total water withdrawals in the United States for 2015 were estimated for eight categories of use: public supply, self-supplied domestic, irrigation, livestock, aquaculture, industrial, mining, and thermoelectric power (fig. 1). The three largest categories were thermoelectric power, irrigation, and public supply, accounting for 90 percent of the national total. The remaining categories of self-supplied domestic, industrial, aquaculture, mining, and livestock together were 10 percent of total water withdrawals estimated in this report.

Total estimates of State populations and withdrawals by source for 2015 are listed in table 1 . The total estimated population in the United States in 2015 was 325 million, and total freshwater and saline-water withdrawals were estimated to be $322,000 \mathrm{Mgal} / \mathrm{d}$, or 361,000 thousand acre-ft/yr. Freshwater withdrawals of $281,000 \mathrm{Mgal} / \mathrm{d}$ made up 87 percent of the total, and saline-water withdrawals made up the remaining 13 percent $(41,000 \mathrm{Mgal} / \mathrm{d})$. Most freshwater withdrawals were used for irrigation, and most saline-water withdrawals were seawater and brackish coastal water used for thermoelectric power. Total surface-water withdrawals were estimated to be $237,000 \mathrm{Mgal} / \mathrm{d}$, or 74 percent of total withdrawals. About 84 percent $(198,000 \mathrm{Mgal} / \mathrm{d})$ of total surface-water withdrawals were freshwater. Total groundwater withdrawals were 84,700 Mgal/d, of which 97 percent $(82,300 \mathrm{Mgal} / \mathrm{d})$ was freshwater.

Total withdrawals by category and State are listed in table $2 A$, in million gallons per day, and in table $2 B$, in thousand acre-feet per year. A geographic representation of total withdrawals in the United States is shown in figure 2 with a stacked bar chart that illustrates a west to east representation of withdrawals by category and State. Total surface-water and groundwater, and total freshwater and saline-water, withdrawals by State are shown in figure 3 .

Withdrawals for thermoelectric power were the largest nationwide (tables $2 A$ and $2 B$ ) and are predominant in the east, whereas irrigation withdrawals are greatest in the west (fig. 2). Total withdrawals for thermoelectric power (133,000 Mgal/d) are mostly derived from freshwater sources ( 72 percent), and total freshwater withdrawals for thermoelectric power accounted for 34 percent of the total freshwater withdrawals for all categories nationwide. Total saline-water withdrawals for thermoelectric power accounted for about 92 percent of total saline-water withdrawals for all categories nationwide. Irrigation withdrawals (all freshwater) totaled 118,000 Mgal/d and accounted for 42 percent of total freshwater withdrawals for all categories nationwide. Total withdrawals for public supply (39,000 Mgal/d, and 99 percent freshwater) represented nearly 14 percent of the total freshwater withdrawals for all categories nationwide (table 2).

In 2015 , more than 50 percent of the total withdrawals in the United States were accounted for by 12 States: California, Texas, Idaho, Florida, Arkansas, New York, Illinois, Colorado, North Carolina, Michigan, Montana, and Nebraska. Total withdrawals in California accounted for about 9 percent of the total withdrawals for all categories nationwide. California's freshwater withdrawals were 9 percent of total freshwater withdrawals for all categories nationwide, predominantly for irrigation. Total withdrawals in Texas accounted for about 7 percent of total withdrawals for all categories nationwide, and its freshwater withdrawals accounted for about 7 percent of total freshwater withdrawals for all categories nationwide, predominantly for thermoelectric. Idaho's freshwater withdrawals for irrigation were the second largest nationwide, and aquaculture withdrawals (all freshwater) were the largest nationwide. Combined, these two categories of use in Idaho accounted for 6 percent of total freshwater withdrawals nationwide. Florida had the largest saline withdrawals and accounted for 23 percent of total saline withdrawals for all categories nationwide; Florida's saline surface-water withdrawals were used mostly for thermoelectric power. Florida, New York, and Maryland accounted for more than 50 percent of total saline surface-water withdrawals nationwide, predominantly for thermoelectric power. Texas and California accounted for about 60 percent of the total saline groundwater withdrawals nationwide, mostly for mining.

Water withdrawals by use and State are listed for surface water in tables $3 A$ and $3 B$ and for groundwater in tables $4 A$ and $4 B$. In 2015, more surface water than groundwater was withdrawn for all categories of use except domestic, livestock, and mining; however, irrigation is nearly evenly split between surface water and groundwater. Thermoelectric power accounted for about 48 percent of the total fresh surface-water withdrawals and irrigation accounted for about 31 percent. The largest surface-water withdrawals were in Texas, Idaho, Florida, California, New York, and North Carolina, cumulatively accounting for about 29 percent of total surface-water withdrawals for all categories nationwide. Large quantities of fresh surface water were withdrawn for thermoelectric power in Texas, Illinois, Michigan, and Alabama. Large saline surface-water withdrawals for thermoelectric power occurred in Florida, New York, Maryland, and New Jersey, which cumulatively accounted for 61 percent of the national total saline surface-water withdrawals.

Of the total fresh groundwater withdrawals nationwide $(82,300 \mathrm{Mgal} / \mathrm{d})$, irrigation accounted for 70 percent, primarily in California, Arkansas, Nebraska, Idaho, and Texas. Fresh groundwater irrigation withdrawals in these five States cumulatively accounted for 46 percent of the total fresh groundwater withdrawals for all categories nationwide. Nearly all groundwater withdrawals (97 percent) were from freshwater, predominantly used for irrigation. Saline groundwater withdrawals were predominantly used for mining (80 percent) and occurred in Texas, California, and Oklahoma. Irrigation used greater than three times more fresh groundwater than public supply, which was the next largest use of fresh groundwater in the Nation. 


\section{Public supply, 12 percent}

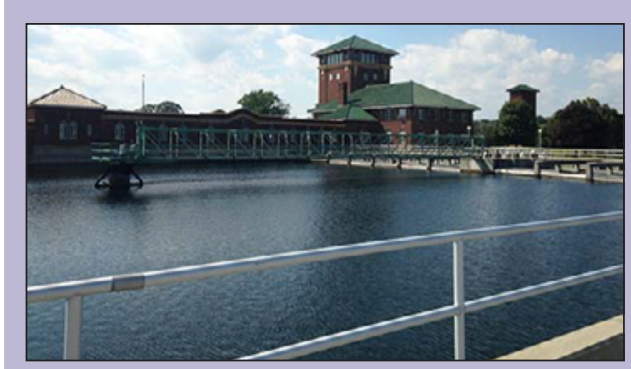

Water treatment plant, Baltimore, Maryland

\section{Irrigation, 37 percent}

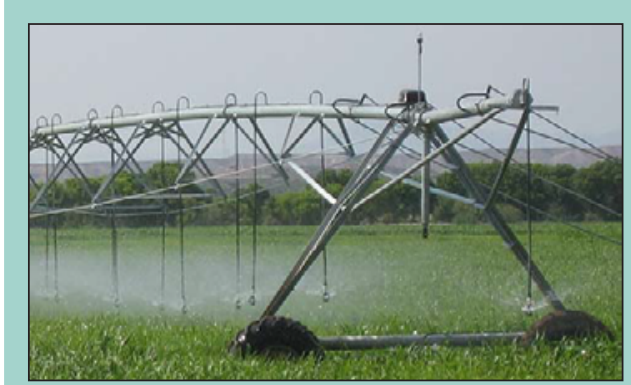

Sprinkler irrigation of corn field, Greenlee County, Arizona

\section{Aquaculture, 2 percent}

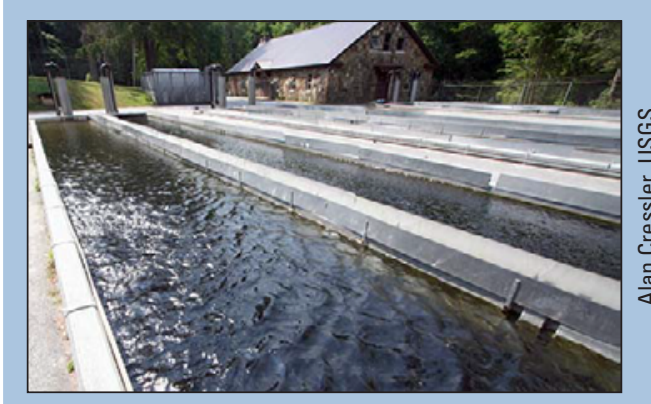

Trout hatchery, Oconee County, South Carolina

\section{Mining, 1 percent}

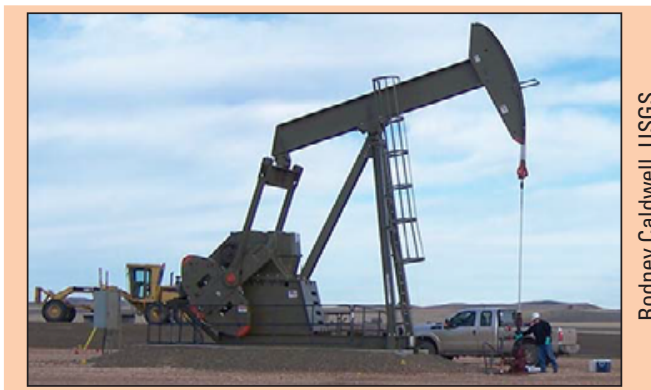

Pumpjack in Richland County, Montana

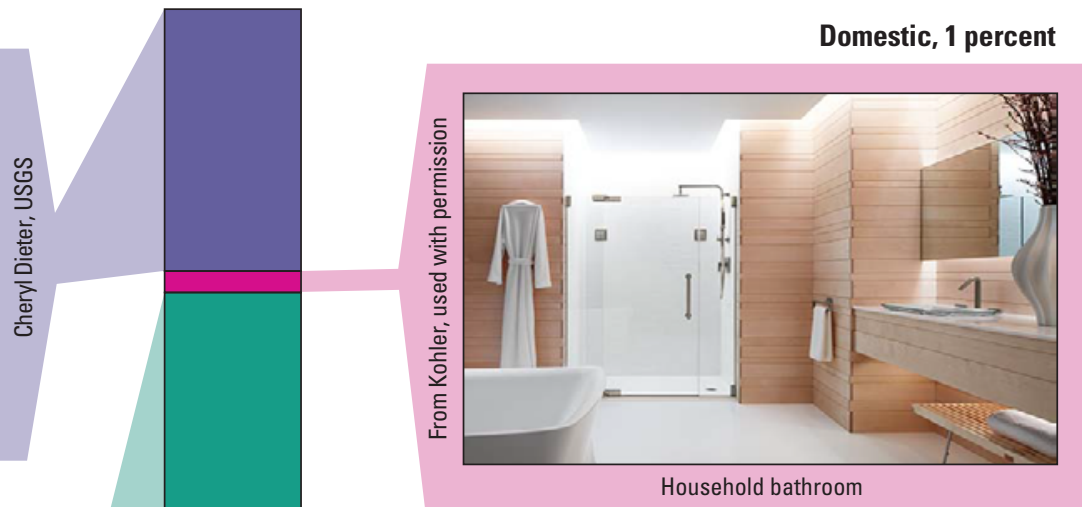

Livestock, 1 percent

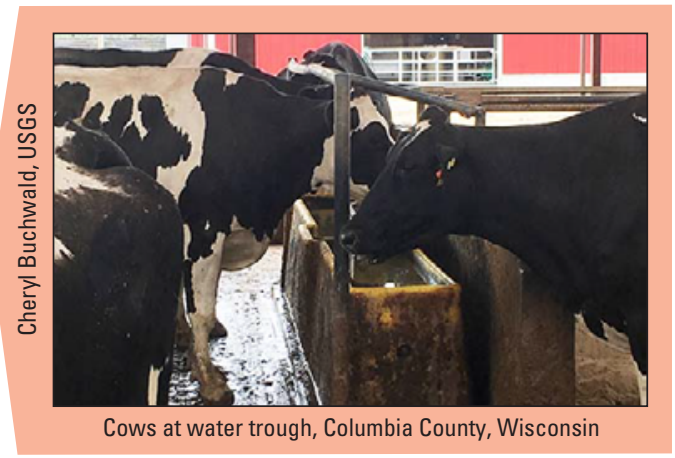

Industrial, 5 percent

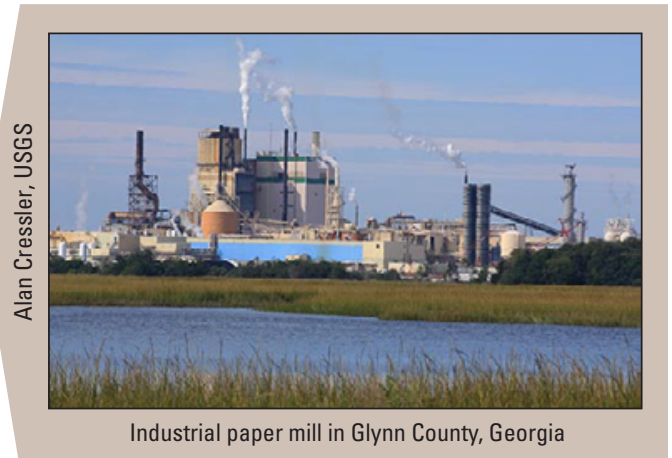

Thermoelectric power, 41 percent

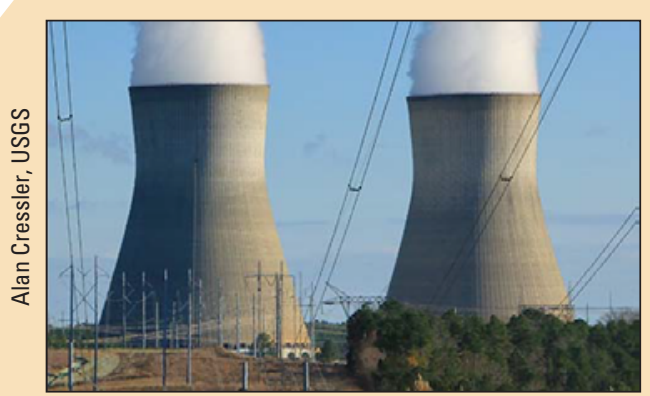

Thermoelectric powerplant, Burke County, Georgia

Figure 1. Total water withdrawals by category, 2015 . 
Table 1. Total water withdrawals by source and State, 2015.

[Values may not sum to totals because of independent rounding]

\begin{tabular}{|c|c|c|c|c|c|c|c|c|c|c|c|c|c|}
\hline \multirow{4}{*}{ State } & \multirow{4}{*}{$\begin{array}{l}\text { Population } \\
\text { (thousands) }\end{array}$} & \multicolumn{6}{|c|}{$\begin{array}{c}\text { Withdrawals } \\
\text { (million gallons per day) }\end{array}$} & & & & \multicolumn{3}{|c|}{$\begin{array}{c}\text { Withdrawals } \\
\text { (thousand acre-feet per year) }\end{array}$} \\
\hline & & \multicolumn{6}{|c|}{ By source and type } & \multirow{2}{*}{\multicolumn{3}{|c|}{ Total }} & \multirow{2}{*}{\multicolumn{3}{|c|}{ Total }} \\
\hline & & \multicolumn{3}{|c|}{ Groundwater } & \multicolumn{3}{|c|}{ Surface water } & & & & & & \\
\hline & & Fresh & Saline & Total & Fresh & Saline & Total & Fresh & Saline & Total & Fresh & Saline & Total \\
\hline Alabama ............................ & 4,860 & 501 & 0 & 501 & 7,750 & 0 & 7,750 & 8,250 & 0 & 8,250 & 9,250 & 0 & 9,250 \\
\hline 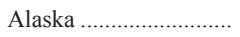 & 738 & 226 & 89.2 & 315 & 408 & 43.2 & 451 & 633 & 132 & 766 & 710 & 148 & 858 \\
\hline 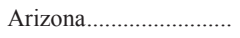 & 6,830 & 2,760 & 0 & 2,760 & 3,220 & 0 & 3,220 & 5,980 & 0 & 5,980 & 6,700 & 0 & 6,700 \\
\hline 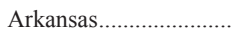 & 2,980 & 9,590 & 0 & 9,590 & 4,250 & 0 & 4,250 & 13,800 & 0 & 13,800 & 15,500 & 0 & 15,500 \\
\hline California ....................... & 39,100 & 17,100 & 359 & 17,400 & 8,540 & 2,800 & 11,300 & 25,600 & 3,160 & 28,800 & 28,700 & 3,550 & 32,200 \\
\hline 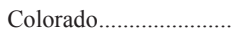 & 5,460 & 1,500 & 24.2 & 1,530 & 8,800 & 0 & 8,800 & 10,300 & 24.2 & 10,300 & 11,500 & 27.1 & 11,600 \\
\hline Connecticut ..................... & 3,590 & 128 & 0 & 128 & 489 & 2,510 & 3,000 & 617 & 2,510 & 3,130 & 691 & 2,820 & 3,510 \\
\hline 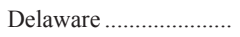 & 946 & 170 & 0 & 170 & 364 & 256 & 620 & 534 & 256 & 790 & 598 & 287 & 886 \\
\hline District of Columbia ... & 672 & 0 & 0 & 0 & 0.05 & 0 & 0.05 & 0.05 & 0 & 0.05 & 0.06 & 0 & 0.06 \\
\hline Florida ............................. & 20,300 & 3,580 & 198 & 3,770 & 2,110 & 9,400 & 11,500 & 5,690 & 9,600 & 15,300 & 6,370 & 10,800 & 17,100 \\
\hline 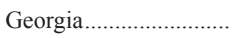 & 10,200 & 1,150 & 0 & 1,150 & 2,130 & 102 & 2,230 & 3,280 & 102 & 3,380 & 3,680 & 114 & 3,790 \\
\hline 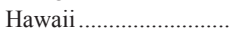 & 1,430 & 338 & 20.2 & 359 & 344 & 357 & 702 & 683 & 377 & 1,060 & 766 & 423 & 1,190 \\
\hline 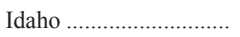 & 1,650 & 5,350 & 0 & 5,350 & 12,400 & 0 & 12,400 & 17,700 & 0 & 17,700 & 19,900 & 0 & 19,900 \\
\hline 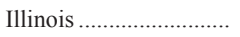 & 12,900 & 870 & 21.0 & 891 & 9,600 & 0 & 9,600 & 10,500 & 21.0 & 10,500 & 11,700 & 23.5 & 11,800 \\
\hline 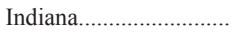 & 6,620 & 699 & 0 & 699 & 6,480 & 0 & 6,480 & 7,180 & 0 & 7,180 & 8,050 & 0 & 8,050 \\
\hline 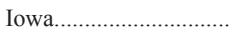 & 3,120 & 630 & 0 & 630 & 2,060 & 0 & 2,060 & 2,690 & 0 & 2,690 & 3,010 & 0 & 3,010 \\
\hline 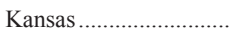 & 2,910 & 2,840 & 6.18 & 2,840 & 1,180 & 0 & 1,180 & 4,010 & 6.18 & 4,020 & 4,500 & 6.93 & 4,510 \\
\hline 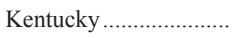 & 4,430 & 207 & 0 & 207 & 2,630 & 0 & 2,630 & 2,830 & 0 & 2,830 & 3,180 & 0 & 3,180 \\
\hline Louisiana............................ & 4,670 & 1,740 & 0 & 1,740 & 6,740 & 261 & 7,000 & 8,480 & 261 & 8,750 & 9,510 & 293 & 9,800 \\
\hline 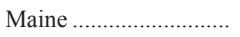 & 1,330 & 84.8 & 0 & 84.8 & 300 & 124 & 424 & 385 & 124 & 509 & 431 & 139 & 570 \\
\hline 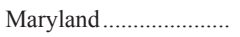 & 6,010 & 295 & 0 & 295 & 935 & 5,300 & 6,230 & 1,230 & 5,300 & 6,530 & 1,380 & 5,940 & 7,320 \\
\hline Massachusetts .............. & 6,790 & 380 & 0 & 380 & 540 & 488 & 1,030 & 920 & 488 & 1,410 & 1,030 & 547 & 1,580 \\
\hline Michigan ........................ & 9,920 & 766 & 0.58 & 767 & 9,290 & 0 & 9,290 & 10,100 & 0.58 & 10,100 & 11,300 & 0.65 & 11,300 \\
\hline Minnesota......................... & 5,490 & 776 & 0 & 776 & 2,450 & 0 & 2,450 & 3,230 & 0 & 3,230 & 3,620 & 0 & 3,620 \\
\hline Mississippi .................... & 2,990 & 2,240 & 12.9 & 2,260 & 427 & 2.07 & 429 & 2,670 & 15.0 & 2,690 & 2,990 & 16.8 & 3,010 \\
\hline 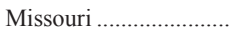 & 6,080 & 1,740 & 0 & 1,740 & 6,690 & 0 & 6,690 & 8,430 & 0 & 8,430 & 9,450 & 0 & 9,450 \\
\hline Montana .......................... & 1,030 & 188 & 16.3 & 205 & 9,610 & 0 & 9,610 & 9,800 & 16.3 & 9,810 & 11,000 & 18.3 & 11,000 \\
\hline 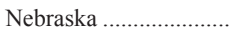 & 1,900 & 5,810 & 6.41 & 5,820 & 3,680 & 0 & 3,680 & 9,490 & 6.41 & 9,500 & 10,600 & 7.19 & 10,600 \\
\hline Nevada ............................ & 2,890 & 1,360 & 82.2 & 1,440 & 1,520 & 0 & 1,520 & 2,880 & 82.2 & 2,960 & 3,230 & 92.2 & 3,320 \\
\hline New Hampshire ............ & 1,330 & 80.4 & 0 & 80.4 & 162 & 693 & 855 & 242 & 693 & 935 & 271 & 777 & 1,050 \\
\hline New Jersey ..................... & 8,960 & 569 & 0 & 569 & 1,310 & 3,430 & 4,740 & 1,880 & 3,430 & 5,310 & 2,110 & 3,840 & 5,950 \\
\hline New Mexico.................. & 2,090 & 1,350 & 89.4 & 1,440 & 1,460 & 0 & 1,460 & 2,810 & 89.4 & 2,900 & 3,150 & 100 & 3,250 \\
\hline New York ......................... & 19,800 & 890 & 0.95 & 890 & 4,420 & 5,480 & 9,910 & 5,310 & 5,480 & 10,800 & 5,960 & 6,150 & 12,100 \\
\hline North Carolina .............. & 10,000 & 520 & 0 & 520 & 8,400 & 1,360 & 9,750 & 8,920 & 1,360 & 10,300 & 10,000 & 1,520 & 11,500 \\
\hline North Dakota.................... & 757 & 187 & 15.1 & 202 & 1,190 & 0 & 1,190 & 1,380 & 15.1 & 1,400 & 1,550 & 16.9 & 1,560 \\
\hline 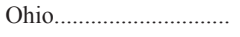 & 11,600 & 866 & 0 & 866 & 5,660 & 0 & 5,660 & 6,520 & 0 & 6,520 & 7,310 & 0 & 7,310 \\
\hline Oklahoma ........................ & 3,910 & 960 & 155 & 1,110 & 848 & 0 & 848 & 1,810 & 155 & 1,960 & 2,030 & 173 & 2,200 \\
\hline Oregon............................ & 4,030 & 1,480 & 0 & 1,480 & 5,100 & 0 & 5,100 & 6,580 & 0 & 6,580 & 7,370 & 0 & 7,370 \\
\hline Pennsylvania ................. & 12,800 & 622 & 5.60 & 628 & 5,410 & 0 & 5,410 & 6,030 & 5.60 & 6,040 & 6,760 & 6.28 & 6,770 \\
\hline Rhode Island .................. & 1,060 & 32.7 & 0 & 32.7 & 88.8 & 222 & 311 & 122 & 222 & 343 & 136 & 249 & 385 \\
\hline South Carolina .............. & 4,900 & 365 & 0 & 365 & 5,810 & 0 & 5,810 & 6,170 & 0 & 6,170 & 6,920 & 0 & 6,920 \\
\hline South Dakota.................... & 881 & 238 & 0 & 238 & 162 & 0 & 162 & 400 & 0 & 400 & 448 & 0 & 448 \\
\hline Tennessee ......................... & 6,600 & 430 & 0 & 430 & 5,990 & 0 & 5,990 & 6,420 & 0 & 6,420 & 7,200 & 0 & 7,200 \\
\hline 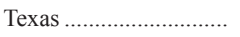 & 27,500 & 6,170 & 1,030 & 7,200 & 12,700 & 1,360 & 14,100 & 18,900 & 2,390 & 21,300 & 21,200 & 2,670 & 23,800 \\
\hline 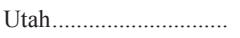 & 3,000 & 1,050 & 93.2 & 1,150 & 2,820 & 257 & 3,080 & 3,880 & 350 & 4,230 & 4,340 & 392 & 4,740 \\
\hline 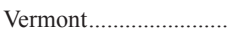 & 626 & 36.7 & 0 & 36.7 & 54.2 & 0 & 54.2 & 90.9 & 0 & 90.9 & 102 & 0 & 102 \\
\hline 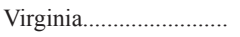 & 8,380 & 284 & 14.3 & 298 & 4,030 & 2,400 & 6,430 & 4,310 & 2,420 & 6,730 & 4,830 & 2,710 & 7,540 \\
\hline Washington....................... & 7,170 & 1,530 & 0 & 1,530 & 2,730 & 0 & 2,730 & 4,260 & 0 & 4,260 & 4,770 & 0 & 4,770 \\
\hline West Virginia................. & 1,840 & 130 & 4.57 & 134 & 2,190 & 0 & 2,190 & 2,320 & 4.57 & 2,320 & 2,600 & 5.12 & 2,600 \\
\hline Wisconsin..................... & 5,770 & 772 & 0 & 772 & 4,980 & 0 & 4,980 & 5,760 & 0 & 5,760 & 6,450 & 0 & 6,450 \\
\hline Wyoming ......................... & 586 & 652 & 96.8 & 748 & 7,400 & 0 & 7,400 & 8,050 & 96.8 & 8,140 & 9,020 & 108 & 9,130 \\
\hline Puerto Rico.................... & 3,470 & 120 & 0 & 120 & 549 & 1,700 & 2,250 & 669 & 1,700 & 2,370 & 750 & 1,910 & 2,660 \\
\hline U.S. Virgin Islands ...... & 104 & 2.67 & 0 & 2.67 & 2.70 & 99.9 & 103 & 5.37 & 99.9 & 105 & 6.02 & 112 & 118 \\
\hline TOTAL & 325,000 & 82,300 & 2,340 & 84,700 & 198,000 & 38,600 & 237,000 & 281,000 & 41,000 & 322,000 & 315,000 & 45,900 & 361,000 \\
\hline
\end{tabular}


Table 2A. Total water withdrawals by water-use category, 2015, in million gallons per day.

[Values may not sum to totals because of independent rounding. Values for public supply and aquaculture include fresh and saline-water withdrawals]

\begin{tabular}{|c|c|c|c|c|c|c|c|c|c|c|c|c|c|c|}
\hline \multirow[t]{2}{*}{ State } & \multirow{2}{*}{$\begin{array}{c}\text { Public } \\
\text { supply }\end{array}$} & \multirow{2}{*}{$\begin{array}{c}\text { Domes- } \\
\text { tic }\end{array}$} & \multirow{2}{*}{$\begin{array}{c}\begin{array}{c}\text { Irriga- } \\
\text { tion }\end{array} \\
\text { Fresh } \\
\end{array}$} & \multirow{2}{*}{$\begin{array}{l}\begin{array}{l}\text { Live- } \\
\text { stock }\end{array} \\
\text { Fresh } \\
\end{array}$} & \multirow{2}{*}{$\begin{array}{c}\begin{array}{c}\text { Aqua- } \\
\text { culture }\end{array} \\
\text { Total }\end{array}$} & \multicolumn{2}{|c|}{ Industrial } & \multicolumn{2}{|c|}{ Mining } & \multicolumn{2}{|c|}{$\begin{array}{c}\text { Thermoelectric } \\
\text { power }\end{array}$} & \multicolumn{3}{|c|}{ Total } \\
\hline & & & & & & Fresh & Saline & Fresh & Saline & Fresh & Saline & Fresh & Saline & Total \\
\hline Alabama .............................. & 762 & 36.7 & 223 & 26.2 & 49.4 & 494 & 0 & 30.2 & 0 & 6,630 & 0 & 8,250 & 0 & 8,250 \\
\hline 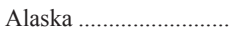 & 99.2 & 11.4 & 1.52 & 0.13 & 410 & 8.35 & 1.83 & 36.4 & 131 & 66.7 & 0 & 633 & 132 & 766 \\
\hline 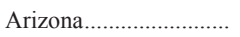 & 1,200 & 24.0 & 4,530 & 38.9 & 34.5 & 6.12 & 0 & 68.3 & 0 & 83.5 & 0 & 5,980 & 0 & 5,980 \\
\hline 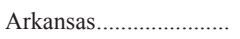 & 363 & 12.8 & 11,600 & 34.1 & 251 & 157 & 0 & 3.07 & 0 & 1,440 & 0 & 13,800 & 0 & 13,800 \\
\hline California ........................ & 5,150 & 127 & 19,000 & 183 & 727 & 399 & 0 & 45.8 & 272 & 36.4 & 2,840 & 25,600 & 3,160 & 28,800 \\
\hline 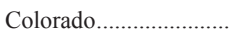 & 844 & 35.4 & 9,000 & 33.3 & 260 & 84.1 & 0 & 7.70 & 24.2 & 37.2 & 0 & 10,300 & 24.2 & 10,300 \\
\hline Connecticut ..................... & 240 & 30.8 & 11.3 & 1.15 & 25.2 & 181 & 41.6 & 4.25 & 0 & 126 & 2,470 & 617 & 2,510 & 3,130 \\
\hline 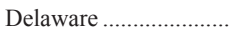 & 86.4 & 14.5 & 113 & 1.34 & 1.98 & 302 & 0 & 0.65 & 0 & 14.4 & 256 & 534 & 256 & 790 \\
\hline District of Columbia ... & 0 & 0 & 0.05 & 0 & 0 & 0 & 0 & 0 & 0 & 0 & 0 & 0.05 & 0 & 0.05 \\
\hline 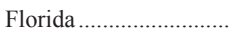 & 2,380 & 177 & 2,450 & 26.1 & 15.0 & 245 & 1.08 & 130 & 0 & 434 & 9,420 & 5,690 & 9,600 & 15,300 \\
\hline 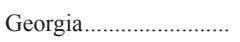 & 1,070 & 104 & 738 & 44.9 & 85.1 & 475 & 0 & 19.8 & 0 & 741 & 102 & 3,280 & 102 & 3,380 \\
\hline 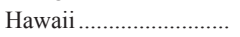 & 267 & 8.10 & 385 & 1.61 & 18.6 & 0.24 & 0 & 0.92 & 0 & 1.48 & 377 & 683 & 377 & 1,060 \\
\hline 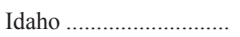 & 276 & 70.2 & 15,300 & 50.8 & 1,960 & 57.6 & 0 & 23.1 & 0 & 1.79 & 0 & 17,700 & 0 & 17,700 \\
\hline 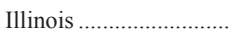 & 1,480 & 92.1 & 234 & 36.2 & 10.7 & 431 & 0 & 55.0 & 21.0 & 8,140 & 0 & 10,500 & 21.0 & 10,500 \\
\hline 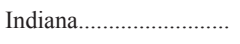 & 628 & 127 & 133 & 39.2 & 14.5 & 2,290 & 0 & 126 & 0 & 3,820 & 0 & 7,180 & 0 & 7,180 \\
\hline 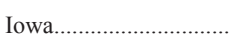 & 390 & 32.0 & 35.0 & 165 & 19.7 & 288 & 0 & 75.5 & 0 & 1,680 & 0 & 2,690 & 0 & 2,690 \\
\hline 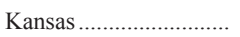 & 351 & 17.7 & 2,680 & 104 & 6.39 & 38.1 & 0 & 5.99 & 0 & 817 & 0 & 4,010 & 6.18 & 4,020 \\
\hline 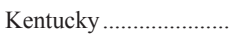 & 553 & 22.1 & 39.6 & 40.8 & 48.4 & 225 & 0 & 40.6 & 0 & 1,860 & 0 & 2,830 & 0 & 2,830 \\
\hline 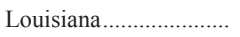 & 709 & 39.3 & 1,050 & 6.35 & 493 & 2,140 & 0 & 6.24 & 0 & 4,040 & 261 & 8,480 & 261 & 8,750 \\
\hline 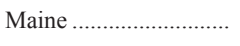 & 85.0 & 31.6 & 18.9 & 2.05 & 54.0 & 182 & 42.9 & 6.33 & 0 & 5.30 & 80.7 & 385 & 124 & 509 \\
\hline Maryland ........................... & 750 & 114 & 64.1 & 8.00 & 7.98 & 49.4 & 1.37 & 16.8 & 0 & 220 & 5,300 & 1,230 & 5,300 & 6,530 \\
\hline Massachusetts .............. & 648 & 35.1 & 139 & 1.03 & 9.93 & 27.9 & 0 & 9.60 & 0 & 50.1 & 487 & 920 & 488 & 1,410 \\
\hline Michigan .......................... & 1,030 & 187 & 332 & 23.7 & 73.8 & 518 & 0 & 85.7 & 0.58 & 7,800 & 0 & 10,100 & 0.58 & 10,100 \\
\hline Minnesota.......................... & 515 & 82.2 & 276 & 58.9 & 16.5 & 259 & 0 & 9.20 & 0 & 2,010 & 0 & 3,230 & 0 & 3,230 \\
\hline Mississippi ...................... & 400 & 48.1 & 1,770 & 17.1 & 127 & 182 & 0 & 9.45 & 6.58 & 118 & 8.42 & 2,670 & 15.0 & 2,690 \\
\hline Missouri ............................ & 797 & 57.5 & 1,370 & 63.7 & 164 & 85.2 & 0 & 29.6 & 0 & 5,860 & 0 & 8,430 & 0 & 8,430 \\
\hline 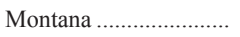 & 153 & 23.7 & 9,450 & 42.2 & 17.1 & 9.67 & 0 & 21.6 & 16.3 & 75.7 & 0 & 9,800 & 16.3 & 9,810 \\
\hline Nebraska ........................... & 275 & 19.0 & 6,090 & 110 & 26.5 & 44.3 & 0 & 9.60 & 6.41 & 2,920 & 0 & 9,490 & 6.41 & 9,500 \\
\hline 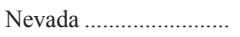 & 531 & 35.8 & 2,070 & 4.94 & 34.0 & 5.71 & 0 & 195 & 11.3 & 8.73 & 70.9 & 2,880 & 82.2 & 2,960 \\
\hline New Hampshire ............ & 95.5 & 29.7 & 5.20 & 0.84 & 17.2 & 12.6 & 0 & 6.13 & 0 & 74.8 & 693 & 242 & 693 & 935 \\
\hline New Jersey ....................... & 1,180 & 90.7 & 93.9 & 0.88 & 9.78 & 94.1 & 0 & 58.3 & 0 & 361 & 3,430 & 1,880 & 3,430 & 5,310 \\
\hline New Mexico................... & 262 & 24.6 & 2,370 & 32.0 & 24.1 & 3.40 & 0 & 56.8 & 89.4 & 33.5 & 0 & 2,810 & 89.4 & 2,900 \\
\hline New York ......................... & 2,420 & 187 & 53.5 & 25.7 & 60.4 & 312 & 8.43 & 40.2 & 0.95 & 2,210 & 5,470 & 5,310 & 5,480 & 10,800 \\
\hline North Carolina .............. & 938 & 169 & 325 & 66.5 & 1,000 & 193 & 0 & 38.3 & 0 & 6,180 & 1,360 & 8,920 & 1,360 & 10,300 \\
\hline North Dakota.................... & 84.2 & 3.69 & 233 & 20.8 & 5.32 & 19.6 & 0 & 30.7 & 15.1 & 983 & 0 & 1,380 & 15.1 & 1,400 \\
\hline 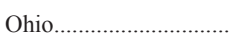 & 1,310 & 139 & 55.0 & 24.6 & 39.3 & 348 & 0 & 129 & 0 & 4,480 & 0 & 6,520 & 0 & 6,520 \\
\hline 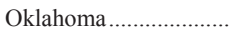 & 611 & 30.3 & 931 & 70.6 & 3.29 & 52.0 & 0 & 37.4 & 155 & 71.7 & 0 & 1,810 & 155 & 1,960 \\
\hline Oregon............................ & 567 & 73.9 & 5,160 & 16.3 & 634 & 105 & 0 & 11.3 & 0 & 11.4 & 0 & 6,580 & 0 & 6,580 \\
\hline Pennsylvania ................. & 1,390 & 208 & 34.3 & 39.5 & 96.0 & 645 & 0 & 38.1 & 5.60 & 3,580 & 0 & 6,030 & 5.60 & 6,040 \\
\hline Rhode Island ................ & 97.5 & 6.57 & 4.25 & 0.12 & 6.81 & 2.05 & 0.03 & 2.92 & 0 & 1.33 & 222 & 122 & 222 & 343 \\
\hline South Carolina .............. & 633 & 118 & 126 & 9.87 & 6.69 & 286 & 0 & 10.1 & 0 & 4,980 & 0 & 6,170 & 0 & 6,170 \\
\hline South Dakota..................... & 72.0 & 5.56 & 211 & 47.9 & 28.5 & 24.4 & 0 & 8.65 & 0 & 2.39 & 0 & 400 & 0 & 400 \\
\hline Tennessee ......................... & 850 & 42.8 & 63.8 & 23.4 & 56.9 & 734 & 0 & 31.4 & 0 & 4,620 & 0 & 6,420 & 0 & 6,420 \\
\hline 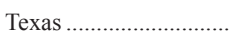 & 2,890 & 137 & 5,490 & 276 & 23.2 & 323 & 601 & 131 & 1,000 & 9,640 & 757 & 18,900 & 2,390 & 21,300 \\
\hline 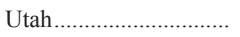 & 627 & 10.4 & 3,030 & 15.9 & 83.1 & 54.2 & 79.0 & 3.47 & 258 & 61.0 & 8.46 & 3,880 & 350 & 4,230 \\
\hline 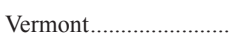 & 42.7 & 11.0 & 3.11 & 5.87 & 12.0 & 11.0 & 0 & 4.56 & 0 & 0.80 & 0 & 90.9 & 0 & 90.9 \\
\hline 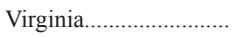 & 697 & 125 & 51.7 & 27.0 & 113 & 370 & 5.15 & 24.9 & 0 & 2,910 & 2,400 & 4,310 & 2,420 & 6,730 \\
\hline 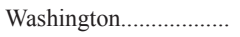 & 867 & 110 & 2,520 & 29.7 & 245 & 412 & 0 & 17.0 & 0 & 52.2 & 0 & 4,260 & 0 & 4,260 \\
\hline West Virginia................... & 185 & 31.3 & 4.15 & 5.08 & 39.3 & 424 & 3.05 & 53.3 & 1.52 & 1,570 & 0 & 2,320 & 4.57 & 2,320 \\
\hline Wisconsin ......................... & 479 & 76.4 & 460 & 74.5 & 48.2 & 382 & 0 & 29.3 & 0 & 4,210 & 0 & 5,760 & 0 & 5,760 \\
\hline 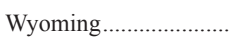 & 101 & 8.93 & 7,790 & 16.2 & 28.8 & 8.04 & 0 & 44.5 & 96.8 & 51.8 & 0 & 8,050 & 96.8 & 8,140 \\
\hline Puerto Rico....................... & 576 & 0.52 & 75.7 & 5.83 & 0.63 & 3.67 & 0 & 2.02 & 0 & 4.65 & 1,700 & 669 & 1,700 & 2,370 \\
\hline U.S. Virgin Islands ...... & 4.27 & 3.78 & 0 & 0.02 & 0 & 0.52 & 0 & 0 & 0.02 & 0.14 & 96.5 & 5.37 & 99.9 & 105 \\
\hline TOTAL & 39,000 & 3,260 & 118,000 & 2,000 & 7,550 & 14,000 & 786 & 1,880 & 2,120 & 95,100 & 37,800 & 281,000 & 41,000 & 322,000 \\
\hline
\end{tabular}


Table 2B. Total water withdrawals by water-use category, 2015, in thousand acre-feet per year.

[Values may not sum to totals because of independent rounding. Values for public supply and aquaculture include fresh and saline-water withdrawals]

\begin{tabular}{|c|c|c|c|c|c|c|c|c|c|c|c|c|c|c|}
\hline \multirow[t]{2}{*}{ State } & \multirow{2}{*}{$\begin{array}{c}\text { Public } \\
\text { supply }\end{array}$} & \multirow{2}{*}{$\begin{array}{c}\text { Domes- } \\
\text { tic }\end{array}$} & \multirow{2}{*}{$\begin{array}{c}\begin{array}{c}\text { Irriga- } \\
\text { tion }\end{array} \\
\text { Fresh }\end{array}$} & \multirow{2}{*}{$\begin{array}{l}\begin{array}{l}\text { Live- } \\
\text { stock }\end{array} \\
\text { Fresh }\end{array}$} & \multirow{2}{*}{$\begin{array}{c}\begin{array}{c}\text { Aqua- } \\
\text { culture }\end{array} \\
\text { Total }\end{array}$} & \multicolumn{2}{|c|}{ Industrial } & \multicolumn{2}{|c|}{ Mining } & \multicolumn{2}{|c|}{$\begin{array}{c}\text { Thermoelectric } \\
\text { power }\end{array}$} & \multicolumn{3}{|c|}{ Total } \\
\hline & & & & & & Fresh & Saline & Fresh & Saline & Fresh & Saline & Fresh & Saline & Total \\
\hline Alabama ............................ & 854 & 41.1 & 250 & 29.4 & 55.4 & 554 & 0 & 33.9 & 0 & 7,430 & 0 & 9,250 & 0 & 9,250 \\
\hline 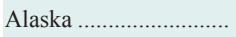 & 111 & 12.8 & 1.70 & 0.15 & 459 & 9.36 & 2.05 & 40.8 & 146 & 74.8 & 0 & 710 & 148 & 858 \\
\hline 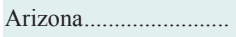 & 1,340 & 26.9 & 5,080 & 43.6 & 38.7 & 6.86 & 0 & 76.6 & 0 & 93.6 & 0 & 6,700 & 0 & 6,700 \\
\hline 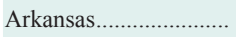 & 407 & 14.4 & 13,000 & 38.2 & 282 & 176 & 0 & 3.44 & 0 & 1,620 & 0 & 15,500 & 0 & 15,500 \\
\hline California ........................ & 5,770 & 142 & 21,300 & 205 & 815 & 447 & 0 & 51.3 & 305 & 40.8 & 3,180 & 28,700 & 3,550 & 32,200 \\
\hline Colorado.......................... & 946 & 39.6 & 10,100 & 37.3 & 292 & 94.2 & 0 & 8.63 & 27.1 & 41.7 & 0 & 11,500 & 27.1 & 11,600 \\
\hline Connecticut .................... & 269 & 34.6 & 12.7 & 1.29 & 28.2 & 203 & 46.7 & 4.76 & 0 & 142 & 2,770 & 691 & 2,820 & 3,510 \\
\hline 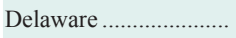 & 96.8 & 16.3 & 127 & 1.50 & 2.22 & 338 & 0 & 0.73 & 0 & 16.1 & 287 & 598 & 287 & 886 \\
\hline District of Columbia ... & 0 & 0 & 0.06 & 0 & 0 & 0 & 0 & 0 & 0 & 0 & 0 & 0.06 & 0 & 0.06 \\
\hline 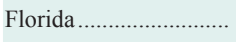 & 2,670 & 198 & 2,740 & 29.2 & 16.8 & 275 & 1.21 & 146 & 0 & 487 & 10,600 & 6,370 & 10,800 & 17,100 \\
\hline 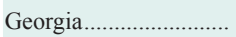 & 1,200 & 117 & 827 & 50.3 & 95.4 & 533 & 0 & 22.2 & 0 & 831 & 114 & 3,680 & 114 & 3,790 \\
\hline 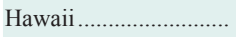 & 299 & 9.08 & 432 & 1.80 & 20.9 & 0.27 & 0 & 1.03 & 0 & 1.66 & 423 & 766 & 423 & 1,190 \\
\hline 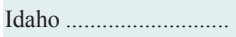 & 309 & 78.6 & 17,100 & 56.9 & 2,200 & 64.6 & 0 & 25.9 & 0 & 2.01 & 0 & 19,900 & 0 & 19,900 \\
\hline 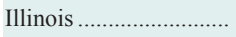 & 1,650 & 103 & 262 & 40.6 & 12.0 & 483 & 0 & 61.7 & 23.5 & 9,120 & 0 & 11,700 & 23.5 & 11,800 \\
\hline 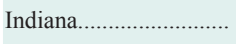 & 704 & 143 & 149 & 43.9 & 16.2 & 2,560 & 0 & 141 & 0 & 4,280 & 0 & 8,050 & 0 & 8,050 \\
\hline 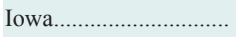 & 438 & 35.8 & 39.2 & 185 & 22.1 & 322 & 0 & 84.7 & 0 & 1,890 & 0 & 3,010 & 0 & 3,010 \\
\hline 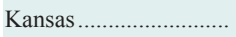 & 394 & 19.8 & 3,000 & 117 & 7.16 & 42.7 & 0 & 6.71 & 0 & 916 & 0 & 4,500 & 6.93 & 4,510 \\
\hline Kentucky ......................... & 620 & 24.8 & 44.3 & 45.7 & 54.3 & 252 & 0 & 45.5 & 0 & 2,090 & 0 & 3,180 & 0 & 3,180 \\
\hline 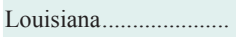 & 795 & 44.1 & 1,180 & 7.12 & 553 & 2,400 & 0 & 7.00 & 0 & 4,520 & 293 & 9,510 & 293 & 9,800 \\
\hline 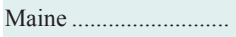 & 95.2 & 35.5 & 21.2 & 2.30 & 60.5 & 204 & 48.1 & 7.10 & 0 & 5.94 & 90.4 & 431 & 139 & 570 \\
\hline 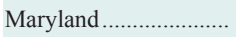 & 840 & 128 & 71.8 & 8.97 & 8.95 & 55.4 & 1.54 & 18.8 & 0 & 247 & 5,940 & 1,380 & 5,940 & 7,320 \\
\hline Massachusetts ............... & 726 & 39.4 & 155 & 1.15 & 11.1 & 31.2 & 0 & 10.8 & 0 & 56.2 & 546 & 1,030 & 547 & 1,580 \\
\hline 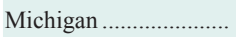 & 1,160 & 210 & 372 & 26.6 & 82.8 & 581 & 0 & 96.0 & 0.65 & 8,750 & 0 & 11,300 & 0.65 & 11,300 \\
\hline Minnesota........................ & 578 & 92.2 & 310 & 66.0 & 18.5 & 290 & 0 & 10.3 & 0 & 2,260 & 0 & 3,620 & 0 & 3,620 \\
\hline Mississippi ...................... & 449 & 54.0 & 1,980 & 19.2 & 142 & 204 & 0 & 10.6 & 7.38 & 132 & 9.44 & 2,990 & 16.8 & 3,010 \\
\hline 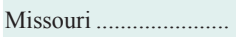 & 894 & 64.4 & 1,540 & 71.4 & 184 & 95.5 & 0 & 33.1 & 0 & 6,560 & 0 & 9,450 & 0 & 9,450 \\
\hline Montana ......................... & 172 & 26.6 & 10,600 & 47.3 & 19.2 & 10.8 & 0 & 24.2 & 18.3 & 84.8 & 0 & 11,000 & 18.3 & 11,000 \\
\hline Nebraska .......................... & 308 & 21.3 & 6,830 & 123 & 29.7 & 49.6 & 0 & 10.8 & 7.19 & 3,270 & 0 & 10,600 & 7.19 & 10,600 \\
\hline Nevada ........................... & 596 & 40.1 & 2,320 & 5.54 & 38.2 & 6.40 & 0 & 219 & 12.6 & 9.79 & 79.5 & 3,230 & 92.2 & 3,320 \\
\hline New Hampshire ........... & 107 & 33.3 & 5.83 & 0.94 & 19.2 & 14.2 & 0 & 6.87 & 0 & 83.9 & 777 & 271 & 777 & 1,050 \\
\hline New Jersey ...................... & 1,320 & 102 & 105 & 0.99 & 11.0 & 106 & 0 & 65.3 & 0 & 404 & 3,840 & 2,110 & 3,840 & 5,950 \\
\hline New Mexico.................... & 293 & 27.6 & 2,660 & 35.9 & 27.0 & 3.81 & 0 & 63.7 & 100 & 37.5 & 0 & 3,150 & 100 & 3,250 \\
\hline New York ….................... & 2,720 & 210 & 60.0 & 28.8 & 67.7 & 350 & 9.45 & 45.1 & 1.06 & 2,480 & 6,140 & 5,960 & 6,150 & 12,100 \\
\hline North Carolina .............. & 1,050 & 189 & 364 & 74.6 & 1,120 & 217 & 0 & 42.9 & 0 & 6,930 & 1,520 & 10,000 & 1,520 & 11,500 \\
\hline North Dakota.................... & 94.4 & 4.14 & 261 & 23.3 & 5.96 & 22.0 & 0 & 34.4 & 16.9 & 1,100 & 0 & 1,550 & 16.9 & 1,560 \\
\hline Ohio............................... & 1,460 & 156 & 61.6 & 27.6 & 44.1 & 390 & 0 & 145 & 0 & 5,030 & 0 & 7,310 & 0 & 7,310 \\
\hline 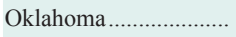 & 685 & 34.0 & 1,040 & 79.2 & 3.69 & 58.3 & 0 & 42.0 & 173 & 80.4 & 0 & 2,030 & 173 & 2,200 \\
\hline 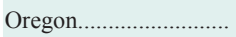 & 636 & 82.8 & 5,780 & 18.2 & 710 & 117 & 0 & 12.7 & 0 & 12.7 & 0 & 7,370 & 0 & 7,370 \\
\hline Pennsylvania .................... & 1,560 & 233 & 38.5 & 44.3 & 108 & 723 & 0 & 42.7 & 6.28 & 4,010 & 0 & 6,760 & 6.28 & 6,770 \\
\hline Rhode Island .................... & 109 & 7.36 & 4.76 & 0.13 & 7.63 & 2.30 & 0.03 & 3.27 & 0 & 1.49 & 249 & 136 & 249 & 385 \\
\hline South Carolina .............. & 710 & 133 & 141 & 11.1 & 7.50 & 321 & 0 & 11.3 & 0 & 5,580 & 0 & 6,920 & 0 & 6,920 \\
\hline South Dakota.................... & 80.7 & 6.23 & 236 & 53.6 & 31.9 & 27.4 & 0 & 9.70 & 0 & 2.68 & 0 & 448 & 0 & 448 \\
\hline Tennessee ........................ & 952 & 48.0 & 71.6 & 26.3 & 63.8 & 822 & 0 & 35.2 & 0 & 5,180 & 0 & 7,200 & 0 & 7,200 \\
\hline 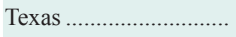 & 3,230 & 153 & 6,150 & 309 & 26.0 & 362 & 674 & 147 & 1,130 & 10,800 & 849 & 21,200 & 2,670 & 23,800 \\
\hline 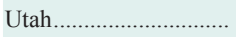 & 702 & 11.6 & 3,390 & 17.8 & 93.1 & 60.7 & 88.5 & 3.89 & 289 & 68.4 & 9.48 & 4,340 & 392 & 4,740 \\
\hline 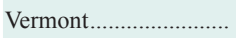 & 47.8 & 12.3 & 3.49 & 6.58 & 13.5 & 12.3 & 0 & 5.11 & 0 & 0.90 & 0 & 102 & 0 & 102 \\
\hline 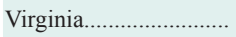 & 782 & 140 & 57.9 & 30.3 & 127 & 415 & 5.77 & 27.9 & 0 & 3,270 & 2,690 & 4,830 & 2,710 & 7,540 \\
\hline 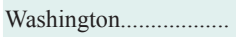 & 971 & 123 & 2,830 & 33.3 & 275 & 462 & 0 & 19.1 & 0 & 58.5 & 0 & 4,770 & 0 & 4,770 \\
\hline West Virginia.................... & 207 & 35.1 & 4.65 & 5.69 & 44.1 & 476 & 3.42 & 59.8 & 1.70 & 1,760 & 0 & 2,600 & 5.12 & 2,600 \\
\hline Wisconsin ........................ & 537 & 85.7 & 515 & 83.5 & 54.0 & 429 & 0 & 32.8 & 0 & 4,710 & 0 & 6,450 & 0 & 6,450 \\
\hline 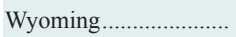 & 114 & 10.0 & 8,730 & 18.1 & 32.3 & 9.01 & 0 & 49.8 & 108 & 58.1 & 0 & 9,020 & 108 & 9,130 \\
\hline Puerto Rico....................... & 646 & 0.58 & 84.8 & 6.54 & 0.71 & 4.11 & 0 & 2.26 & 0 & 5.21 & 1,910 & 750 & 1,910 & 2,660 \\
\hline U.S. Virgin Islands ...... & 4.79 & 4.24 & 0 & 0.02 & 0 & 0.58 & 0 & 0 & 0.02 & 0.16 & 108 & 6.02 & 112 & 118 \\
\hline TOTAL & 43,700 & 3,650 & 132,000 & 2,240 & 8,460 & 15,700 & 881 & 2,110 & 2,370 & 107,000 & 42,400 & 315,000 & 45,900 & 361,000 \\
\hline
\end{tabular}



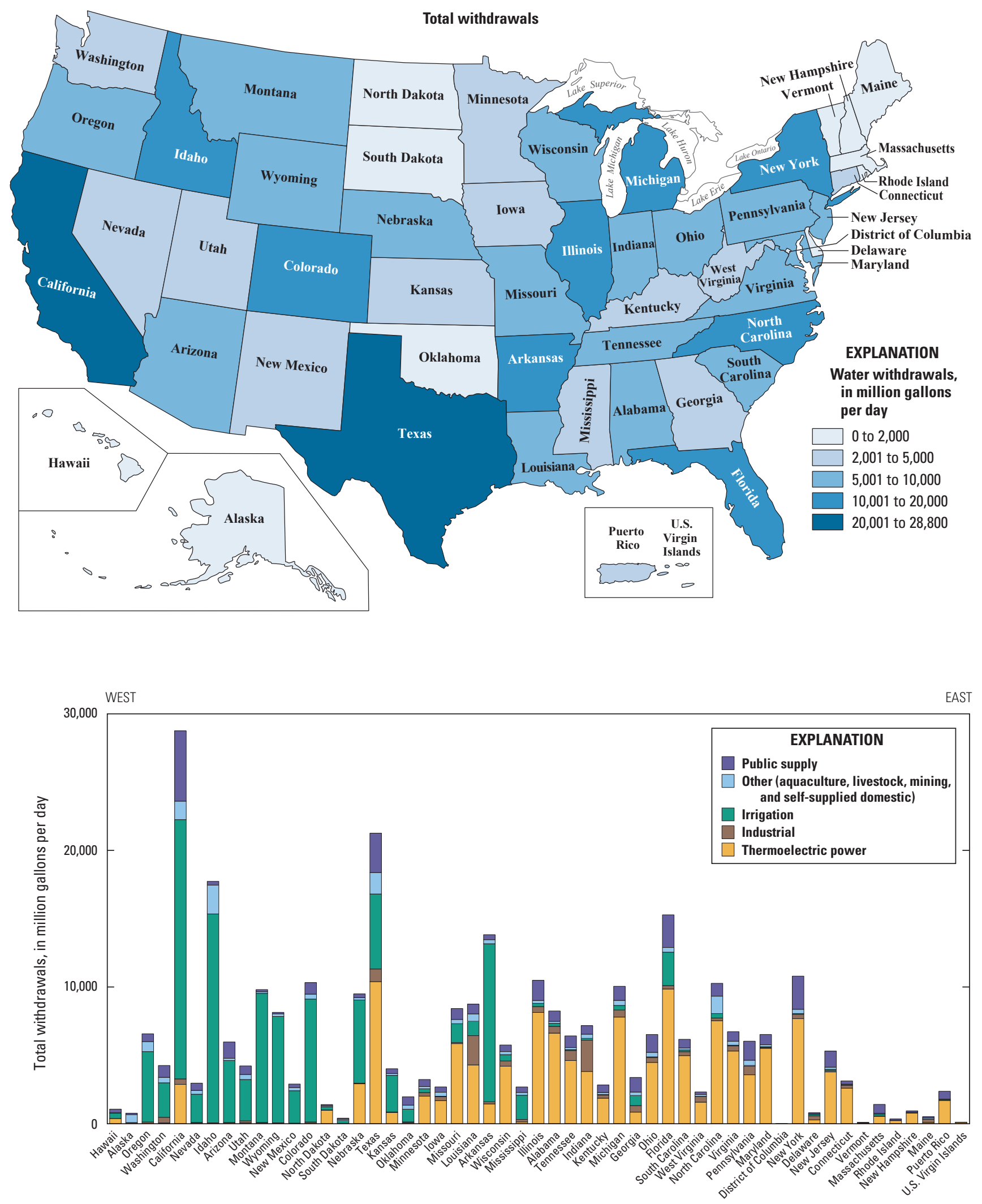

Figure 2. Total water withdrawals by State, and bar chart showing categories by State from west to east, 2015. 

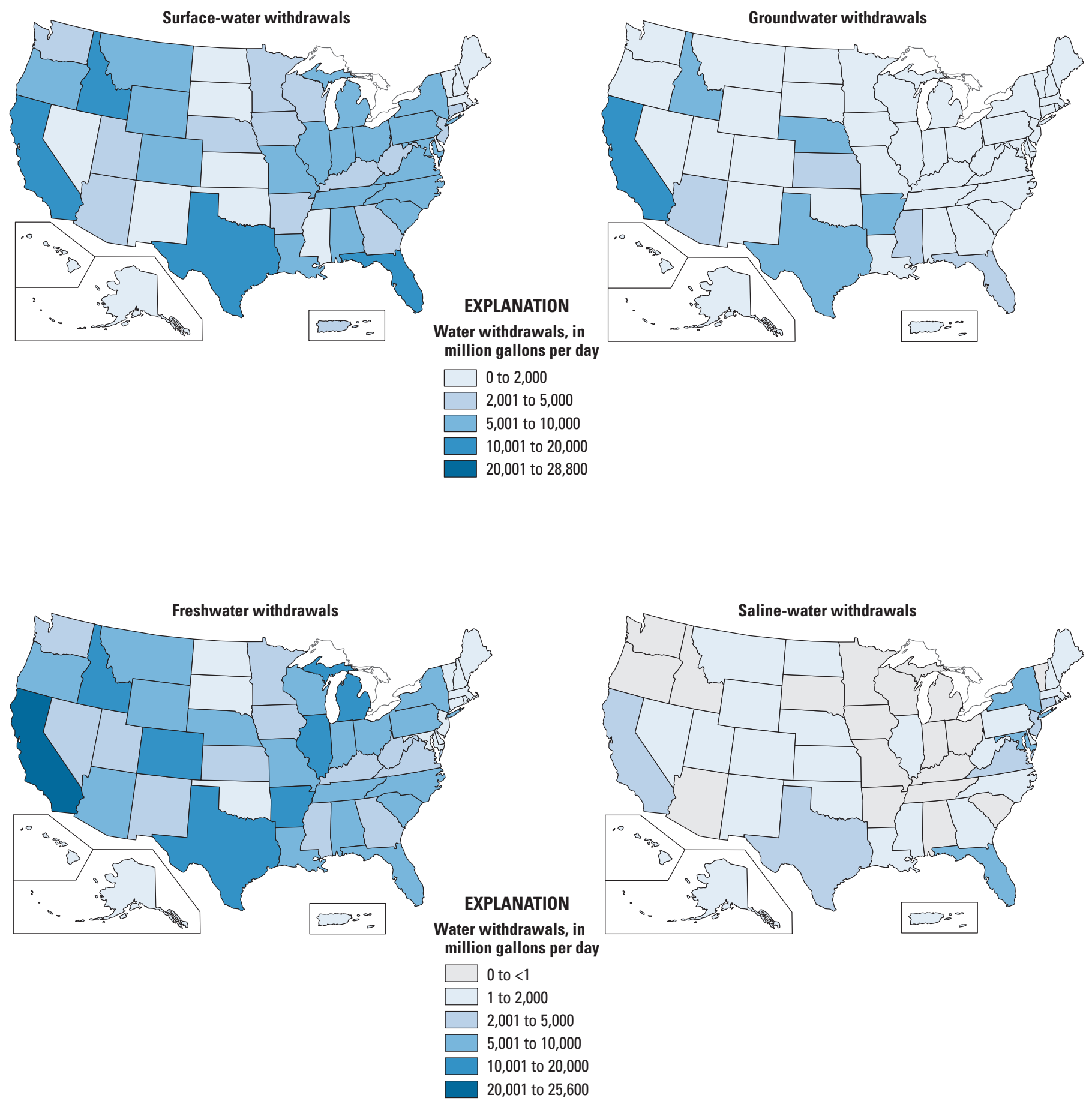

Figure 3. Surface-water and groundwater withdrawals, and freshwater and saline-water withdrawals, 2015. 
Table 3A. Surface-water withdrawals by water-use category, 2015, in million gallons per day.

[Values may not sum to totals because of independent rounding. Values for public supply and aquaculture include fresh and saline-water withdrawals]

\begin{tabular}{|c|c|c|c|c|c|c|c|c|c|c|c|c|c|c|}
\hline \multirow[t]{2}{*}{ State } & \multirow{2}{*}{$\begin{array}{c}\text { Public } \\
\text { supply } \\
\text { Total }\end{array}$} & \multirow{2}{*}{$\begin{array}{c}\text { Domes- } \\
\text { tic }\end{array}$} & \multirow{2}{*}{$\begin{array}{c}\begin{array}{c}\text { Irriga- } \\
\text { tion }\end{array} \\
\text { Fresh }\end{array}$} & \multirow{2}{*}{$\begin{array}{l}\begin{array}{c}\text { Live- } \\
\text { stock }\end{array} \\
\text { Fresh }\end{array}$} & \multirow{2}{*}{$\begin{array}{c}\begin{array}{c}\text { Aqua- } \\
\text { culture }\end{array} \\
\text { Total }\end{array}$} & \multicolumn{2}{|c|}{ Industrial } & \multicolumn{2}{|c|}{ Mining } & \multicolumn{2}{|c|}{$\begin{array}{c}\text { Thermoelectric } \\
\text { power }\end{array}$} & \multicolumn{3}{|c|}{ Total } \\
\hline & & & & & & Fresh & Saline & Fresh & Saline & Fresh & Saline & Fresh & Saline & Total \\
\hline Alabama ............................ & 490 & 0 & 124 & 14.7 & 22.2 & 461 & 0 & 8.45 & 0 & 6,630 & 0 & 7,750 & 0 & 7,750 \\
\hline 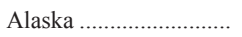 & 61.4 & 1.59 & 0.02 & 0.09 & 240 & 1.65 & 1.83 & 36.4 & 41.4 & 66.1 & 0 & 408 & 43.2 & 451 \\
\hline 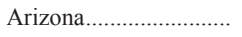 & 626 & 0 & 2,560 & 0 & 10.7 & 0 & 0 & 0 & 0 & 25.8 & 0 & 3,220 & 0 & 3,220 \\
\hline 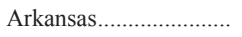 & 269 & 0 & 2,290 & 20.5 & 98.9 & 127 & 0 & 2.93 & 0 & 1,440 & 0 & 4,250 & 0 & 4,250 \\
\hline California ......................... & 2,780 & 18.5 & 5,130 & 101 & 480 & 1.13 & 0 & 15.6 & 0.01 & 16.7 & 2,800 & 8,540 & 2,800 & 11,300 \\
\hline 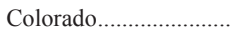 & 735 & 0 & 7,690 & 10.6 & 244 & 79.9 & 0 & 2.46 & 0 & 34.1 & 0 & 8,800 & 0 & 8,800 \\
\hline Connecticut .................... & 197 & 0 & 8.29 & 0 & 16.0 & 141 & 41.6 & 3.43 & 0 & 126 & 2,470 & 489 & 2,510 & 3,000 \\
\hline Delaware ........................ & 40.6 & 0 & 17.5 & 0 & 0 & 291 & 0 & 0.31 & 0 & 14.3 & 256 & 364 & 256 & 620 \\
\hline District of Columbia ... & 0 & 0 & 0.05 & 0 & 0 & 0 & 0 & 0 & 0 & 0 & 0 & 0.05 & 0 & 0.05 \\
\hline 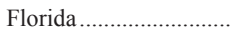 & 307 & 0 & 1,290 & 0.35 & 2.10 & 63.6 & 1.08 & 40.7 & 0 & 406 & 9,400 & 2,110 & 9,400 & 11,500 \\
\hline Georgia..... & 839 & 0 & 175 & 17.4 & 70.6 & 283 & 0 & 3.89 & 0 & 738 & 102 & 2,130 & 102 & 2,230 \\
\hline 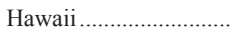 & 14.6 & 7.44 & 311 & 1.10 & 10.0 & 0 & 0 & 0.03 & 0 & 0 & 357 & 344 & 357 & 702 \\
\hline 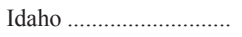 & 29.2 & 0 & 10,400 & 9.57 & 1,920 & 12.7 & 0 & 21.8 & 0 & 0 & 0 & 12,400 & 0 & 12,400 \\
\hline 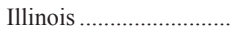 & 1,110 & 0 & 14.6 & 0 & 5.47 & 299 & 0 & 40.4 & 0 & 8,130 & 0 & 9,600 & 0 & 9,600 \\
\hline Indiana........................... & 288 & 0 & 40.0 & 13.0 & 8.00 & 2,210 & 0 & 121 & 0 & 3,800 & 0 & 6,480 & 0 & 6,480 \\
\hline Iowa.................................... & 76.8 & 0 & 2.84 & 41.0 & 12.2 & 195 & 0 & 74.4 & 0 & 1,660 & 0 & 2,060 & 0 & 2,060 \\
\hline 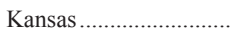 & 214 & 0 & 122 & 20.4 & 3.23 & 7.63 & 0 & 0.55 & 0 & 809 & 0 & 1,180 & 0 & 1,180 \\
\hline 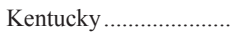 & 465 & 7.63 & 36.7 & 38.7 & 47.8 & 148 & 0 & 22.9 & 0 & 1,860 & 0 & 2,630 & 0 & 2,630 \\
\hline Louisiana .......................... & 355 & 0 & 333 & 3.19 & 156 & 1,890 & 0 & 4.59 & 0 & 4,000 & 261 & 6,740 & 261 & 7,000 \\
\hline 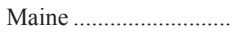 & 57.5 & 0 & 13.8 & 0.51 & 43.1 & 176 & 42.9 & 4.88 & 0 & 4.38 & 80.7 & 300 & 124 & 424 \\
\hline 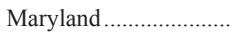 & 656 & 0 & 15.8 & 2.15 & 2.07 & 38.6 & 1.37 & 3.09 & 0 & 218 & 5,300 & 935 & 5,300 & 6,230 \\
\hline Massachusetts .............. & 449 & 0 & 24.1 & 0.39 & 1.80 & 12.7 & 0 & 4.09 & 0 & 49.0 & 487 & 540 & 488 & 1,030 \\
\hline Michigan ........................... & 821 & 0 & 70.6 & 2.33 & 72.8 & 453 & 0 & 69.7 & 0 & 7,800 & 0 & 9,290 & 0 & 9,290 \\
\hline Minnesota......................... & 179 & 0 & 33.9 & 0 & 14.8 & 210 & 0 & 5.57 & 0 & 2,010 & 0 & 2,450 & 0 & 2,450 \\
\hline Mississippi ...................... & 53.4 & 0 & 130 & 10.3 & 39.3 & 109 & 0 & 1.24 & 0 & 84.0 & 2.07 & 427 & 2.07 & 429 \\
\hline 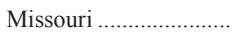 & 516 & 0 & 75.3 & 47.6 & 157 & 42.8 & 0 & 8.47 & 0 & 5,840 & 0 & 6,690 & 0 & 6,690 \\
\hline Montana .......................... & 69.9 & 1.12 & 9,390 & 29.9 & 13.6 & 4.45 & 0 & 20.5 & 0 & 74.9 & 0 & 9,610 & 0 & 9,610 \\
\hline Nebraska ......................... & 57.2 & 0 & 674 & 20.4 & 16.0 & 2.32 & 0 & 9.54 & 0 & 2,900 & 0 & 3,680 & 0 & 3,680 \\
\hline Nevada ........................... & 390 & 0 & 1,090 & 0 & 22.4 & 4.69 & 0 & 8.13 & 0 & 1.69 & 0 & 1,520 & 0 & 1,520 \\
\hline New Hampshire ........... & 60.1 & 0 & 4.11 & 0.21 & 10.7 & 8.41 & 0 & 4.37 & 0 & 73.7 & 693 & 162 & 693 & 855 \\
\hline New Jersey ..................... & 797 & 0 & 38.8 & 0 & 0 & 64.1 & 0 & 56.8 & 0 & 358 & 3,430 & 1,310 & 3,430 & 4,740 \\
\hline New Mexico.................. & 78.3 & 0 & 1,320 & 2.25 & 5.45 & 0 & 0 & 17.4 & 0 & 27.3 & 0 & 1,460 & 0 & 1,460 \\
\hline New York ....................... & 1,810 & 0 & 35.7 & 8.89 & 49.5 & 283 & 8.43 & 34.6 & 0 & 2,200 & 5,470 & 4,420 & 5,480 & 9,910 \\
\hline North Carolina .............. & 780 & 0 & 241 & 14.6 & 990 & 178 & 0 & 8.26 & 0 & 6,180 & 1,360 & 8,400 & 1,360 & 9,750 \\
\hline North Dakota................ & 48.8 & 0 & 130 & 8.30 & 5.32 & 13.5 & 0 & 4.50 & 0 & 983 & 0 & 1,190 & 0 & 1,190 \\
\hline Ohio................................ & 857 & 2.72 & 37.1 & 16.8 & 26.0 & 210 & 0 & 37.6 & 0 & 4,470 & 0 & 5,660 & 0 & 5,660 \\
\hline 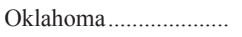 & 509 & 0 & 144 & 43.3 & 3.23 & 44.7 & 0 & 33.2 & 0 & 70.2 & 0 & 848 & 0 & 848 \\
\hline 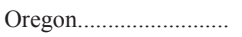 & 420 & 7.54 & 3,940 & 13.2 & 601 & 101 & 0 & 2.85 & 0 & 9.79 & 0 & 5,100 & 0 & 5,100 \\
\hline Pennsylvania ................... & 1,160 & 0 & 16.8 & 3.17 & 47.2 & 599 & 0 & 5.21 & 0 & 3,570 & 0 & 5,410 & 0 & 5,410 \\
\hline Rhode Island ................... & 83.7 & 0 & 0.47 & 0.01 & 0.09 & 1.51 & 0.03 & 1.74 & 0 & 1.33 & 222 & 88.8 & 222 & 311 \\
\hline South Carolina .............. & 518 & 0 & 38.3 & 5.51 & 6.08 & 260 & 0 & 1.79 & 0 & 4,980 & 0 & 5,810 & 0 & 5,810 \\
\hline South Dakota..................... & 24.0 & 0 & 71.2 & 28.6 & 24.9 & 6.04 & 0 & 5.06 & 0 & 2.39 & 0 & 162 & 0 & 162 \\
\hline Tennessee ....................... & 594 & 0 & 27.4 & 11.5 & 45.2 & 682 & 0 & 14.2 & 0 & 4,620 & 0 & 5,990 & 0 & 5,990 \\
\hline 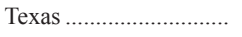 & 1,710 & 0 & 1,010 & 137 & 11.6 & 223 & 598 & 15.9 & 0.01 & 9,600 & 757 & 12,700 & 1,360 & 14,100 \\
\hline 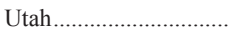 & 267 & 0 & 2,490 & 8.44 & 0 & 16.8 & 42.4 & 1.80 & 214 & 38.3 & 0 & 2,820 & 257 & 3,080 \\
\hline 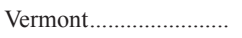 & 28.8 & 0 & 2.09 & 1.46 & 7.84 & 9.46 & 0 & 4.33 & 0 & 0.26 & 0 & 54.2 & 0 & 54.2 \\
\hline 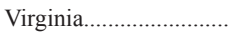 & 614 & 0 & 43.8 & 20.5 & 113 & 304 & 5.15 & 18.7 & 0 & 2,910 & 2,400 & 4,030 & 2,400 & 6,430 \\
\hline Washington........................ & 345 & 0 & 1,800 & 8.82 & 194 & 329 & 0 & 3.61 & 0 & 44.4 & 0 & 2,730 & 0 & 2,730 \\
\hline West Virginia.................... & 147 & 0 & 3.75 & 3.38 & 31.2 & 399 & 0 & 29.5 & 0 & 1,570 & 0 & 2,190 & 0 & 2,190 \\
\hline 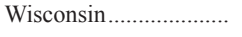 & 214 & 0 & 173 & 7.44 & 22.6 & 336 & 0 & 28.8 & 0 & 4,200 & 0 & 4,980 & 0 & 4,980 \\
\hline 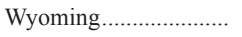 & 46.8 & 0 & 7,250 & 10.0 & 23.9 & 2.21 & 0 & 11.4 & 0 & 50.5 & 0 & 7,400 & 0 & 7,400 \\
\hline Puerto Rico...................... & 510 & 0 & 34.1 & 1.60 & 0.63 & 0 & 0 & 0.18 & 0 & 2.83 & 1,700 & 549 & 1,700 & 2,250 \\
\hline U.S. Virgin Islands ...... & 3.36 & 2.55 & 0 & 0.01 & 0 & 0 & 0 & 0 & 0.02 & 0.14 & 96.5 & 2.70 & 99.9 & 103 \\
\hline TOTAL & 23,800 & 49.1 & 60,900 & 760 & 5,950 & 11,300 & 743 & 877 & 256 & 94,700 & 37,600 & 198,000 & 38,600 & 237,000 \\
\hline
\end{tabular}


Table 3B. Surface-water withdrawals by water-use category, 2015, in thousand acre-feet per year.

[Values may not sum to totals because of independent rounding. Values for public supply and aquaculture include fresh and saline-water withdrawals]

\begin{tabular}{|c|c|c|c|c|c|c|c|c|c|c|c|c|c|c|}
\hline \multirow[t]{2}{*}{ State } & \multirow{2}{*}{$\begin{array}{c}\text { Public } \\
\text { supply } \\
\text { Total } \\
\end{array}$} & \multirow{2}{*}{$\begin{array}{c}\text { Domes- } \\
\text { tic }\end{array}$} & \multirow{2}{*}{$\begin{array}{c}\text { Irriga- } \\
\text { tion }\end{array}$} & \multirow{2}{*}{$\begin{array}{l}\begin{array}{l}\text { Live- } \\
\text { stock }\end{array} \\
\text { Fresh }\end{array}$} & \multirow{2}{*}{$\begin{array}{c}\begin{array}{c}\text { Aqua- } \\
\text { culture }\end{array} \\
\text { Total }\end{array}$} & \multicolumn{2}{|c|}{ Industrial } & \multicolumn{2}{|c|}{ Mining } & \multicolumn{2}{|c|}{$\begin{array}{c}\text { Thermoelectric } \\
\text { power }\end{array}$} & \multicolumn{3}{|c|}{ Total } \\
\hline & & & & & & Fresh & Saline & Fresh & Saline & Fresh & Saline & Fresh & Saline & Total \\
\hline Alabama ........................... & 549 & 0 & 139 & 16.4 & 24.8 & 517 & 0 & 9.47 & 0 & 7,430 & 0 & 8,680 & 0 & 8,680 \\
\hline Alaska ............................. & 68.8 & 1.78 & 0.02 & 0.10 & 269 & 1.85 & 2.05 & 40.8 & 46.4 & 74.1 & 0 & 457 & 48.4 & 505 \\
\hline Arizona........................... & 702 & 0 & 2,870 & 0 & 12.0 & 0 & 0 & 0 & 0 & 28.9 & 0 & 3,610 & 0 & 3,610 \\
\hline 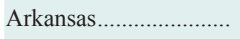 & 301 & 0 & 2,560 & 23.0 & 111 & 143 & 0 & 3.28 & 0 & 1,620 & 0 & 4,760 & 0 & 4,760 \\
\hline California ........................ & 3,120 & 20.8 & 5,750 & 113 & 538 & 1.27 & 0 & 17.4 & 0.01 & 18.8 & 3,140 & 9,580 & 3,140 & 12,700 \\
\hline Colorado........................... & 824 & 0 & 8,620 & 11.8 & 274 & 89.5 & 0 & 2.76 & 0 & 38.3 & 0 & 9,860 & 0 & 9,860 \\
\hline Connecticut ..................... & 221 & 0 & 9.29 & 0 & 18.0 & 158 & 46.7 & 3.85 & 0 & 142 & 2,770 & 548 & 2,820 & 3,360 \\
\hline Delaware .......................... & 45.5 & 0 & 19.6 & 0 & 0 & 326 & 0 & 0.35 & 0 & 16.0 & 287 & 408 & 287 & 695 \\
\hline District of Columbia ... & 0 & 0 & 0.06 & 0 & 0 & 0 & 0 & 0 & 0 & 0 & 0 & 0.06 & 0 & 0.06 \\
\hline 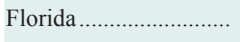 & 344 & 0 & 1,450 & 0.39 & 2.35 & 71.3 & 1.21 & 45.7 & 0 & 455 & 10,500 & 2,370 & 10,500 & 12,900 \\
\hline 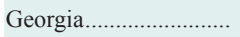 & 941 & 0 & 196 & 19.5 & 79.1 & 317 & 0 & 4.36 & 0 & 827 & 114 & 2,380 & 114 & 2,500 \\
\hline 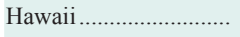 & 16.4 & 8.34 & 349 & 1.23 & 11.2 & 0 & 0 & 0.03 & 0 & 0 & 400 & 386 & 400 & 787 \\
\hline 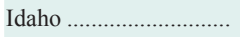 & 32.7 & 0 & 11,600 & 10.7 & 2,150 & 14.2 & 0 & 24.4 & 0 & 0 & 0 & 13,900 & 0 & 13,900 \\
\hline 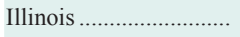 & 1,240 & 0 & 16.3 & 0 & 6.13 & 335 & 0 & 45.3 & 0 & 9,120 & 0 & 10,800 & 0 & 10,800 \\
\hline Indiana............................. & 323 & 0 & 44.8 & 14.5 & 8.97 & 2,470 & 0 & 135 & 0 & 4,260 & 0 & 7,260 & 0 & 7,260 \\
\hline Iowa.................................... & 86.1 & 0 & 3.18 & 46.0 & 13.7 & 218 & 0 & 83.4 & 0 & 1,860 & 0 & 2,310 & 0 & 2,310 \\
\hline Kansas .............................. & 240 & 0 & 136 & 22.9 & 3.62 & 8.55 & 0 & 0.62 & 0 & 907 & 0 & 1,320 & 0 & 1,320 \\
\hline Kentucky ........................ & 522 & 8.55 & 41.2 & 43.4 & 53.6 & 166 & 0 & 25.7 & 0 & 2,080 & 0 & 2,940 & 0 & 2,940 \\
\hline Louisiana......................... & 398 & 0 & 373 & 3.58 & 175 & 2,120 & 0 & 5.15 & 0 & 4,480 & 293 & 7,560 & 293 & 7,850 \\
\hline 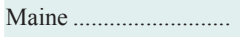 & 64.5 & 0 & 15.5 & 0.57 & 48.3 & 198 & 48.1 & 5.47 & 0 & 4.91 & 90.4 & 336 & 139 & 475 \\
\hline Maryland .......................... & 735 & 0 & 17.7 & 2.41 & 2.32 & 43.3 & 1.54 & 3.46 & 0 & 244 & 5,940 & 1,050 & 5,940 & 6,990 \\
\hline Massachusetts .............. & 503 & 0 & 27.0 & 0.44 & 2.02 & 14.2 & 0 & 4.58 & 0 & 55.0 & 546 & 605 & 547 & 1,150 \\
\hline Michigan .......................... & 920 & 0 & 79.2 & 2.61 & 81.6 & 508 & 0 & 78.1 & 0 & 8,740 & 0 & 10,400 & 0 & 10,400 \\
\hline 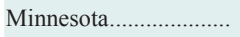 & 201 & 0 & 38.0 & 0 & 16.6 & 235 & 0 & 6.24 & 0 & 2,250 & 0 & 2,750 & 0 & 2,750 \\
\hline Mississippi ........................ & 59.8 & 0 & 145 & 11.5 & 44.1 & 122 & 0 & 1.39 & 0 & 94.2 & 2.32 & 479 & 2.32 & 481 \\
\hline Missouri .......................... & 578 & 0 & 84.4 & 53.3 & 176 & 48.0 & 0 & 9.49 & 0 & 6,550 & 0 & 7,500 & 0 & 7,500 \\
\hline Montana .......................... & 78.3 & 1.26 & 10,500 & 33.5 & 15.3 & 4.99 & 0 & 23.0 & 0 & 84.0 & 0 & 10,800 & 0 & 10,800 \\
\hline Nebraska ......................... & 64.2 & 0 & 755 & 22.9 & 17.9 & 2.60 & 0 & 10.7 & 0 & 3,250 & 0 & 4,130 & 0 & 4,130 \\
\hline Nevada ........................... & 437 & 0 & 1,230 & 0 & 25.1 & 5.26 & 0 & 9.11 & 0 & 1.89 & 0 & 1,700 & 0 & 1,700 \\
\hline New Hampshire ........... & 67.4 & 0 & 4.61 & 0.24 & 12.0 & 9.43 & 0 & 4.90 & 0 & 82.6 & 777 & 181 & 777 & 958 \\
\hline New Jersey ...................... & 893 & 0 & 43.5 & 0 & 0 & 71.8 & 0 & 63.6 & 0 & 402 & 3,840 & 1,470 & 3,840 & 5,310 \\
\hline New Mexico .................. & 87.8 & 0 & 1,490 & 2.52 & 6.11 & 0 & 0 & 19.6 & 0 & 30.6 & 0 & 1,630 & 0 & 1,630 \\
\hline New York ........................ & 2,030 & 0 & 40.0 & 9.97 & 55.4 & 317 & 9.45 & 38.8 & 0 & 2,470 & 6,140 & 4,960 & 6,150 & 11,100 \\
\hline North Carolina ............. & 875 & 0 & 270 & 16.3 & 1,110 & 200 & 0 & 9.26 & 0 & 6,930 & 1,520 & 9,410 & 1,520 & 10,900 \\
\hline North Dakota................... & 54.7 & 0 & 146 & 9.30 & 5.96 & 15.1 & 0 & 5.04 & 0 & 1,100 & 0 & 1,340 & 0 & 1,340 \\
\hline 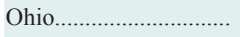 & 960 & 3.05 & 41.6 & 18.8 & 29.2 & 235 & 0 & 42.2 & 0 & 5,010 & 0 & 6,340 & 0 & 6,340 \\
\hline Oklahoma ......................... & 571 & 0 & 161 & 48.5 & 3.62 & 50.1 & 0 & 37.2 & 0 & 78.6 & 0 & 950 & 0 & 950 \\
\hline Oregon & 471 & 8.45 & 4,420 & 14.8 & 674 & 114 & 0 & 3.19 & 0 & 11.0 & 0 & 5,710 & 0 & 5,710 \\
\hline Pennsylvania ................... & 1,310 & 0 & 18.9 & 3.55 & 52.9 & 671 & 0 & 5.84 & 0 & 4,000 & 0 & 6,060 & 0 & 6,060 \\
\hline Rhode Island .................. & 93.8 & 0 & 0.53 & 0.01 & 0.10 & 1.69 & 0.03 & 1.95 & 0 & 1.49 & 249 & 99.6 & 249 & 348 \\
\hline South Carolina .............. & 581 & 0 & 43.0 & 6.18 & 6.82 & 292 & 0 & 2.01 & 0 & 5,580 & 0 & 6,510 & 0 & 6,510 \\
\hline South Dakota..................... & 26.9 & 0 & 79.8 & 32.0 & 27.9 & 6.77 & 0 & 5.67 & 0 & 2.68 & 0 & 182 & 0 & 182 \\
\hline Tennessee ........................ & 666 & 0 & 30.7 & 12.9 & 50.7 & 765 & 0 & 16.0 & 0 & 5,180 & 0 & 6,720 & 0 & 6,720 \\
\hline Texas ................................... & 1,920 & 0 & 1,140 & 154 & 13.0 & 250 & 670 & 17.8 & 0.01 & 10,800 & 849 & 14,300 & 1,520 & 15,800 \\
\hline 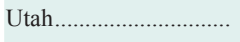 & 300 & 0 & 2,790 & 9.46 & 0 & 18.8 & 47.5 & 2.02 & 240 & 42.9 & 0 & 3,160 & 288 & 3,450 \\
\hline 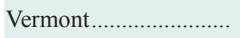 & 32.2 & 0 & 2.34 & 1.64 & 8.79 & 10.6 & 0 & 4.85 & 0 & 0.29 & 0 & 60.8 & 0 & 60.8 \\
\hline 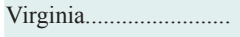 & 689 & 0 & 49.0 & 23.0 & 127 & 341 & 5.77 & 20.9 & 0 & 3,270 & 2,690 & 4,520 & 2,690 & 7,210 \\
\hline 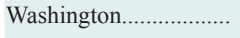 & 387 & 0 & 2,020 & 9.89 & 218 & 369 & 0 & 4.05 & 0 & 49.8 & 0 & 3,060 & 0 & 3,060 \\
\hline West Virginia.................... & 165 & 0 & 4.20 & 3.79 & 35.0 & 447 & 0 & 33.1 & 0 & 1,760 & 0 & 2,450 & 0 & 2,450 \\
\hline Wisconsin ............................ & 240 & 0 & 194 & 8.34 & 25.4 & 376 & 0 & 32.3 & 0 & 4,710 & 0 & 5,590 & 0 & 5,590 \\
\hline 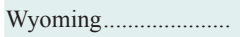 & 52.5 & 0 & 8,130 & 11.2 & 26.8 & 2.48 & 0 & 12.7 & 0 & 56.6 & 0 & 8,290 & 0 & 8,290 \\
\hline Puerto Rico...................... & 571 & 0 & 38.2 & 1.79 & 0.71 & 0 & 0 & 0.20 & 0 & 3.17 & 1,910 & 616 & 1,910 & 2,520 \\
\hline U.S. Virgin Islands ...... & 3.77 & 2.86 & 0 & 0.01 & 0 & 0 & 0 & 0 & 0.02 & 0.16 & 108 & 3.03 & 112 & 115 \\
\hline TOTAL & 26,700 & 55.0 & 68,300 & 852 & 6,670 & 12,700 & 833 & 983 & 287 & 106,000 & 42,200 & 222,000 & 43,300 & 266,000 \\
\hline
\end{tabular}


Table 4A. Groundwater withdrawals by water-use category, 2015, in million gallons per day.

[Values may not sum to totals because of independent rounding. Values for public supply and aquaculture include fresh and saline-water withdrawals]

\begin{tabular}{|c|c|c|c|c|c|c|c|c|c|c|c|c|c|c|}
\hline \multirow[t]{2}{*}{ State } & \multirow{2}{*}{$\begin{array}{c}\text { Public } \\
\text { supply } \\
\text { Total }\end{array}$} & \multirow{2}{*}{$\begin{array}{c}\text { Domes- } \\
\text { tic }\end{array}$} & \multirow{2}{*}{$\begin{array}{c}\begin{array}{c}\text { Irriga- } \\
\text { tion }\end{array} \\
\text { Fresh }\end{array}$} & \multirow{2}{*}{$\begin{array}{l}\begin{array}{l}\text { Live- } \\
\text { stock }\end{array} \\
\text { Fresh }\end{array}$} & \multirow{2}{*}{$\begin{array}{c}\begin{array}{c}\text { Aqua- } \\
\text { culture }\end{array} \\
\text { Total }\end{array}$} & \multicolumn{2}{|c|}{ Industrial } & \multicolumn{2}{|c|}{ Mining } & \multicolumn{2}{|c|}{$\begin{array}{c}\text { Thermoelectric } \\
\text { power }\end{array}$} & \multicolumn{3}{|c|}{ Total } \\
\hline & & & & & & Fresh & Saline & Fresh & Saline & Fresh & Saline & Fresh & Saline & Total \\
\hline Alabama ............................ & 272 & 36.7 & 98.8 & 11.5 & 27.2 & 32.7 & 0 & 21.8 & 0 & 0 & 0 & 501 & 0 & 501 \\
\hline 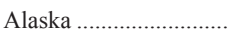 & 37.8 & 9.83 & 1.50 & 0.04 & 169 & 6.70 & 0 & 0 & 89.2 & 0.63 & 0 & 226 & 89.2 & 315 \\
\hline Arizona............................ & 569 & 24.0 & 1,970 & 38.9 & 23.8 & 6.12 & 0 & 68.3 & 0 & 57.7 & 0 & 2,760 & 0 & 2,760 \\
\hline 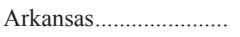 & 94.2 & 12.8 & 9,280 & 13.6 & 152 & 29.5 & 0 & 0.14 & 0 & 3.13 & 0 & 9,590 & 0 & 9,590 \\
\hline California ....................... & 2,370 & 108 & 13,900 & 82.0 & 247 & 397 & 0 & 30.2 & 272 & 19.6 & 35.1 & 17,100 & 359 & 17,400 \\
\hline 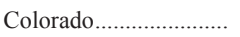 & 109 & 35.4 & 1,310 & 22.7 & 16.0 & 4.22 & 0 & 5.24 & 24.2 & 3.03 & 0 & 1,500 & 24.2 & 1,530 \\
\hline Connecticut .................... & 43.0 & 30.8 & 3.02 & 1.15 & 9.15 & 40.1 & 0 & 0.82 & 0 & 0 & 0 & 128 & 0 & 128 \\
\hline Delaware ......................... & 45.8 & 14.5 & 95.4 & 1.34 & 1.98 & 10.7 & 0 & 0.34 & 0 & 0.13 & 0 & 170 & 0 & 170 \\
\hline District of Columbia ... & 0 & 0 & 0 & 0 & 0 & 0 & 0 & 0 & 0 & 0 & 0 & 0 & 0 & 0 \\
\hline 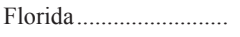 & 2,080 & 177 & 1,150 & 25.7 & 12.8 & 181 & 0 & 89.1 & 0 & 27.8 & 28.5 & 3,580 & 198 & 3,770 \\
\hline 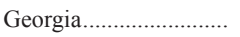 & 231 & 104 & 564 & 27.5 & 14.5 & 193 & 0 & 15.9 & 0 & 3.43 & 0 & 1,150 & 0 & 1,150 \\
\hline 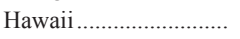 & 252 & 0.66 & 73.8 & 0.51 & 8.58 & 0.24 & 0 & 0.89 & 0 & 1.48 & 20.2 & 338 & 20.2 & 359 \\
\hline 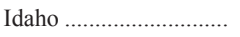 & 247 & 70.2 & 4,900 & 41.2 & 46.2 & 45.0 & 0 & 1.35 & 0 & 1.79 & 0 & 5,350 & 0 & 5,350 \\
\hline Illinois ............................ & 367 & 92.1 & 219 & 36.2 & 5.23 & 132 & 0 & 14.6 & 21.0 & 4.21 & 0 & 870 & 21.0 & 891 \\
\hline Indiana............................ & 339 & 127 & 93.1 & 26.2 & 6.46 & 79.9 & 0 & 5.52 & 0 & 20.8 & 0 & 699 & 0 & 699 \\
\hline Iowa................................ & 314 & 32.0 & 32.2 & 124 & 7.46 & 92.8 & 0 & 1.08 & 0 & 27.0 & 0 & 630 & 0 & 630 \\
\hline 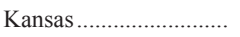 & 137 & 17.7 & 2,560 & 83.5 & 3.16 & 30.5 & 0 & 5.44 & 0 & 7.81 & 0 & 2,840 & 6.18 & 2,840 \\
\hline Kentucky ........................ & 87.4 & 14.5 & 2.84 & 2.07 & 0.58 & 77.3 & 0 & 17.6 & 0 & 4.96 & 0 & 207 & 0 & 207 \\
\hline Louisiana .......................... & 354 & 39.3 & 720 & 3.16 & 337 & 250 & 0 & 1.65 & 0 & 37.0 & 0 & 1,740 & 0 & 1,740 \\
\hline Maine …......................... & 27.5 & 31.6 & 5.06 & 1.54 & 10.9 & 5.83 & 0 & 1.45 & 0 & 0.92 & 0 & 84.8 & 0 & 84.8 \\
\hline Maryland .......................... & 93.9 & 114 & 48.3 & 5.85 & 5.91 & 10.8 & 0 & 13.7 & 0 & 2.30 & 0 & 295 & 0 & 295 \\
\hline Massachusetts .............. & 199 & 35.1 & 114 & 0.64 & 8.13 & 15.2 & 0 & 5.51 & 0 & 1.10 & 0 & 380 & 0 & 380 \\
\hline Michigan .......................... & 209 & 187 & 261 & 21.4 & 1.06 & 64.6 & 0 & 16.0 & 0.58 & 5.61 & 0 & 766 & 0.58 & 767 \\
\hline Minnesota........................ & 336 & 82.2 & 242 & 58.9 & 1.72 & 49.0 & 0 & 3.63 & 0 & 1.77 & 0 & 776 & 0 & 776 \\
\hline Mississippi …................... & 347 & 48.1 & 1,640 & 6.85 & 87.5 & 72.8 & 0 & 8.21 & 6.58 & 33.7 & 6.35 & 2,240 & 12.9 & 2,260 \\
\hline 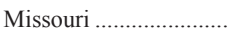 & 282 & 57.5 & 1,300 & 16.1 & 6.77 & 42.4 & 0 & 21.1 & 0 & 13.8 & 0 & 1,740 & 0 & 1,740 \\
\hline Montana .......................... & 83.3 & 22.6 & 59.6 & 12.3 & 3.49 & 5.22 & 0 & 1.06 & 16.3 & 0.80 & 0 & 188 & 16.3 & 205 \\
\hline Nebraska ......................... & 218 & 19.0 & 5,420 & 89.7 & 10.5 & 42.0 & 0 & 0.06 & 6.41 & 16.8 & 0 & 5,810 & 6.41 & 5,820 \\
\hline Nevada ............................ & 142 & 35.8 & 972 & 4.94 & 11.6 & 1.02 & 0 & 187 & 11.3 & 7.04 & 70.9 & 1,360 & 82.2 & 1,440 \\
\hline New Hampshire ........... & 35.4 & 29.7 & 1.09 & 0.63 & 6.46 & 4.21 & 0 & 1.76 & 0 & 1.10 & 0 & 80.4 & 0 & 80.4 \\
\hline New Jersey ..................... & 379 & 90.7 & 55.1 & 0.88 & 9.78 & 30.0 & 0 & 1.48 & 0 & 2.08 & 0 & 569 & 0 & 569 \\
\hline New Mexico.................. & 184 & 24.6 & 1,050 & 29.8 & 18.7 & 3.40 & 0 & 39.4 & 89.4 & 6.13 & 0 & 1,350 & 89.4 & 1,440 \\
\hline New York ......................... & 614 & 187 & 17.8 & 16.8 & 11.0 & 29.4 & 0 & 5.64 & 0.95 & 7.30 & 0 & 890 & 0.95 & 890 \\
\hline North Carolina .............. & 158 & 169 & 83.8 & 51.9 & 13.3 & 15.0 & 0 & 30.0 & 0 & 0.18 & 0 & 520 & 0 & 520 \\
\hline North Dakota................. & 35.4 & 3.69 & 102 & 12.5 & 0 & 6.18 & 0 & 26.2 & 15.1 & 0.42 & 0 & 187 & 15.1 & 202 \\
\hline 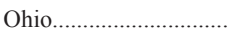 & 450 & 137 & 17.9 & 7.83 & 13.3 & 138 & 0 & 91.3 & 0 & 11.0 & 0 & 866 & 0 & 866 \\
\hline Oklahoma......................... & 102 & 30.3 & 787 & 27.4 & 0.06 & 7.27 & 0 & 4.22 & 155 & 1.59 & 0 & 960 & 155 & 1,110 \\
\hline 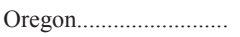 & 147 & 66.4 & 1,220 & 3.02 & 32.8 & 3.45 & 0 & 8.45 & 0 & 1.57 & 0 & 1,480 & 0 & 1,480 \\
\hline Pennsylvania ................. & 227 & 208 & 17.5 & 36.3 & 48.8 & 45.9 & 0 & 32.9 & 5.60 & 5.38 & 0 & 622 & 5.60 & 628 \\
\hline Rhode Island ..................... & 13.8 & 6.57 & 3.78 & 0.11 & 6.72 & 0.54 & 0 & 1.18 & 0 & 0 & 0 & 32.7 & 0 & 32.7 \\
\hline South Carolina ............. & 115 & 118 & 87.9 & 4.36 & 0.61 & 26.3 & 0 & 8.28 & 0 & 4.52 & 0 & 365 & 0 & 365 \\
\hline South Dakota.................. & 48.0 & 5.56 & 139 & 19.3 & 3.57 & 18.4 & 0 & 3.59 & 0 & 0 & 0 & 238 & 0 & 238 \\
\hline Tennessee ....................... & 256 & 42.8 & 36.4 & 12.0 & 11.7 & 51.6 & 0 & 17.1 & 0 & 2.18 & 0 & 430 & 0 & 430 \\
\hline 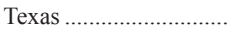 & 1,170 & 137 & 4,480 & 138 & 11.6 & 99.8 & 3.28 & 116 & 1,000 & 37.7 & 0 & 6,170 & 1,030 & 7,200 \\
\hline 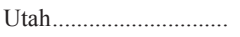 & 359 & 10.4 & 537 & 7.46 & 83.1 & 37.4 & 36.6 & 1.67 & 43.7 & 22.7 & 8.46 & 1,050 & 93.2 & 1,150 \\
\hline 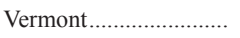 & 13.9 & 11.0 & 1.02 & 4.41 & 4.17 & 1.51 & 0 & 0.23 & 0 & 0.54 & 0 & 36.7 & 0 & 36.7 \\
\hline 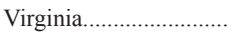 & 82.8 & 125 & 7.93 & 6.52 & 0.15 & 66.0 & 0 & 6.20 & 0 & 1.08 & 2.72 & 284 & 14.3 & 298 \\
\hline Washington........................ & 521 & 110 & 720 & 20.8 & 50.6 & 83.5 & 0 & 13.4 & 0 & 7.77 & 0 & 1,530 & 0 & 1,530 \\
\hline West Virginia.................... & 37.7 & 31.3 & 0.40 & 1.70 & 8.10 & 25.7 & 3.05 & 23.8 & 1.52 & 1.19 & 0 & 130 & 4.57 & 134 \\
\hline Wisconsin ........................ & 265 & 76.4 & 287 & 67.0 & 25.5 & 46.8 & 0 & 0.42 & 0 & 3.21 & 0 & 772 & 0 & 772 \\
\hline Wyoming ........................ & 54.5 & 8.93 & 537 & 6.17 & 4.92 & 5.83 & 0 & 33.1 & 96.8 & 1.31 & 0 & 652 & 96.8 & 748 \\
\hline Puerto Rico.................... & 66.6 & 0.52 & 41.6 & 4.23 & 0 & 3.67 & 0 & 1.84 & 0 & 1.82 & 0 & 120 & 0 & 120 \\
\hline U.S. Virgin Islands ...... & 0.91 & 1.23 & 0 & 0.01 & 0 & 0.52 & 0 & 0 & 0 & 0 & 0 & 2.67 & 0 & 2.67 \\
\hline TOTAL & 15,200 & 3,210 & 57,200 & 1,240 & 1,600 & 2,670 & 42.9 & 1,010 & 1,860 & 425 & 172 & 82,300 & 2,340 & 84,700 \\
\hline
\end{tabular}


Table 4B. Groundwater withdrawals by water-use category, 2015, in thousand acre-feet per year.

[Values may not sum to totals because of independent rounding. Values for public supply and aquaculture include fresh and saline-water withdrawals]

\begin{tabular}{|c|c|c|c|c|c|c|c|c|c|c|c|c|c|c|}
\hline \multirow[t]{2}{*}{ State } & \multirow{2}{*}{$\begin{array}{c}\begin{array}{c}\text { Public } \\
\text { supply }\end{array} \\
\text { Total } \\
\end{array}$} & \multirow{2}{*}{$\begin{array}{c}\text { Domes- } \\
\text { tic }\end{array}$} & \multirow{2}{*}{$\begin{array}{c}\begin{array}{c}\text { Irriga- } \\
\text { tion }\end{array} \\
\text { Fresh } \\
\end{array}$} & \multirow{2}{*}{$\begin{array}{l}\text { Live- } \\
\text { stock }\end{array}$} & \multirow{2}{*}{$\begin{array}{c}\begin{array}{c}\text { Aqua- } \\
\text { culture }\end{array} \\
\text { Total } \\
\end{array}$} & \multicolumn{2}{|c|}{ Industrial } & \multicolumn{2}{|c|}{ Mining } & \multicolumn{2}{|c|}{$\begin{array}{c}\text { Thermoelectric } \\
\text { power }\end{array}$} & \multicolumn{3}{|c|}{ Total } \\
\hline & & & & & & Fresh & Saline & Fresh & Saline & Fresh & Saline & Fresh & Saline & Total \\
\hline Alabama ........................... & 305 & 41.1 & 111 & 12.9 & 30.6 & 36.6 & 0 & 24.4 & 0 & 0 & 0 & 561 & 0 & 561 \\
\hline Alaska ................................ & 42.4 & 11.0 & 1.68 & 0.04 & 190 & 7.51 & 0 & 0 & 100 & 0.71 & 0 & 253 & 100 & 353 \\
\hline Arizona................................ & 638 & 26.9 & 2,210 & 43.6 & 26.7 & 6.86 & 0 & 76.6 & 0 & 64.7 & 0 & 3,090 & 0 & 3,090 \\
\hline Arkansas.............................. & 106 & 14.4 & 10,400 & 15.2 & 171 & 33.1 & 0 & 0.16 & 0 & 3.51 & 0 & 10,700 & 0 & 10,700 \\
\hline California ......................... & 2,650 & 121 & 15,500 & 92.0 & 276 & 446 & 0 & 33.9 & 305 & 22.0 & 39.4 & 19,100 & 403 & 19,500 \\
\hline Colorado.......................... & 122 & 39.6 & 1,460 & 25.4 & 17.9 & 4.73 & 0 & 5.87 & 27.1 & 3.40 & 0 & 1,680 & 27.1 & 1,710 \\
\hline Connecticut ....................... & 48.2 & 34.6 & 3.39 & 1.29 & 10.3 & 44.9 & 0 & 0.92 & 0 & 0 & 0 & 144 & 0 & 144 \\
\hline Delaware ......................... & 51.3 & 16.3 & 107 & 1.50 & 2.22 & 12.0 & 0 & 0.38 & 0 & 0.15 & 0 & 191 & 0 & 191 \\
\hline District of Columbia ... & 0 & 0 & 0 & 0 & 0 & 0 & 0 & 0 & 0 & 0 & 0 & 0 & 0 & 0 \\
\hline 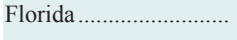 & 2,330 & 198 & 1,290 & 28.8 & 14.4 & 203 & 0 & 99.9 & 0 & 31.2 & 32.0 & 4,010 & 222 & 4,230 \\
\hline Georgia.......................... & 259 & 117 & 632 & 30.9 & 16.3 & 216 & 0 & 17.9 & 0 & 3.85 & 0 & 1,290 & 0 & 1,290 \\
\hline Hawaii ............................. & 283 & 0.74 & 82.7 & 0.57 & 9.62 & 0.27 & 0 & 1.00 & 0 & 1.66 & 22.7 & 379 & 22.7 & 402 \\
\hline Idaho ................................. & 276 & 78.6 & 5,490 & 46.2 & 51.8 & 50.4 & 0 & 1.51 & 0 & 2.01 & 0 & 6,000 & 0 & 6,000 \\
\hline Illinois & 411 & 103 & 245 & 40.6 & 5.86 & 148 & 0 & 16.4 & 23.5 & 4.72 & 0 & 976 & 23.5 & 999 \\
\hline Indiana................................. & 380 & 143 & 104 & 29.4 & 7.24 & 89.6 & 0 & 6.19 & 0 & 23.3 & 0 & 783 & 0 & 783 \\
\hline 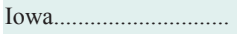 & 352 & 35.8 & 36.1 & 139 & 8.36 & 104 & 0 & 1.21 & 0 & 30.2 & 0 & 706 & 0 & 706 \\
\hline Kansas ............................... & 153 & 19.8 & 2,870 & 93.6 & 3.54 & 34.2 & 0 & 6.10 & 0 & 8.76 & 0 & 3,180 & 6.93 & 3,190 \\
\hline Kentucky ........................... & 98.0 & 16.2 & 3.18 & 2.32 & 0.65 & 86.6 & 0 & 19.8 & 0 & 5.56 & 0 & 232 & 0 & 232 \\
\hline Louisiana .............................. & 397 & 44.1 & 807 & 3.54 & 378 & 280 & 0 & 1.85 & 0 & 41.4 & 0 & 1,950 & 0 & 1,950 \\
\hline Maine ………………......... & 30.8 & 35.5 & 5.67 & 1.73 & 12.2 & 6.54 & 0 & 1.63 & 0 & 1.03 & 0 & 95.0 & 0 & 95.0 \\
\hline Maryland .......................... & 105 & 128 & 54.1 & 6.56 & 6.63 & 12.2 & 0 & 15.3 & 0 & 2.58 & 0 & 330 & 0 & 330 \\
\hline Massachusetts ................ & 224 & 39.4 & 128 & 0.72 & 9.11 & 17.0 & 0 & 6.18 & 0 & 1.23 & 0 & 426 & 0 & 426 \\
\hline Michigan ……..................... & 235 & 210 & 293 & 24.0 & 1.19 & 72.4 & 0 & 18.0 & 0.65 & 6.29 & 0 & 859 & 0.65 & 860 \\
\hline Minnesota......................... & 377 & 92.2 & 272 & 66.0 & 1.93 & 54.9 & 0 & 4.07 & 0 & 1.98 & 0 & 870 & 0 & 870 \\
\hline Mississippi ....................... & 389 & 54.0 & 1,840 & 7.68 & 98.1 & 81.6 & 0 & 9.20 & 7.38 & 37.8 & 7.12 & 2,520 & 14.5 & 2,530 \\
\hline Missouri ........................... & 316 & 64.4 & 1,460 & 18.1 & 7.59 & 47.5 & 0 & 23.6 & 0 & 15.4 & 0 & 1,950 & 0 & 1,950 \\
\hline Montana .......................... & 93.4 & 25.3 & 66.8 & 13.8 & 3.91 & 5.85 & 0 & 1.19 & 18.3 & 0.90 & 0 & 211 & 18.3 & 229 \\
\hline Nebraska ........................... & 244 & 21.3 & 6,070 & 101 & 11.8 & 47.0 & 0 & 0.07 & 7.19 & 18.9 & 0 & 6,520 & 7.19 & 6,520 \\
\hline Nevada ................................. & 159 & 40.1 & 1,090 & 5.54 & 13.0 & 1.14 & 0 & 210 & 12.6 & 7.89 & 79.5 & 1,530 & 92.2 & 1,620 \\
\hline New Hampshire ............ & 39.7 & 33.3 & 1.22 & 0.71 & 7.24 & 4.72 & 0 & 1.97 & 0 & 1.23 & 0 & 90.1 & 0 & 90.1 \\
\hline New Jersey ........................ & 424 & 102 & 61.8 & 0.99 & 11.0 & 33.7 & 0 & 1.66 & 0 & 2.33 & 0 & 637 & 0 & 637 \\
\hline New Mexico......................... & 206 & 27.6 & 1,180 & 33.4 & 20.9 & 3.81 & 0 & 44.1 & 100 & 6.87 & 0 & 1,520 & 100 & 1,620 \\
\hline New York ............................... & 688 & 210 & 20.0 & 18.8 & 12.3 & 32.9 & 0 & 6.32 & 1.06 & 8.18 & 0 & 997 & 1.06 & 998 \\
\hline North Carolina .............. & 177 & 189 & 93.9 & 58.2 & 14.9 & 16.8 & 0 & 33.7 & 0 & 0.20 & 0 & 583 & 0 & 583 \\
\hline North Dakota...................... & 39.6 & 4.14 & 115 & 14.0 & 0 & 6.93 & 0 & 29.3 & 16.9 & 0.47 & 0 & 209 & 16.9 & 226 \\
\hline Ohio................................... & 504 & 153 & 20.0 & 8.78 & 14.9 & 155 & 0 & 102 & 0 & 12.3 & 0 & 970 & 0 & 970 \\
\hline Oklahoma ......................... & 114 & 34.0 & 882 & 30.7 & 0.07 & 8.15 & 0 & 4.73 & 173 & 1.78 & 0 & 1,080 & 173 & 1,250 \\
\hline 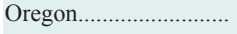 & 165 & 74.4 & 1,360 & 3.39 & 36.8 & 3.87 & 0 & 9.47 & 0 & 1.76 & 0 & 1,660 & 0 & 1,660 \\
\hline Pennsylvania ..................... & 255 & 233 & 19.6 & 40.7 & 54.7 & 51.5 & 0 & 36.9 & 6.28 & 6.03 & 0 & 698 & 6.28 & 704 \\
\hline 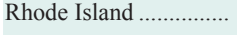 & 15.4 & 7.36 & 4.24 & 0.12 & 7.53 & 0.61 & 0 & 1.32 & 0 & 0 & 0 & 36.6 & 0 & 36.6 \\
\hline 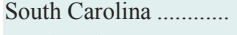 & 129 & 133 & 98.5 & 4.89 & 0.68 & 29.5 & 0 & 9.28 & 0 & 5.07 & 0 & 410 & 0 & 410 \\
\hline South Dakota..................... & 53.8 & 6.23 & 156 & 21.6 & 4.00 & 20.6 & 0 & 4.02 & 0 & 0 & 0 & 267 & 0 & 267 \\
\hline Tennessee ......................... & 287 & 48.0 & 40.8 & 13.4 & 13.1 & 57.8 & 0 & 19.2 & 0 & 2.44 & 0 & 482 & 0 & 482 \\
\hline Texas ………………............ & 1,320 & 153 & 5,020 & 155 & 13.0 & 112 & 3.68 & 130 & 1,130 & 42.2 & 0 & 6,920 & 1,150 & 8,070 \\
\hline Utah ... - & 403 & 11.6 & 602 & 8.36 & 93.1 & 41.9 & 41.0 & 1.87 & 49.0 & 25.5 & 9.48 & 1,180 & 105 & 1,290 \\
\hline Vermont.......................... & 15.6 & 12.3 & 1.14 & 4.94 & 4.67 & 1.69 & 0 & 0.26 & 0 & 0.61 & 0 & 41.2 & 0 & 41.2 \\
\hline Virginia................................. & 92.9 & 140 & 8.89 & 7.31 & 0.17 & 74.0 & 0 & 6.95 & 0 & 1.21 & 3.05 & 318 & 16.0 & 334 \\
\hline Washington.......................... & 584 & 123 & 807 & 23.4 & 56.8 & 93.6 & 0 & 15.1 & 0 & 8.71 & 0 & 1,710 & 0 & 1,710 \\
\hline West Virginia..................... & 42.2 & 35.1 & 0.45 & 1.91 & 9.08 & 28.8 & 3.42 & 26.7 & 1.70 & 1.33 & 0 & 146 & 5.12 & 151 \\
\hline 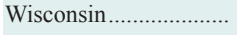 & 297 & 85.7 & 322 & 75.2 & 28.6 & 52.4 & 0 & 0.47 & 0 & 3.60 & 0 & 865 & 0 & 865 \\
\hline Wyoming ............................. & 61.1 & 10.0 & 602 & 6.92 & 5.52 & 6.54 & 0 & 37.1 & 108 & 1.47 & 0 & 731 & 108 & 839 \\
\hline Puerto Rico......................... & 74.7 & 0.58 & 46.6 & 4.74 & 0 & 4.11 & 0 & 2.06 & 0 & 2.04 & 0 & 135 & 0 & 135 \\
\hline U.S. Virgin Islands ....... & 1.02 & 1.38 & 0 & 0.01 & 0 & 0.58 & 0 & 0 & 0 & 0 & 0 & 2.99 & 0 & 2.99 \\
\hline TOTAL & 7,100 & 3,600 & 64,100 & 1,390 & 800 & ,990 & 48.1 & 1,130 & 2,090 & 477 & 193 & 92,300 & 2,620 & 94,900 \\
\hline
\end{tabular}


Public supply refers to water withdrawn by public and private water suppliers that provide water to at least 25 people or have a minimum of 15 connections. Public-supply water is delivered to users for domestic, commercial, thermoelectric, irrigation, and industrial purposes; it also is used for public services and system losses.

Data on population, public supply and self-served domestic populations, public-supply withdrawals with deliveries to domestic users, and self-supplied domestic withdrawals for 2015 were published by the USGS in Dieter and Maupin (2017) and Dieter and others (2017). Data and interpretations of the data presented in Dieter and Maupin (2017) and Dieter and others (2017) are superseded by this report and the concurrent data release (Dieter and others, 2018). Specifically, some county-level data have been revised for public-supply and self-supplied domestic populations (Florida and Georgia), public-supply withdrawals (Georgia, Nevada, Tennessee, and Utah), and self-supplied domestic withdrawals (Utah and U.S. Virgin Islands). Changes to public-supply deliveries for domestic uses also were made (Utah). Changes to publicsupply population served, withdrawals (public supply and self-supplied domestic), and deliveries have caused changes to calculated values of county per capita rates (Florida, Utah, U.S. Virgin Islands).

In some States, publicsupply water sources include desalinated seawater or brackish groundwater that has been treated to reduce dissolved solids. A combined total of 7.21 Mgal/d saline surfacewater withdrawals for publicsupply use were reported for the U.S. Virgin Islands, Texas, Florida, and Massachusetts. A combined total of $263 \mathrm{Mgal} / \mathrm{d}$ saline groundwater withdrawals for public-supply use were identified for Florida, California, Texas, Virginia, Kansas, and Utah. Saline withdrawals for public supply were identified for only eight States and represent less than 1 percent of total public-supply withdrawals; therefore, they are not listed separately in table 5 but are included in the estimated withdrawals.

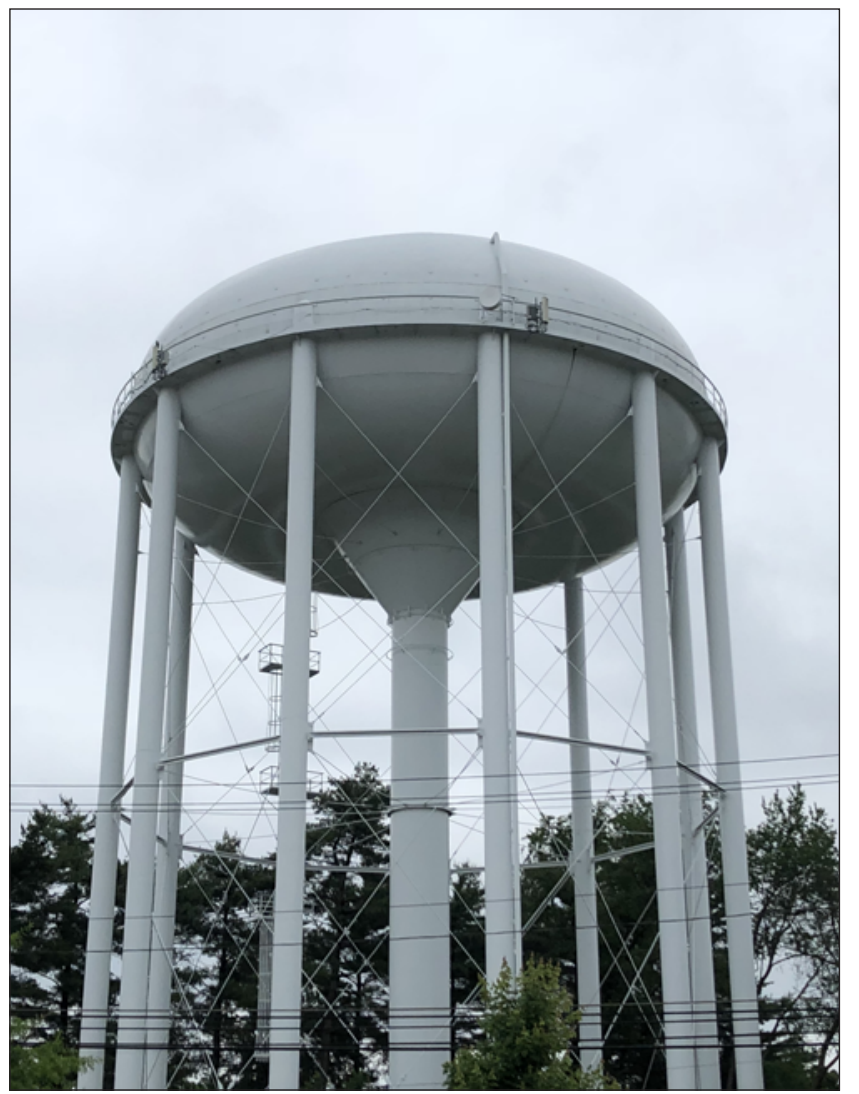

Public-supply water tower in Baltimore County, Maryland.
Discussions of public supply withdrawals below include these saline withdrawals.

A total of 39,000 Mgal/d (table 5), or 43,700 thousand acre-ft/yr (table $2 B$ ), of water were withdrawn for public supply in 2015. This amount is 7 percent less than the estimated amount of water withdrawn for public supply in 2010 , continuing the decline in public-supply withdrawals observed from 2005 to 2010 (Maupin and others, 2014). Total publicsupply withdrawals in 2015 were at the lowest levels since 1995 (approximately 40,200 Mgal/d) (Maupin and others, 2014). Public supply represents about 14 percent of total freshwater withdrawals and 21 percent of all withdrawals, excluding thermoelectric power.

An estimated 283 million people relied on public-supply water for household use in 2015. This number represents about 87 percent of the total United States population. About 33 percent of all public-supply withdrawals were made in the four States with the largest populations: California, Texas, New York, and Florida (fig. 4). Populations in the District of Columbia and Puerto Rico are supplied almost completely by public-supply systems, whereas only one-half of the populations in the U.S. Virgin Islands and Maine are supplied by public-supply systems. Sixty-one percent of water withdrawn for public supply in 2015 was from surface sources, such as lakes and streams; the other 39 percent was from groundwater.

Some States in the Nation, because of population, withdraw more than 1,000 Mgal/d of water for distribution or rely primarily on either surface water or groundwater as a source for public-supply potable water. Five StatesCalifornia, New York, Texas, Pennsylvania, and Illinoiseach withdrew more than $1,000 \mathrm{Mgal} / \mathrm{d}$ of surface water for public supply in 2015 and together accounted for 36 percent of the total surface-water withdrawals for public supply. In 36 States, including Puerto Rico and the U.S. Virgin Islands, surface-water sources provided more than one-half of 


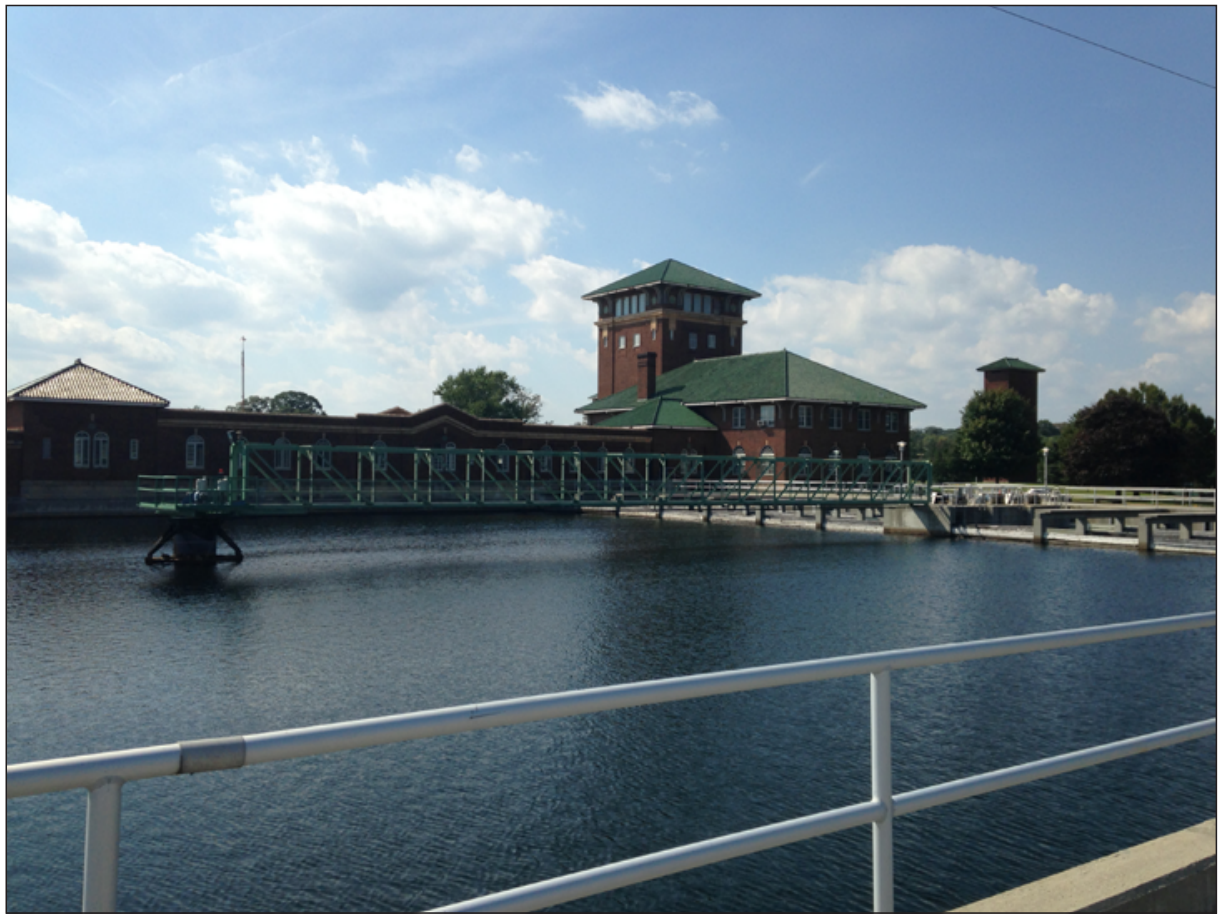

Water treatment plant, Baltimore, Maryland. Photograph by Cheryl A. Dieter, USGS.

the total public-supply withdrawals. Three States-California, Florida, and Texas - each withdrew more than 1,000 Mgal/d of groundwater for public supply in 2015 and accounted for 37 percent of total groundwater withdrawals for public supply (table 5; fig. 4). States that relied on groundwater for 75 percent or more of their public-supply withdrawals were Hawaii, Idaho, Florida, Mississippi, Iowa, and Nebraska.

Most of the public-supply withdrawals are delivered to customers for domestic, commercial, and industrial needs. Part of the total is used for public services, such as public pools, parks, firefighting, water and wastewater treatment, and municipal buildings, and some is unaccounted for because of leaks, flushing, tower maintenance, and other system losses. Public-supply deliveries to commercial, industrial, and thermoelectric-power users have not been reported by the USGS NWUSP since 1995. However, public-supply deliveries to domestic users are included in these data, which when combined with the self-supplied domestic withdrawals, completes the amount of water withdrawn directly for human consumption. Domestic deliveries represent the largest single component of public-supply withdrawals, averaging 60 percent of the total nationally. Estimates of public-supply deliveries for domestic use, by State, representing indoor and outdoor water uses at occupied residences, are identified in table 5. Estimates for commercial and industrial deliveries, public use, and system losses were not available for all States and, therefore, are included in table 5 as an aggregate number ("All other uses and system losses"). Some States reported public-supply deliveries to thermoelectric powerplants for 2015, and these data are presented in the "Thermoelectric Power" section of this report and are included in table 5.

Methods for estimating public-supply withdrawals, source of water, population served, and domestic deliveries varied by State. Common sources of information about withdrawals by source included data collected from water suppliers by State water regulatory agencies or through surveys. Estimates of the population served by public supply were derived using various sources, including reports from State agencies, the U.S. Environmental Protection Agency (EPA) Safe Drinking Water Information System (SDWIS) database, U.S. Census data, and information on service connections from public suppliers. Methods for estimating domestic deliveries included surveys of public-supply sales information, calculations using coefficients for per capita use, and development of average percentages of deliveries to various customer categories (Bradley, 2017). 
Table 5. Public-supply water withdrawals, 2015.

[Values may not sum to totals because of independent rounding. Values for public supply include fresh and saline-water withdrawals. Abbreviations: Mgal/d, million gallons per day; _, not estimated; n/a, not applicable]

\begin{tabular}{|c|c|c|c|c|c|c|c|c|c|}
\hline \multirow{3}{*}{ State } & \multicolumn{3}{|c|}{ Population (thousands) } & \multicolumn{3}{|c|}{ Withdrawals (Mgal/d) } & \multicolumn{3}{|c|}{ Public-supply deliveries } \\
\hline & \multirow[b]{2}{*}{ Total } & \multicolumn{2}{|c|}{ Served by public supply } & \multicolumn{2}{|c|}{ By source } & \multirow[b]{2}{*}{ Total } & \multirow[b]{2}{*}{$\begin{array}{c}\text { Domestic use } \\
(\text { Mgal/d) }\end{array}$} & \multirow{2}{*}{$\begin{array}{l}\text { Thermoelectric- } \\
\text { power generation } \\
\text { use (Mgal/d) }\end{array}$} & \multirow{2}{*}{$\begin{array}{l}\text { All other uses and } \\
\text { system losses } \\
\text { (Mgal/d) }\end{array}$} \\
\hline & & Population & $\begin{array}{c}\text { Population } \\
\text { (percent) }\end{array}$ & $\begin{array}{l}\text { Ground- } \\
\text { water }\end{array}$ & $\begin{array}{c}\text { Surface } \\
\text { water }\end{array}$ & & & & \\
\hline 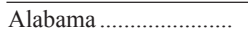 & 4,860 & 4,320 & 89 & 272 & 490 & 762 & 320 & - & 441 \\
\hline Alaska ................................... & 738 & 550 & 74 & 37.8 & 61.4 & 99.2 & 61.6 & 0.60 & 37.0 \\
\hline Arizona.................................. & 6,830 & 6,610 & 97 & 569 & 626 & 1,200 & 963 & 0.50 & 231 \\
\hline Arkansas.............................. & 2,980 & 2,830 & 95 & 94.2 & 269 & 363 & 252 & - & 111 \\
\hline California & 39,100 & 37,700 & 96 & 2,370 & 2,780 & 5,150 & 3,230 & 38.9 & 1,880 \\
\hline Colorado............................ & 5,460 & 5,170 & 95 & 109 & 735 & 844 & 637 & 13.7 & 194 \\
\hline Connecticut .................... & 3,590 & 2,730 & 76 & 43.0 & 197 & 240 & 96.0 & 4.65 & 139 \\
\hline Delaware ……....................... & 946 & 764 & 81 & 45.8 & 40.6 & 86.4 & 61.1 & - & 25.2 \\
\hline District of Columbia ... & 672 & 672 & 100 & 0 & 0 & 0 & 44.8 & 0 & $\mathrm{n} / \mathrm{a}$ \\
\hline Florida ................................ & 20,300 & 17,800 & 88 & 2,080 & 307 & 2,380 & 1,500 & 8.88 & 874 \\
\hline 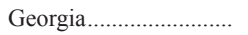 & 10,200 & 8,700 & 85 & 231 & 839 & 1,070 & 661 & - & 409 \\
\hline Hawaii ................................ & 1,430 & 1,380 & 96 & 252 & 14.6 & 267 & 198 & - & 68.9 \\
\hline Idaho .................................. & 1,650 & 1,260 & 76 & 247 & 29.2 & 276 & 234 & - & 41.7 \\
\hline Illinois ............................... & 12,900 & 11,700 & 91 & 367 & 1,110 & 1,480 & 937 & - & 539 \\
\hline Indiana............................... & 6,620 & 4,940 & 75 & 339 & 288 & 628 & 376 & - & 252 \\
\hline Iowa.................................... & 3,120 & 2,630 & 84 & 314 & 76.8 & 390 & 171 & 0.16 & 219 \\
\hline Kansas ................................. & 2,910 & 2,760 & 95 & 137 & 214 & 351 & 175 & 0.25 & 176 \\
\hline Kentucky .......................... & 4,430 & 3,980 & 90 & 87.4 & 465 & 553 & 279 & - & 274 \\
\hline Louisiana ............................ & 4,670 & 4,180 & 89 & 354 & 355 & 709 & 497 & - & 212 \\
\hline Maine ….............................. & 1,330 & 669 & 50 & 27.5 & 57.5 & 85.0 & 35.5 & 2.05 & 47.4 \\
\hline Maryland ............................ & 6,010 & 4,580 & 76 & 93.9 & 656 & 750 & 320 & - & 430 \\
\hline Massachusetts ............... & 6,790 & 6,180 & 91 & 199 & 449 & 648 & 347 & 47.0 & 254 \\
\hline Michigan ............................. & 9,920 & 7,330 & 74 & 209 & 821 & 1,030 & 481 & - & 550 \\
\hline Minnesota........................... & 5,490 & 4,310 & 79 & 336 & 179 & 515 & 236 & - & 279 \\
\hline 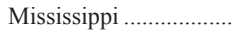 & 2,990 & 2,560 & 85 & 347 & 53.4 & 400 & 251 & 0.01 & 149 \\
\hline Missouri ............................ & 6,080 & 5,260 & 86 & 282 & 516 & 797 & 482 & 1.64 & 313 \\
\hline Montana ……....................... & 1,030 & 728 & 71 & 83.3 & 69.9 & 153 & 86.2 & - & 67.0 \\
\hline Nebraska ............................ & 1,900 & 1,720 & 91 & 218 & 57.2 & 275 & 129 & - & 146 \\
\hline Nevada ............................... & 2,890 & 2,700 & 93 & 142 & 390 & 531 & 330 & 0.49 & 201 \\
\hline New Hampshire ............ & 1,330 & 833 & 63 & 35.4 & 60.1 & 95.5 & 50.0 & 0.53 & 45.0 \\
\hline New Jersey ....................... & 8,960 & 7,990 & 89 & 379 & 797 & 1,180 & 626 & 0.71 & 549 \\
\hline New Mexico................... & 2,090 & 1,790 & 86 & 184 & 78.3 & 262 & 145 & 0.26 & 116 \\
\hline New York .......................... & 19,800 & 17,300 & 87 & 614 & 1,810 & 2,420 & 1,230 & 17.1 & 1,180 \\
\hline North Carolina .............. & 10,000 & 7,640 & 76 & 158 & 780 & 938 & 534 & 8.33 & 395 \\
\hline North Dakota......................... & 757 & 711 & 94 & 35.4 & 48.8 & 84.2 & 56.8 & 0.05 & 27.3 \\
\hline 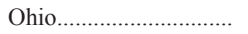 & 11,600 & 9,750 & 84 & 450 & 857 & 1,310 & 589 & 4.19 & 713 \\
\hline Oklahoma ........................... & 3,910 & 3,550 & 91 & 102 & 509 & 611 & 247 & 6.96 & 357 \\
\hline Oregon.............................. & 4,030 & 3,400 & 84 & 147 & 420 & 567 & 355 & 4.53 & 207 \\
\hline Pennsylvania .................. & 12,800 & 9,330 & 73 & 227 & 1,160 & 1,390 & 522 & - & 869 \\
\hline Rhode Island .................... & 1,060 & 943 & 89 & 13.8 & 83.7 & 97.5 & 56.0 & 1.44 & 40.0 \\
\hline South Carolina ................... & 4,900 & 3,710 & 76 & 115 & 518 & 633 & 371 & 3.93 & 258 \\
\hline South Dakota................... & 881 & 752 & 85 & 48.0 & 24.0 & 72.0 & 43.7 & - & 28.2 \\
\hline Tennessee .......................... & 6,600 & 6,010 & 91 & 256 & 594 & 850 & 486 & - & 363 \\
\hline Texas ................................. & 27,500 & 26,200 & 95 & 1,170 & 1,710 & 2,890 & 2,120 & 48.3 & 712 \\
\hline Utah.................................. & 3,000 & 2,930 & 98 & 359 & 267 & 627 & 496 & 1.33 & 129 \\
\hline Vermont.............................. & 626 & 382 & 61 & 13.9 & 28.8 & 42.7 & 16.6 & - & 26.1 \\
\hline 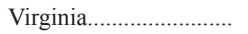 & 8,380 & 6,820 & 81 & 82.8 & 614 & 697 & 546 & 0.29 & 151 \\
\hline Washington......................... & 7,170 & 6,150 & 86 & 521 & 345 & 867 & 631 & 1.63 & 234 \\
\hline West Virginia.................. & 1,840 & 1,450 & 79 & 37.7 & 147 & 185 & 116 & 0.62 & 68.7 \\
\hline Wisconsin........................... & 5,770 & 4,170 & 72 & 265 & 214 & 479 & 240 & 0.23 & 239 \\
\hline Wyoming.............................. & 586 & 467 & 80 & 54.5 & 46.8 & 101 & 82.3 & - & 19.0 \\
\hline Puerto Rico........................ & 3,470 & 3,470 & 100 & 66.6 & 510 & 576 & 338 & 0.97 & 237 \\
\hline U.S. Virgin Islands ...... & 104 & 51.9 & 50 & 0.91 & 3.36 & 4.27 & 2.74 & - & 1.53 \\
\hline TOTAL & 325,000 & 283,000 & 87 & 15,200 & 23,800 & 39,000 & 23,300 & 220 & 15,500 \\
\hline
\end{tabular}



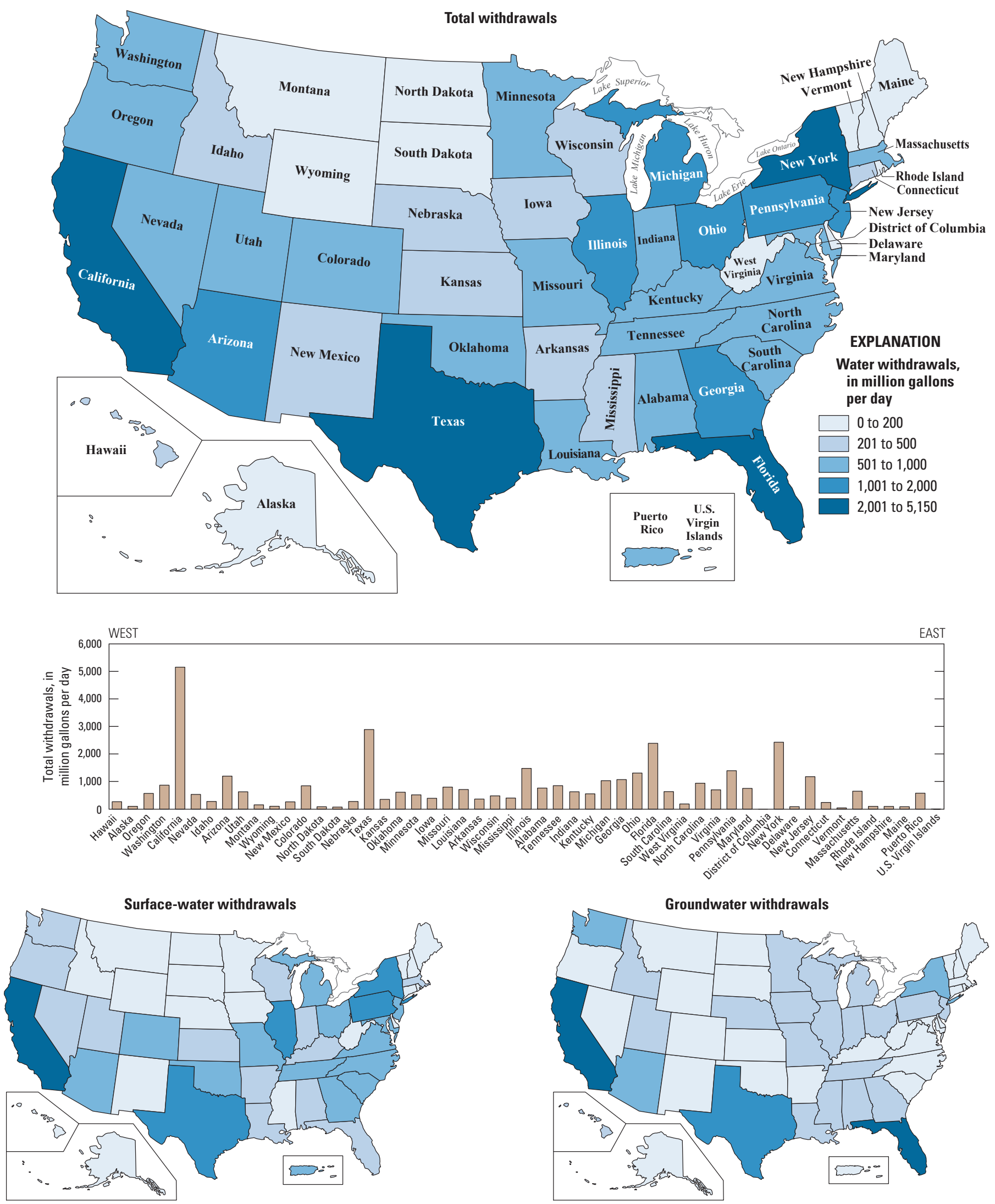

Figure 4. Public-supply withdrawals by source and State, 2015. 


\section{Domestic}

\section{3,260 million gallons per day (self-supplied)} 23,300 million gallons per day (public-supply deliveries)

Domestic water use includes indoor and outdoor uses at residences. Common indoor water uses are drinking, food preparation, washing clothes and dishes, bathing, and flushing toilets. Common outdoor uses are watering lawns and gardens or maintaining pools, ponds, or other landscape features in a domestic environment. Domestic water is either self-supplied or provided by public suppliers. Water for self-supplied domestic use is typically withdrawn from a private source, such as a well, or captured as rainwater in a cistern. Domestic deliveries are provided to homes by public suppliers. The proportion of total domestic water use from public-supply deliveries ( 88 percent) and self-supplied domestic withdrawals (12 percent) in the United States is shown in figure 5.

The estimated self-supplied and public-supplied populations in each State are listed in table 6, as well as the amounts used by each segment of the population for domestic needs and the respective per capita use in gallons per day (gallons per capita per day, GPCD). Domestic self-supplied withdrawals and public-supplied deliveries are combined in table 6 to show the total estimated domestic use in 2015 and the total per capita use in gallons per day calculated for all domestic use.

An estimated 42.5 million people in the United States, or 13 percent of the population, provided their own water for domestic use in 2015. These selfsupplied withdrawals were estimated to be 3,260 Mgal/d (3,650 thousand acre-ft/yr), or about 1 percent of total withdrawals for all uses in 2015.

Nearly all (98 percent) of these selfsupplied withdrawals were from fresh groundwater sources. Self-supplied domestic withdrawals are rarely metered or reported; typically, this usage is calculated by multiplying an estimate of the population not served by public supply by a coefficient for daily per capita use. For some States, these coefficients were county-specific averages derived from observed residential water use and population estimates in nearby areas served by public suppliers. Other States used the same coefficient for all counties, commonly one used by State regulatory or planning agencies.
Self-supplied domestic per capita use ranged from 36 GPCD in Connecticut to 186 GPCD in Nevada. Generally, per capita use is least in the Northern and Eastern States and greatest in the Mountain and Western States where outdoor watering is more common. The national average self-supplied domestic per capita use in 2015 was 77 GPCD (table 6).

Most people in the United States used water provided by public suppliers. Domestic deliveries by public water suppliers totaled 23,300 Mgal/d in 2015 and represented water provided to 283 million people at single-family and multifamily dwellings. The District of Columbia and Puerto Rico have populations that are almost entirely supplied by publicsupply systems. The District of Columbia had zero self-supplied domestic withdrawals estimated in 2015, and less than $1 \mathrm{Mgal} / \mathrm{d}$ was estimated for Puerto Rico in 2015. Per capita water use for domestic deliveries ranged from 35 GPCD in Connecticut to 186 GPCD in Idaho. The national average was 82 GPCD for public-supplied domestic water use in 2015. This per capita usage is less than the rate of 101 GPCD observed in 1995, 100 GPCD in 2005, and 88 GPCD in 2010. Domestic deliveries from public supply were not compiled nationally in 2000 .

Combined self-supplied domestic withdrawals and public-supply deliveries totaled 26,600 Mgal/d in 2015, with 23,300 Mgal/d from public-supply deliveries (88 percent) and 3,260 Mgal/d from self-supplied withdrawals (12 percent), and the national average per capita use was 82 GPCD. The corresponding average per capita use for total domestic use in 2005 was 98 GPCD, and in 2010, it was 87 GPCD. The geographic distribution of total domestic water use by State is shown in figure $6 \mathrm{~A}$. The self-supplied domestic population in each State, in thousands of people and as a percentage of total State population, is shown in figure $6 B$. Self-supplied domestic populations were largest in Pennsylvania, Michigan, and New York. States where nearly one-half the population has a self-supplied water source were U.S. Virgin Islands and Maine. 
Table 6. Domestic water withdrawals and deliveries, 2015.

[Values may not sum to totals because of independent rounding. Abbreviations: gal/d, gallons per day; Mgal/d, million gallons per day]

\begin{tabular}{|c|c|c|c|c|c|c|c|c|c|c|c|c|}
\hline \multirow{3}{*}{ State } & \multicolumn{6}{|c|}{ Self-supplied domestic } & \multicolumn{3}{|c|}{ Public supply } & \multicolumn{3}{|c|}{ Total domestic use } \\
\hline & \multirow{2}{*}{$\begin{array}{c}\text { Self- } \\
\text { supplied } \\
\text { population } \\
\text { (thousands) }\end{array}$} & \multirow{2}{*}{$\begin{array}{c}\text { Percent } \\
\text { of total } \\
\text { popula- } \\
\text { tion }\end{array}$} & \multicolumn{3}{|c|}{$\begin{array}{l}\text { Withdrawals (Mgal/d) } \\
\text { By source }\end{array}$} & \multirow{2}{*}{$\begin{array}{c}\text { Self- } \\
\text { supplied per } \\
\text { capita use } \\
\text { (gal/d) }\end{array}$} & \multirow{2}{*}{$\begin{array}{c}\text { Population } \\
\text { served } \\
\text { (thousands) }\end{array}$} & \multirow{2}{*}{$\begin{array}{c}\text { Water } \\
\text { deliveries } \\
\text { (Mgal/d) }\end{array}$} & \multirow{2}{*}{$\begin{array}{c}\text { Public- } \\
\text { supply per } \\
\text { capita use } \\
\text { (gal/d) }\end{array}$} & \multirow{2}{*}{$\begin{array}{c}\text { Total } \\
\text { population } \\
\text { (thousands) }\end{array}$} & \multirow{2}{*}{$\begin{array}{c}\text { Water use } \\
\text { (withdrawals } \\
\text { and } \\
\text { deliveries) } \\
\text { (Mgal/d) }\end{array}$} & \multirow{2}{*}{$\begin{array}{c}\text { Total } \\
\text { domes- } \\
\text { tic per } \\
\text { capita use } \\
\text { (gal/d) }\end{array}$} \\
\hline & & & $\begin{array}{c}\text { Ground- } \\
\text { water }\end{array}$ & $\begin{array}{l}\text { Surface } \\
\text { water }\end{array}$ & Total & & & & & & & \\
\hline Alabama .............................. & 539 & 11 & 36.7 & 0 & 36.7 & 68 & 4,320 & 320 & 74 & 4,860 & 357 & 73 \\
\hline Alaska ................................. & 189 & 26 & 9.83 & 1.59 & 11.4 & 60 & 550 & 61.6 & 112 & 738 & 73.0 & 99 \\
\hline Arizona............................... & 218 & 3 & 24.0 & 0 & 24.0 & 110 & 6,610 & 963 & 146 & 6,830 & 987 & 145 \\
\hline Arkansas............................ & 144 & 5 & 12.8 & 0 & 12.8 & 89 & 2,830 & 252 & 89 & 2,980 & 265 & 89 \\
\hline California ....................... & 1,440 & 4 & 108 & 18.5 & 127 & 88 & 37,700 & 3,230 & 86 & 39,100 & 3,350 & 86 \\
\hline Colorado............................. & 286 & 5 & 35.4 & 0 & 35.4 & 123 & 5,170 & 637 & 123 & 5,460 & 672 & 123 \\
\hline Connecticut ....................... & 861 & 24 & 30.8 & 0 & 30.8 & 36 & 2,730 & 96.0 & 35 & 3,590 & 127 & 35 \\
\hline Delaware ............................ & 182 & 19 & 14.5 & 0 & 14.5 & 80 & 764 & 61.1 & 80 & 946 & 75.7 & 80 \\
\hline District of Columbia ... & 0 & 0 & 0 & 0 & 0 & 0 & 672 & 44.8 & 67 & 672 & 44.8 & 67 \\
\hline Florida ........................... & 2,460 & 12 & 177 & 0 & 177 & 72 & 17,800 & 1,500 & 84 & 20,300 & 1,680 & 83 \\
\hline 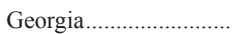 & 1,510 & 15 & 104 & 0 & 104 & 69 & 8,700 & 661 & 76 & 10,200 & 765 & 75 \\
\hline Hawaii ................................. & 56.3 & 4 & 0.66 & 7.44 & 8.10 & 144 & 1,380 & 198 & 144 & 1,430 & 206 & 144 \\
\hline Idaho ................................. & 396 & 24 & 70.2 & 0 & 70.2 & 177 & 1,260 & 234 & 186 & 1,650 & 304 & 184 \\
\hline 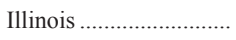 & 1,150 & 9 & 92.1 & 0 & 92.1 & 80 & 11,700 & 937 & 80 & 12,900 & 1,030 & 80 \\
\hline 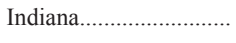 & 1,680 & 25 & 127 & 0 & 127 & 76 & 4,940 & 376 & 76 & 6,620 & 503 & 76 \\
\hline Iowa................................... & 494 & 16 & 32.0 & 0 & 32.0 & 65 & 2,630 & 171 & 65 & 3,120 & 203 & 65 \\
\hline Kansas .................................. & 149 & 5 & 17.7 & 0 & 17.7 & 118 & 2,760 & 175 & 63 & 2,910 & 192 & 66 \\
\hline 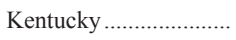 & 441 & 10 & 14.5 & 7.63 & 22.1 & 50 & 3,980 & 279 & 70 & 4,430 & 301 & 68 \\
\hline Louisiana............................. & 492 & 11 & 39.3 & 0 & 39.3 & 80 & 4,180 & 497 & 119 & 4,670 & 537 & 115 \\
\hline Maine & 660 & 50 & 31.6 & 0 & 31.6 & 48 & 669 & 35.5 & 53 & 1,330 & 67.1 & 51 \\
\hline Maryland ........................... & 1,420 & 24 & 114 & 0 & 114 & 80 & 4,580 & 320 & 70 & 6,010 & 433 & 72 \\
\hline Massachusetts ................ & 616 & 9 & 35.1 & 0 & 35.1 & 57 & 6,180 & 347 & 56 & 6,790 & 382 & 56 \\
\hline Michigan ........................... & 2,600 & 26 & 187 & 0 & 187 & 72 & 7,330 & 481 & 66 & 9,920 & 668 & 67 \\
\hline Minnesota.......................... & 1,180 & 21 & 82.2 & 0 & 82.2 & 70 & 4,310 & 236 & 55 & 5,490 & 318 & 58 \\
\hline Mississippi ....................... & 435 & 15 & 48.1 & 0 & 48.1 & 111 & 2,560 & 251 & 98 & 2,990 & 299 & 100 \\
\hline Missouri …….................... & 821 & 14 & 57.5 & 0 & 57.5 & 70 & 5,260 & 482 & 92 & 6,080 & 540 & 89 \\
\hline Montana ............................... & 305 & 29 & 22.6 & 1.12 & 23.7 & 78 & 728 & 86.2 & 118 & 1,030 & 110 & 106 \\
\hline Nebraska ........................... & 171 & 9 & 19.0 & 0 & 19.0 & 111 & 1,720 & 129 & 75 & 1,900 & 148 & 78 \\
\hline Nevada .............................. & 193 & 7 & 35.8 & 0 & 35.8 & 186 & 2,700 & 330 & 122 & 2,890 & 365 & 126 \\
\hline New Hampshire ........... & 497 & 37 & 29.7 & 0 & 29.7 & 60 & 833 & 50.0 & 60 & 1,330 & 79.7 & 60 \\
\hline New Jersey ........................ & 966 & 11 & 90.7 & 0 & 90.7 & 94 & 7,990 & 626 & 78 & 8,960 & 717 & 80 \\
\hline New Mexico....................... & 292 & 14 & 24.6 & 0 & 24.6 & 84 & 1,790 & 145 & 81 & 2,090 & 170 & 81 \\
\hline New York .......................... & 2,500 & 13 & 187 & 0 & 187 & 75 & 17,300 & 1,230 & 71 & 19,800 & 1,410 & 71 \\
\hline North Carolina .................. & 2,410 & 24 & 169 & 0 & 169 & 70 & 7,640 & 534 & 70 & 10,000 & 703 & 70 \\
\hline North Dakota..................... & 46.4 & 6 & 3.69 & 0 & 3.69 & 80 & 711 & 56.8 & 80 & 757 & 60.5 & 80 \\
\hline Ohio................................... & 1,860 & 16 & 137 & 2.72 & 139 & 75 & 9,750 & 589 & 60 & 11,600 & 729 & 63 \\
\hline 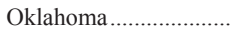 & 357 & 9 & 30.3 & 0 & 30.3 & 85 & 3,550 & 247 & 69 & 3,910 & 277 & 71 \\
\hline Oregon.............................. & 632 & 16 & 66.4 & 7.54 & 73.9 & 117 & 3,400 & 355 & 105 & 4,030 & 429 & 107 \\
\hline Pennsylvania ..................... & 3,470 & 27 & 208 & 0 & 208 & 60 & 9,330 & 522 & 56 & 12,800 & 731 & 57 \\
\hline Rhode Island ..................... & 113 & 11 & 6.57 & 0 & 6.57 & 58 & 943 & 56.0 & 59 & 1,060 & 62.6 & 59 \\
\hline South Carolina .................. & 1,180 & 24 & 118 & 0 & 118 & 100 & 3,710 & 371 & 100 & 4,900 & 490 & 100 \\
\hline South Dakota..................... & 129 & 15 & 5.56 & 0 & 5.56 & 43 & 752 & 43.7 & 58 & 881 & 49.3 & 56 \\
\hline 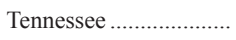 & 594 & 9 & 42.8 & 0 & 42.8 & 72 & 6,010 & 486 & 81 & 6,600 & 529 & 80 \\
\hline Texas .................................... & 1,320 & 5 & 137 & 0 & 137 & 104 & 26,200 & 2,120 & 81 & 27,500 & 2,260 & 82 \\
\hline Utah & 61.4 & 2 & 10.4 & 0 & 10.4 & 169 & 2,930 & 496 & 169 & 3,000 & 506 & 169 \\
\hline Vermont.............................. & 244 & 39 & 11.0 & 0 & 11.0 & 45 & 382 & 16.6 & 43 & 626 & 27.5 & 44 \\
\hline Virginia................................. & 1,560 & 19 & 125 & 0 & 125 & 80 & 6,820 & 546 & 80 & 8,380 & 671 & 80 \\
\hline 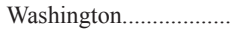 & 1,020 & 14 & 110 & 0 & 110 & 107 & 6,150 & 631 & 103 & 7,170 & 741 & 103 \\
\hline West Virginia .................... & 392 & 21 & 31.3 & 0 & 31.3 & 80 & 1,450 & 116 & 80 & 1,840 & 147 & 80 \\
\hline Wisconsin & 1,600 & 28 & 76.4 & 0 & 76.4 & 48 & 4,170 & 240 & 58 & 5,770 & 317 & 55 \\
\hline 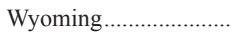 & 119 & 20 & 8.93 & 0 & 8.93 & 75 & 467 & 82.3 & 176 & 586 & 91.3 & 156 \\
\hline Puerto Rico........................ & 4.71 & 0 & 0.52 & 0 & 0.52 & 110 & 3,470 & 338 & 98 & 3,470 & 339 & 98 \\
\hline U.S. Virgin Islands ....... & 51.6 & 50 & 1.23 & 2.55 & 3.78 & 73 & 51.9 & 2.74 & 53 & 104 & 6.52 & 63 \\
\hline TOTAL & 42,500 & 13 & 3,210 & 49.1 & 3,260 & 77 & 283,000 & 23,300 & 82 & 325,000 & 26,600 & 82 \\
\hline
\end{tabular}



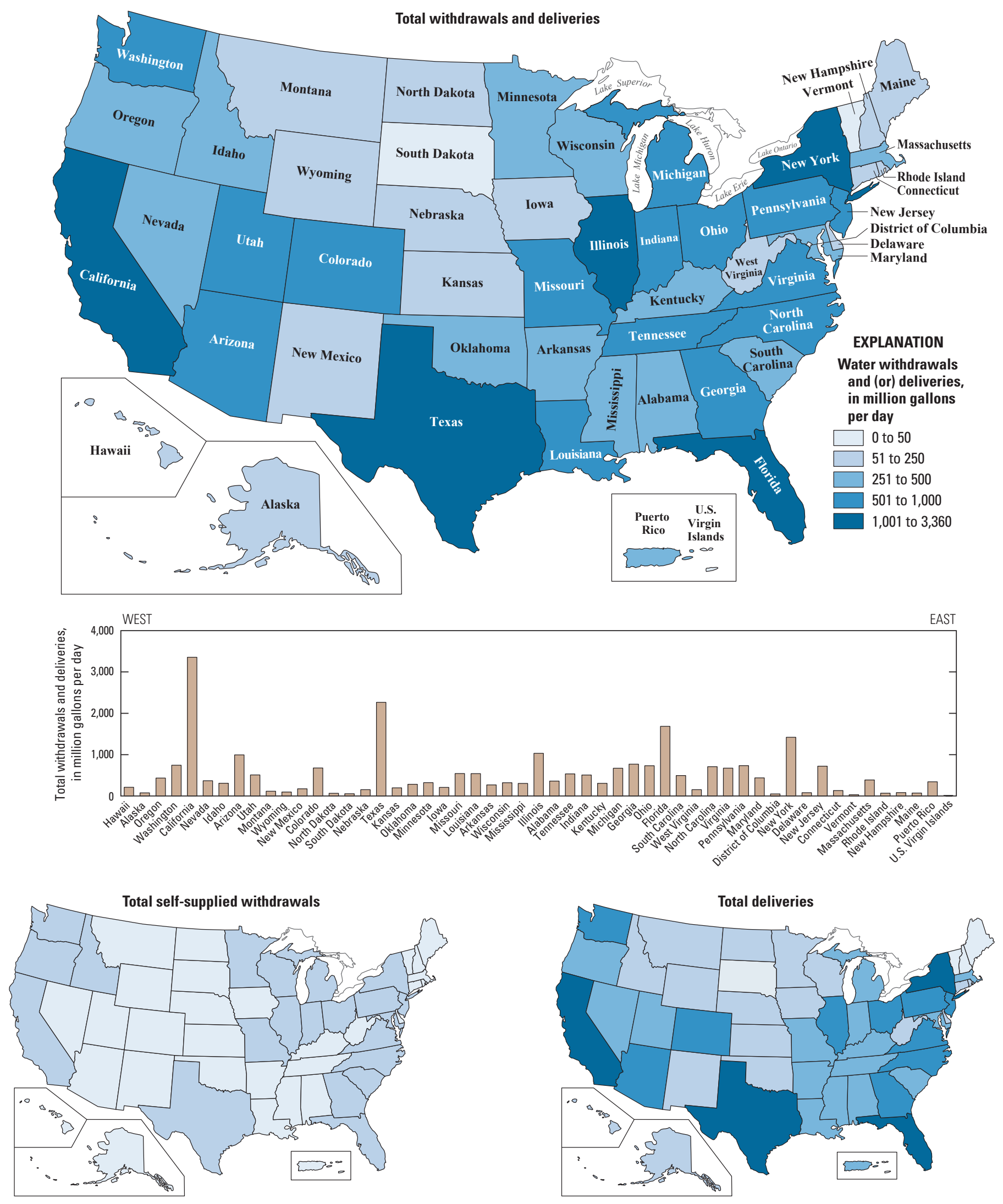

Figure 6A. Domestic withdrawals and deliveries by State, 2015. 

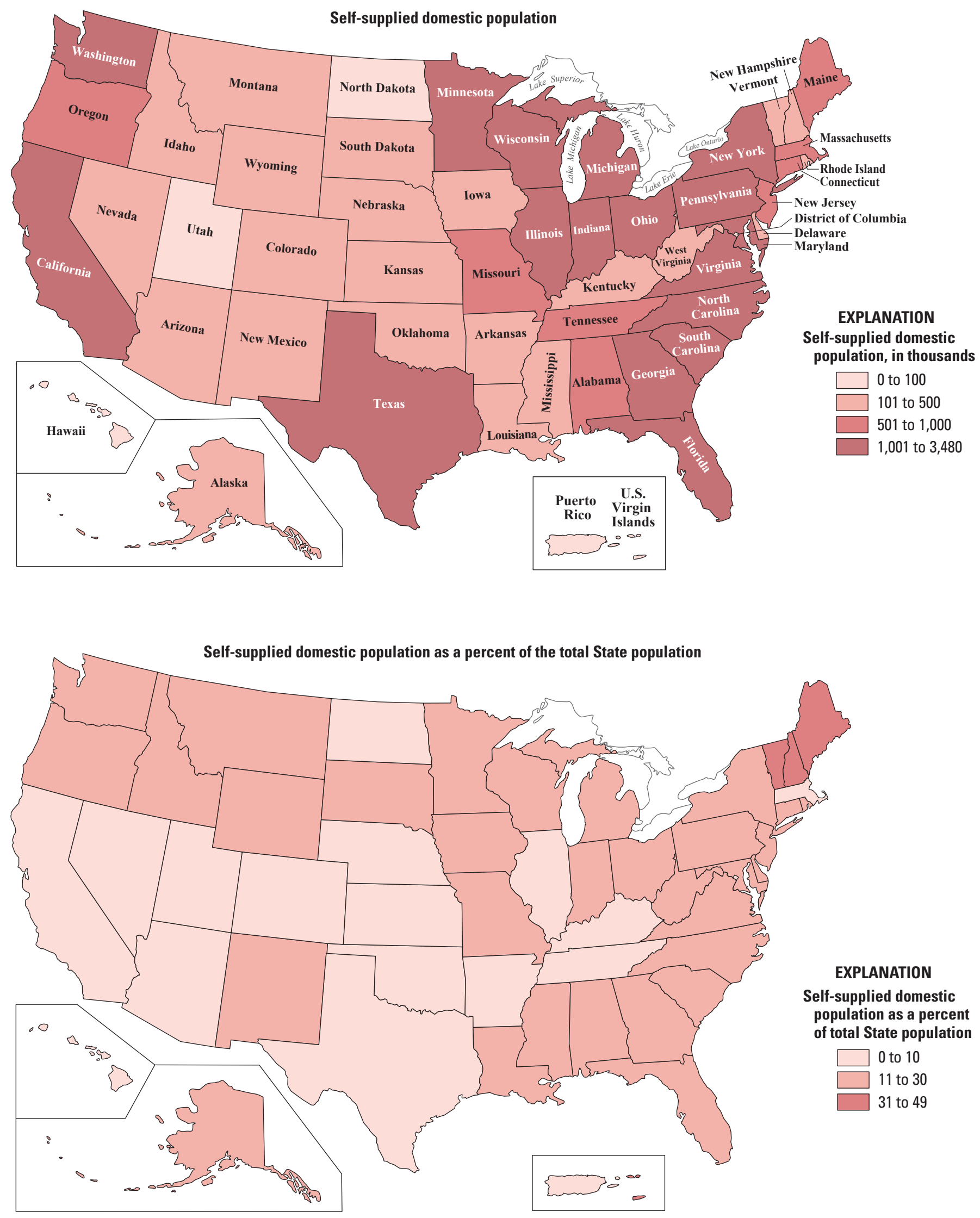

Figure 6B. Self-supplied domestic population and percentage of total population by State, 2015. 
Irrigation water use includes water that is applied by an irrigation system to sustain plant growth in agricultural and horticultural practices. In addition to the irrigation of crops, irrigation of golf courses, parks, nurseries, turf farms, cemeteries, and other self-supplied landscape-watering uses are included in the estimates. Irrigation also includes water that is used for other related processes, including pre-growing season application, frost protection, chemical application, weed control, field preparation, crop cooling, harvesting, dust suppression, and leaching salts from the root zone. Irrigation withdrawals generally are accounted for at the point of diversion (wells, springs, streams, ponds) and include water that was originally withdrawn from the source and water that is lost in conveyance prior to application on fields, as well as water that may subsequently return to a surface-water body as runoff after application, water consumed as evapotranspiration (ET) from plants and evaporation on ground surfaces, or water that recharges aquifers as it seeps past the root zone. Irrigation water use includes self-supplied withdrawals and deliveries from irrigation companies or districts, cooperatives, or governmental entities. All irrigation groundwater and surface-water withdrawals are considered freshwater. Included separately in the irrigation category is reclaimed wastewater from nearby treatment facilities or industries that is used as a source of irrigation water. Irrigated acres are reported for three types of irrigation methods: sprinkler, microirrigation, and surface (flood) systems.
Sources of data for irrigation withdrawals and irrigated acres included State and Federal crop reporting programs, irrigation districts, canal companies, incorporated management areas, satellite data depicting 2015 cropland landscapes, and evapotranspiration estimates. Withdrawals were estimated using information on irrigated crop acreages by crop type and specific crop water-consumption coefficients, or irrigationsystem application rates, as well as soil-moisture balance models. Estimation methods varied from one State to the next and sometimes between geographic areas within a State. Estimation methods ideally included adjustments for climate, system efficiencies, conveyance losses, and other irrigation practices, such as pre-growing season irrigation, salt leaching, or frost protection. Other methods for estimating irrigation withdrawals included extrapolation of sample data on crop water-application rates or power-consumption coefficients. Although estimation methods vary by State and within States, irrigation estimates for the Nation provide a cohesive dataset with which to compare agricultural water use spatially and over time.

Irrigation withdrawals, reclaimed wastewater, and irrigated land by type of irrigation system are listed by State in table 7. For 2015, total groundwater and surface-water irrigation withdrawals were $118,000 \mathrm{Mgal} / \mathrm{d}$, or 132,000 thousand acre-ft/yr, which accounted for 42 percent of total freshwater withdrawals and 64 percent of total freshwater withdrawals for all categories, excluding thermoelectric

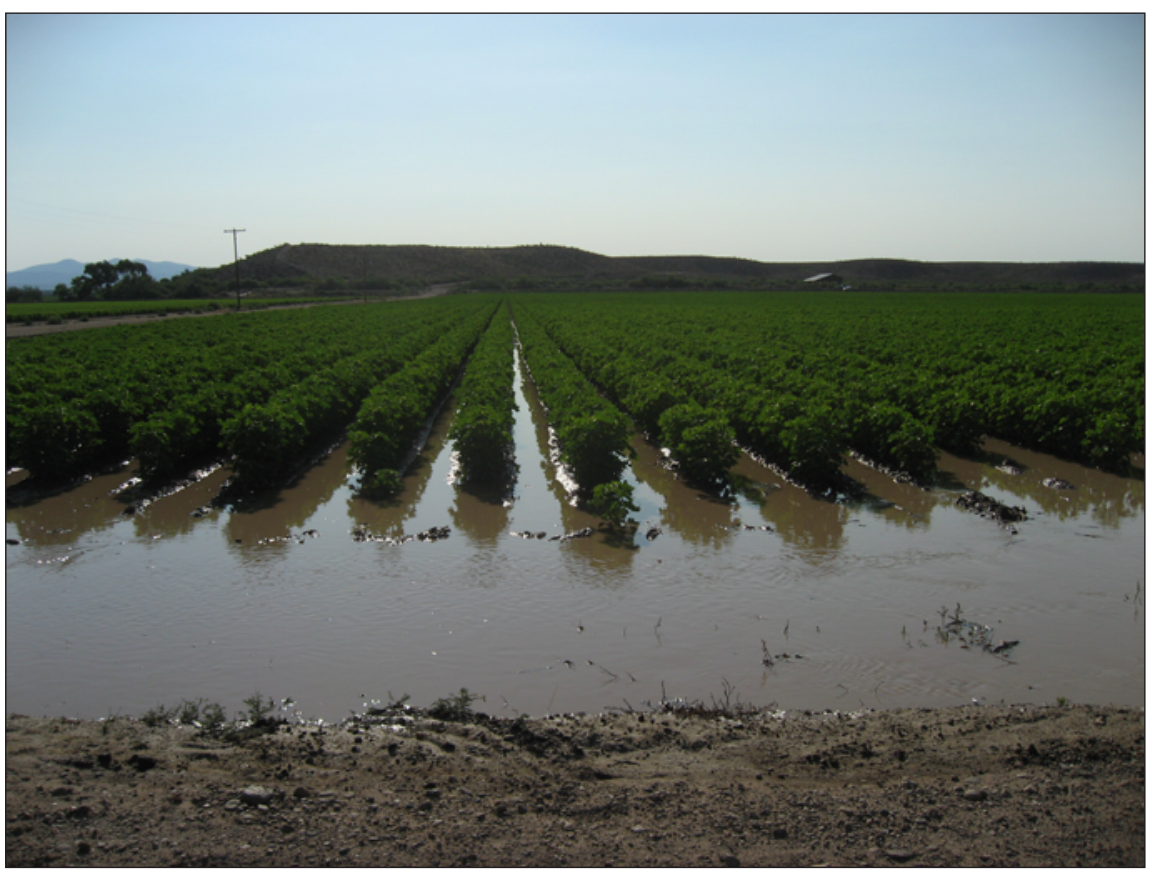

Flood irrigation of cotton, Graham County, Arizona. Photograph by Saeid Tadayon, USGS. 
Table 7. Irrigation water use, 2015.

[Values may not sum to totals because of independent rounding. Abbreviations: Mgal/d, million gallons per day; thousand acre-ft/yr, thousand acre-feet per year; -, not estimated]

\begin{tabular}{|c|c|c|c|c|c|c|c|c|c|c|c|c|c|c|}
\hline \multirow[b]{3}{*}{ State } & \multicolumn{4}{|c|}{$\begin{array}{c}\text { Irrigated land } \\
\text { (thousand acres) }\end{array}$} & \multicolumn{3}{|c|}{$\begin{array}{l}\text { Withdrawals } \\
\text { (Mgal/d) }\end{array}$} & \multirow{3}{*}{$\begin{array}{c}\text { Re- } \\
\text { claimed } \\
\text { waste- } \\
\text { water } \\
\text { (Mgal/d) }\end{array}$} & \multirow{3}{*}{$\begin{array}{c}\text { Con- } \\
\text { sump- } \\
\text { tive use } \\
\text { (Mgal/d) }\end{array}$} & \multicolumn{3}{|c|}{$\begin{array}{c}\text { Withdrawals } \\
\text { (thousand acre-ft/yr) }\end{array}$} & \multirow{3}{*}{$\begin{array}{c}\text { Re- } \\
\text { claimed } \\
\text { waste- } \\
\text { water } \\
\text { (thou- } \\
\text { sand } \\
\text { acre- } \\
\text { ft/yr) }\end{array}$} & \multirow{3}{*}{$\begin{array}{l}\text { Con- } \\
\text { sump- } \\
\text { tive use } \\
\text { (thou- } \\
\text { sand } \\
\text { acre- } \\
\mathrm{ft} / \mathrm{yr} \text { ) }\end{array}$} \\
\hline & \multicolumn{3}{|c|}{ By type of irrigation } & \multirow[b]{2}{*}{ Total } & \multicolumn{2}{|c|}{ By source } & \multirow[b]{2}{*}{ Total } & & & \multicolumn{2}{|c|}{ By source } & & & \\
\hline & Sprinkler & $\begin{array}{l}\text { Micro- } \\
\text { irrigation }\end{array}$ & Surface & & $\begin{array}{l}\text { Ground- } \\
\text { water }\end{array}$ & $\begin{array}{l}\text { Surface } \\
\text { water }\end{array}$ & & & & $\begin{array}{l}\text { Ground- } \\
\text { water }\end{array}$ & $\begin{array}{c}\text { Surface } \\
\text { water }\end{array}$ & Total & & \\
\hline Alabama ………...................... & 189 & 0 & 0 & 189 & 98.8 & 124 & 223 & - & 223 & 111 & 139 & 250 & - & 250 \\
\hline Alaska ................................ & 2.95 & 0 & 0.07 & 3.02 & 1.50 & 0.02 & 1.52 & - & 1.39 & 1.68 & 0.02 & 1.70 & - & 1.56 \\
\hline Arizona & 252 & 57.4 & 730 & 1,040 & 1,970 & 2,560 & 4,530 & 106 & 3,660 & 2,210 & 2,870 & 5,080 & 119 & 4,100 \\
\hline Arkansas............................ & 348 & 0 & 4,080 & 4,430 & 9,280 & 2,290 & 11,600 & 0 & 7,580 & 10,400 & 2,560 & 13,000 & 0 & 8,500 \\
\hline California & 1,690 & 3,530 & 4,110 & 9,320 & 13,900 & 5,130 & 19,000 & 289 & 14,700 & 15,500 & 5,750 & 21,300 & 324 & 16,500 \\
\hline Colorado ………….............. & 1,430 & 2.70 & 1,600 & 3,040 & 1,310 & 7,690 & 9,000 & 4.28 & 2,650 & 1,460 & 8,620 & 10,100 & 4.80 & 2,970 \\
\hline Connecticut ....................... & 17.5 & 0.71 & 0 & 18.2 & 3.02 & 8.29 & 11.3 & - & 10.7 & 3.39 & 9.29 & 12.7 & - & 12.0 \\
\hline Delaware ............................. & 127 & 1.06 & 0 & 128 & 95.4 & 17.5 & 113 & - & 94.7 & 107 & 19.6 & 127 & - & 106 \\
\hline District of Columbia ... & 0.12 & 0 & 0 & 0.12 & 0 & 0.05 & 0.05 & - & 0.04 & 0 & 0.06 & 0.06 & - & 0.04 \\
\hline Florida ................................ & 590 & 720 & 716 & 2,030 & 1,150 & 1,290 & 2,450 & 195 & 1,700 & 1,290 & 1,450 & 2,740 & 219 & 1,900 \\
\hline Georgia & 1,390 & 55.0 & 0 & 1,450 & 564 & 175 & 738 & - & 738 & 632 & 196 & 827 & - & 827 \\
\hline Hawaii ............................. & 11.6 & 123 & 0 & 135 & 73.8 & 311 & 385 & - & 323 & 82.7 & 349 & 432 & - & 362 \\
\hline Idaho ..................................... & 2,570 & 3.08 & 1,220 & 3,790 & 4,900 & 10,400 & 15,300 & - & 8,920 & 5,490 & 11,600 & 17,100 & - & 10,000 \\
\hline 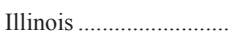 & 625 & 0 & 0 & 625 & 219 & 14.6 & 234 & 1.62 & 219 & 245 & 16.3 & 262 & 1.82 & 245 \\
\hline Indiana................................ & 449 & 0 & 0 & 449 & 93.1 & 40.0 & 133 & - & 120 & 104 & 44.8 & 149 & - & 134 \\
\hline Iowa................................... & 165 & 0 & 0 & 165 & 32.2 & 2.84 & 35.0 & - & 28.1 & 36.1 & 3.18 & 39.2 & - & 31.5 \\
\hline 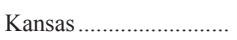 & 2,990 & 39.8 & 92.7 & 3,120 & 2,560 & 122 & 2,680 & 3.94 & 2,200 & 2,870 & 136 & 3,000 & 4.42 & 2,460 \\
\hline 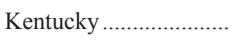 & 68.4 & 10.3 & 0.63 & 79.3 & 2.84 & 36.7 & 39.6 & - & 35.7 & 3.18 & 41.2 & 44.3 & - & 40.0 \\
\hline Louisiana ........................ & 90.2 & 0 & 881 & 972 & 720 & 333 & 1,050 & - & 700 & 807 & 373 & 1,180 & - & 784 \\
\hline Maine .............................. & 40.0 & 0.05 & 0.81 & 40.9 & 5.06 & 13.8 & 18.9 & - & 18.0 & 5.67 & 15.5 & 21.2 & - & 20.2 \\
\hline Maryland ......................... & 110 & 8.95 & 0.68 & 120 & 48.3 & 15.8 & 64.1 & - & 56.9 & 54.1 & 17.7 & 71.8 & - & 63.8 \\
\hline Massachusetts ................. & 25.7 & 2.06 & 12.7 & 40.5 & 114 & 24.1 & 139 & - & 29.8 & 128 & 27.0 & 155 & - & 33.4 \\
\hline 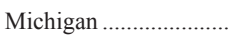 & 563 & 32.7 & 1.68 & 598 & 261 & 70.6 & 332 & - & 299 & 293 & 79.2 & 372 & - & 335 \\
\hline Minnesota........................... & 667 & 0 & 26.5 & 694 & 242 & 33.9 & 276 & 0 & 218 & 272 & 38.0 & 310 & 0 & 245 \\
\hline Mississippi ...................... & 393 & 0 & 1,260 & 1,650 & 1,640 & 130 & 1,770 & - & 1,350 & 1,840 & 145 & 1,980 & - & 1,510 \\
\hline 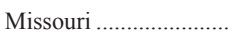 & 492 & 53.6 & 635 & 1,180 & 1,300 & 75.3 & 1,370 & - & 436 & 1,460 & 84.4 & 1,540 & - & 489 \\
\hline Montana ……...................... & 1,280 & 0.64 & 1,190 & 2,470 & 59.6 & 9,390 & 9,450 & - & 2,370 & 66.8 & 10,500 & 10,600 & - & 2,660 \\
\hline Nebraska ............................ & 7,350 & 16.6 & 2,210 & 9,580 & 5,420 & 674 & 6,090 & - & 5,540 & 6,070 & 755 & 6,830 & - & 6,210 \\
\hline Nevada …………............... & 365 & 0.18 & 332 & 698 & 972 & 1,090 & 2,070 & 5.75 & 1,560 & 1,090 & 1,230 & 2,320 & 6.45 & 1,750 \\
\hline New Hampshire ............. & 6.46 & 0.84 & 0.25 & 7.55 & 1.09 & 4.11 & 5.20 & - & 4.94 & 1.22 & 4.61 & 5.83 & - & 5.54 \\
\hline 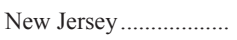 & 81.0 & 31.0 & 2.98 & 115 & 55.1 & 38.8 & 93.9 & - & 63.9 & 61.8 & 43.5 & 105 & - & 71.6 \\
\hline New Mexico....................... & 385 & 18.8 & 355 & 759 & 1,050 & 1,320 & 2,370 & 4.43 & 1,410 & 1,180 & 1,490 & 2,660 & 4.97 & 1,580 \\
\hline 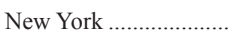 & 84.9 & 15.6 & 1.07 & 102 & 17.8 & 35.7 & 53.5 & - & 51.0 & 20.0 & 40.0 & 60.0 & - & 57.1 \\
\hline North Carolina ................. & 222 & 6.39 & 0 & 228 & 83.8 & 241 & 325 & - & 325 & 93.9 & 270 & 364 & - & 364 \\
\hline North Dakota..................... & 226 & 0 & 52.1 & 278 & 102 & 130 & 233 & 0 & 81.3 & 115 & 146 & 261 & 0 & 91.2 \\
\hline Ohio & 66.9 & 7.70 & 0 & 74.6 & 17.9 & 37.1 & 55.0 & 0 & 49.4 & 20.0 & 41.6 & 61.6 & 0 & 55.3 \\
\hline 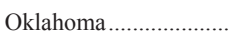 & 471 & 23.9 & 58.0 & 553 & 787 & 144 & 931 & 0 & 761 & 882 & 161 & 1,040 & 0 & 853 \\
\hline Oregon............................. & 1,350 & 88.3 & 520 & 1,960 & 1,220 & 3,940 & 5,160 & - & 3,440 & 1,360 & 4,420 & 5,780 & - & 3,860 \\
\hline Pennsylvania ..................... & 58.0 & 19.5 & 0.44 & 78.0 & 17.5 & 16.8 & 34.3 & - & 31.0 & 19.6 & 18.9 & 38.5 & - & 34.8 \\
\hline Rhode Island ................... & 5.69 & 0.83 & 0.02 & 6.54 & 3.78 & 0.47 & 4.25 & - & 4.04 & 4.24 & 0.53 & 4.76 & - & 4.53 \\
\hline South Carolina ................. & 176 & 10.9 & 6.03 & 193 & 87.9 & 38.3 & 126 & 0 & 126 & 98.5 & 43.0 & 141 & 0 & 141 \\
\hline South Dakota................... & 213 & 0 & 59.3 & 273 & 139 & 71.2 & 211 & 0 & 110 & 156 & 79.8 & 236 & 0 & 124 \\
\hline Tennessee ........................ & 124 & 10.3 & 15.9 & 150 & 36.4 & 27.4 & 63.8 & - & 51.1 & 40.8 & 30.7 & 71.6 & - & 57.3 \\
\hline Texas ............................ & 4,150 & 468 & 1,040 & 5,660 & 4,480 & 1,010 & 5,490 & 45.3 & 4,280 & 5,020 & 1,140 & 6,150 & 50.8 & 4,800 \\
\hline Utah...................................... & 609 & 0.94 & 689 & 1,300 & 537 & 2,490 & 3,030 & 13.0 & 2,060 & 602 & 2,790 & 3,390 & 14.6 & 2,310 \\
\hline Vermont.............................. & 4.68 & 1.47 & 0.65 & 6.80 & 1.02 & 2.09 & 3.11 & - & 2.93 & 1.14 & 2.34 & 3.49 & - & 3.28 \\
\hline 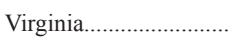 & 95.5 & 11.1 & 0 & 107 & 7.93 & 43.8 & 51.7 & - & 44.0 & 8.89 & 49.0 & 57.9 & - & 49.3 \\
\hline 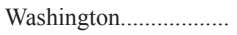 & 1,260 & 76.4 & 223 & 1,560 & 720 & 1,800 & 2,520 & 0 & 1,990 & 807 & 2,020 & 2,830 & 0 & 2,230 \\
\hline West Virginia.................... & 4.14 & 0 & 1.67 & 5.81 & 0.40 & 3.75 & 4.15 & - & 3.52 & 0.45 & 4.20 & 4.65 & - & 3.95 \\
\hline 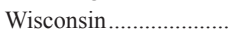 & 508 & 6.09 & 20.0 & 534 & 287 & 173 & 460 & - & 293 & 322 & 194 & 515 & - & 329 \\
\hline Wyoming ............................ & 237 & 5.56 & 1,190 & 1,440 & 537 & 7,250 & 7,790 & - & 2,150 & 602 & 8,130 & 8,730 & - & 2,410 \\
\hline Puerto Rico....................... & 15.6 & 27.9 & 0.77 & 44.2 & 41.6 & 34.1 & 75.7 & - & 73.2 & 46.6 & 38.2 & 84.8 & - & 82.0 \\
\hline U.S. Virgin Islands ....... & 0 & 0 & 0 & 0 & 0 & 0 & 0 & - & 0 & 0 & 0 & 0 & - & 0 \\
\hline TOTAL & 34,700 & 5,490 & 23,300 & 63,500 & 57,200 & 60,900 & 118,000 & 669 & 73,200 & 64,100 & 68,300 & 132,000 & 750 & 82,000 \\
\hline
\end{tabular}




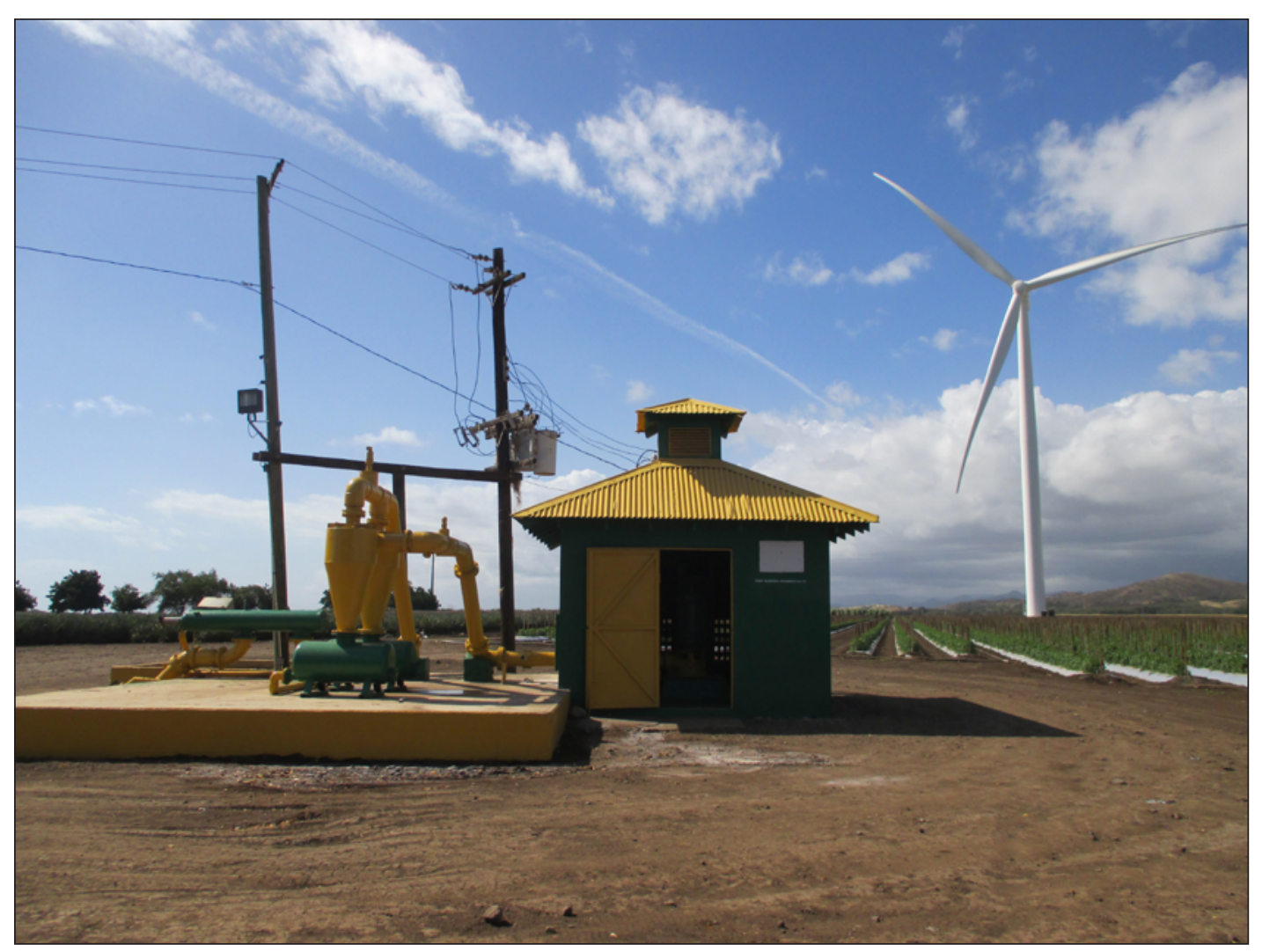

Irrigation well in Puerto Rico. Photograph by José A. Santiago-Saez, USGS.

power. Total irrigation withdrawals were about 2 percent more than in 2010. Withdrawals from surface-water sources were $60,900 \mathrm{Mgal} / \mathrm{d}$, which accounted for 52 percent of the total irrigation withdrawals, and were about 8 percent less than in 2010. Groundwater withdrawals for 2015 were $57,200 \mathrm{Mgal} / \mathrm{d}$, which accounts for 48 percent of the total irrigation withdrawals, and were 16 percent more than in 2010. About 63,500 thousand acres were irrigated in 2015, an increase of 1,130 thousand acres (2 percent) from 2010 . About 34,700 thousand acres (55 percent) were irrigated with sprinkler systems, 23,300 thousand acres with surface (flood), and 5,490 thousand acres with microirrigation systems.

In addition to irrigation withdrawals from groundwater and surface-water sources, the use of reclaimed wastewater as a source of irrigation water was reported in 10 States (California, Florida, Arizona, Texas, Utah, Nevada, New Mexico, Colorado, Kansas, and Illinois) and accounts for $669 \mathrm{Mgal} / \mathrm{d}$ or less than 1 percent of the total irrigation water used. California, Florida, and Arizona were the largest users of reclaimed wastewater for irrigation with uses of 289, 195, and $106 \mathrm{Mgal} / \mathrm{d}$, respectively. Although still a minor overall source of irrigation water in the Nation, reclaimed wastewater use increased from $472 \mathrm{Mgal} / \mathrm{d}$ in 2010 to $669 \mathrm{Mgal} / \mathrm{d}$ in 2015 (a 42-percent increase).
Five states (California, Idaho, Arkansas, Montana, and Colorado) accounted for 54 percent of the national total of irrigation withdrawals. The geographic distribution of total groundwater and surface-water withdrawals for irrigation is shown in figure 7. Most of the total irrigation withdrawals (81 percent) in the United States and irrigated acres ( 74 percent) were in the 17 conterminous Western States (west of the solid line in fig. 7). The 17 Western States cumulatively accounted for 91 percent of total surface-water irrigation withdrawals and 71 percent of total groundwater irrigation withdrawals in the Nation. Additionally, 9 of the 10 States with the highest irrigation withdrawals are Western States. Agricultural regions in the conterminous Western States are typically in areas where average annual precipitation is less than 20 inches, which is generally insufficient to support crops without supplemental water. Surface water accounted for 58 percent of the total withdrawals in the arid West, but groundwater was the dominant source of irrigation water in California, Nebraska, Texas, Kansas, South Dakota, and Oklahoma. Irrigation water is typically applied in the more humid conterminous Eastern States (east of the solid line in fig. 7), as well as in Hawaii and Puerto Rico, to supplement precipitation during critical periods of the growing season, increase yields of crops, and increase the number of plantings 

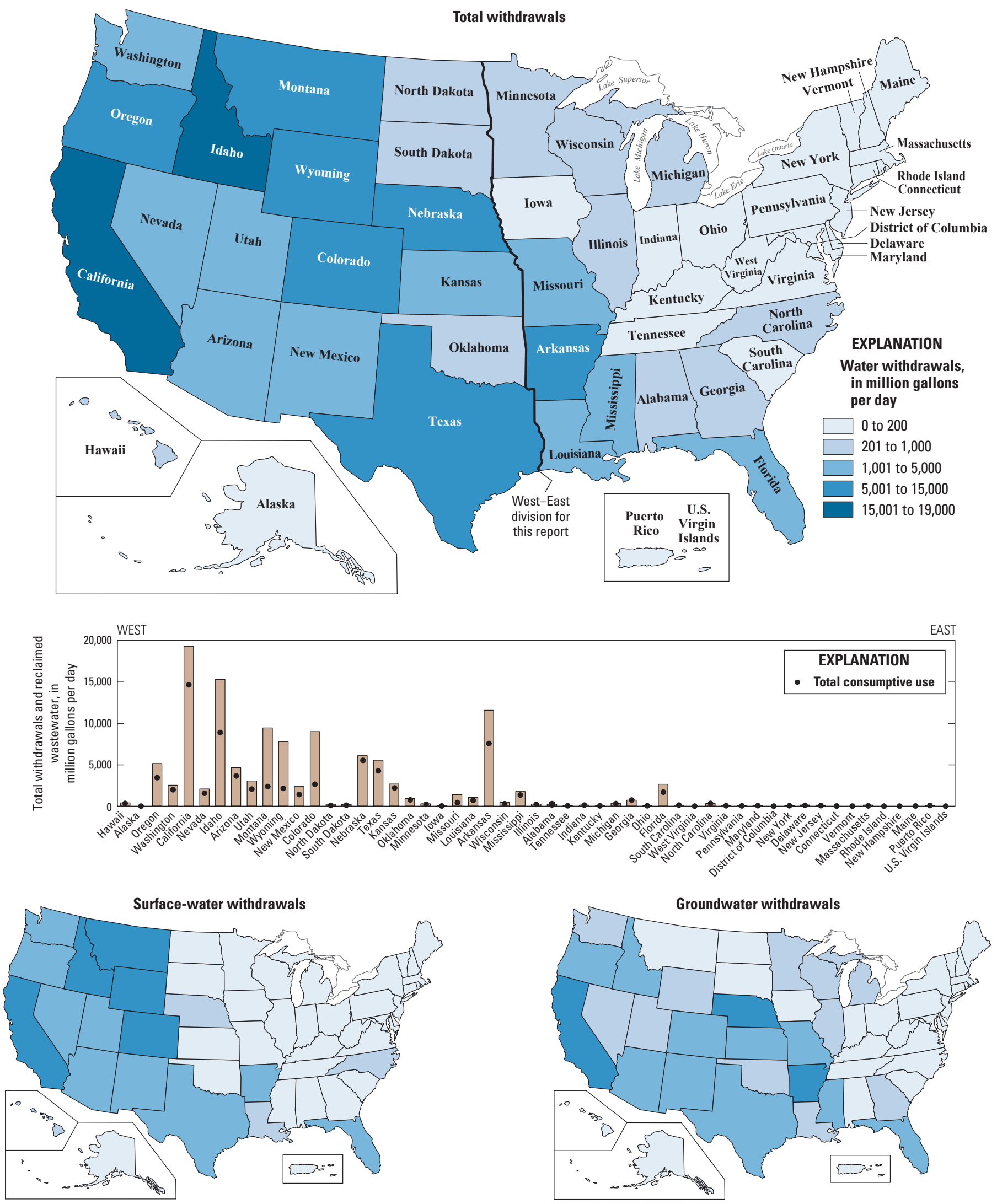

Figure 7. Irrigation water use by source and State, 2015. 
per year. Notable Eastern States include Arkansas with the third largest withdrawals $(11,600 \mathrm{Mgal} / \mathrm{d})$ and fourth largest number of irrigated acres $(4,430)$ and Florida with the fourteenth largest irrigation withdrawals $(2,450 \mathrm{Mgal} / \mathrm{d})$ and ninth largest number of irrigated acres $(2,030)$.

Average application rates are calculated as a function of total irrigation withdrawals and total irrigated acres. The national application rate (total withdrawals for irrigation divided by the total acres irrigated) for 2015 was 2.09 acre-feet per acre, which is near that of the 2010 average of 2.08 acrefeet per acre. The median State application rate is 0.94 acrefeet per acre. The highest application rates occur in the arid Western States (Wyoming, Arizona, Idaho, and Montana had the highest application rates with values of $6.08,4.89$, and 4.52 , and 4.29 acre-feet per acre, respectively) in order to meet the crop water requirements in these areas with limited precipitation. Many Western States rely on surface water as a primary source of irrigation water, and surface (flood) irrigation systems are common, which ultimately results in higher application rates. Additionally, surface water is often conveyed in canals and ditches and can lose substantial amounts of water via evaporation and infiltration before the remaining water reaches the irrigation systems and is applied to the land surface. Transmission losses are likely minimal for groundwater as water is commonly transferred from a well through pipes to an irrigation system. In addition, 33 percent of irrigated lands in the Western States is irrigated with relatively inefficient surface (flood) irrigation. Considerable amounts of excess applied water may result in return flow (surface runoff that reaches a surface-water source or infiltrates past the root zone to potentially recharge the groundwater system), thus returning to the local hydrologic system.

Although national total irrigation withdrawals were similar in 2010 and 2015, notable changes were observed at the State level. Of the States with the largest total irrigation withdrawals (greater than 5,000 Mgal/d), Arkansas, Montana, and Wyoming had notable increases of irrigation withdrawals, and Texas and California had notable decreases. Arkansas irrigation withdrawals increased 2,850 Mgal/d (33 percent) from 2010 to 2015 , although total irrigated acres decreased 5 percent. Arkansas application rates increased from 2.09 acre-ft/acre in 2010 to 2.93 acre-ft/acre in 2015. Montana irrigation withdrawals increased 2,070 Mgal/d (28 percent) from 2010 to 2015, which corresponds with a 48 percent increase in estimated total irrigated acres during the same period. Because total irrigation withdrawals and total irrigated acres increased, application rates in Montana decreased from 4.95 acre-ft/acre in 2010 to 4.29 acre-ft/acre in 2015. Wyoming irrigation withdrawals increased $3,420 \mathrm{Mgal} / \mathrm{d}$ (78 percent), and irrigated acres increased 33 percent from 2010 to 2015. Despite the increase in irrigated acres, Wyoming application rates increased from 4.53 acre-ft/acre in 2010 to 6.08 acre-ft/acre in 2015. Texas irrigation withdrawals decreased 1,340 Mgal/d (20 percent), and irrigated acres decreased 4 percent from 2010 to 2015 . Texas was nearing the end of a multiyear drought in 2015 and experienced very moist conditions in June 2015 (fig. 8A) and extreme drought conditions in September 2015 (fig. 8B) (National Oceanic and Atmospheric Administration, 2016b). Texas application rates decreased from 1.29 acre-ft/acre in 2010 to 1.09 acre-ft/acre in 2015. California irrigation withdrawals declined $4,070 \mathrm{Mgal} / \mathrm{d}$ (18 percent), and irrigated acres decreased by 10 percent from 2010, likely as a result of the intense drought conditions in the region in 2015 (fig. 8A). Historically (1950-2010), surface water has been the primary source of irrigation water in California. However, groundwater was the primary source of irrigation water in California in 2015, likely as a result of limited available surface-water resources during the period of intense drought (fig. 8A) (National Oceanic and Atmospheric Administration, 2016b).

Nationwide estimates of consumptive use of water withdrawn for irrigation are included in this report for the first time since 1995. Consumptive use of irrigation water represents the fraction of water that was originally withdrawn from a source for irrigation and is subsequently removed from availability owing to evaporation, transpiration, or incorporation into crops. Reliable estimates of consumptive use are oftentimes difficult to determine. In most States, consumptive use was based on coefficients, irrigation-system efficiencies, or theoretical crop requirements from various sources. The NWUSP provided USGS personnel with evapotranspiration estimates based on 1-kilometer scale MODIS satellite data that were analyzed through the SSEBop model (Senay and others, 2013) to aid in interpretation of consumptive use estimates on irrigated lands. Consumptive-use estimates in California, Colorado, Delaware, Kansas, Missouri, Montana, North Dakota, South Dakota, and Wyoming were based primarily on the SSEBop model data; however, other methods were used to estimate consumptive use for the other States.

Of the $118,000 \mathrm{Mgal} / \mathrm{d}$ of groundwater and surface water withdrawn and the $669 \mathrm{Mgal} / \mathrm{d}$ of reclaimed wastewater used in the United States for irrigation in 2015, it is estimated that $73,200 \mathrm{Mgal} / \mathrm{d}$ (62 percent of the total water used) was consumed. State estimates of consumptive use range from 22 percent to 100 percent of the withdrawn irrigation water and reclaimed wastewater used for irrigation. Four States (Alabama, Georgia, North Carolina, and South Carolina) assumed 100 percent consumptive use where consumptive use was set equal to the water withdrawn for irrigation. The four States with the lowest percentage of estimated consumptive use (less than 30 percent) include Massachusetts, Montana, Wyoming, and Colorado; therefore, it is estimated that less than 30 percent of the water originally used for irrigation was actually evaporated, evapotranspired, or incorporated into the irrigated crops in those States. 
A

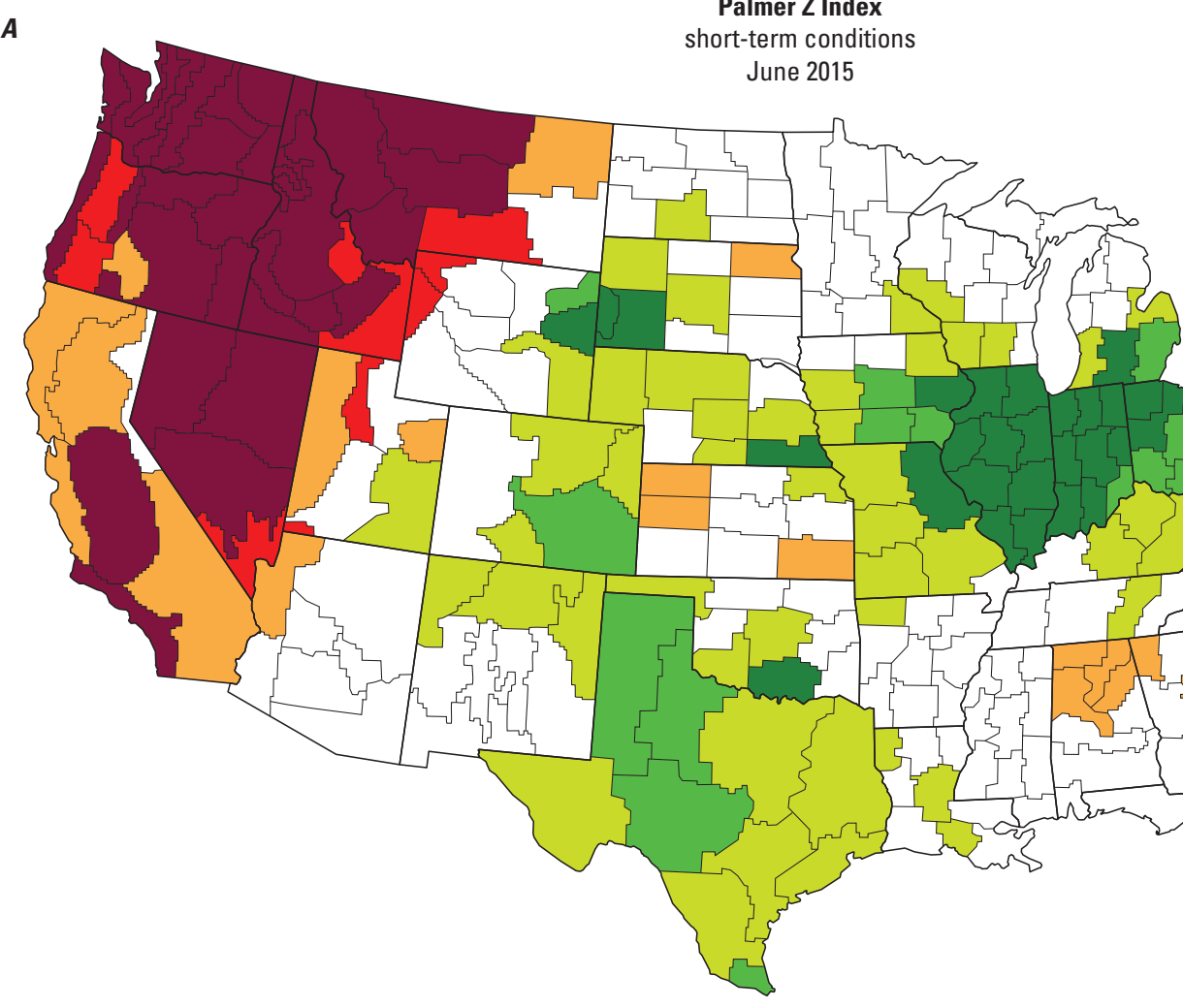

Palmer Z Index

ort-term conditions

EXPLANATION

Extreme drought -2.75 and below Severe drought -2.00 to -2.74 Moderate drought -1.25 to -1.99 Mid-range -1.24 to 0.99 Moderately moist 1.00 to 2.49 Very moist 2.50 to 3.49 Extremely moist 3.50 and above

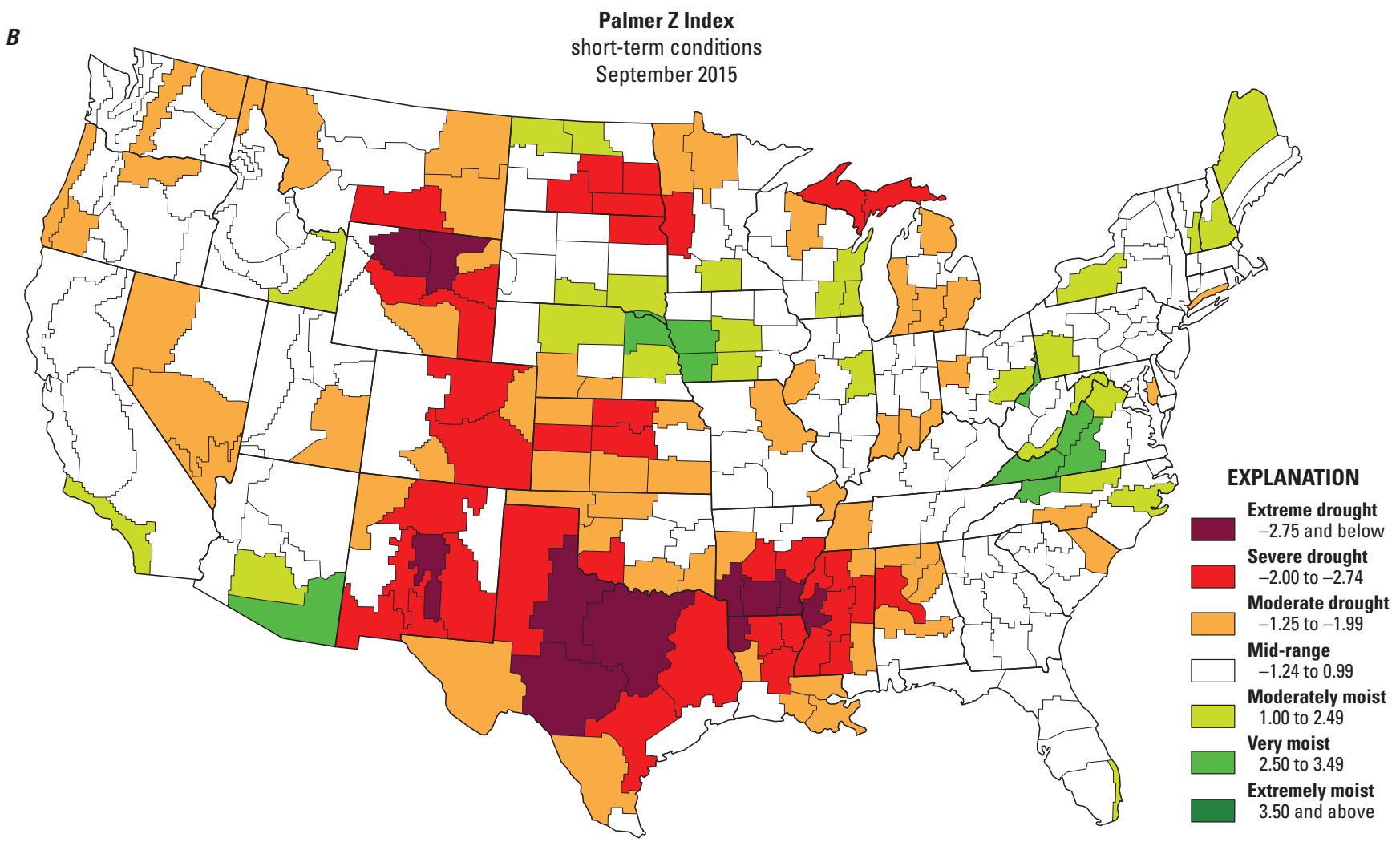

B

Figure 8. Drought index maps for the conterminous United States for $A$, June and $B$, September 2015. [Original map does not include Hawaii, Puerto Rico, or U.S. Virgin Islands. From National Oceanic and Atmospheric Administration, 2016b.] 
Livestock water use is water associated with livestock watering, feedlots, dairy operations, and other on-farm needs. Livestock includes dairy cows and heifers, beef cattle and calves, sheep and lambs, goats, hogs and pigs, horses, and poultry. Other livestock water uses include cooling of facilities for the animals and products, dairy sanitation and wash down of facilities, animal waste-disposal systems, and incidental water losses. All withdrawals were considered freshwater and self-supplied. The livestock category excludes on-farm domestic use, lawn and garden watering, and irrigation water use.

Few State agencies require livestock operations to report water withdrawals; therefore, most estimates of livestock withdrawals were derived using animal population data and water-use coefficients, in gallons per head per day for each animal type. Animal population data generally are available from State agricultural agencies and the USDA NASS. Coefficients vary by State and, for many States, were provided by agricultural extension agents or water-permitting agencies. Coefficients may reflect facility maintenance needs and effects of climate on animal watering. Many of the 2015 withdrawals for livestock were estimated according to methods described by Lovelace (2009a), using livestock population data compiled for the USDA NASS 2012 Census of Agriculture (U.S. Department of Agriculture, 2014a) and water-use coefficients.

Livestock withdrawals for 2015 are listed by State in table 8. During 2015, withdrawals for livestock use were an estimated 2,000 Mgal/d, or 2,240 thousand acre-ft/yr (table 2B). Livestock withdrawals were about less than 1 percent of total freshwater withdrawals and about 1 percent of total freshwater withdrawals for all categories, excluding thermoelectric power. Groundwater was the source for 62 percent of total livestock withdrawals. Estimated total livestock withdrawals for 2015 were the same as 2010 .

The geographic distribution of total, surface-water, and groundwater livestock withdrawals is shown in figure 9. Texas, California, Iowa, Nebraska, and Kansas each used more than $100 \mathrm{Mgal} / \mathrm{d}$ for livestock and together accounted for 42 percent of total livestock withdrawals in 2015. Texas, Iowa, Nebraska, Kansas, and California each used more than $80 \mathrm{Mgal} / \mathrm{d}$ of groundwater for livestock (table 8) and accounted for 42 percent of groundwater withdrawals for this use. Texas and California each used more than $100 \mathrm{Mgal} / \mathrm{d}$ of surface water for livestock, and accounted for 31 percent of surface-water withdrawals for livestock. It is likely that a large portion of the withdrawals for livestock was used for cattle, which typically require more water than other livestock species, with the exception of horses, and milk cows require additional water for sanitation of milking facilities and equipment (Lovelace, 2009a). In 2012, Texas, California, Iowa, Nebraska, and Kansas together accounted for 36 percent of all cattle and calves and 29 percent of all milk cows in the United States (U.S. Department of Agriculture, 2014a).
Table 8. Livestock water withdrawals, 2015.

[Values may not sum to totals because of independent rounding]

\begin{tabular}{|c|c|c|c|}
\hline \multirow{3}{*}{ State } & \multicolumn{3}{|c|}{$\begin{array}{c}\text { Withdrawals } \\
\text { (million gallons per day) }\end{array}$} \\
\hline & \multicolumn{2}{|c|}{ By source } & \multirow{2}{*}{ Total } \\
\hline & Groundwater & Surface water & \\
\hline Alabama ................................ & 11.5 & 14.7 & 26.2 \\
\hline Alaska ................................ & 0.04 & 0.09 & 0.13 \\
\hline Arizona & 38.9 & 0 & 38.9 \\
\hline Arkansas............................. & 13.6 & 20.5 & 34.1 \\
\hline California ............................ & 82.0 & 101 & 183 \\
\hline Colorado............................. & 22.7 & 10.6 & 33.3 \\
\hline Connecticut ........................ & 1.15 & 0 & 1.15 \\
\hline Delaware ............................. & 1.34 & 0 & 1.34 \\
\hline District of Columbia ... & 0 & 0 & 0 \\
\hline Florida & 25.7 & 0.35 & 26.1 \\
\hline 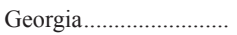 & 27.5 & 17.4 & 44.9 \\
\hline Hawaii ................................. & 0.51 & 1.10 & 1.61 \\
\hline Idaho ................................. & 41.2 & 9.57 & 50.8 \\
\hline Illinois ............................... & 36.2 & 0 & 36.2 \\
\hline Indiana................................... & 26.2 & 13.0 & 39.2 \\
\hline Iowa..................................... & 124 & 41.0 & 165 \\
\hline Kansas ................................ & 83.5 & 20.4 & 104 \\
\hline Kentucky ........................... & 2.07 & 38.7 & 40.8 \\
\hline Louisiana & 3.16 & 3.19 & 6.35 \\
\hline Maine ….............................. & 1.54 & 0.51 & 2.05 \\
\hline Maryland ............................ & 5.85 & 2.15 & 8.00 \\
\hline Massachusetts ................ & 0.64 & 0.39 & 1.03 \\
\hline Michigan ............................ & 21.4 & 2.33 & 23.7 \\
\hline Minnesota............................ & 58.9 & 0 & 58.9 \\
\hline 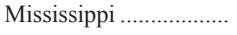 & 6.85 & 10.3 & 17.1 \\
\hline Missouri ............................ & 16.1 & 47.6 & 63.7 \\
\hline Montana ……...................... & 12.3 & 29.9 & 42.2 \\
\hline Nebraska ............................. & 89.7 & 20.4 & 110 \\
\hline Nevada ……………........... & 4.94 & 0 & 4.94 \\
\hline New Hampshire ............ & 0.63 & 0.21 & 0.84 \\
\hline New Jersey ....................... & 0.88 & 0 & 0.88 \\
\hline New Mexico....................... & 29.8 & 2.25 & 32.0 \\
\hline New York .......................... & 16.8 & 8.89 & 25.7 \\
\hline North Carolina ............... & 51.9 & 14.6 & 66.5 \\
\hline North Dakota...................... & 12.5 & 8.30 & 20.8 \\
\hline Ohio..................................... & 7.83 & 16.8 & 24.6 \\
\hline Oklahoma ............................ & 27.4 & 43.3 & 70.6 \\
\hline Oregon.............................. & 3.02 & 13.2 & 16.3 \\
\hline Pennsylvania ...................... & 36.3 & 3.17 & 39.5 \\
\hline Rhode Island ..................... & 0.11 & 0.01 & 0.12 \\
\hline South Carolina .................. & 4.36 & 5.51 & 9.87 \\
\hline South Dakota..................... & 19.3 & 28.6 & 47.9 \\
\hline Tennessee ........................... & 12.0 & 11.5 & 23.4 \\
\hline Texas ................................. & 138 & 137 & 276 \\
\hline Utah.................................... & 7.46 & 8.44 & 15.9 \\
\hline Vermont................................ & 4.41 & 1.46 & 5.87 \\
\hline 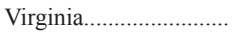 & 6.52 & 20.5 & 27.0 \\
\hline Washington.......................... & 20.8 & 8.82 & 29.7 \\
\hline West Virginia...................... & 1.70 & 3.38 & 5.08 \\
\hline 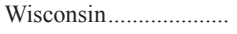 & 67.0 & 7.44 & 74.5 \\
\hline 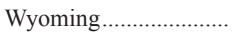 & 6.17 & 10.0 & 16.2 \\
\hline Puerto Rico....................... & 4.23 & 1.60 & 5.83 \\
\hline U.S. Virgin Islands ....... & 0.01 & 0.01 & 0.02 \\
\hline TOTAL & 1,240 & 760 & 2,000 \\
\hline
\end{tabular}



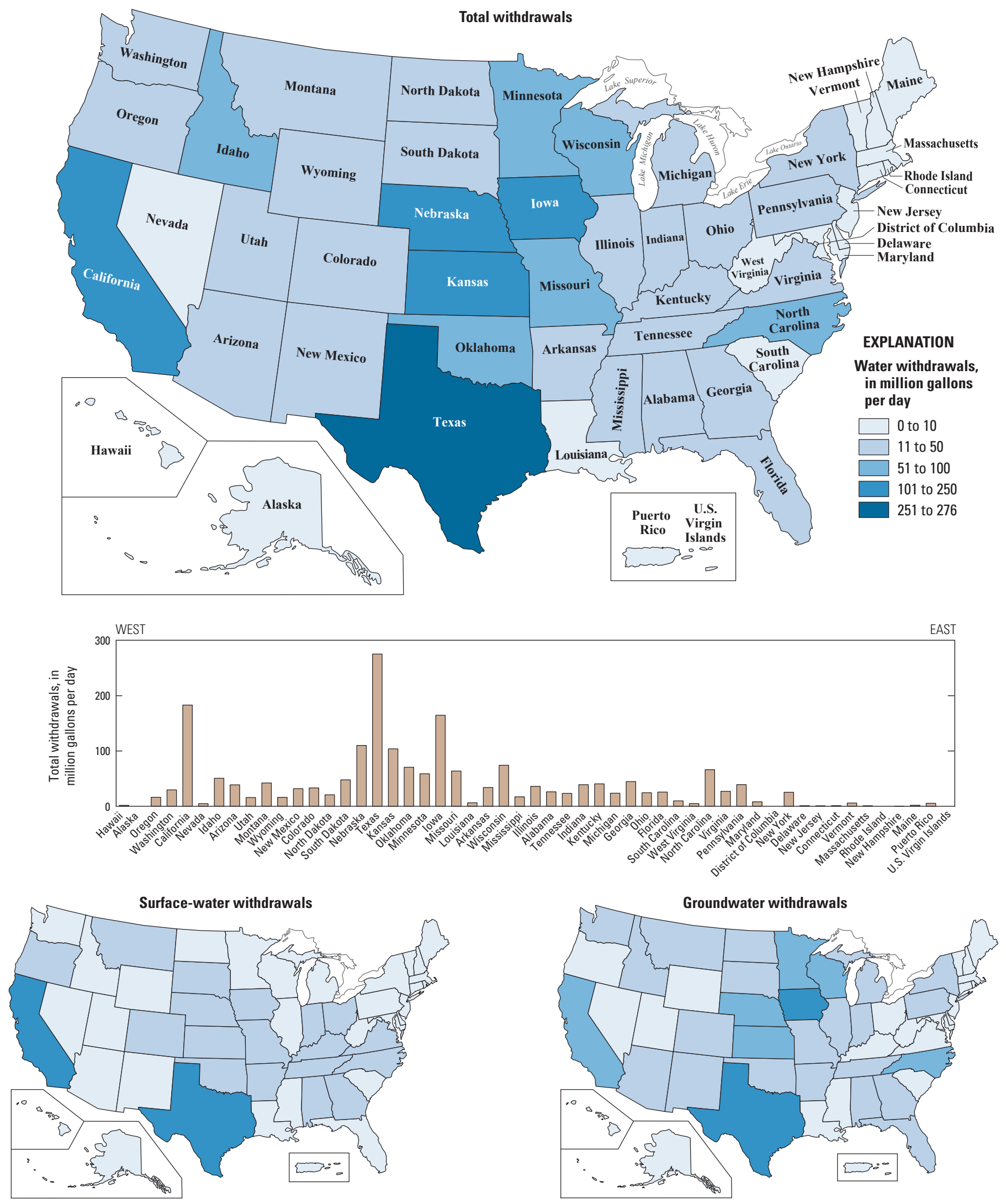

Figure 9. Livestock withdrawals by source and State, 2015. 


\section{Aquaculture}

7,550 million gallons per day

Aquaculture water use is water associated with raising organisms that live in water, such as finfish and shellfish, for food, restoration, conservation, or sport. Aquaculture production occurs under controlled feeding, sanitation, and harvesting procedures primarily in ponds, flow-through raceways, and, to a lesser extent, cages, net pens, and closed-recirculation tanks. All aquaculture withdrawals were considered self-supplied.

Many of the 2015 withdrawals for aquaculture were estimated according to methods described by Lovelace (2009b), using aquaculture data compiled for the USDA NASS 2013 Census of Aquaculture (U.S. Department of Agriculture, 2014b) with standardized water-use coefficients and water-replacement rates. The USDA NASS 2013 data include statistics for various aquacultured species and aquaculture ponds, raceways, tanks, egg incubators, pens, and cages at commercial and noncommercial aquaculture operations. Several other sources of information were also used to estimate 2015 aquaculture withdrawals. These other sources include State permits from which estimates were derived from reported water withdrawals or return flows for aquaculture facilities. The EPA Permit Compliance System database also was a source of return-flow data that were used to estimate water withdrawals. Individual aquaculture operations, State regulatory agencies, State offices of the USDA NASS, and Cooperative Extension Service offices also provided information that was used to estimate aquaculture withdrawals in some States.

Total withdrawals for aquaculture during 2015, $7,550 \mathrm{Mgal} / \mathrm{d}$, or 8,460 thousand acre-ft/yr (table $2 B$ ), are listed by State in table 9. Surface water was the source for about 79 percent of the withdrawals for this category. Much of the surface water was used for flow-through raceways and was returned to the source after use. A combined total of $6.30 \mathrm{Mgal} / \mathrm{d}$ saline surface-water withdrawals, less than 0.1 percent of total aquaculture withdrawals, was reported in Connecticut (3.02 Mgal/d), Florida (2.02 Mgal/d), Texas $(0.86 \mathrm{Mgal} / \mathrm{d})$, and Maine $(0.40 \mathrm{Mgal} / \mathrm{d})$; these amounts are not shown separately in table 9 , but are included in the total. Aquaculture withdrawals were about 2 percent of total withdrawals and about 4 percent of total withdrawals for all categories, excluding thermoelectric power. Estimated aquaculture withdrawals in 2015 were 16 percent less than in 2010 .

The geographic distribution of total, surface-water, and groundwater withdrawals for aquaculture is shown in figure 10. Idaho, North Carolina, California, and Oregon each used more than $500 \mathrm{Mgal} / \mathrm{d}$ for aquaculture, about 57 percent of the total and about 67 percent of the surface-water withdrawals for aquaculture. Louisiana, California, Alaska, and Arkansas each used more than $100 \mathrm{Mgal} / \mathrm{d}$ of groundwater and combined accounted for 57 percent of the total groundwater withdrawals for aquaculture.
Table 9. Aquaculture water withdrawals, 2015.

[Values may not sum to totals because of independent rounding. Values include fresh and saline-water withdrawals]

\begin{tabular}{|c|c|c|c|}
\hline \multirow{3}{*}{ State } & \multicolumn{3}{|c|}{$\begin{array}{c}\text { Withdrawals } \\
\text { (million gallons per day) }\end{array}$} \\
\hline & \multicolumn{2}{|c|}{ By source } & \multirow{2}{*}{ Total } \\
\hline & Groundwater & Surface water & \\
\hline Alabama ............................ & 27.2 & 22.2 & 49.4 \\
\hline 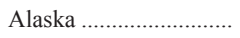 & 169 & 240 & 410 \\
\hline 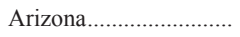 & 23.8 & 10.7 & 34.5 \\
\hline 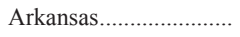 & 152 & 98.9 & 251 \\
\hline California ......................... & 247 & 480 & 727 \\
\hline Colorado......................... & 16.0 & 244 & 260 \\
\hline Connecticut ...................... & 9.15 & 16.0 & 25.2 \\
\hline Delaware ......................... & 1.98 & 0 & 1.98 \\
\hline District of Columbia ... & 0 & 0 & 0 \\
\hline Florida ............................... & 12.8 & 2.10 & 15.0 \\
\hline Georgia.......................... & 14.5 & 70.6 & 85.1 \\
\hline 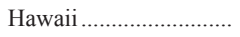 & 8.58 & 10.0 & 18.6 \\
\hline 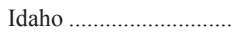 & 46.2 & 1,920 & 1,960 \\
\hline 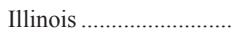 & 5.23 & 5.47 & 10.7 \\
\hline 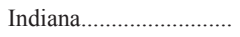 & 6.46 & 8.00 & 14.5 \\
\hline 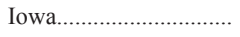 & 7.46 & 12.2 & 19.7 \\
\hline 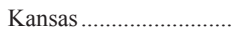 & 3.16 & 3.23 & 6.39 \\
\hline 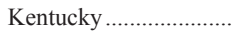 & 0.58 & 47.8 & 48.4 \\
\hline Louisiana.......................... & 337 & 156 & 493 \\
\hline Maine ............................. & 10.9 & 43.1 & 54.0 \\
\hline Maryland ......................... & 5.91 & 2.07 & 7.98 \\
\hline Massachusetts ............... & 8.13 & 1.80 & 9.93 \\
\hline Michigan ........................ & 1.06 & 72.8 & 73.8 \\
\hline Minnesota......................... & 1.72 & 14.8 & 16.5 \\
\hline Mississippi .................... & 87.5 & 39.3 & 127 \\
\hline Missouri …..................... & 6.77 & 157 & 164 \\
\hline Montana ......................... & 3.49 & 13.6 & 17.1 \\
\hline Nebraska ......................... & 10.5 & 16.0 & 26.5 \\
\hline Nevada ............................ & 11.6 & 22.4 & 34.0 \\
\hline New Hampshire ........... & 6.46 & 10.7 & 17.2 \\
\hline New Jersey ....................... & 9.78 & 0 & 9.78 \\
\hline New Mexico.................... & 18.7 & 5.45 & 24.1 \\
\hline New York ......................... & 11.0 & 49.5 & 60.4 \\
\hline North Carolina ............. & 13.3 & 990 & 1,000 \\
\hline North Dakota.................. & 0 & 5.32 & 5.32 \\
\hline Ohio............................... & 13.3 & 26.0 & 39.3 \\
\hline Oklahoma ....................... & 0.06 & 3.23 & 3.29 \\
\hline 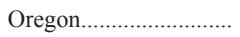 & 32.8 & 601 & 634 \\
\hline Pennsylvania ................... & 48.8 & 47.2 & 96.0 \\
\hline Rhode Island .................. & 6.72 & 0.09 & 6.81 \\
\hline South Carolina .............. & 0.61 & 6.08 & 6.69 \\
\hline South Dakota.................... & 3.57 & 24.9 & 28.5 \\
\hline Tennessee ......................... & 11.7 & 45.2 & 56.9 \\
\hline 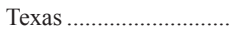 & 11.6 & 11.6 & 23.2 \\
\hline 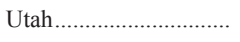 & 83.1 & 0 & 83.1 \\
\hline 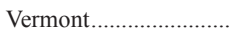 & 4.17 & 7.84 & 12.0 \\
\hline 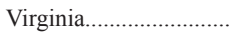 & 0.15 & 113 & 113 \\
\hline Washington...................... & 50.6 & 194 & 245 \\
\hline West Virginia..................... & 8.10 & 31.2 & 39.3 \\
\hline 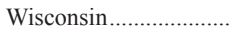 & 25.5 & 22.6 & 48.2 \\
\hline 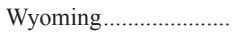 & 4.92 & 23.9 & 28.8 \\
\hline Puerto Rico....................... & 0 & 0.63 & 0.63 \\
\hline U.S. Virgin Islands ...... & 0 & 0 & 0 \\
\hline TOTAL & 1,600 & 5,950 & 7,550 \\
\hline
\end{tabular}



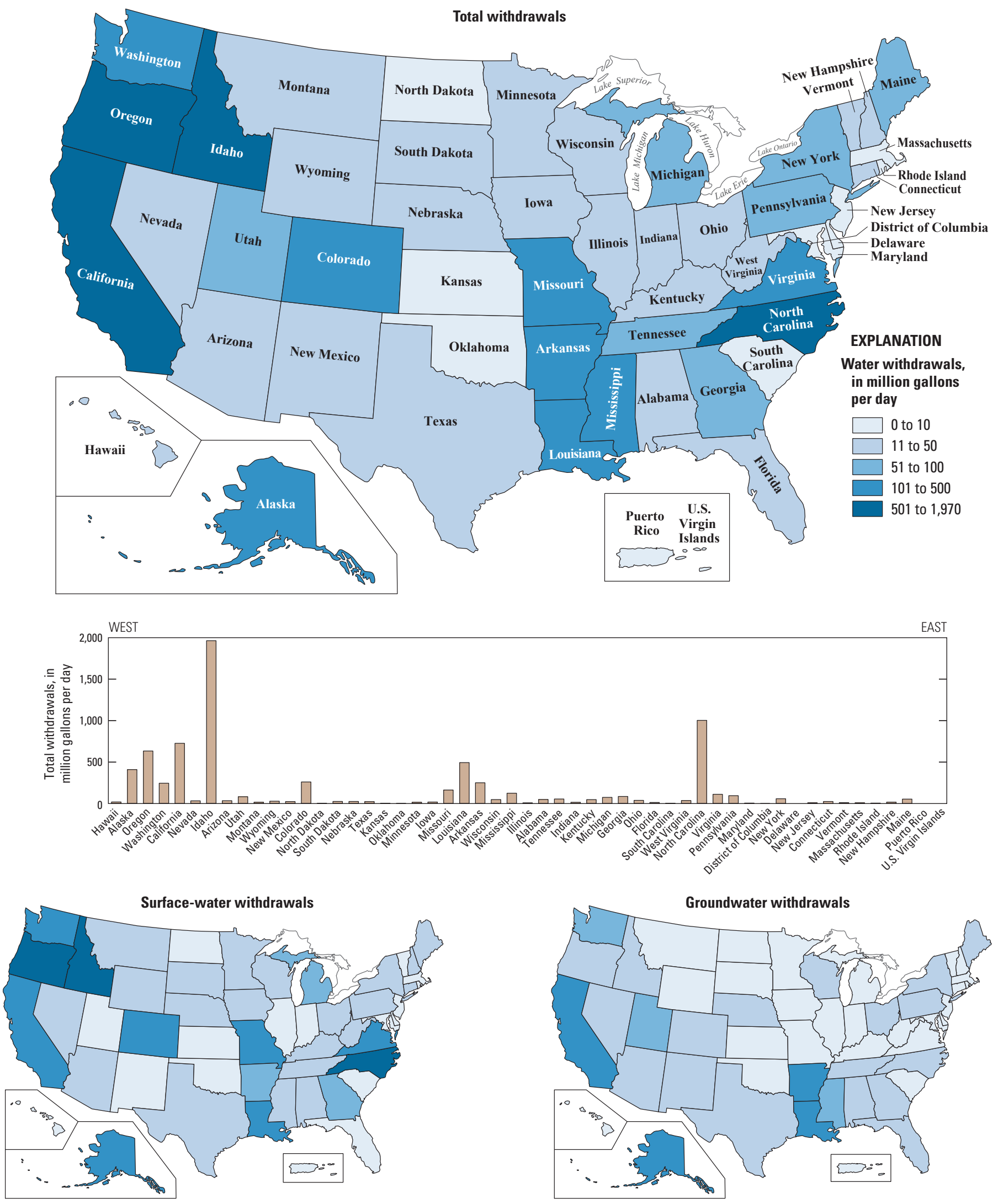

Figure 10. Aquaculture withdrawals by source and State, 2015. 
Industrial withdrawals provide water for such purposes as fabricating, processing, washing, diluting, cooling, or transporting a product; incorporating water into a product; or sanitation needs within the manufacturing facility. Some industries that use large amounts of water produce such commodities as food, paper, chemicals, refined petroleum, or primary metals. Water for industrial use may be delivered from a public supplier or be self-supplied. In this report, industrial use refers to self-supplied industrial withdrawals only. Water withdrawals associated with industrial users that receive water from public suppliers are included in the Public Supply category (table 5, "All other uses and system losses" column). Withdrawals were reported as freshwater or saline water. As in the 2005 and 2010 reports, estimates for public-supply deliveries for industrial and consumptive uses for 2015 were not mandatory for 2015 and are not discussed in this report.

Industrial withdrawals are listed by State in table 10 . For 2015, withdrawals were an estimated $14,800 \mathrm{Mgal} / \mathrm{d}$, or 16,600 thousand acre-ft/yr (table $2 B$ ). Industrial withdrawals were about 5 percent of total withdrawals and about 8 percent of total withdrawals for all categories, excluding thermoelectric power. Surface water was the source for 82 percent of total industrial withdrawals; 94 percent of the surface-water withdrawals for industrial use was freshwater. More than 98 percent of the groundwater withdrawals for industrial use was freshwater. Industrial water withdrawals in the United States decreased 9 percent from 2010 to 2015.

The geographic distribution of total, total surface-water, and total groundwater withdrawals for industrial use is shown in figure 11. Indiana, Louisiana, and Texas accounted for 36 percent of total industrial withdrawals, and Indiana and Louisiana accounted for 36 percent of the total fresh surface-water withdrawals for industrial use. Leading sectors in these States likely contributing to water use in the industrial processes include auto manufacturing (Indiana), medical/ orthopedic devices (Indiana), pharmaceuticals (Indiana) (Groenfeldt, 2015), petroleum refining (Louisiana and Texas), chemicals and petrochemicals (Texas and Louisiana) (Jones, 2015; Downie, 2016) and electronics (Texas) (Downie, 2016). Although it is not a leading sector in Indiana, metals are processed within the State to provide the materials, namely steel and aluminum, to support auto and appliance manufacture (Clark County Indiana Community Portal, 2018). The processing of these metals contributes to industrial water use in Indiana (Indiana Department of Natural Resources, 2016). Texas accounted for 81 percent of the saline surface-water industrial withdrawals, primarily from counties along the Gulf coast. The largest fresh groundwater industrial withdrawals were in California (397 Mgal/d), which accounted for 15 percent of the total national fresh groundwater industrial withdrawals (table 10). The largest groundwater saline withdrawals for industrial purposes in 2015 were in Utah $(36.6 \mathrm{Mgal} / \mathrm{d})$ (table 10), which accounted for 85 percent of saline groundwater withdrawals for industrial use in the United States (table 10). Texas and West Virginia each accounted for about one-half of the remaining 15 percent of saline groundwater withdrawals for industrial use (table 10).

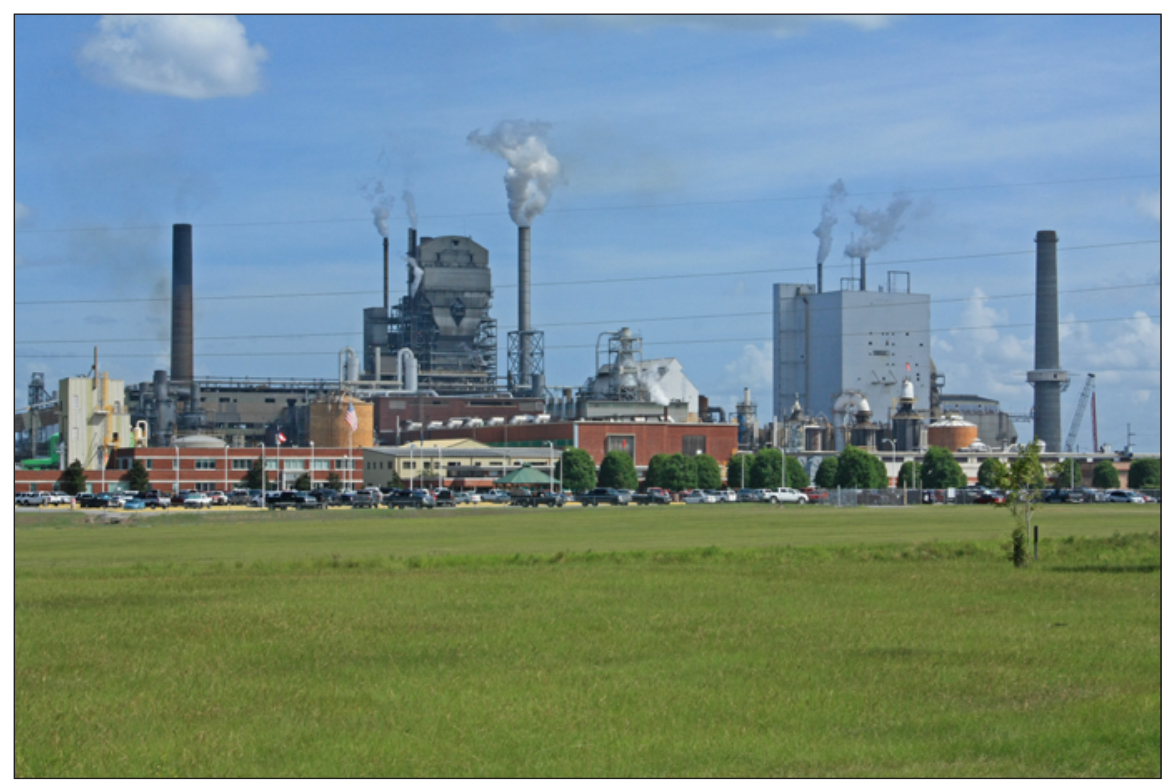

Pulp mill. Photograph by Alan Cressler, USGS. 
Table 10. Industrial self-supplied water withdrawals, 2015.

[Values may not sum to totals because of independent rounding]

\begin{tabular}{|c|c|c|c|c|c|c|c|c|c|}
\hline \multirow{4}{*}{ State } & \multicolumn{9}{|c|}{ Withdrawals (million gallons per day) } \\
\hline & \multicolumn{6}{|c|}{ By source and type } & \multirow{2}{*}{\multicolumn{3}{|c|}{ Total }} \\
\hline & \multicolumn{3}{|c|}{ Groundwater } & \multicolumn{3}{|c|}{ Surface water } & & & \\
\hline & Fresh & Saline & Total & Fresh & Saline & Total & Fresh & Saline & Total \\
\hline Alabama ........................... & 32.7 & 0 & 32.7 & 461 & 0 & 461 & 494 & 0 & 494 \\
\hline Alaska ............................ & 6.70 & 0 & 6.70 & 1.65 & 1.83 & 3.48 & 8.35 & 1.83 & 10.2 \\
\hline Arizona............................. & 6.12 & 0 & 6.12 & 0 & 0 & 0 & 6.12 & 0 & 6.12 \\
\hline Arkansas........................... & 29.5 & 0 & 29.5 & 127 & 0 & 127 & 157 & 0 & 157 \\
\hline California ....................... & 397 & 0 & 397 & 1.13 & 0 & 1.13 & 399 & 0 & 399 \\
\hline Colorado........................... & 4.22 & 0 & 4.22 & 79.9 & 0 & 79.9 & 84.1 & 0 & 84.1 \\
\hline Connecticut ..................... & 40.1 & 0 & 40.1 & 141 & 41.6 & 182 & 181 & 41.6 & 222 \\
\hline Delaware .......................... & 10.7 & 0 & 10.7 & 291 & 0 & 291 & 302 & 0 & 302 \\
\hline District of Columbia ... & 0 & 0 & 0 & 0 & 0 & 0 & 0 & 0 & 0 \\
\hline 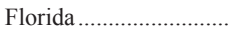 & 181 & 0 & 181 & 63.6 & 1.08 & 64.7 & 245 & 1.08 & 246 \\
\hline 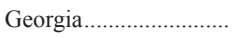 & 193 & 0 & 193 & 283 & 0 & 283 & 475 & 0 & 475 \\
\hline 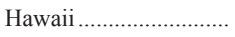 & 0.24 & 0 & 0.24 & 0 & 0 & 0 & 0.24 & 0 & 0.24 \\
\hline 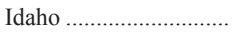 & 45.0 & 0 & 45.0 & 12.7 & 0 & 12.7 & 57.6 & 0 & 57.6 \\
\hline 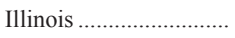 & 132 & 0 & 132 & 299 & 0 & 299 & 431 & 0 & 431 \\
\hline 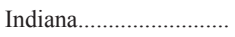 & 79.9 & 0 & 79.9 & 2,210 & 0 & 2,210 & 2,290 & 0 & 2,290 \\
\hline 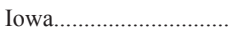 & 92.8 & 0 & 92.8 & 195 & 0 & 195 & 288 & 0 & 288 \\
\hline 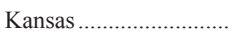 & 30.5 & 0 & 30.5 & 7.63 & 0 & 7.63 & 38.1 & 0 & 38.1 \\
\hline 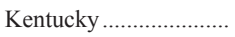 & 77.3 & 0 & 77.3 & 148 & 0 & 148 & 225 & 0 & 225 \\
\hline Louisiana.......................... & 250 & 0 & 250 & 1,890 & 0 & 1,890 & 2,140 & 0 & 2,140 \\
\hline 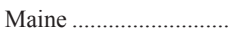 & 5.83 & 0 & 5.83 & 176 & 42.9 & 219 & 182 & 42.9 & 225 \\
\hline Maryland .......................... & 10.8 & 0 & 10.8 & 38.6 & 1.37 & 40.0 & 49.4 & 1.37 & 50.8 \\
\hline Massachusetts ............... & 15.2 & 0 & 15.2 & 12.7 & 0 & 12.7 & 27.9 & 0 & 27.9 \\
\hline Michigan ......................... & 64.6 & 0 & 64.6 & 453 & 0 & 453 & 518 & 0 & 518 \\
\hline Minnesota......................... & 49.0 & 0 & 49.0 & 210 & 0 & 210 & 259 & 0 & 259 \\
\hline Mississippi ...................... & 72.8 & 0 & 72.8 & 109 & 0 & 109 & 182 & 0 & 182 \\
\hline 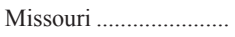 & 42.4 & 0 & 42.4 & 42.8 & 0 & 42.8 & 85.2 & 0 & 85.2 \\
\hline 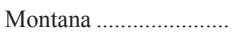 & 5.22 & 0 & 5.22 & 4.45 & 0 & 4.45 & 9.67 & 0 & 9.67 \\
\hline Nebraska ......................... & 42.0 & 0 & 42.0 & 2.32 & 0 & 2.32 & 44.3 & 0 & 44.3 \\
\hline 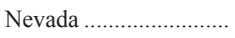 & 1.02 & 0 & 1.02 & 4.69 & 0 & 4.69 & 5.71 & 0 & 5.71 \\
\hline New Hampshire ........... & 4.21 & 0 & 4.21 & 8.41 & 0 & 8.41 & 12.6 & 0 & 12.6 \\
\hline New Jersey ..................... & 30.0 & 0 & 30.0 & 64.1 & 0 & 64.1 & 94.1 & 0 & 94.1 \\
\hline New Mexico..................... & 3.40 & 0 & 3.40 & 0 & 0 & 0 & 3.40 & 0 & 3.40 \\
\hline New York ......................... & 29.4 & 0 & 29.4 & 283 & 8.43 & 291 & 312 & 8.43 & 320 \\
\hline North Carolina .............. & 15.0 & 0 & 15.0 & 178 & 0 & 178 & 193 & 0 & 193 \\
\hline North Dakota.................... & 6.18 & 0 & 6.18 & 13.5 & 0 & 13.5 & 19.6 & 0 & 19.6 \\
\hline 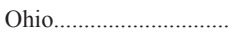 & 138 & 0 & 138 & 210 & 0 & 210 & 348 & 0 & 348 \\
\hline 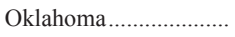 & 7.27 & 0 & 7.27 & 44.7 & 0 & 44.7 & 52.0 & 0 & 52.0 \\
\hline 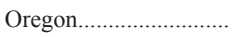 & 3.45 & 0 & 3.45 & 101 & 0 & 101 & 105 & 0 & 105 \\
\hline Pennsylvania ................... & 45.9 & 0 & 45.9 & 599 & 0 & 599 & 645 & 0 & 645 \\
\hline Rhode Island ................... & 0.54 & 0 & 0.54 & 1.51 & 0.03 & 1.54 & 2.05 & 0.03 & 2.08 \\
\hline South Carolina .............. & 26.3 & 0 & 26.3 & 260 & 0 & 260 & 286 & 0 & 286 \\
\hline South Dakota..................... & 18.4 & 0 & 18.4 & 6.04 & 0 & 6.04 & 24.4 & 0 & 24.4 \\
\hline Tennessee ........................ & 51.6 & 0 & 51.6 & 682 & 0 & 682 & 734 & 0 & 734 \\
\hline 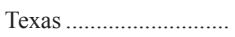 & 99.8 & 3.28 & 103 & 223 & 598 & 821 & 323 & 601 & 924 \\
\hline 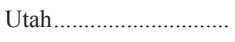 & 37.4 & 36.6 & 74.0 & 16.8 & 42.4 & 59.2 & 54.2 & 79.0 & 133 \\
\hline 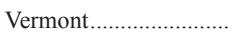 & 1.51 & 0 & 1.51 & 9.46 & 0 & 9.46 & 11.0 & 0 & 11.0 \\
\hline Virginia............................ & 66.0 & 0 & 66.0 & 304 & 5.15 & 309 & 370 & 5.15 & 375 \\
\hline 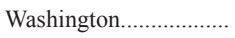 & 83.5 & 0 & 83.5 & 329 & 0 & 329 & 412 & 0 & 412 \\
\hline West Virginia..................... & 25.7 & 3.05 & 28.7 & 399 & 0 & 399 & 424 & 3.05 & 427 \\
\hline Wisconsin........................ & 46.8 & 0 & 46.8 & 336 & 0 & 336 & 382 & 0 & 382 \\
\hline 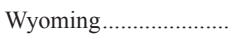 & 5.83 & 0 & 5.83 & 2.21 & 0 & 2.21 & 8.04 & 0 & 8.04 \\
\hline Puerto Rico....................... & 3.67 & 0 & 3.67 & 0 & 0 & 0 & 3.67 & 0 & 3.67 \\
\hline U.S. Virgin Islands ....... & 0.52 & 0 & 0.52 & 0 & 0 & 0 & 0.52 & 0 & 0.52 \\
\hline TOTAL & 2,670 & 42.9 & 2,710 & 11,300 & 743 & 12,100 & 14,000 & 786 & 14,800 \\
\hline
\end{tabular}



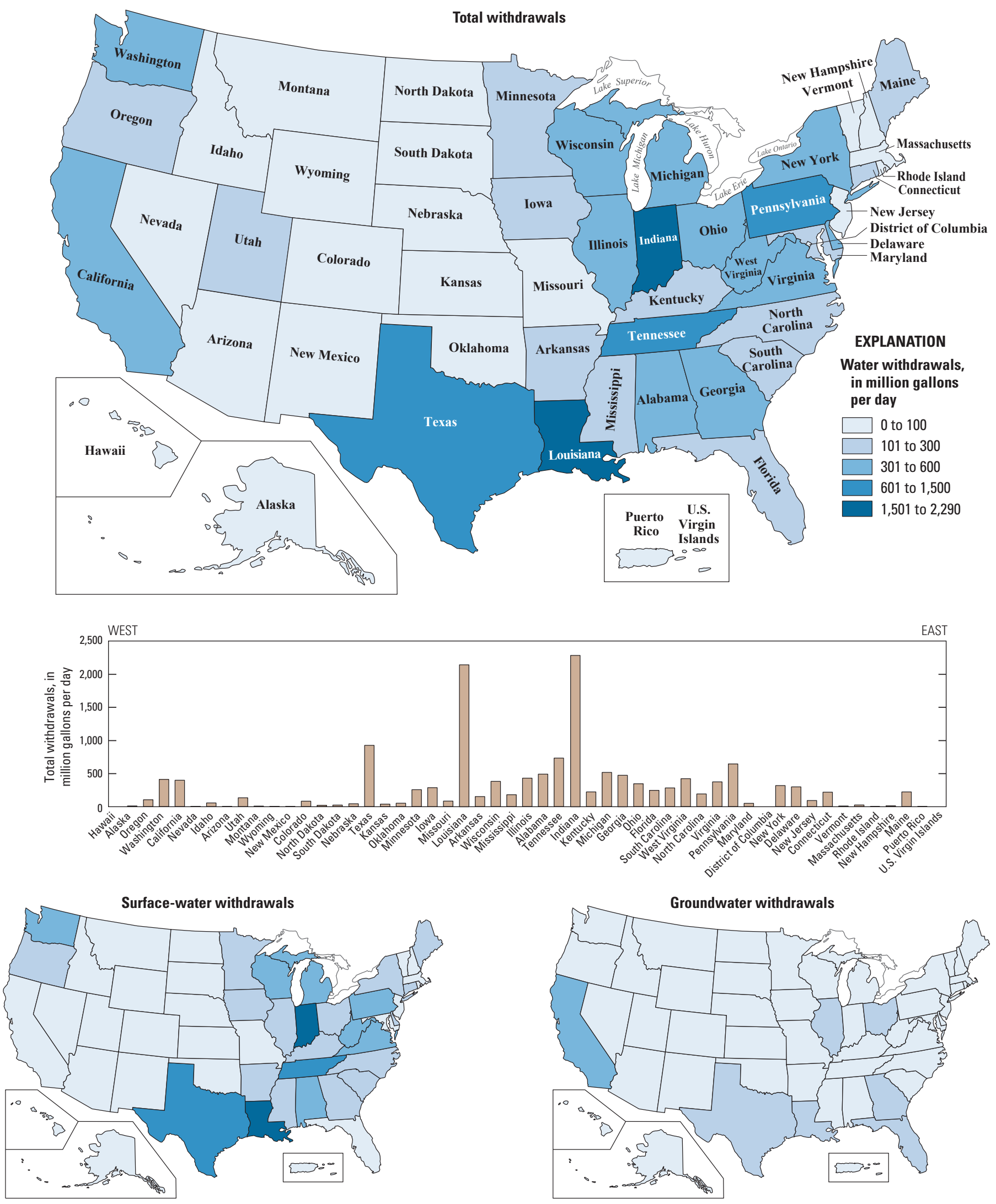

Figure 11. Industrial withdrawals by source and State, 2015. 
Mining water use is water used for the extraction of minerals and rocks that may be in the form of solids, such as coal, iron, sand, and gravel; liquids, such as crude petroleum; and gases, such as natural gas. The category includes quarrying, milling of mined materials, injection of water for secondary oil recovery or for unconventional oil and gas recovery (such as hydraulic fracturing), and other operations associated with mining activities. All mining withdrawals were considered to be self-supplied. Water withdrawals were reported as freshwater or saline water. Dewatering was not reported as a mining withdrawal unless the water was used beneficially, such as dampening roads for dust control.

Sources of data used to estimate water use for mining include surveys of mining operations and State and Federal agencies that collect water withdrawal, discharge, or mineral production data for mining operations. Many of the 2015 withdrawals for mining were estimated according to methods described by Lovelace (2009c), using mineral production data and water-use coefficients, in gallons per weight or volume of minerals produced. Production data for nonfuel minerals, including metals and nonmetallic minerals, were provided by the USGS National Minerals Information Center. Production or water-injection data for fuel minerals, including coal, petroleum, and natural gas, were obtained from the USDOE EIA, the FracFocus Chemical

Disclosure Registry

(FracFocus, 2016), and various State agencies.

Mining withdrawals during 2015 are listed by State in table 11. During 2015, an estimated 4,000 Mgal/d, or 4,480 thousand acre-ft/yr (table 2B), were withdrawn. Mining withdrawals were about 1 percent of total withdrawals and about 2 percent of total withdrawals for all categories, excluding thermoelectric power. Groundwater was the source for 72 percent of total withdrawals for mining (table 11).

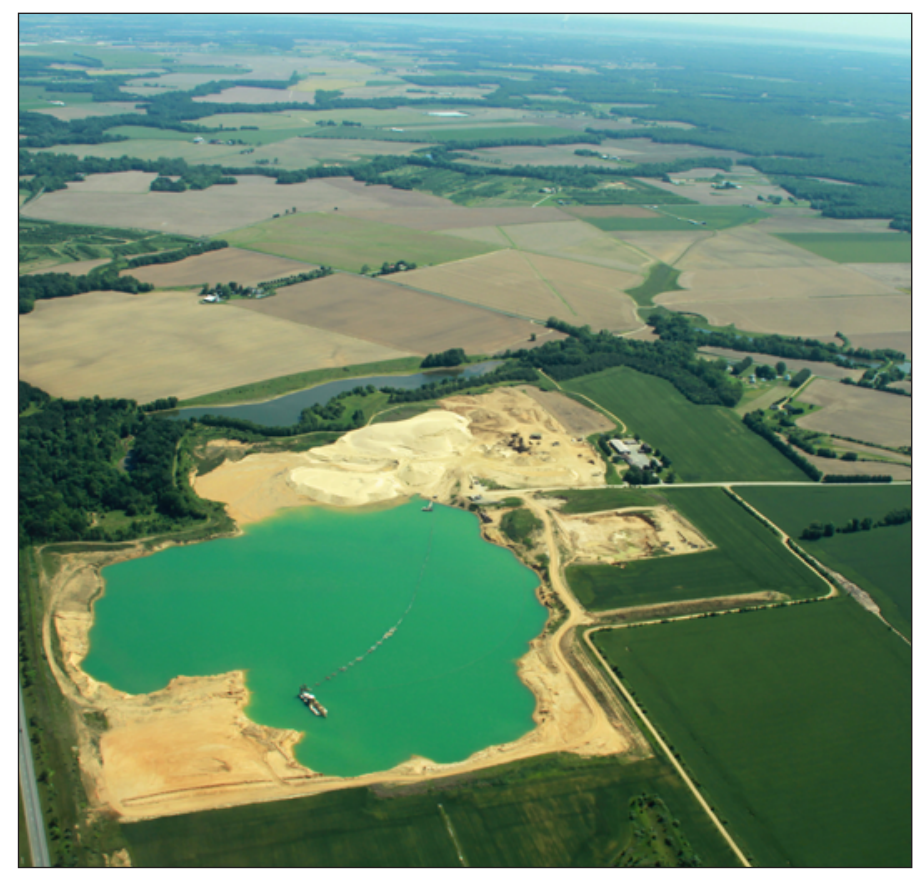

Sixty-five percent of the groundwater withdrawn for mining was saline. Seventy-seven percent of the surface-water withdrawn was freshwater. Saline groundwater withdrawals and fresh surface-water withdrawals together represented 68 percent of the total withdrawals for mining (table 11). Total mining withdrawals in 2015 were about 1 percent more than in 2010. Groundwater withdrawals were 1 percent more, and surface-water withdrawals were less than 1 percent less. Freshwater withdrawals in 2015 were 4 percent less than in 2010 , and saline-water withdrawals were 5 percent more than in 2010 .

The geographic distribution of total, total freshwater, and total saline-water withdrawals is shown in figure 12 . Total mining withdrawals in Texas, including $1,000 \mathrm{Mgal} / \mathrm{d}$ of saline groundwater, were 1,140 Mgal/d and accounted for 28 percent of the total withdrawals for mining (table 11). The next largest users, California, Utah, and Nevada each used greater than $200 \mathrm{Mgal} / \mathrm{d}$ of water for mining and accounted for another 20 percent of total withdrawals (fig. 12; table 11). Nevada, Texas, Ohio, Florida, and Arizona each used more than $50 \mathrm{Mgal} / \mathrm{d}$ of fresh groundwater for mining and together accounted for 55 percent of total fresh groundwater withdrawals for this use. Indiana, Iowa, Michigan, and New Jersey each used more than $50 \mathrm{Mgal} / \mathrm{d}$ of fresh surface water for mining and together accounted for 37 percent of total fresh surface-water withdrawals for this use (table 11). Saline groundwater withdrawals were greater than $150 \mathrm{Mgal} / \mathrm{d}$ in Texas, California, and Oklahoma and together accounted for 77 percent of total saline groundwater withdrawals for mining. Saline surfacewater withdrawals were greater than $40 \mathrm{Mgal} / \mathrm{d}$ for Utah and Alaska and together accounted for almost 100 percent of total saline surface-water withdrawals for mining (table 11).

Wash pond. Photograph by Wendy McPherson, USGS 
Table 11. Mining water withdrawals, 2015.

[Values may not sum to totals because of independent rounding]

\begin{tabular}{|c|c|c|c|c|c|c|c|c|c|}
\hline \multirow{4}{*}{ State } & \multicolumn{9}{|c|}{ Withdrawals (million gallons per day) } \\
\hline & \multicolumn{6}{|c|}{ By source and type } & \multirow{2}{*}{\multicolumn{3}{|c|}{ Total }} \\
\hline & \multicolumn{3}{|c|}{ Groundwater } & \multicolumn{3}{|c|}{ Surface water } & & & \\
\hline & Fresh & Saline & Total & Fresh & Saline & Total & Fresh & Saline & Total \\
\hline Alabama ................................ & 21.8 & 0 & 21.8 & 8.45 & 0 & 8.45 & 30.2 & 0 & 30.2 \\
\hline Alaska ............................. & 0 & 89.2 & 89.2 & 36.4 & 41.4 & 77.8 & 36.4 & 131 & 167 \\
\hline Arizona............................. & 68.3 & 0 & 68.3 & 0 & 0 & 0 & 68.3 & 0 & 68.3 \\
\hline Arkansas............................ & 0.14 & 0 & 0.14 & 2.93 & 0 & 2.93 & 3.07 & 0 & 3.07 \\
\hline California ....................... & 30.2 & 272 & 302 & 15.6 & 0.01 & 15.6 & 45.8 & 272 & 318 \\
\hline Colorado............................ & 5.24 & 24.2 & 29.4 & 2.46 & 0 & 2.46 & 7.70 & 24.2 & 31.9 \\
\hline 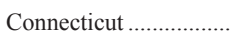 & 0.82 & 0 & 0.82 & 3.43 & 0 & 3.43 & 4.25 & 0 & 4.25 \\
\hline 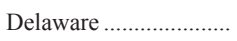 & 0.34 & 0 & 0.34 & 0.31 & 0 & 0.31 & 0.65 & 0 & 0.65 \\
\hline District of Columbia ... & 0 & 0 & 0 & 0 & 0 & 0 & 0 & 0 & 0 \\
\hline Florida & 89.1 & 0 & 89.1 & 40.7 & 0 & 40.7 & 130 & 0 & 130 \\
\hline 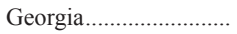 & 15.9 & 0 & 15.9 & 3.89 & 0 & 3.89 & 19.8 & 0 & 19.8 \\
\hline Hawaii ................................. & 0.89 & 0 & 0.89 & 0.03 & 0 & 0.03 & 0.92 & 0 & 0.92 \\
\hline Idaho & 1.35 & 0 & 1.35 & 21.8 & 0 & 21.8 & 23.1 & 0 & 23.1 \\
\hline Illinois & 14.6 & 21.0 & 35.6 & 40.4 & 0 & 40.4 & 55.0 & 21.0 & 76.0 \\
\hline Indiana................................. & 5.52 & 0 & 5.52 & 121 & 0 & 121 & 126 & 0 & 126 \\
\hline 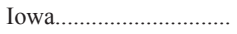 & 1.08 & 0 & 1.08 & 74.4 & 0 & 74.4 & 75.5 & 0 & 75.5 \\
\hline 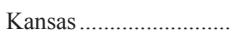 & 5.44 & 0 & 5.44 & 0.55 & 0 & 0.55 & 5.99 & 0 & 5.99 \\
\hline 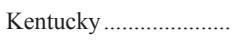 & 17.6 & 0 & 17.6 & 22.9 & 0 & 22.9 & 40.6 & 0 & 40.6 \\
\hline Louisiana ............................. & 1.65 & 0 & 1.65 & 4.59 & 0 & 4.59 & 6.24 & 0 & 6.24 \\
\hline 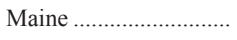 & 1.45 & 0 & 1.45 & 4.88 & 0 & 4.88 & 6.33 & 0 & 6.33 \\
\hline Maryland ............................. & 13.7 & 0 & 13.7 & 3.09 & 0 & 3.09 & 16.8 & 0 & 16.8 \\
\hline Massachusetts ............... & 5.51 & 0 & 5.51 & 4.09 & 0 & 4.09 & 9.60 & 0 & 9.60 \\
\hline Michigan ............................ & 16.0 & 0.58 & 16.6 & 69.7 & 0 & 69.7 & 85.7 & 0.58 & 86.2 \\
\hline Minnesota........................... & 3.63 & 0 & 3.63 & 5.57 & 0 & 5.57 & 9.20 & 0 & 9.20 \\
\hline Mississippi ........................ & 8.21 & 6.58 & 14.8 & 1.24 & 0 & 1.24 & 9.45 & 6.58 & 16.0 \\
\hline Missouri …......................... & 21.1 & 0 & 21.1 & 8.47 & 0 & 8.47 & 29.6 & 0 & 29.6 \\
\hline Montana .............................. & 1.06 & 16.3 & 17.4 & 20.5 & 0 & 20.5 & 21.6 & 16.3 & 37.9 \\
\hline Nebraska ........................... & 0.06 & 6.41 & 6.47 & 9.54 & 0 & 9.54 & 9.60 & 6.41 & 16.0 \\
\hline 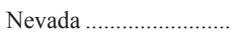 & 187 & 11.3 & 199 & 8.13 & 0 & 8.13 & 195 & 11.3 & 207 \\
\hline New Hampshire ............ & 1.76 & 0 & 1.76 & 4.37 & 0 & 4.37 & 6.13 & 0 & 6.13 \\
\hline New Jersey ........................ & 1.48 & 0 & 1.48 & 56.8 & 0 & 56.8 & 58.3 & 0 & 58.3 \\
\hline New Mexico ....................... & 39.4 & 89.4 & 129 & 17.4 & 0 & 17.4 & 56.8 & 89.4 & 146 \\
\hline New York ........................... & 5.64 & 0.95 & 6.59 & 34.6 & 0 & 34.6 & 40.2 & 0.95 & 41.2 \\
\hline North Carolina ............... & 30.0 & 0 & 30.0 & 8.26 & 0 & 8.26 & 38.3 & 0 & 38.3 \\
\hline North Dakota.................... & 26.2 & 15.1 & 41.3 & 4.50 & 0 & 4.50 & 30.7 & 15.1 & 45.8 \\
\hline Ohio..................................... & 91.3 & 0 & 91.3 & 37.6 & 0 & 37.6 & 129 & 0 & 129 \\
\hline 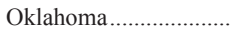 & 4.22 & 155 & 159 & 33.2 & 0 & 33.2 & 37.4 & 155 & 192 \\
\hline Oregon............................... & 8.45 & 0 & 8.45 & 2.85 & 0 & 2.85 & 11.3 & 0 & 11.3 \\
\hline Pennsylvania ..................... & 32.9 & 5.60 & 38.5 & 5.21 & 0 & 5.21 & 38.1 & 5.60 & 43.7 \\
\hline Rhode Island .................... & 1.18 & 0 & 1.18 & 1.74 & 0 & 1.74 & 2.92 & 0 & 2.92 \\
\hline South Carolina ................... & 8.28 & 0 & 8.28 & 1.79 & 0 & 1.79 & 10.1 & 0 & 10.1 \\
\hline South Dakota..................... & 3.59 & 0 & 3.59 & 5.06 & 0 & 5.06 & 8.65 & 0 & 8.65 \\
\hline Tennessee .......................... & 17.1 & 0 & 17.1 & 14.2 & 0 & 14.2 & 31.4 & 0 & 31.4 \\
\hline Texas ................................. & 116 & 1,000 & 1,120 & 15.9 & 0.01 & 15.9 & 131 & 1,000 & 1,140 \\
\hline Utah & 1.67 & 43.7 & 45.4 & 1.80 & 214 & 216 & 3.47 & 258 & 262 \\
\hline Vermont.............................. & 0.23 & 0 & 0.23 & 4.33 & 0 & 4.33 & 4.56 & 0 & 4.56 \\
\hline Virginia................................. & 6.20 & 0 & 6.20 & 18.7 & 0 & 18.7 & 24.9 & 0 & 24.9 \\
\hline 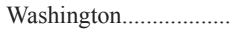 & 13.4 & 0 & 13.4 & 3.61 & 0 & 3.61 & 17.0 & 0 & 17.0 \\
\hline West Virginia...................... & 23.8 & 1.52 & 25.3 & 29.5 & 0 & 29.5 & 53.3 & 1.52 & 54.9 \\
\hline Wisconsin & 0.42 & 0 & 0.42 & 28.8 & 0 & 28.8 & 29.3 & 0 & 29.3 \\
\hline 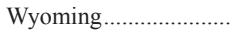 & 33.1 & 96.8 & 130 & 11.4 & 0 & 11.4 & 44.5 & 96.8 & 141 \\
\hline Puerto Rico....................... & 1.84 & 0 & 1.84 & 0.18 & 0 & 0.18 & 2.02 & 0 & 2.02 \\
\hline$\underline{\text { U.S. Virgin Islands ....... }}$ & 0 & 0 & 0 & 0 & 0.02 & 0.02 & 0 & 0.02 & 0.02 \\
\hline TOTAL & 1,010 & 1,860 & 2,870 & 877 & 256 & 1,130 & 1,880 & 2,120 & 4,000 \\
\hline
\end{tabular}



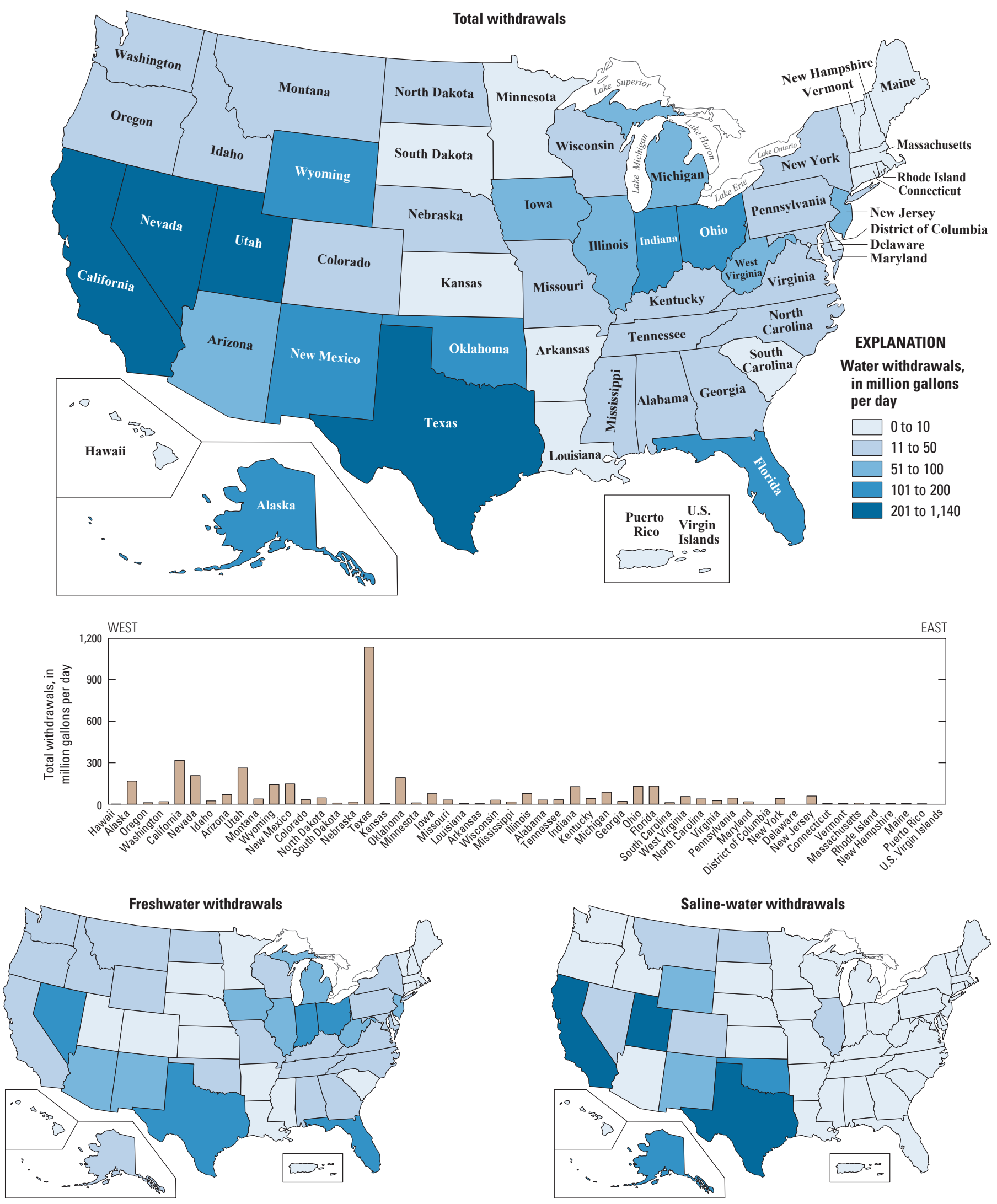

Figure 12. Mining withdrawals by water quality and State, 2015. 
Water withdrawn for thermoelectric-power generation is primarily used to condense, or cool, the steam used to drive thermoelectric generators. In general, there are two types of cooling systems used for thermoelectric power: once-through and recirculating. Once-through (also known as open-loop) cooling systems withdraw relatively large volumes of water, route the water through condensers to cool the steam used to generate electricity, and then return the water at a higher temperature. The heated discharge results in evaporative losses of the water, which for a once-through cooling system is the consumptive use of water. Consumptive use is relatively small compared to the total amount of water withdrawn for a once-through cooling system. Recirculating (also known as closed-loop or recirculation) cooling systems withdraw relatively smaller volumes of water (compared to oncethrough cooling systems) and circulate the water between the condensers and the cooling system, either recirculating towers or cooling ponds. Subsequent water withdrawals are used to replace, or make up, water lost to evaporation, blowdown, drift, and leakage. Consumptive use for a recirculating cooling system is the water lost to evaporation in cooling towers or from the surface of recirculating ponds and is relatively large (compared to the total amount of water withdrawn).

Sources of information used to compile the thermoelectricpower category data include State water agencies, powerplant facilities, USDOE EIA, USGS thermoelectric water-use project, and coefficients derived from previous USGS compilations. The USGS thermoelectric water-use project estimated monthly and annual water withdrawals, and consumptive use for 2015, on the basis of linked heat-and-water budget models that are constrained by power-generation technologies, cooling-system technologies, and environmental variables, such as air and water temperatures, wind speeds, and elevation (Diehl and others, 2013; Diehl and Harris, 2014). The USGS estimates include minimum to maximum ranges of withdrawals and consumptive use, as well as plant-level "best" estimates. These plausible, thermodynamically based model estimates provided a quality-assurance check for reported data. The monthly and annual model estimates were provided to NWUSP compilers as supplemental and supportive datasets for the compilation and included cooling-system-type categories and water sources. USDOE EIA-reported water-use data and net power generation data also were provided (U.S. Department of Energy, 2016a, b). These data were used either in whole or in part for this compilation. Compilers in some States obtained data reported directly from thermoelectric powerplants.

Thermoelectric-power withdrawals and consumptive-use data were compiled according to once-through and recirculating cooling-system types, and reported as freshwater or saline water from surface water or groundwater sources. Net power generation was compiled by cooling-system type. Consumptive use for thermoelectric water use was reported for the first time since 1995. Public-supply deliveries to thermoelectric powerplants and the use of reclaimed wastewater for cooling purposes were not required to be reported, although some States did report these data.

Thermoelectric-power withdrawals, consumptive use, and net power generation are listed by State in table 12 . Total withdrawals for thermoelectric power for 2015 were $133,000 \mathrm{Mgal} / \mathrm{d}$, or 149,000 thousand acre-ft/yr (table 2B). Surface water was the source for nearly 100 percent of total thermoelectric-power withdrawals, and 72 percent of those surface-water withdrawals were from freshwater sources. Saline surface-water withdrawals for thermoelectric power accounted for 97 percent of total saline surface-water withdrawals for all uses. Total withdrawals for thermoelectric power accounted for 41 percent of total water withdrawals, 34 percent of total freshwater withdrawals, and 48 percent of fresh surface-water withdrawals for all uses. Total thermoelectric-power consumptive use for 2015 was 4,310 Mgal/d, about 3 percent of total thermoelectric-power withdrawals. Freshwater accounted for 87 percent of total consumptive use. Net power generation associated with thermoelectric-power water use was 3,230,000 gigawatt-hours ( $\mathrm{gWh}$ ), or 83 percent of the total reported utility power (public utilities and independent power producers) in the United States for 2015 (U.S. Department of Energy, 2016b). On average, 15 gallons (gal) of water was used to produce 1 kilowatt-hour (kWh) of electricity in 2015, compared to almost 19 gallons per kilowatt-hour in 2010.

The geographic distribution of total, total freshwater, and total saline-water withdrawals for thermoelectric power is shown in figure 13. The largest total withdrawals for thermoelectric power were in Texas, where 93 percent of the withdrawals were from freshwater sources. Texas, Illinois, Michigan, Alabama, and North Carolina each withdrew more than $6,000 \mathrm{Mgal} / \mathrm{d}$ of freshwater for electricity generation, which combined accounted for more than 40 percent of freshwater withdrawals for thermoelectric power. Florida, New York, and Maryland accounted for about 53 percent of total saline withdrawals for thermoelectric power, nearly all from surface water. Nevada, California, Florida, and Hawaii accounted for 90 percent of the total saline groundwater withdrawals.

Estimated 2015 thermoelectric withdrawals were 18 percent less than estimates for 2010. Reasons for this large difference include plant closures (U.S. Department of Energy, 2016a), decrease of coal use and increase of natural gas use (U.S. Department of Energy, 2016c), and new powerplants using more water-efficient power generation and coolingsystem technologies (U.S. Department of Energy, 2016a, b).

The conterminous Eastern States (fig. 13) accounted for 84 percent of total thermoelectric-power withdrawals and 70 percent of the related net power generation in the 
Table 12. Thermoelectric-power water use, 2015.

[Values may not sum to totals because of independent rounding. All withdrawal, reclaimed wastewater, delivery, and use data are in million gallons per day. Abbreviation: - , not estimated]

\begin{tabular}{|c|c|c|c|c|c|c|c|c|c|c|c|c|c|c|}
\hline \multirow{4}{*}{ State } & \multicolumn{7}{|c|}{ Withdrawals } & \multirow{4}{*}{$\begin{array}{c}\text { Re- } \\
\text { claimed } \\
\text { waste- } \\
\text { water }\end{array}$} & \multirow{4}{*}{$\begin{array}{c}\text { Public- } \\
\text { supply } \\
\text { deliveries } \\
\text { to thermo- } \\
\text { electric- } \\
\text { power } \\
\text { generation } \\
\text { use }\end{array}$} & \multicolumn{4}{|c|}{ Total use } & \multirow{4}{*}{$\begin{array}{c}\text { Net power } \\
\text { generated } \\
\text { (gigawatt- } \\
\text { hours) }\end{array}$} \\
\hline & \multirow{2}{*}{\multicolumn{2}{|c|}{ Groundwater }} & \multirow{2}{*}{\multicolumn{2}{|c|}{ Surface water }} & \multirow{2}{*}{\multicolumn{3}{|c|}{ Total }} & & & \multirow{3}{*}{$\begin{array}{c}\text { Water use } \\
\text { (with- } \\
\text { drawals, } \\
\text { deliver- } \\
\text { ies, and } \\
\text { reclaimed } \\
\text { waste- } \\
\text { water) } \\
\text { Total }\end{array}$} & \multirow{2}{*}{\multicolumn{3}{|c|}{ Consumptive use }} & \\
\hline & & & & & & & & & & & & & & \\
\hline & Fresh & Saline & Fresh & Saline & Fresh & Saline & Total & & & & Fresh & Saline & Total & \\
\hline Alabama ............................. & 0 & 0 & 6,630 & 0 & 6,630 & 0 & 6,630 & - & - & 6,630 & 107 & 0 & 107 & 128,000 \\
\hline 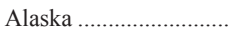 & 0.63 & 0 & 66.1 & 0 & 66.7 & 0 & 66.7 & - & 0.60 & 67.3 & 1.38 & 0 & 1.38 & 1,870 \\
\hline 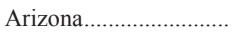 & 57.7 & 0 & 25.8 & 0 & 83.5 & 0 & 83.5 & 67.7 & 0.50 & 152 & 147 & 0 & 147 & 102,000 \\
\hline Arkansas.......................... & 3.13 & 0 & 1,440 & 0 & 1,440 & 0 & 1,440 & - & - & 1,440 & 61.3 & 0 & 61.3 & 49,500 \\
\hline California ........................ & 19.6 & 35.1 & 16.7 & 2,800 & 36.4 & 2,840 & 2,880 & 15.6 & 38.9 & 2,930 & 64.4 & 25.3 & 89.7 & 111,000 \\
\hline Colorado.......................... & 3.03 & 0 & 34.1 & 0 & 37.2 & 0 & 37.2 & 4.11 & 13.7 & 55.0 & 46.7 & 0 & 46.7 & 42,300 \\
\hline Connecticut ...................... & 0 & 0 & 126 & 2,470 & 126 & 2,470 & 2,590 & - & 4.65 & 2,600 & 7.05 & 23.2 & 30.2 & 31,000 \\
\hline Delaware ........................ & 0.13 & 0 & 14.3 & 256 & 14.4 & 256 & 271 & - & - & 271 & 10.3 & 4.02 & 14.3 & 5,660 \\
\hline District of Columbia ... & 0 & 0 & 0 & 0 & 0 & 0 & 0 & 0 & 0 & 0 & 0 & 0 & 0 & 0 \\
\hline Florida ............................. & 27.8 & 28.5 & 406 & 9,400 & 434 & 9,420 & 9,860 & 34.5 & 8.88 & 9,900 & 71.6 & 122 & 194 & 231,000 \\
\hline Georgia.......................... & 3.43 & 0 & 738 & 102 & 741 & 102 & 843 & - & - & 843 & 161 & 0.02 & 161 & 110,000 \\
\hline 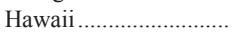 & 1.48 & 20.2 & 0 & 357 & 1.48 & 377 & 379 & - & - & 379 & 0.51 & 3.10 & 3.61 & 7,820 \\
\hline Idaho ................................. & 1.79 & 0 & 0 & 0 & 1.79 & 0 & 1.79 & - & - & 1.79 & 1.79 & 0 & 1.79 & 2,210 \\
\hline 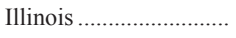 & 4.21 & 0 & 8,130 & 0 & 8,140 & 0 & 8,140 & - & - & 8,140 & 572 & 0 & 572 & 177,000 \\
\hline 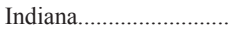 & 20.8 & 0 & 3,800 & 0 & 3,820 & 0 & 3,820 & - & - & 3,820 & 161 & 0 & 161 & 87,000 \\
\hline Iowa................................ & 27.0 & 0 & 1,660 & 0 & 1,680 & 0 & 1,680 & 0.42 & 0.16 & 1,680 & 40.0 & 0 & 40.0 & 35,500 \\
\hline 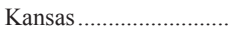 & 7.81 & 0 & 809 & 0 & 817 & 0 & 817 & - & 0.25 & 817 & 54.1 & 0 & 54.1 & 33,800 \\
\hline 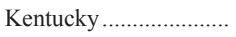 & 4.96 & 0 & 1,860 & 0 & 1,860 & 0 & 1,860 & - & - & 1,860 & 77.1 & 0 & 77.1 & 74,700 \\
\hline Louisiana......................... & 37.0 & 0 & 4,000 & 261 & 4,040 & 261 & 4,300 & - & - & 4,300 & 82.3 & 2.61 & 84.9 & 69,700 \\
\hline 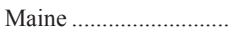 & 0.92 & 0 & 4.38 & 80.7 & 5.30 & 80.7 & 86.0 & - & 2.05 & 88.0 & 4.94 & 1.00 & 5.94 & 4,960 \\
\hline Maryland ......................... & 2.30 & 0 & 218 & 5,300 & 220 & 5,300 & 5,520 & - & - & 5,520 & 2.29 & 61.2 & 63.4 & 30,700 \\
\hline Massachusetts ................ & 1.10 & 0 & 49.0 & 487 & 50.1 & 487 & 537 & 1.07 & 47.0 & 585 & 4.86 & 14.2 & 19.1 & 16,800 \\
\hline Michigan .......................... & 5.61 & 0 & 7,800 & 0 & 7,800 & 0 & 7,800 & - & - & 7,800 & 117 & 0 & 117 & 102,000 \\
\hline Minnesota........................ & 1.77 & 0 & 2,010 & 0 & 2,010 & 0 & 2,010 & 0.35 & - & 2,010 & 130 & 0 & 130 & 50,600 \\
\hline Mississippi ...................... & 33.7 & 6.35 & 84.0 & 2.07 & 118 & 8.42 & 126 & 5.41 & 0.01 & 132 & 36.1 & 6.01 & 42.1 & 57,500 \\
\hline Missouri …..................... & 13.8 & 0 & 5,840 & 0 & 5,860 & 0 & 5,860 & - & 1.64 & 5,860 & 90.6 & 0 & 90.6 & 79,700 \\
\hline Montana ......................... & 0.80 & 0 & 74.9 & 0 & 75.7 & 0 & 75.7 & - & - & 75.7 & 19.1 & 0 & 19.1 & 16,500 \\
\hline Nebraska ......................... & 16.8 & 0 & 2,900 & 0 & 2,920 & 0 & 2,920 & 0.04 & - & 2,920 & 30.7 & 0 & 30.7 & 34,300 \\
\hline Nevada ........................... & 7.04 & 70.9 & 1.69 & 0 & 8.73 & 70.9 & 79.7 & 0.56 & 0.49 & 80.7 & 4.63 & 27.5 & 32.1 & 11,400 \\
\hline New Hampshire ........... & 1.10 & 0 & 73.7 & 693 & 74.8 & 693 & 768 & 2.82 & 0.53 & 772 & 4.56 & 8.27 & 12.8 & 18,200 \\
\hline New Jersey ...................... & 2.08 & 0 & 358 & 3,430 & 361 & 3,430 & 3,790 & 10.7 & 0.71 & 3,800 & 20.5 & 76.0 & 96.5 & 63,700 \\
\hline New Mexico.................... & 6.13 & 0 & 27.3 & 0 & 33.5 & 0 & 33.5 & 0 & 0.26 & 33.7 & 27.7 & 0 & 27.7 & 25,000 \\
\hline New York ....................... & 7.30 & 0 & 2,200 & 5,470 & 2,210 & 5,470 & 7,680 & 2.76 & 17.1 & 7,700 & 67.0 & 54.8 & 122 & 89,400 \\
\hline North Carolina .............. & 0.18 & 0 & 6,180 & 1,360 & 6,180 & 1,360 & 7,540 & - & 8.33 & 7,550 & 113 & 13.6 & 127 & 113,000 \\
\hline North Dakota..................... & 0.42 & 0 & 983 & 0 & 983 & 0 & 983 & 1.13 & 0.05 & 984 & 28.6 & 0 & 28.6 & 34,300 \\
\hline Ohio................................. & 11.0 & 0 & 4,470 & 0 & 4,480 & 0 & 4,480 & - & 4.19 & 4,490 & 230 & 0 & 230 & 117,000 \\
\hline Oklahoma ........................ & 1.59 & 0 & 70.2 & 0 & 71.7 & 0 & 71.7 & 8.94 & 6.96 & 87.6 & 53.5 & 0 & 53.5 & 60,600 \\
\hline 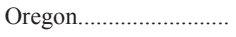 & 1.57 & 0 & 9.79 & 0 & 11.4 & 0 & 11.4 & 0 & 4.53 & 15.9 & 14.4 & 0 & 14.4 & 7,080 \\
\hline Pennsylvania .................... & 5.38 & 0 & 3,570 & 0 & 3,580 & 0 & 3,580 & 2.18 & - & 3,580 & 240 & 0 & 240 & 189,000 \\
\hline Rhode Island ..................... & 0 & 0 & 1.33 & 222 & 1.33 & 222 & 223 & - & 1.44 & 224 & 1.98 & 2.22 & 4.20 & 5,240 \\
\hline South Carolina .............. & 4.52 & 0 & 4,980 & 0 & 4,980 & 0 & 4,980 & 0 & 3.93 & 4,990 & 159 & 0 & 159 & 89,200 \\
\hline South Dakota.................... & 0 & 0 & 2.39 & 0 & 2.39 & 0 & 2.39 & - & - & 2.39 & 2.39 & 0 & 2.39 & 1,470 \\
\hline Tennessee ........................ & 2.18 & 0 & 4,620 & 0 & 4,620 & 0 & 4,620 & - & - & 4,620 & 62.8 & 0 & 62.8 & 62,600 \\
\hline 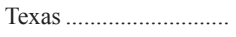 & 37.7 & 0 & 9,600 & 757 & 9,640 & 757 & 10,400 & 38.2 & 48.3 & 10,500 & 294 & 4.71 & 299 & 351,000 \\
\hline 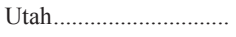 & 22.7 & 8.46 & 38.3 & 0 & 61.0 & 8.46 & 69.5 & 0.45 & 1.33 & 71.2 & 43.6 & 2.46 & 46.0 & 40,500 \\
\hline 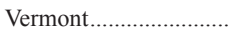 & 0.54 & 0 & 0.26 & 0 & 0.80 & 0 & 0.80 & - & - & 0.80 & 0.63 & 0 & 0.63 & 445 \\
\hline 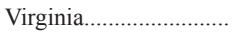 & 1.08 & 2.72 & 2,910 & 2,400 & 2,910 & 2,400 & 5,320 & 1.24 & 0.29 & 5,320 & 36.8 & 13.8 & 50.6 & 61,100 \\
\hline 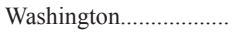 & 7.77 & 0 & 44.4 & 0 & 52.2 & 0 & 52.2 & - & 1.63 & 53.8 & 47.7 & 0 & 47.7 & 25,900 \\
\hline West Virginia.................... & 1.19 & 0 & 1,570 & 0 & 1,570 & 0 & 1,570 & - & 0.62 & 1,570 & 89.9 & 0 & 89.9 & 67,700 \\
\hline Wisconsin ......................... & 3.21 & 0 & 4,200 & 0 & 4,210 & 0 & 4,210 & 2.27 & 0.23 & 4,210 & 74.7 & 0 & 74.7 & 58,100 \\
\hline 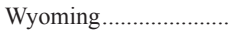 & 1.31 & 0 & 50.5 & 0 & 51.8 & 0 & 51.8 & - & - & 51.8 & 37.0 & 0 & 37.0 & 35,600 \\
\hline Puerto Rico..................... & 1.82 & 0 & 2.83 & 1,700 & 4.65 & 1,700 & 1,710 & 2.69 & 0.97 & 1,710 & 2.34 & 77.1 & 79.5 & 14,300 \\
\hline U.S. Virgin Islands ....... & 0 & 0 & 0.14 & 96.5 & 0.14 & 96.5 & 96.7 & - & - & 96.7 & 0 & 3.88 & 3.88 & 693 \\
\hline TOTAL & 425 & 172 & 94,700 & 37,600 & 95,100 & 37,800 & 133,000 & 203 & 220 & 133,000 & 3,760 & 547 & 4,310 & $3,230,000$ \\
\hline
\end{tabular}




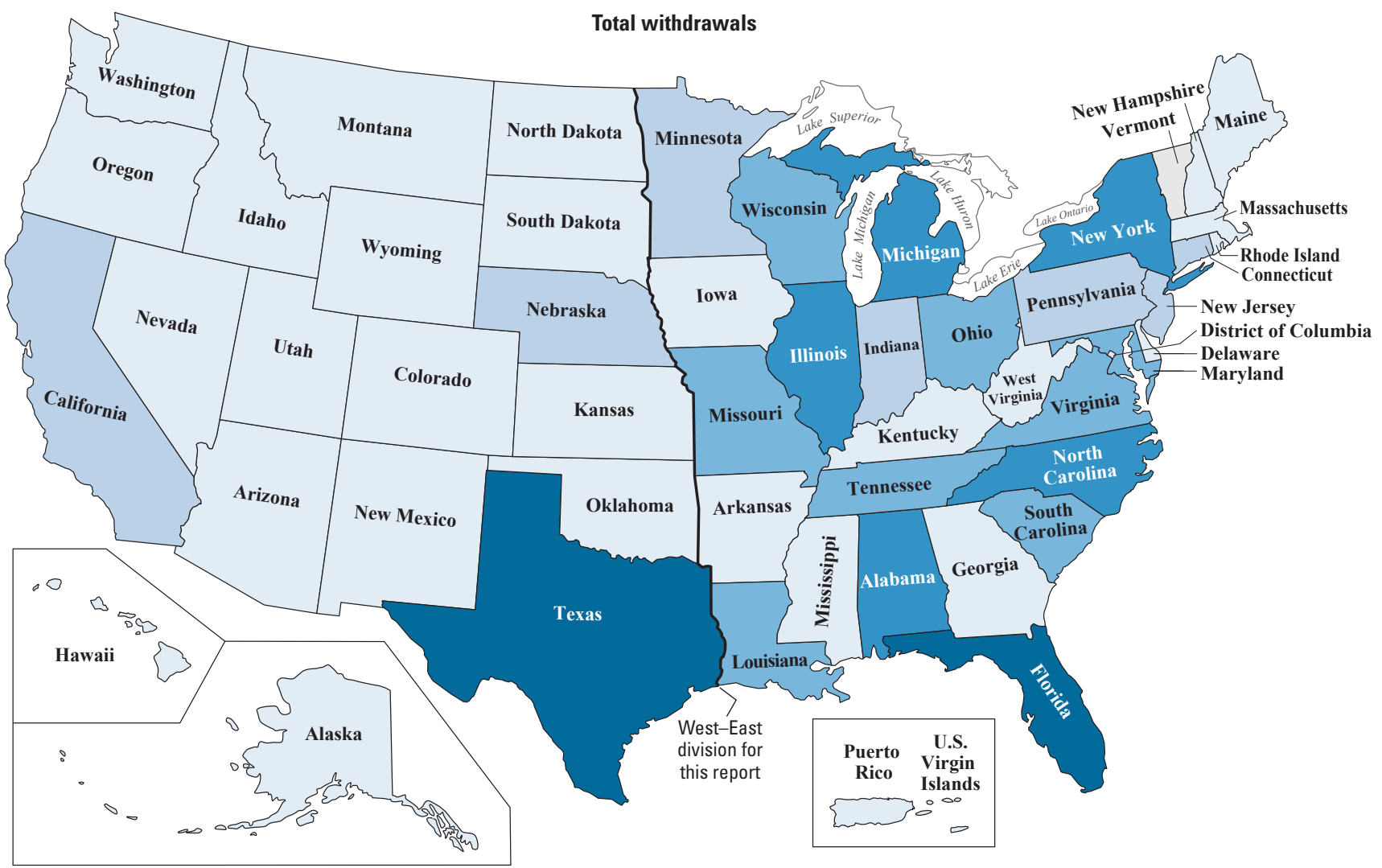

EXPLANATION

Water withdrawals, in million gallons per day
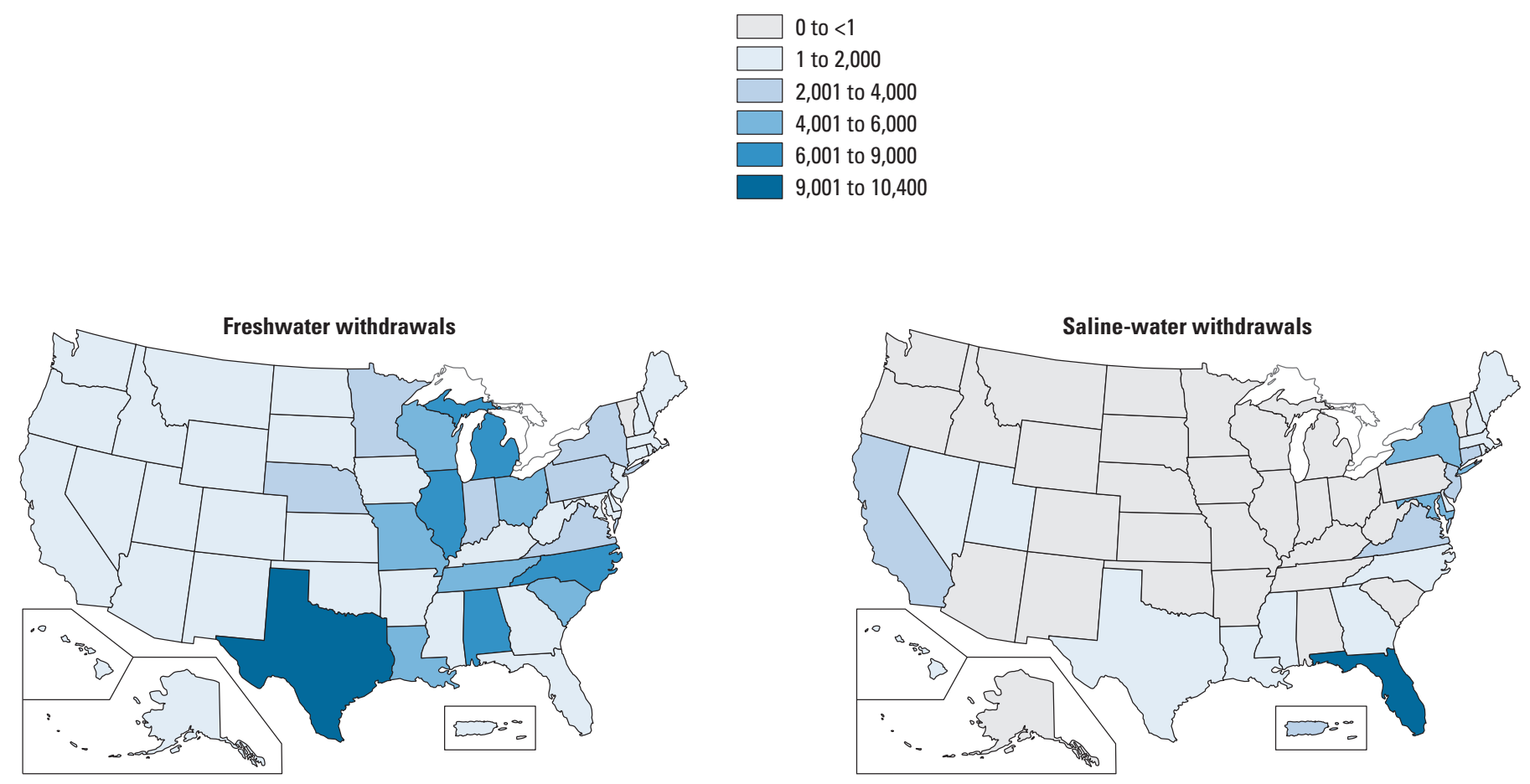

Figure 13. Thermoelectric-power withdrawals by water quality and State, 2015. 
United States in 2015. Hydroelectric-power generation is not included in this report, but accounts for about 6 percent of the United States total energy needs and is an important energy source in the Western United States (U.S. Department of Energy, 2016b). In 2015, 58 percent of the total $248,000 \mathrm{gWh}$ from hydroelectric powerplants was produced by utilities in Washington, California, Oregon, and New York (U.S. Department of Energy, 2016b).

The geographic distribution of total water use and total consumptive use is shown in figure $14 A$ for once-through cooling systems and figure $14 B$ for recirculating cooling systems. Powerplants equipped with once-through cooling systems accounted for 96 percent of total thermoelectricpower withdrawals and 37 percent of net power generated (table $13 \mathrm{~A}$ ), whereas plants with recirculating cooling systems withdrew much less water (4 percent of total withdrawals) and produced most (63 percent) of the power (table 13B). Plants with recirculating cooling systems accounted for 67 percent of total consumptive use. Consumptive use for recirculating cooling systems was 57 percent of total recirculating withdrawals, whereas consumptive use for once-through systems was just 1 percent of total once-through withdrawals (tables $13 A$ and $13 B$ ). Powerplants with recirculating cooling systems are found in every State except the District of Columbia and the U.S. Virgin Islands. Powerplants with once-through cooling systems are found in every State except Arizona, Colorado,
District of Columbia, Idaho, Nevada, New Mexico, Oklahoma, Oregon, South Dakota, Utah, Vermont, Washington, and Wyoming.

Reporting of public-supply deliveries and the use of reclaimed wastewater for cooling purposes was not required for 2015, although values were estimated for some States (table 12; fig. 14A and $B$ ). Public-supply deliveries were reported for 32 States and totaled $220 \mathrm{Mgal} / \mathrm{d}$, less than 1 percent of total thermoelectric withdrawals. Texas (48.3 Mgal/d), Massachusetts (47.0 Mgal/d), and California (38.9 Mgal/d) accounted for 61 percent of total public-supply deliveries to thermoelectric plants. Reclaimed wastewater was reported for 25 States and totaled $203 \mathrm{Mgal} / \mathrm{d}$. Arizona reported the largest use of reclaimed wastewater at $67.7 \mathrm{Mgal} / \mathrm{d}$, almost all of which was used at the Palo Verde nuclear powerplant in Maricopa County, Arizona. Texas (38.2 Mgal/d) and Florida (34.5 Mgal/d) reported substantial amounts of reclaimed wastewater use as well. Plants with recirculating cooling systems accounted for 77 percent of public-supply deliveries and 95 percent of reclaimed wastewater uses (fig. 14B). Note that the reported public-supply deliveries and reclaimed wastewater data, because the reporting of data was not a requirement for this compilation, may not include all instances of these types of water use and, therefore, may be underestimated.

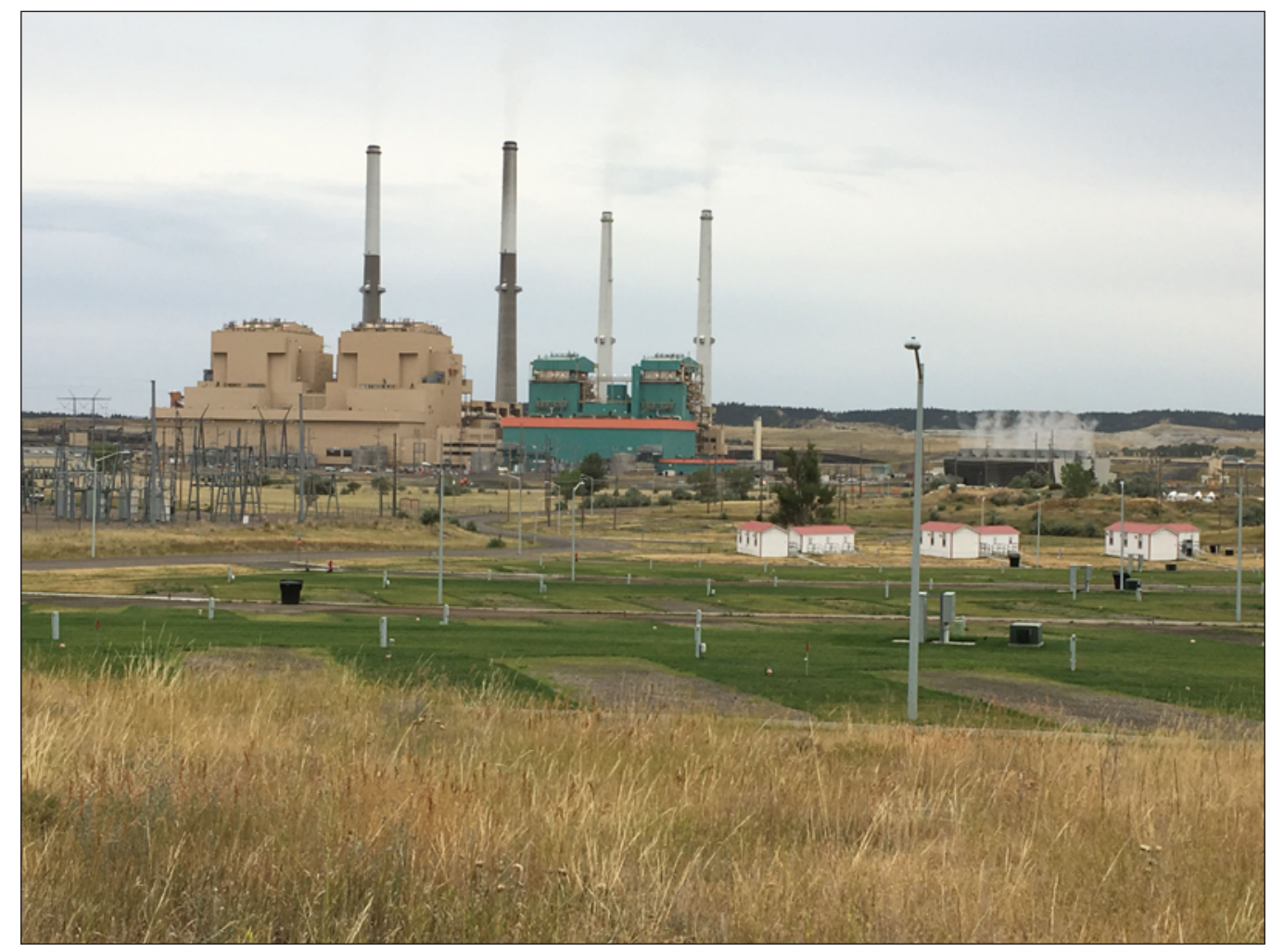

Thermoelectric powerplant in Montana. Photograph by Rodney Caldwell, USGS. 

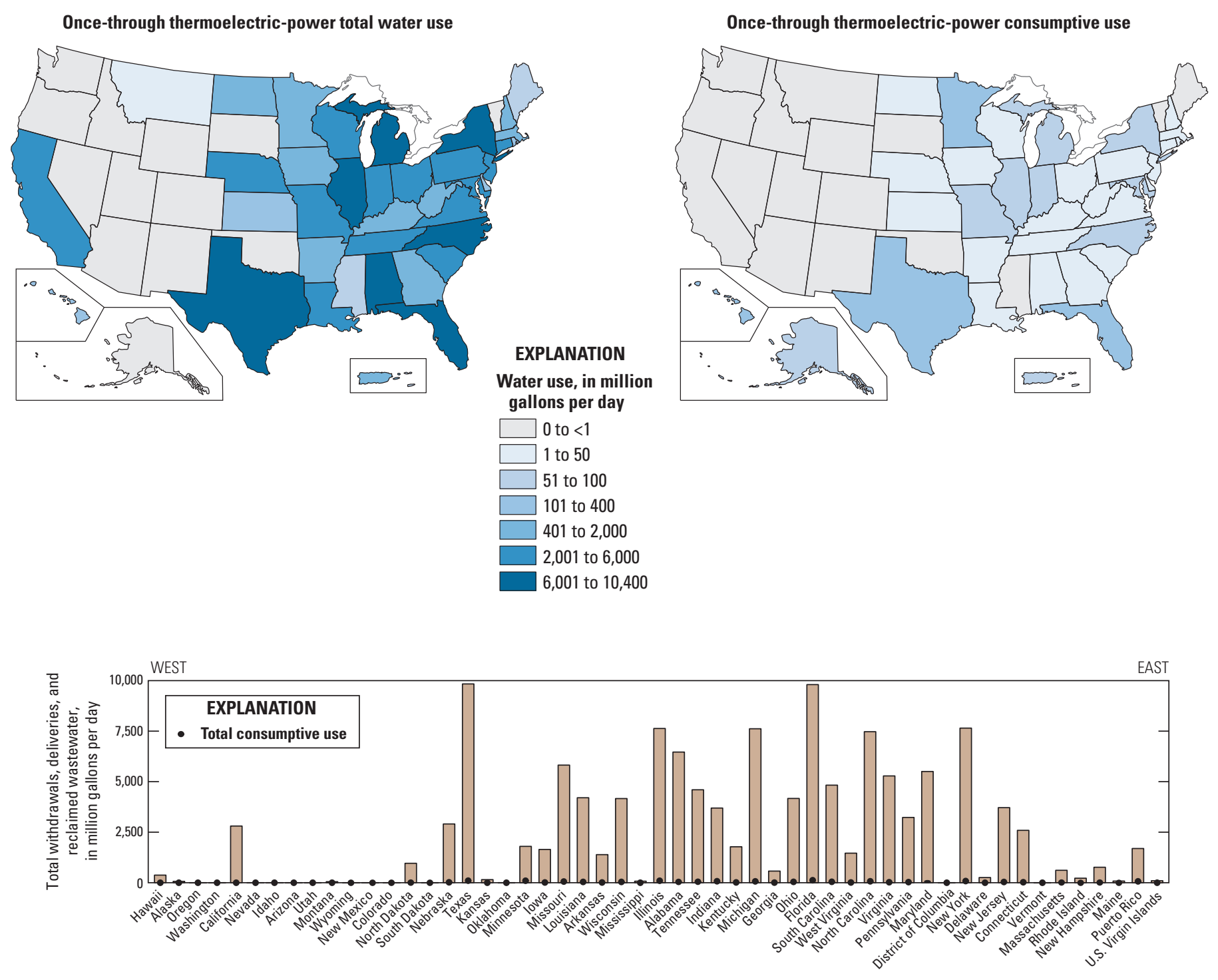

Figure 14A. Thermoelectric-power water use and consumptive use by State for once-through cooling types, 2015. 

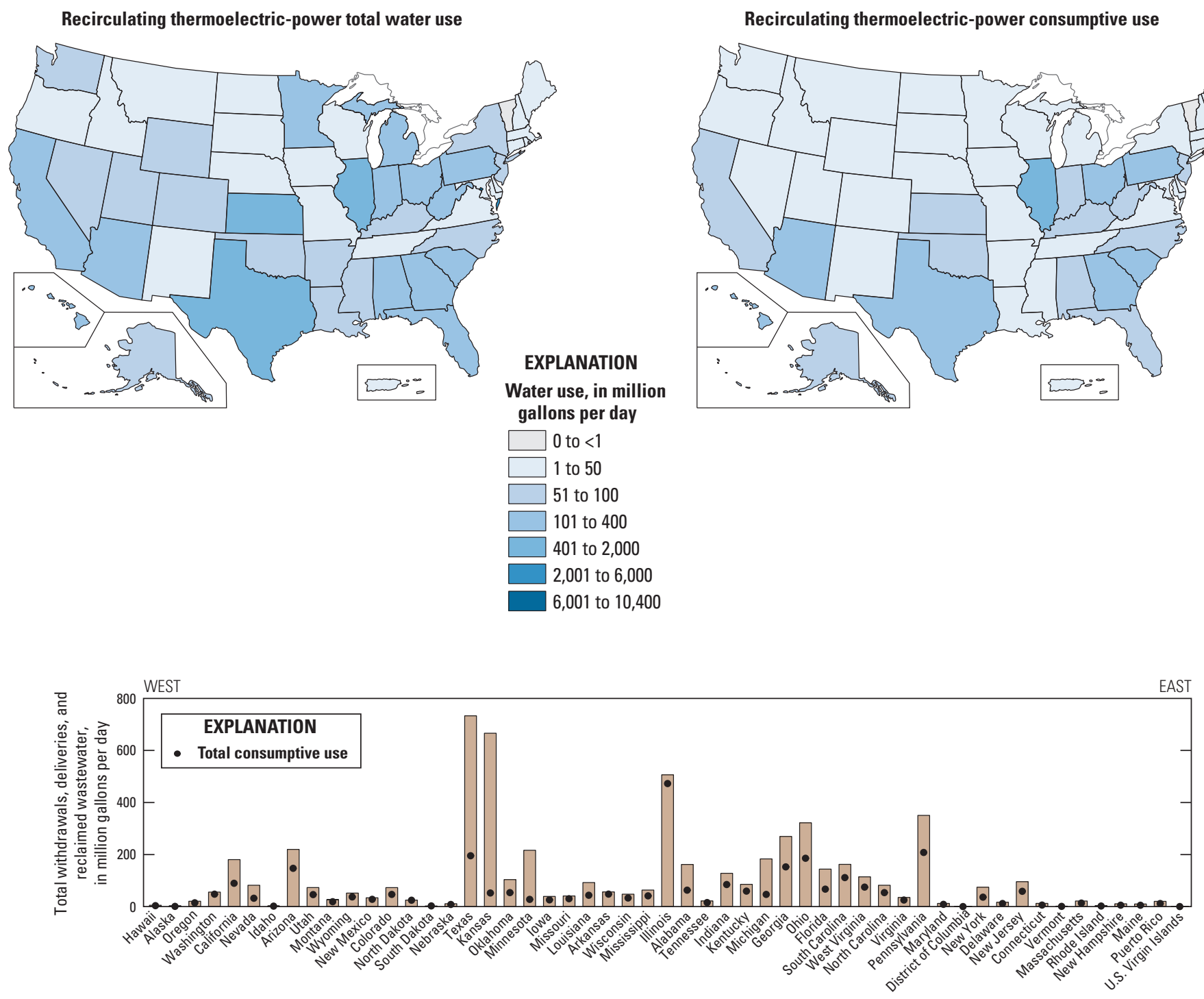

Figure 14B. Thermoelectric-power water use and consumptive use by State for recirculating cooling types, 2015. 
Table 13A. Once-through cooling thermoelectric-power water use, 2015.

[Values may not sum to totals because of independent rounding. All withdrawal, reclaimed wastewater, delivery, and use data are in million gallons per day. Abbreviation: - , not estimated]

\begin{tabular}{|c|c|c|c|c|c|c|c|c|c|c|c|c|c|c|}
\hline \multirow{4}{*}{ State } & \multicolumn{7}{|c|}{ Withdrawals for once-through cooling } & \multirow{4}{*}{$\begin{array}{c}\text { Re- } \\
\text { claimed } \\
\text { waste- } \\
\text { water }\end{array}$} & \multirow{4}{*}{$\begin{array}{c}\text { Public- } \\
\text { supply } \\
\text { deliveries } \\
\text { to once- } \\
\text { through } \\
\text { cooling } \\
\text { thermoelec- } \\
\text { tric-power } \\
\text { generation } \\
\text { use } \\
\text { Fresh }\end{array}$} & \multicolumn{4}{|c|}{ Total use } & \multirow{4}{*}{$\begin{array}{c}\text { Net power } \\
\text { generated } \\
\text { (gigawatt- } \\
\text { hours) }\end{array}$} \\
\hline & \multirow{2}{*}{\multicolumn{2}{|c|}{ Groundwater }} & \multirow{2}{*}{\multicolumn{2}{|c|}{ Surface water }} & \multirow{2}{*}{\multicolumn{3}{|c|}{ Total }} & & & \multirow{3}{*}{$\begin{array}{c}\text { Water use } \\
\text { (with- } \\
\text { drawals, } \\
\text { deliver- } \\
\text { ies, and } \\
\text { reclaimed } \\
\text { waste- } \\
\text { water) } \\
\text { Total }\end{array}$} & \multirow{2}{*}{\multicolumn{3}{|c|}{ Consumptive use }} & \\
\hline & & & & & & & & & & & & & & \\
\hline & Fresh & Saline & Fresh & Saline & Fresh & Saline & Total & & & & Fresh & Saline & Total & \\
\hline Alabama ............................. & 0 & 0 & 6,460 & 0 & 6,460 & 0 & 6,460 & - & - & 6,460 & 44.1 & 0 & 44.1 & 44,900 \\
\hline Alaska .............................. & 0 & 0 & 66.1 & 0 & 66.1 & 0 & 66.1 & - & - & 66.1 & 0.50 & 0 & 0.50 & 446 \\
\hline 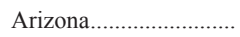 & 0 & 0 & 0 & 0 & 0 & 0 & 0 & 0 & 0 & 0 & 0 & 0 & 0 & 0 \\
\hline 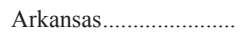 & 0 & 0 & 1,390 & 0 & 1,390 & 0 & 1,390 & - & - & 1,390 & 13.3 & 0 & 13.3 & 9,910 \\
\hline 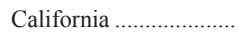 & 0 & 0 & 0 & 2,800 & 0 & 2,800 & 2,800 & 0 & 0 & 2,800 & 0 & 0.19 & 0.19 & 30,300 \\
\hline 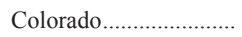 & 0 & 0 & 0 & 0 & 0 & 0 & 0 & - & - & 0 & 0 & 0 & 0 & 0 \\
\hline Connecticut ..................... & 0 & 0 & 123 & 2,470 & 123 & 2,470 & 2,590 & - & - & 2,590 & 1.16 & 23.2 & 24.4 & 21,900 \\
\hline 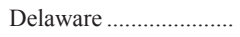 & 0 & 0 & 0 & 254 & 0 & 254 & 254 & - & - & 254 & 0 & 2.54 & 2.54 & 586 \\
\hline District of Columbia ... & 0 & 0 & 0 & 0 & 0 & 0 & 0 & 0 & 0 & 0 & 0 & 0 & 0 & 0 \\
\hline 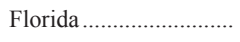 & 2.17 & 27.5 & 354 & 9,400 & 356 & 9,420 & 9,780 & 7.10 & 3.81 & 9,790 & 5.26 & 121 & 127 & 93,900 \\
\hline 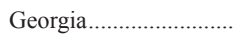 & 0.91 & 0 & 471 & 102 & 472 & 102 & 574 & - & - & 574 & 8.16 & 0.02 & 8.18 & 2,540 \\
\hline 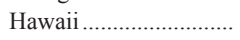 & 0.78 & 15.9 & 0 & 357 & 0.78 & 373 & 374 & - & - & 374 & 0.01 & 0 & 0.01 & 4,120 \\
\hline 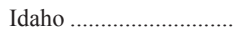 & 0 & 0 & 0 & 0 & 0 & 0 & 0 & - & - & 0 & 0 & 0 & 0 & 0 \\
\hline 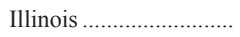 & 1.98 & 0 & 7,630 & 0 & 7,630 & 0 & 7,630 & - & - & 7,630 & 99.3 & 0 & 99.3 & 79,900 \\
\hline 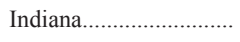 & 13.9 & 0 & 3,680 & 0 & 3,690 & 0 & 3,690 & - & - & 3,690 & 76.3 & 0 & 76.3 & 30,000 \\
\hline 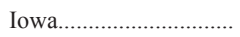 & 5.49 & 0 & 1,640 & 0 & 1,650 & 0 & 1,650 & - & - & 1,650 & 14.2 & 0 & 14.2 & 20,000 \\
\hline 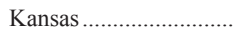 & 0.06 & 0 & 150 & 0 & 151 & 0 & 151 & - & - & 151 & 1.69 & 0 & 1.69 & 1,520 \\
\hline 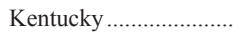 & 0 & 0 & 1,780 & 0 & 1,780 & 0 & 1,780 & - & - & 1,780 & 17.7 & 0 & 17.7 & 28,900 \\
\hline Louisiana.......................... & 24.6 & 0 & 3,920 & 261 & 3,940 & 261 & 4,200 & - & - & 4,200 & 38.9 & 2.61 & 41.5 & 39,700 \\
\hline 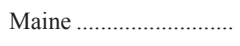 & 0 & 0 & 0 & 80.4 & 0 & 80.4 & 80.4 & - & - & 80.4 & 0 & 0.81 & 0.81 & 573 \\
\hline 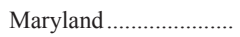 & 2.21 & 0 & 218 & 5,280 & 220 & 5,280 & 5,500 & - & - & 5,500 & 2.19 & 52.8 & 55.0 & 22,800 \\
\hline Massachusetts .............. & 0 & 0 & 46.9 & 473 & 46.9 & 473 & 520 & 0 & 45.7 & 566 & 0.94 & 4.74 & 5.68 & 9,060 \\
\hline Michigan .......................... & 1.33 & 0 & 7,620 & 0 & 7,620 & 0 & 7,620 & - & - & 7,620 & 70.2 & 0 & 70.2 & 72,600 \\
\hline 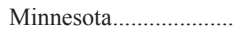 & 0.43 & 0 & 1,800 & 0 & 1,800 & 0 & 1,800 & 0 & - & 1,800 & 103 & 0 & 103 & 42,200 \\
\hline Mississippi ...................... & 0 & 0 & 73.7 & 0 & 73.7 & 0 & 73.7 & 0 & 0 & 73.7 & 0.87 & 0 & 0.87 & 601 \\
\hline Missouri ............................ & 0 & 0 & 5,820 & 0 & 5,820 & 0 & 5,820 & - & - & 5,820 & 60.5 & 0 & 60.5 & 62,900 \\
\hline Montana ........................... & 0 & 0 & 49.2 & 0 & 49.2 & 0 & 49.2 & - & - & 49.2 & 0.49 & 0 & 0.49 & 375 \\
\hline Nebraska .......................... & 11.1 & 0 & 2,900 & 0 & 2,910 & 0 & 2,910 & - & - & 2,910 & 22.7 & 0 & 22.7 & 27,000 \\
\hline Nevada ............................ & 0 & 0 & 0 & 0 & 0 & 0 & 0 & - & - & 0 & 0 & 0 & 0 & 0 \\
\hline New Hampshire ........... & 0.20 & 0 & 72.0 & 692 & 72.2 & 692 & 764 & 0.14 & 0.18 & 764 & 0.54 & 6.92 & 7.46 & 10,900 \\
\hline New Jersey ...................... & 0 & 0 & 348 & 3,370 & 348 & 3,370 & 3,710 & 0 & 0 & 3,710 & 3.54 & 34.2 & 37.8 & 24,700 \\
\hline New Mexico.................. & 0 & 0 & 0 & 0 & 0 & 0 & 0 & 0 & 0 & 0 & 0 & 0 & 0 & 0 \\
\hline New York ......................... & 0 & 0 & 2,180 & 5,470 & 2,180 & 5,470 & 7,650 & 0 & 0 & 7,650 & 30.7 & 54.8 & 85.5 & 58,800 \\
\hline North Carolina .............. & 0 & 0 & 6,120 & 1,360 & 6,120 & 1,360 & 7,470 & 0 & 0 & 7,470 & 59.6 & 13.6 & 73.1 & 66,600 \\
\hline North Dakota................ & 0 & 0 & 959 & 0 & 959 & 0 & 959 & 1.00 & 0.05 & 960 & 4.10 & 0 & 4.10 & 15,300 \\
\hline 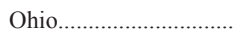 & 4.36 & 0 & 4,160 & 0 & 4,170 & 0 & 4,170 & - & 0.75 & 4,170 & 43.9 & 0 & 43.9 & 32,400 \\
\hline 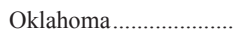 & 0 & 0 & 0 & 0 & 0 & 0 & 0 & 0 & 0 & 0 & 0 & 0 & 0 & 0 \\
\hline 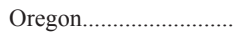 & 0 & 0 & 0 & 0 & 0 & 0 & 0 & 0 & 0 & 0 & 0 & 0 & 0 & 0 \\
\hline Pennsylvania ................... & 0.60 & 0 & 3,230 & 0 & 3,230 & 0 & 3,230 & 1.12 & - & 3,230 & 32.5 & 0 & 32.5 & 35,000 \\
\hline Rhode Island ................... & 0 & 0 & 0 & 222 & 0 & 222 & 222 & - & - & 222 & 0 & 2.22 & 2.22 & 1,910 \\
\hline South Carolina .............. & 0 & 0 & 4,830 & 0 & 4,830 & 0 & 4,830 & 0 & 0 & 4,830 & 48.0 & 0 & 48.0 & 43,400 \\
\hline South Dakota................. & 0 & 0 & 0 & 0 & 0 & 0 & 0 & - & - & 0 & 0 & 0 & 0 & 0 \\
\hline Tennessee ........................... & 0 & 0 & 4,600 & 0 & 4,600 & 0 & 4,600 & - & - & 4,600 & 47.3 & 0 & 47.3 & 46,200 \\
\hline 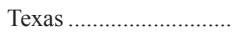 & 9.82 & 0 & 9,450 & 379 & 9,460 & 379 & 9,840 & - & 0.04 & 9,840 & 104 & 0 & 104 & 111,000 \\
\hline 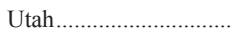 & 0 & 0 & 0 & 0 & 0 & 0 & 0 & - & - & 0 & 0 & 0 & 0 & 0 \\
\hline Vermont........................... & 0 & 0 & 0 & 0 & 0 & 0 & 0 & - & - & 0 & 0 & 0 & 0 & 0 \\
\hline Virginia............................ & 0.35 & 0 & 2,890 & 2,400 & 2,890 & 2,400 & 5,280 & - & - & 5,280 & 14.4 & 11.2 & 25.6 & 38,900 \\
\hline 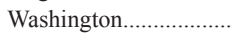 & 0 & 0 & 0 & 0 & 0 & 0 & 0 & - & 0 & 0 & 0 & 0 & 0 & 0 \\
\hline 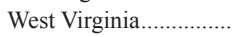 & 0.03 & 0 & 1,460 & 0 & 1,460 & 0 & 1,460 & - & 0.03 & 1,460 & 14.9 & 0 & 14.9 & 11,700 \\
\hline Wisconsin ......................... & 0.49 & 0 & 4,160 & 0 & 4,160 & 0 & 4,160 & 0 & 0 & 4,160 & 41.6 & 0 & 41.6 & 36,600 \\
\hline 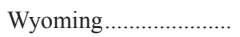 & 0 & 0 & 0 & 0 & 0 & 0 & 0 & - & - & 0 & 0 & 0 & 0 & 0 \\
\hline Puerto Rico....................... & 1.63 & 0 & 0.24 & 1,690 & 1.87 & 1,690 & 1,690 & 0 & 0.97 & 1,690 & 0.11 & 67.6 & 67.7 & 8,100 \\
\hline U.S. Virgin Islands ....... & 0 & 0 & 0.14 & 96.5 & 0.14 & 96.5 & 96.7 & - & - & 96.7 & 0 & 3.88 & 3.88 & 693 \\
\hline TOTAL & 82.4 & 43.4 & 90,600 & 37,200 & 90,700 & 37,200 & 128,000 & 9.36 & 51.5 & 128,000 & 1,030 & 403 & 1,430 & $1,190,000$ \\
\hline
\end{tabular}


Table 13B. Recirculating cooling thermoelectric-power water use, 2015.

[Values may not sum to totals because of independent rounding. All withdrawal, reclaimed wastewater, delivery, and use data are in million gallons per day. Abbreviation: - , not estimated]

\begin{tabular}{|c|c|c|c|c|c|c|c|c|c|c|c|c|c|c|}
\hline \multirow{4}{*}{ State } & \multicolumn{7}{|c|}{ Withdrawals for recirculating cooling } & \multirow{4}{*}{$\begin{array}{c}\text { Re- } \\
\text { claimed } \\
\text { waste- } \\
\text { water }\end{array}$} & \multirow{4}{*}{$\begin{array}{c}\text { Public- } \\
\text { supply } \\
\text { deliveries } \\
\text { to recir- } \\
\text { culating } \\
\text { cooling } \\
\text { thermoelec- } \\
\text { tric-power } \\
\text { generation } \\
\text { use } \\
\text { Fresh }\end{array}$} & \multicolumn{4}{|c|}{ Total use } & \multirow{4}{*}{$\begin{array}{c}\text { Net power } \\
\text { generated } \\
\text { (gigawatt- } \\
\text { hours) }\end{array}$} \\
\hline & \multirow{2}{*}{\multicolumn{2}{|c|}{ Groundwater }} & \multirow{2}{*}{\multicolumn{2}{|c|}{ Surface water }} & \multirow{2}{*}{\multicolumn{3}{|c|}{ Total }} & & & \multirow{3}{*}{$\begin{array}{c}\text { Water use } \\
\text { (with- } \\
\text { drawals, } \\
\text { deliver- } \\
\text { ies, and } \\
\text { reclaimed } \\
\text { waste- } \\
\text { water) } \\
\text { Total }\end{array}$} & \multirow{2}{*}{\multicolumn{3}{|c|}{ Consumptive use }} & \\
\hline & & & & & & & & & & & & & & \\
\hline & Fresh & Saline & Fresh & Saline & Fresh & Saline & Total & & & & Fresh & Saline & Total & \\
\hline 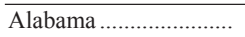 & 0 & 0 & 162 & 0 & 162 & 0 & 162 & - & - & 162 & 63.1 & 0 & 63.1 & 82,700 \\
\hline 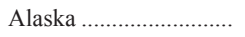 & 0.63 & 0 & 0 & 0 & 0.63 & 0 & 0.63 & - & 0.60 & 1.23 & 0.88 & 0 & 0.88 & 1,430 \\
\hline 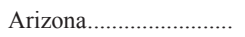 & 57.7 & 0 & 25.8 & 0 & 83.5 & 0 & 83.5 & 67.7 & 0.50 & 152 & 147 & 0 & 147 & 102,000 \\
\hline 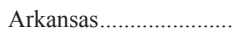 & 3.13 & 0 & 53.2 & 0 & 56.3 & 0 & 56.3 & - & - & 56.3 & 47.9 & 0 & 47.9 & 39,600 \\
\hline California ...................... & 19.6 & 35.1 & 16.7 & 0.05 & 36.4 & 35.2 & 71.5 & 15.6 & 38.9 & 126 & 64.4 & 25.1 & 89.5 & 80,600 \\
\hline Colorado............................ & 3.03 & 0 & 34.1 & 0 & 37.2 & 0 & 37.2 & 4.11 & 13.7 & 55.0 & 46.7 & 0 & 46.7 & 42,300 \\
\hline Connecticut ..................... & 0 & 0 & 2.96 & 0 & 2.96 & 0 & 2.96 & - & 4.65 & 7.61 & 5.89 & 0 & 5.89 & 9,040 \\
\hline Delaware .......................... & 0.13 & 0 & 14.3 & 2.12 & 14.4 & 2.12 & 16.5 & - & - & 16.5 & 10.3 & 1.48 & 11.8 & 5,080 \\
\hline District of Columbia ... & 0 & 0 & 0 & 0 & 0 & 0 & 0 & 0 & 0 & 0 & 0 & 0 & 0 & 0 \\
\hline Florida ............................ & 25.7 & 1.06 & 52.2 & 0 & 77.8 & 1.06 & 78.9 & 27.4 & 5.07 & 111 & 66.3 & 0.74 & 67.0 & 137,000 \\
\hline 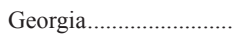 & 2.52 & 0 & 267 & 0 & 270 & 0 & 270 & - & - & 270 & 153 & 0 & 153 & 108,000 \\
\hline 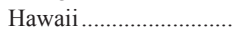 & 0.70 & 4.34 & 0 & 0 & 0.70 & 4.34 & 5.04 & - & - & 5.04 & 0.50 & 3.10 & 3.60 & 3,700 \\
\hline 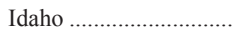 & 1.79 & 0 & 0 & 0 & 1.79 & 0 & 1.79 & - & - & 1.79 & 1.79 & 0 & 1.79 & 2,210 \\
\hline 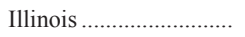 & 2.23 & 0 & 504 & 0 & 506 & 0 & 506 & - & - & 506 & 473 & 0 & 473 & 97,100 \\
\hline Indiana............................. & 6.93 & 0 & 121 & 0 & 128 & 0 & 128 & - & - & 128 & 84.7 & 0 & 84.7 & 57,000 \\
\hline 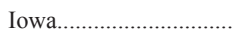 & 21.5 & 0 & 16.4 & 0 & 37.8 & 0 & 37.8 & 0.42 & 0.16 & 38.4 & 25.8 & 0 & 25.8 & 15,600 \\
\hline 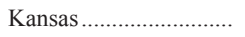 & 7.75 & 0 & 658 & 0 & 666 & 0 & 666 & - & 0.25 & 666 & 52.4 & 0 & 52.4 & 32,200 \\
\hline Kentucky ......................... & 4.96 & 0 & 80.5 & 0 & 85.5 & 0 & 85.5 & - & - & 85.5 & 59.4 & 0 & 59.4 & 45,800 \\
\hline Louisiana......................... & 12.4 & 0 & 79.8 & 0 & 92.3 & 0 & 92.3 & - & - & 92.3 & 43.4 & 0 & 43.4 & 30,000 \\
\hline Maine ............................. & 0.92 & 0 & 4.38 & 0.27 & 5.30 & 0.27 & 5.57 & - & 2.05 & 7.62 & 4.94 & 0.19 & 5.13 & 4,390 \\
\hline 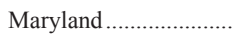 & 0.09 & 0 & 0.14 & 11.9 & 0.23 & 11.9 & 12.1 & - & - & 12.1 & 0.10 & 8.31 & 8.41 & 7,940 \\
\hline Massachusetts .............. & 1.10 & 0 & 2.13 & 13.5 & 3.23 & 13.5 & 16.7 & 1.07 & 1.30 & 19.1 & 3.92 & 9.46 & 13.4 & 7,760 \\
\hline Michigan ........................... & 4.28 & 0 & 179 & 0 & 183 & 0 & 183 & - & - & 183 & 46.9 & 0 & 46.9 & 29,500 \\
\hline Minnesota.......................... & 1.34 & 0 & 215 & 0 & 216 & 0 & 216 & 0.35 & - & 216 & 27.6 & 0 & 27.6 & 8,420 \\
\hline Mississippi .................... & 33.7 & 6.35 & 10.3 & 2.07 & 44.0 & 8.42 & 52.5 & 5.41 & 0.01 & 57.9 & 35.2 & 6.01 & 41.2 & 56,900 \\
\hline 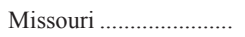 & 13.8 & 0 & 23.7 & 0 & 37.4 & 0 & 37.4 & - & 1.64 & 39.1 & 30.1 & 0 & 30.1 & 16,900 \\
\hline Montana ........................... & 0.80 & 0 & 25.7 & 0 & 26.5 & 0 & 26.5 & - & - & 26.5 & 18.6 & 0 & 18.6 & 16,100 \\
\hline Nebraska .......................... & 5.71 & 0 & 5.16 & 0 & 10.9 & 0 & 10.9 & 0.04 & - & 10.9 & 7.99 & 0 & 7.99 & 7,320 \\
\hline Nevada ............................ & 7.04 & 70.9 & 1.69 & 0 & 8.73 & 70.9 & 79.7 & 0.56 & 0.49 & 80.7 & 4.63 & 27.5 & 32.1 & 11,400 \\
\hline New Hampshire ........... & 0.90 & 0 & 1.67 & 1.83 & 2.57 & 1.83 & 4.40 & 2.68 & 0.35 & 7.43 & 4.02 & 1.35 & 5.37 & 7,330 \\
\hline New Jersey ...................... & 2.08 & 0 & 10.8 & 59.8 & 12.9 & 59.8 & 72.6 & 10.7 & 0.71 & 84.1 & 16.9 & 41.8 & 58.7 & 39,000 \\
\hline New Mexico................. & 6.13 & 0 & 27.3 & 0 & 33.5 & 0 & 33.5 & 0 & 0.26 & 33.7 & 27.7 & 0 & 27.7 & 25,000 \\
\hline New York ......................... & 7.30 & 0 & 27.2 & 0 & 34.6 & 0 & 34.6 & 2.76 & 17.1 & 54.4 & 36.3 & 0 & 36.3 & 30,600 \\
\hline North Carolina .............. & 0.18 & 0 & 65.2 & 0 & 65.4 & 0 & 65.4 & - & 8.33 & 73.8 & 53.5 & 0 & 53.5 & 46,000 \\
\hline North Dakota................ & 0.42 & 0 & 24.3 & 0 & 24.7 & 0 & 24.7 & 0.13 & 0 & 24.8 & 24.5 & 0 & 24.5 & 19,000 \\
\hline 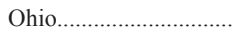 & 6.63 & 0 & 308 & 0 & 315 & 0 & 315 & - & 3.44 & 319 & 186 & 0 & 186 & 84,600 \\
\hline Oklahoma.......................... & 1.59 & 0 & 70.2 & 0 & 71.7 & 0 & 71.7 & 8.94 & 6.96 & 87.6 & 53.5 & 0 & 53.5 & 60,600 \\
\hline 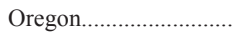 & 1.57 & 0 & 9.79 & 0 & 11.4 & 0 & 11.4 & 0 & 4.53 & 15.9 & 14.4 & 0 & 14.4 & 7,080 \\
\hline Pennsylvania ................... & 4.78 & 0 & 345 & 0 & 349 & 0 & 349 & 1.06 & - & 351 & 208 & 0 & 208 & 154,000 \\
\hline Rhode Island ................... & 0 & 0 & 1.33 & 0 & 1.33 & 0 & 1.33 & 0 & 1.44 & 2.77 & 1.98 & 0 & 1.98 & 3,330 \\
\hline South Carolina .............. & 4.52 & 0 & 150 & 0 & 155 & 0 & 155 & 0 & 3.93 & 158 & 111 & 0 & 111 & 45,800 \\
\hline South Dakota................. & 0 & 0 & 2.39 & 0 & 2.39 & 0 & 2.39 & - & - & 2.39 & 2.39 & 0 & 2.39 & 1,470 \\
\hline Tennessee ........................... & 2.18 & 0 & 19.4 & 0 & 21.6 & 0 & 21.6 & - & - & 21.6 & 15.4 & 0 & 15.4 & 16,400 \\
\hline 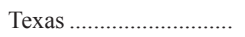 & 27.9 & 0 & 155 & 378 & 182 & 378 & 561 & 38.2 & 48.2 & 647 & 191 & 4.71 & 195 & 239,000 \\
\hline 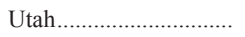 & 22.7 & 8.46 & 38.3 & 0 & 61.0 & 8.46 & 69.5 & 0.45 & 1.33 & 71.2 & 43.6 & 2.46 & 46.0 & 40,500 \\
\hline Vermont........................... & 0.54 & 0 & 0.26 & 0 & 0.80 & 0 & 0.80 & - & - & 0.80 & 0.63 & 0 & 0.63 & 445 \\
\hline 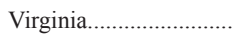 & 0.73 & 2.72 & 27.5 & 0.80 & 28.3 & 3.52 & 31.8 & 1.24 & 0.29 & 33.3 & 22.4 & 2.70 & 25.1 & 22,300 \\
\hline Washington....................... & 7.77 & 0 & 44.4 & 0 & 52.2 & 0 & 52.2 & - & 1.63 & 53.8 & 47.7 & 0 & 47.7 & 25,900 \\
\hline West Virginia..................... & 1.16 & 0 & 112 & 0 & 113 & 0 & 113 & - & 0.59 & 114 & 75.0 & 0 & 75.0 & 56,100 \\
\hline Wisconsin ........................ & 2.72 & 0 & 39.3 & 0 & 42.0 & 0 & 42.0 & 2.27 & 0.23 & 44.5 & 33.1 & 0 & 33.1 & 21,500 \\
\hline 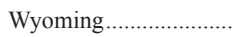 & 1.31 & 0 & 50.5 & 0 & 51.8 & 0 & 51.8 & - & - & 51.8 & 37.0 & 0 & 37.0 & 35,600 \\
\hline Puerto Rico....................... & 0.19 & 0 & 2.59 & 12.0 & 2.78 & 12.0 & 14.8 & 2.69 & - & 17.4 & 2.23 & 9.58 & 11.8 & 6,200 \\
\hline U.S. Virgin Islands ...... & 0 & 0 & 0 & 0 & 0 & 0 & 0 & - & - & 0 & 0 & 0 & 0 & 0 \\
\hline TOTAL & 343 & 129 & 4,090 & 482 & 4,430 & 611 & 5,040 & 194 & 169 & 5,400 & 2,730 & 144 & 2,880 & $2,050,000$ \\
\hline
\end{tabular}




\section{Trends in Water Use, 1950-2015}

The USGS has conducted water-use compilations every 5 years since 1950 (https://water.usgs.gov/watuse/50years. $\mathrm{html}$ ). A summary of population growth and withdrawal estimates by category of use and source of water is discussed in this section and shown in table 14 for each 5 -year period from 1950 through 2015. These trends are shown graphically for freshwater uses in figure 15 and total uses in figure 16.

Some categories have been compiled and presented differently since compilations were begun. For example, self-supplied domestic and livestock withdrawals are shown separately in table 14; however, they were combined as "rural" in the 1950 and 1955 reports. Prior to 1985, the industrial water-use category included withdrawals for commercial, mining, and aquaculture; after 1985 these categories were estimated separately. Water use at fish hatcheries was reported as commercial use in 1990 and 1995 but has been included in the aquaculture category since 2000. Estimates of commercial withdrawals have not been compiled nationally since
2000. Totals in table 14 represent the most current data and incorporate revisions to previously published data; therefore, percentage differences and national totals may be slightly different from those in previous reports (Solley and others, 1988, 1993, 1998; Hutson and others, 2004; Kenny and others, 2009; Maupin and others, 2014).

Some revisions at the county or State levels have been made to previously published data since Maupin and others (2014) was published. The revisions have resulted in changes in total withdrawals by category as noted in table 14. For example, mining withdrawals published in Maupin and others (2014; table 14) were $5.32 \mathrm{Bgal} / \mathrm{d}$, which is $1.35 \mathrm{Bgal} / \mathrm{d}$ more than the revised estimate of $3.97 \mathrm{Bgal} / \mathrm{d}$ shown in table 14 of this report. The $1.35 \mathrm{Bgal} / \mathrm{d}$ decrease in the 2010 estimate for total withdrawals for mining was almost entirely accounted for in Oklahoma (86 percent) from revisions to estimates that resulted in reduced saline groundwater withdrawals. An increase of $1 \mathrm{Bgal} / \mathrm{d}$ for thermoelectric-power withdrawals

Table 14. Trends in estimated water use in the United States, 1950-2015.

[Data for 1980 and earlier are from Kenny and others (2009). Water-use data are in billion gallons per day (thousand million gallons per day) and are rounded to two significant figures for 1950-80, and to three significant figures for 1985-2015; percent change is calculated from unrounded numbers. Values may not sum to totals because of independent rounding. Geographic extent: 1950, 48 States, District of Columbia, and Hawaii; 1955 , 48 States and District of Columbia; 1960 and 1975-2015, 50 States, District of Columbia, Puerto Rico, and U.S. Virgin Islands; 1965-70, 50 States, District of Columbia, and Puerto Rico]

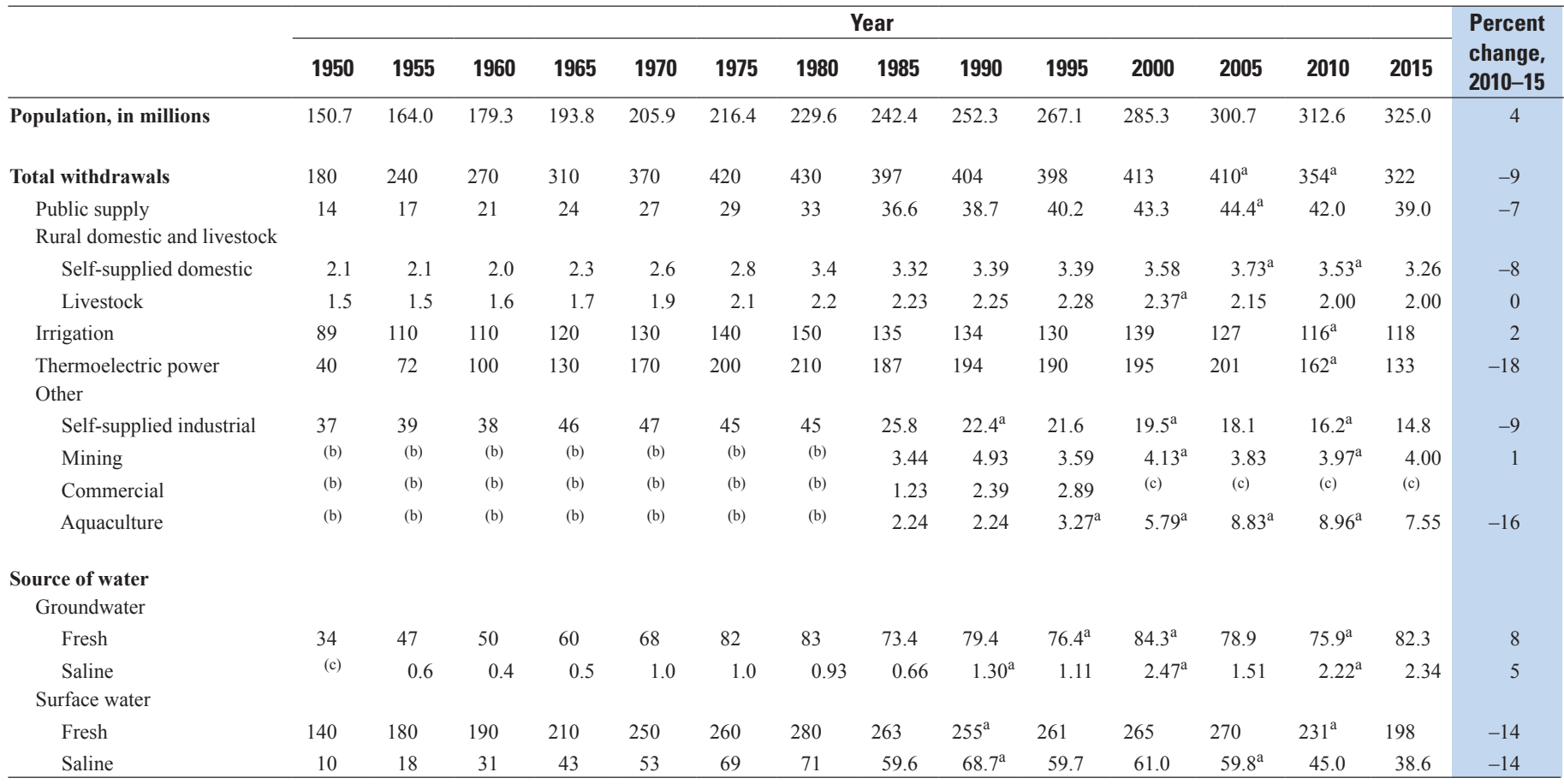

${ }^{a}$ Data revised from Maupin and others (2014) because of revisions to individual State data during interim years.

${ }^{\mathrm{b}}$ Included in self-supplied industrial.

${ }^{\mathrm{c} D a t a}$ not available. 


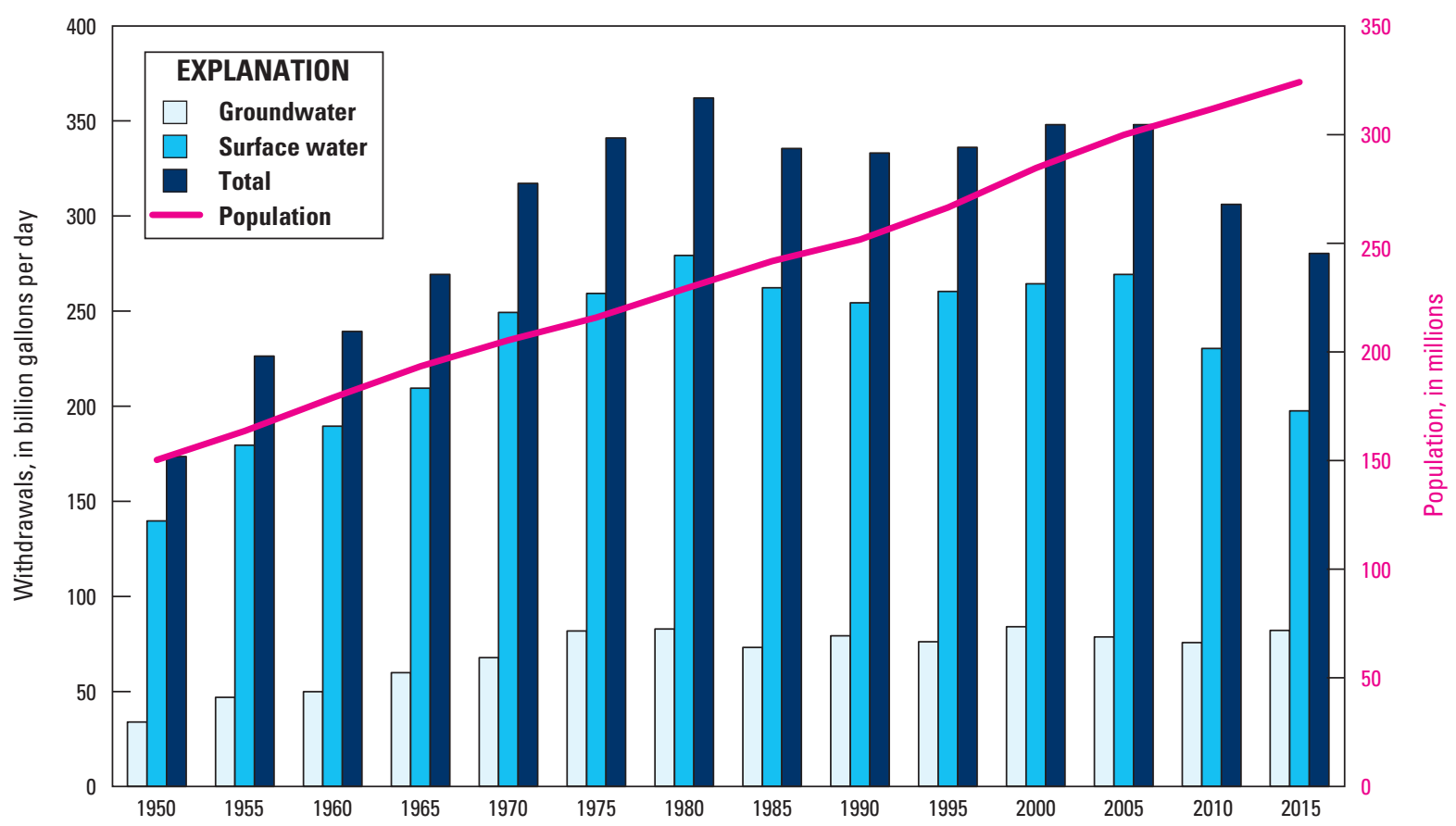

Figure 15. Trends in population and freshwater withdrawals by source, 1950-2015.

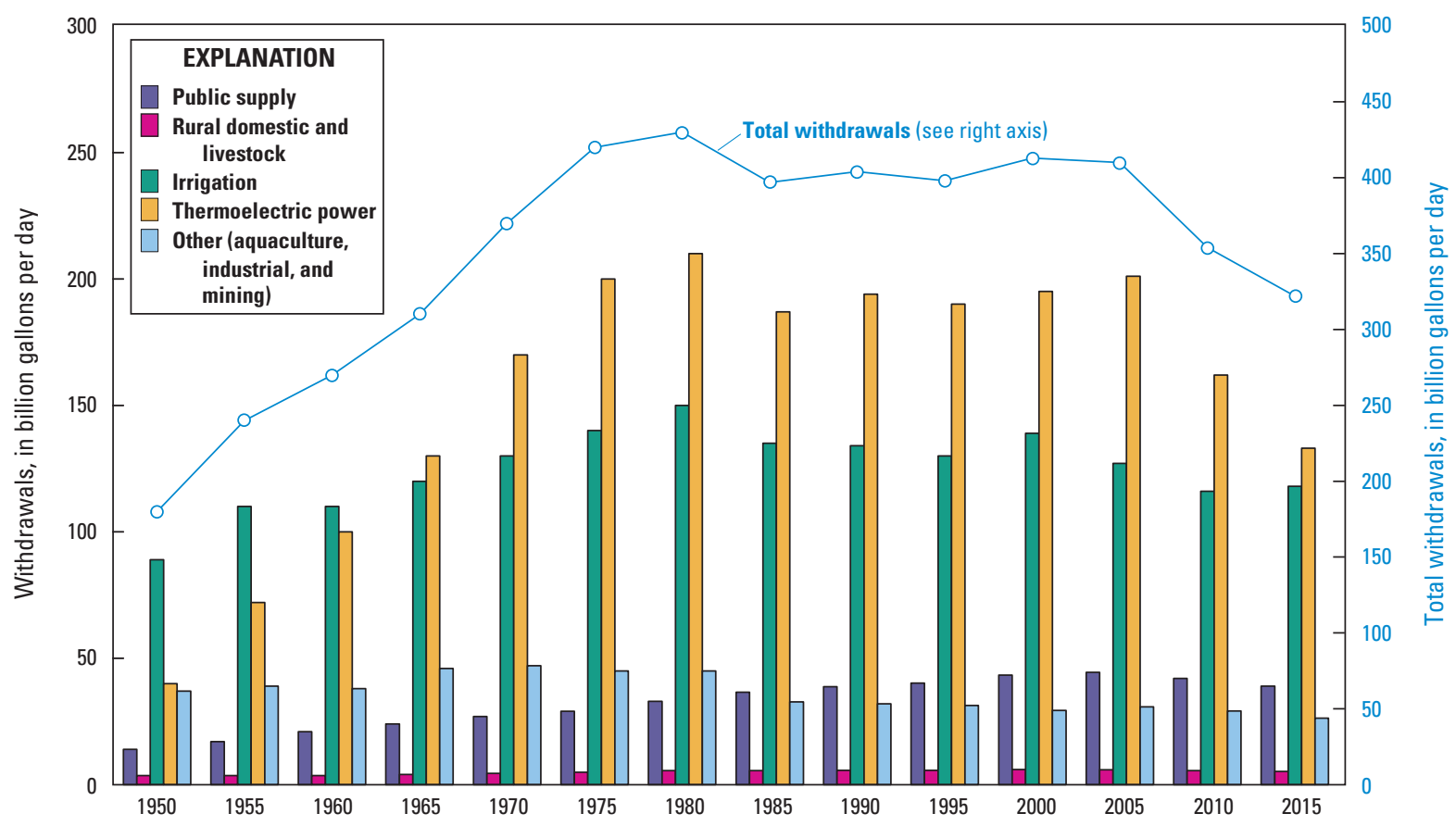

Figure 16. Trends in total water withdrawals by water-use category, 1950-2015. 
for 2010 published in Maupin and others (2014; table 14) versus the value in table 14 (this report) was entirely related to revisions in estimates for South Carolina. A small decrease $(0.46 \mathrm{Bgal} / \mathrm{d})$ for aquaculture withdrawals published in Maupin and others (2014; table 14) versus the value in table 14 (this report) was mostly accounted for by revisions to estimates, resulting in a decrease in Idaho and an increase in Colorado. The increase of $1 \mathrm{Bgal} / \mathrm{d}$ between published values for irrigation in Maupin and others (2014, table 14) versus the value in table 14 (this report) is caused by rounding; the actual difference amounted to only $0.182 \mathrm{Bgal} / \mathrm{d}$, mostly resulting from revisions to withdrawals in Montana. Table 14 notes additional total withdrawals by category for the United States from 1990 to 2015 that have changed since values were last published in Maupin and others (2014). These fluctuations in total withdrawals by category over time represent the level of flux that is inherent in maintaining large national datasets over time; datasets are constantly updated by USGS work or are based on new information from State, Federal, and local entities that are contributors to this national water-use compilation effort.

Comparisons between years stated hereafter are based on updated historical data and current 2015 data (table 14). Total withdrawals for all categories of use in 2015 were estimated to be $322 \mathrm{Bgal} / \mathrm{d}$, a level not reported since before 1970 and 9 percent less than in 2010, continuing a sharp but steady downward trend since 2005. The drop in total withdrawals in 2015 was primarily caused by significant decreases in withdrawals for thermoelectric power $(28.8 \mathrm{Bgal} / \mathrm{d})$, which accounted for 89 percent of the decrease in total withdrawals. The decrease in public-supply withdrawals accounted for another 9 percent of the decline in total withdrawals. Categories of use with larger withdrawals in 2015 than in 2010 were irrigation and mining. The increase in irrigation withdrawals was only about 2 percent greater than 2010 levels, partly resulting from updated 2010 data, and mining withdrawals were corrected downward for 2010.

States with the largest decreases in total withdrawals for all categories between 2010 and 2015 were California, Texas, Ohio, Illinois, Pennsylvania, and North Carolina, and cumulatively these six States accounted for more than two-thirds of the total decrease in withdrawals for all categories. States that showed the largest increases between 2010 and 2015 withdrawals were Wyoming, Arkansas, Montana, Nebraska and Idaho. Total groundwater withdrawals (mostly freshwater) increased about 6.52 Bgal/d between 2010 and 2015. States with the largest increases in groundwater withdrawals were California, Arkansas, Idaho, and Nebraska, mostly for irrigation uses. Total surface-water withdrawals decreased about $38.9 \mathrm{Bgal} / \mathrm{d}$, and the States that contributed to most of those decreased withdrawals were California, Texas, Ohio, Illinois, Pennsylvania, and North Carolina. These States, cumulatively, accounted for more than two-thirds of the total surface-water withdrawal decrease between 2010 and 2015, either through larger reductions in irrigation or thermoelectric-power withdrawals. California and Texas were experiencing recordsetting droughts during 2015, which also likely contributed to lower surface-water withdrawals because of the decrease in available water.

The total population in the United States increased 4 percent between 2010 and 2015, or 12.4 million people, almost exactly mirroring the increase between 2005 and 2010 (table 14; fig. 16). This continues the steady upward trend in total population growth exhibited since 1950. Texas, California, and Florida all had population increases ranging from about 1.5 million (Florida) to 2.3 million (Texas). These three States accounted for 46 percent of the total population increase in the Nation.

Public-supply withdrawals in 2015 were 7 percent less than in 2010 , decreasing from $42.0 \mathrm{Bgal} / \mathrm{d}$ to $39.0 \mathrm{Bgal} / \mathrm{d}$ and continuing the decline in public-supply withdrawals that was first observed in 2010 (table 14), which marked the first decline since being initially reported in 1950 (fig. 17A). Public-supply withdrawals increased between 2010 and 2015 in 17 States, with Florida showing the largest increase of about $0.12 \mathrm{Bgal} / \mathrm{d}$. States with the largest decreases in public-supply withdrawals were California and Texas (1.15 Bgal/d and $1.11 \mathrm{Bgal} / \mathrm{d}$, respectively), and they accounted for 76 percent of the national decrease of $2.99 \mathrm{Bgal} / \mathrm{d}$ between 2010 and 2015. The percentage of the population that is served from public-supply withdrawals has increased from 62 percent in 1950 to 87 percent in 2015. Texas, California, and Florida had the largest increases in the population that is served from public-supply withdrawals, accounting for 55 percent of the total national increase. Although California and Texas reported increased population served by public supply, withdrawals decreased. In 2015, California experienced a severe drought, and Governor Jerry Brown ordered a 25-percent statewide reduction in urban water use (which included water for landscaping) from water withdrawals in 2013 (Boxall and Xia, 2015). Selected water districts were ordered to reduce water usage by 36 percent, including water districts in the Sacramento region (Reese and Kasler, 2015). Reports from California water districts recorded a statewide reduction in water use of 27 and 31 percent in the summer months of June and July 2015, respectively, from the same months in 2013 (Boxall and Xia, 2015), exceeding the Governor's ordered limit. Although Texas was experiencing a drought in 2015 that had officially started in 2010 (National Oceanic and Atmospheric Administration, 2018), no mandatory water restrictions from the Governor were ordered in the State; however, municipal utilities in cities in Texas have been working with the populations they serve to promote water conservation. The San Antonio Water System that serves 1.6 million people, for example, reduced per capita water usage by 42 percent, simply by focusing on education, outreach, and regulations, through tools promoting water-smart landscaping, seminars on maintaining soils, and year-round time restrictions on the use of irrigation and sprinkler systems (U.S. Environmental Protection Agency, 2016). 

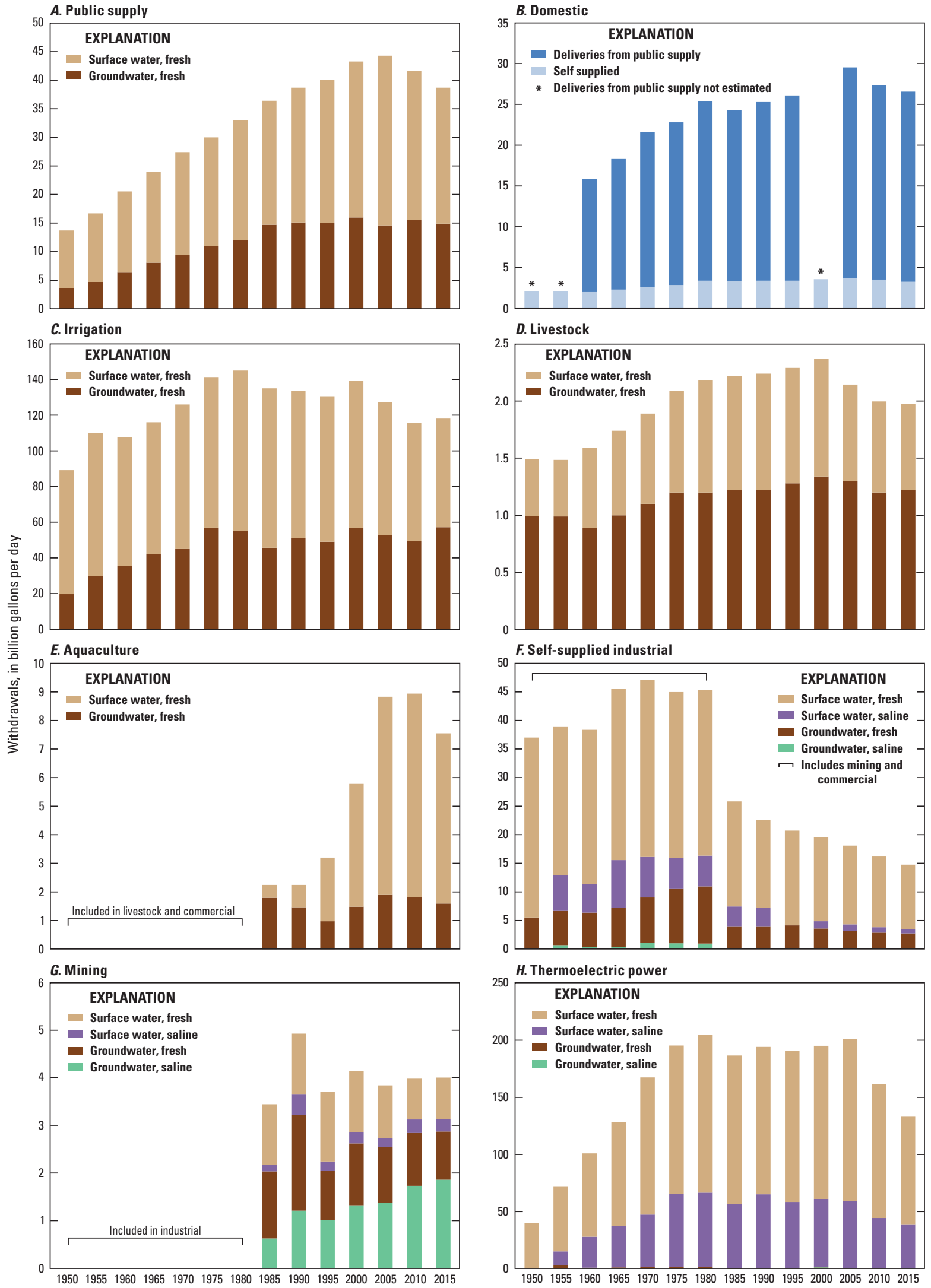

Figure 17. Trends in water withdrawals, 1950-2015. 
The self-supplied domestic withdrawals decreased by 8 percent from $3.53 \mathrm{Bgal} / \mathrm{d}$ in 2010 to $3.26 \mathrm{Bgal} / \mathrm{d}$ in 2015 . This represents a continued decline since 2005 and marks the lowest levels since prior to 1980 (table 14; fig. 17B). Between 1985 and 2015, the percentage of total population that was self-supplied has continuously declined, from about 18 percent to 13 percent.

The national average per capita use rate for self-supplied domestic withdrawals decreased from 81 GPCD in 2010 to 77 GPCD in 2015. This represents the rate of use for people with their own water supply. The rate of use for water that is delivered to people from public-supply systems (public-supply delivery GPCD) also decreased from 88 GPCD in 2010 to 82 GPCD in 2015.

Total domestic use is the combined water from publicsupply deliveries to domestic users and self-supplied domestic withdrawals. Nationally, these withdrawals decreased about $0.75 \mathrm{Bgal} / \mathrm{d}$ between 2010 and 2015, from $27.3 \mathrm{Bgal} / \mathrm{d}$ in 2010 to $26.6 \mathrm{Bgal} / \mathrm{d}$ in 2015 . Total domestic use in California alone decreased $0.68 \mathrm{Bgal} / \mathrm{d}$ between 2010 and 2015, accounting for 91 percent of the total national decrease and representing a 17-percent decrease in California's total domestic use between 2010 and 2015. In 2015, California endured one of the worst drought years on record (Khokha, 2014; Richtel, 2015; Stephens, 2015; California Department of Water Resources, 2016), with wide-scale water-use reductions and curtailments in effect statewide. Similarly, Texas was in a drought year in 2015 and reported about $0.05 \mathrm{Bgal} / \mathrm{d}$, or 2 percent, less total domestic use between 2010 and 2015.

Reductions from 2005 to 2015 in water withdrawals for public supply and self-supplied domestic can be attributed to Federal, State, and local policies that have allowed for water and energy efficiency improvements. One policy is the National Energy Policy Act of 1992 (Donnelly and Cooley, 2015; Osann, 2017). This act established efficiency standards for all toilets, faucets, and showerheads. Additional and amendment legislation improved the efficiency of washing machines, dishwashers, and other water using appliances (Donnelly and Cooley, 2015; Osann, 2017). Another program to encourage water efficiency is the EPA WaterSense launched in 2006; it is a voluntary program that uses an easily identifiable label on consumer products. Many of these products are the water and energy efficient fixtures and appliances mandated through the National Energy Act of 1992 (U.S. Environmental Protection Agency, 2018a); however, the WaterSense label also currently (2018) extends to irrigation and landscaping, buildings, and certification of irrigation professionals. WaterSense-labeled products are certified to use at least 20 percent less water and save energy.

Total irrigation withdrawals were somewhat similar between 2010 and 2015 with about a 2-percent increase from $116 \mathrm{Bgal} / \mathrm{d}$ to $118 \mathrm{Bgal} / \mathrm{d}$. Irrigation withdrawals for 2015 are at the fifth lowest level since 1950 and are 21 percent less than 1980, when withdrawals peaked at $150 \mathrm{Bgal} / \mathrm{d}$ (table 14; fig. 17C). However, 2015 marks the first 5-year period of increase in irrigation withdrawals since 2000, although it is possible that the increase is within the error of the estimated total withdrawal and that the withdrawals in 2015 are essentially the same as in 2010. California and Texas experienced the largest declines in irrigation withdrawals between 2010 and 2015, with a combined total reduction of $5.42 \mathrm{Bgal} / \mathrm{d}$. However, States with increased irrigation withdrawals between 2010 and 2015 include Wyoming, Arkansas, Montana, and Idaho. These States had a combined total increase of $9.59 \mathrm{Bgal} / \mathrm{d}$. Irrigation remains the secondlargest category of use after thermoelectric, but the difference between irrigation withdrawals and thermoelectric is narrowing. Irrigation withdrawals in 2015 accounted for 64 percent of total freshwater withdrawals, excluding thermoelectric, the same percentage as in 1950. Surface water continues to be the primary source of irrigation water in the United States, but the relative proportion appears to be changing. Between 1985 and 2010 , most of the irrigation water was supplied by surfacewater sources, ranging from 66 percent in 1985 to 57 percent in 2010. Surface water accounted for only 52 percent of the total irrigation withdrawals in 2015. Although reclaimed wastewater is a minor overall source of irrigation water in the Nation, accounting for less than 1 percent of the total irrigation water, its use increased from $0.47 \mathrm{Bgal} / \mathrm{d}$ in 2010 to about $0.67 \mathrm{Bgal} / \mathrm{d}$ in 2015 .

The use of more water-efficient irrigation systems continued to increase with 10 percent more irrigated acres using sprinkler systems in 2015 than in 2010. About 11 percent fewer irrigated acres were reported using surface (flood) irrigation systems in 2015 than in 2010. About 19 percent more irrigated acres were reported using microirrigation systems between 2010 and 2015. Total irrigated acres were about 2 percent more in 2015 than in 2010.

The compilation of irrigation water-use data conducted every 5 years since 1950 not only makes it possible to observe long-term changes in irrigation practices, including changing irrigation system types (for example, conversion of surface irrigation to sprinkler irrigation) and changes in total irrigated acres, but also allows for observations of shorter-term dynamic changes in irrigation practices and water use. For example, the 2015 dataset revealed the dynamic change in the Nation's largest user of water for irrigation, California. Estimated 2015 California irrigation withdrawals declined 4.07 Bgal/d (18 percent), and irrigated acres decreased by 10 percent in comparison to 2010, likely as a result of the intense drought conditions in 2015. Historically (1950-2010), surface water has been the primary source of irrigation water in California. However, groundwater was the primary source of irrigation water in California in 2015, likely as a result of limited available surface-water resources during the period of intense drought. In California in 2015, groundwater withdrawals for irrigation increased 60 percent from 2010, and surface-water withdrawals for irrigation decreased 64 percent.

Livestock initially was included with rural domestic but since 1960 has been estimated as a separate category and has consistently accounted for about 1-2 percent of total withdrawals, excluding thermoelectric. Total withdrawals 
for livestock were 2.00 Bgal/d in 2015, the same as in 2010 (table 14; fig. 17D). Livestock withdrawals in 2015 were 16 percent less than the peak year of 2000, when $2.37 \mathrm{Bgal} / \mathrm{d}$ was reported. This may, in part, be a reflection of the decreasing number of cattle and calves raised in the United States; the USDA NASS reported that the number of cattle and calves in the United States decreased 23 percent from 1,188,659 in 1997 to 913,246 in 2012 (U.S. Department of Agriculture, 2014a). Withdrawals for livestock are heavily influenced by numbers of cattle, especially milk cows, which generally use much more water than other livestock because of their size and the sanitation needs of milking facilities.

Aquaculture withdrawals were $7.55 \mathrm{Bgal} / \mathrm{d}$ in 2015 , or a 16 percent decrease from 2010 (8.95 Bgal/d). From 1985 to 2010 , aquaculture quadrupled from $2.24 \mathrm{Bgal} / \mathrm{d}$ in 1985 to $8.96 \mathrm{Bgal} / \mathrm{d}$ in 2010. Much of the increase occurred between 1995 and 2005 and is attributed to increased accounting of aquaculture withdrawals. The decrease in aquaculture withdrawals after 2010 (table 14; fig. 17E) marks the first period of decline since 1985, but the reason for the decline is unclear. The decrease could be a reflection of a sharp decrease in the reported number of aquaculture farms in the United States from 4,309 to 3,093 and in freshwater acreage used for aquaculture from 365,566 to 249,274 between 2005 and 2013 (U.S. Department of Agriculture, 2014b). The reported number of flow-through raceways, however, increased by 77 percent from 9,160 to 16,253 during the same period (U.S. Department of Agriculture, 2014b). However, the decrease could simply be due to differences in changes in methodologies that were used to estimate withdrawals for many of the States. The 2005 and 2015 water withdrawals for many States were estimated on the basis of data from the USDA NASS 2005 and 2013 censuses of aquaculture. The USDA NASS did not conduct a comparable census for 2010; therefore, the 2010 withdrawal estimates for many States were based on changes in the numbers of aquaculture operations from the USDA NASS 2002 and 2007 censuses of agriculture.

Self-supplied industrial withdrawals in the Nation can be directly compared from 1985 through 2015 . Since 1985, self-supplied industrial withdrawals have consistently declined and are about 43 percent less than 1985 (table 14; fig. 17F). Between 2010 and 2015, self-supplied industrial withdrawals decreased 1.4 Bgal/d. Texas $(-0.37 \mathrm{Bgal} / \mathrm{d})$, West Virginia (-0.34 Bgal/d), Pennsylvania ( $-0.22 \mathrm{Bgal} / \mathrm{d})$, Maryland ( $-0.15 \mathrm{Bgal} / \mathrm{d})$, Ohio (-0.14 Bgal/d), Arkansas ( $-0.12 \mathrm{Bgal} / \mathrm{d})$, and Michigan $(-0.10 \mathrm{Bgal} / \mathrm{d})$ had the largest decreases.

Delaware $(0.21 \mathrm{Bgal} / \mathrm{d})$ and Connecticut $(0.12 \mathrm{Bgal} / \mathrm{d})$ showed the largest increases from 2010 to 2015.

Declines in self-supplied industrial water withdrawals between 1985 and 2015 can likely be linked to a number of changes in factors in the United States economy, including technology, trade with foreign markets, operating costs, and a decline in manufacturing output (Bailey and Bosworth, 2014), as well as increases in the efficient use of water in industrial processes. One partial explanation for the decline in industry in the United States is a shift to a more service-based economy, where financial and health-care services have grown while the higher standard of living and higher labor costs in the United States have sent industries to countries with lowerpaid labor (Amadeo, 2017). A decline in manufacturing (and a loss of jobs in this sector) in the decade 2000 to 2009 (this would impact the 2005 and 2010 water-use data) is linked to a decline in manufacturing's share of gross domestic product; the products from the United States could not compete globally (Atkinson, 2013; Bailey and Bosworth, 2014). Finally, it has been suggested that higher taxes for manufactured goods in the United States, as well as decreased prices for overseas supplies better negotiated by other nations through trade agreements, make industrial products from the United States more expensive and less able to compete in the global market (Amadeo, 2017), resulting in less need for these manufactured goods.

In addition to changes in the United States economy, declines in self-supplied industrial withdrawals reflect greater efficiencies in industrial processes and an emphasis on water reuse and recycling within industrial facilities, both driven by environmental regulations and limited availability of freshwater resources in some areas. The EPA EnergyStar program, begun in 1992, provides information and suggestions on reducing water and energy use to industries (U.S. Environmental Protection Agency, 2018b).

Mining withdrawals were $4.00 \mathrm{Bgal} / \mathrm{d}$ in 2015, about a 1-percent increase from 2010 (3.97 Bgal/d). Prior to 1985, mining was included in other industrial withdrawals but since 1985 has represented from about 2 to 3 percent of total withdrawals, excluding thermoelectric. Trends in mining withdrawals fluctuated between 1985 and 2015, ranging from an increase of 43 percent between 1985 and 1990 to a 27-percent decrease between 1990 and 1995 (table 14; fig. $17 G$ ). Since 2000 , mining withdrawals have fluctuated only slightly, averaging about $4 \mathrm{Bgal} / \mathrm{d}$ over the period.

Thermoelectric-power withdrawals continued to account for the largest portion of total withdrawals for all categories of use, at $133 \mathrm{Bgal} / \mathrm{d}$, or 41 percent of the total withdrawals. However, total thermoelectric-power withdrawals in 2015 were about 18 percent less than in 2010 . On average, 15 gallons of water were used to produce $1 \mathrm{kWh}$ of electricity in 2015, compared to almost 19 gallons in 2010. The decline in withdrawals between 2010 and 2015 continues the trend of declining withdrawals for thermoelectric power; withdrawals in 2010 were about 20 percent less than in 2005 (table 14; fig. 17H). Total withdrawals for thermoelectric power in 1985 were 11 percent less than in 1980, and fluctuations in total withdrawals during the 5-year intervals between 1985 and 2005 were never more than 5 percent. States with the largest increases in thermoelectric-power withdrawals between 2010 and 2015 were Nebraska (1.12 Bgal/d), Florida (0.67 Bgal/d), and North Dakota (0.15 Bgal/d). Many States (California, Ohio, Illinois, Pennsylvania, Alabama, Indiana, Massachusetts, Kentucky, North Carolina, Tennessee, and Georgia) had large decreases in withdrawals, ranging from $3.73 \mathrm{Bgal} / \mathrm{d}$ (California) to $1.17 \mathrm{Bgal} / \mathrm{d}$ (Georgia). 
Several factors may have contributed to the 18 percent decline in total thermoelectric withdrawals between 2010 and 2015 (table 14). Since the 1970s, an increasing number of powerplants were built with or converted to recirculating cooling systems or dry cooling systems, which withdraw less water than powerplants with once-through cooling systems. Since 2010, 77 percent of the new cooling systems are recirculating cooling systems, and 18 percent are dry cooling systems (U.S. Department of Energy, 2016a). The increased use of natural gas to power energy- and water-efficient combined-cycle plants, almost all of which have recirculating or dry-cooling towers, has contributed to the decline in withdrawals (U.S. Department of Energy, 2016a, c). The decreased use of coal has also helped to reduce withdrawals because many coal-fired powerplants with once-through cooling systems have been retired. Of the 49 plants with once-through cooling systems that were generating electricity in 2010 and have since been decommissioned, 31 of them were conventional coal-fired powerplants (U.S. Department of Energy, 2016a). Withdrawals have declined in some States as a result of the implementation of new rules designed to minimize adverse effects to aquatic life from heated discharges and from entrainment/impingement at pump intakes at powerplants with once-through cooling systems (California Environmental Protection Agency, 2010).

\section{References Cited}

Amadeo, K., 2017, U.S. manufacturing - What it is, statistics, and outlook: The Balance, December 6, 2017, accessed January 17, 2018, at https://www.thebalance.com/u-smanufacturing-what-it-is-statistics-and-outlook-3305575.

Atkinson, R., 2013, Why the 2000s were a lost decade for American manufacturing: Industry Week, March 14, 2013, accessed January 18, 2018, at http://www.industryweek. com/the-2000s.

Bailey, M.N., and Bosworth, B.P., 2014, U.S. manufacturing-Understanding its past and its potential future: Journal of Economic Perspectives, v. 28, no. 1, p. 3-26.

Boxall, B., and Xia, R., 2015, Big month for conservationCalifornians cut water use by $31 \%$ in July: Los Angeles Times, August 27, 2015, accessed February 22, 2018, at http://www.latimes.com/local/lanow/la-me-ln-july-urbanwater-savings-20150827-story.html.

Bradley, M.W., comp., 2017, Guidelines for preparation of State water-use estimates for 2015: U.S. Geological Survey Open-File Report 2017-1029, 54 p., accessed March 2018, at https://doi.org/10.3133/ofr20171029.
California Department of Water Resources, 2016, Drought information news archive: California Department of Water Resources web page, accessed March 19, 2018, at https://drought.ca.gov/archives.html.

California Environmental Protection Agency, 2010, Policy on the use of coastal and estuarine waters for power plant cooling: State Water Resources Control Board web page, accessed February 2018, at https:/www.waterboards. ca.gov/water_issues/programs/ocean/cwa316/policy.shtml.

Clark County Indiana Community Portal [2018], Fifth-largest steel producer in the world coming to the Port of Indiana: Clark County Indiana Community Portal web page, accessed February 28, 2018, at https://www.co.clark.in.us/ index.php/business/clark-county-indiana-business-news/ 95-fifth-largest-steel-producer-in-the-world-coming-to-theport-of-indiana.

Diehl, T.H., and Harris, M.A., 2014, Withdrawals and consumption of water by thermoelectric power plants in the United States, 2010: U.S. Geological Survey Scientific Investigations Report 2014-5184, 28 p., accessed March 2018, at https://doi.org/10.3133/sir20145184.

Diehl, T.H., Harris, M.A., Murphy, J.C., Hutson, S.S., and Ladd, D.E., 2013, Methods for estimating water consumption for thermoelectric power plants in the United States: U.S. Geological Survey Scientific Investigations Report 2013-5188, 78 p., accessed March 2018, at https://doi.org/10.3133/sir20135188.

Dieter, C.A., Linsey, K.S., Caldwell, R.R., Harris, M.A., Ivahnenko, T.I., Lovelace, J.K., Maupin, M.A., and Barber, N.L., 2017, Estimated use of water in the United States county-level data for 2015 (version 1.0, September 2017): U.S. Geological Survey data release, https://doi.org/10.5066/F7TB15V5.

Dieter, C.A., Linsey, K.S., Caldwell, R.R., Harris, M.A., Ivahnenko, T.I., Lovelace, J.K., Maupin, M.A., and Barber, N.L., 2018, Estimated use of water in the United States county-level data for 2015 (version 2.0, June 2018): U.S. Geological Survey data release, https://doi.org/10.5066/F7TB15V5.

Dieter, C.A., and Maupin, M.A., 2017, Public supply and domestic water use in the United States, 2015: U.S. Geological Survey Open-File Report 2017-1131, 6 p., https://doi.org/10.3133/ofr20171131.

Donnelly, K., and Cooley, H., 2015, Water use trends in the United States: Pacific Institute, 12 p., accessed January 25, 2018, at https:/www.pacinst.org/wp-content/ uploads/2015/04/Water-Use-Trends-Report.pdf. 
Downie, R., 2016, Texas' economy-The 9 industries driving GDP growth: Investopedia, January 13, 2016, accessed January 16, 2018, at https://www.investopedia. com/articles/investing/011316/texas-economy-9-industriesdriving-gdp-growth.asp.

FracFocus, 2016, FracFocus chemical disclosure registry: FracFocus database, accessed October 28, 2016, at http://fracfocus.org/data-download.

Groenfeldt, T., 2015, Top 5 industries in Indiana-Which parts of the economy are the strongest?: Newsmax, April 5, 2015, accessed January 11, 2018, at https://www.newsmax. com/fastfeatures/industries-indiana-economy/2015/04/05/ id/636527/.

Harris, M.A., and Diehl, T.H., 2017, A comparison of three federal datasets for thermoelectric water withdrawals in the United States for 2010: Journal of the American Water Resources Association, v. 53, no. 5, p. 1062-1080, accessed March 2018, at https://doi.org/10.1111/1752-1688.12551.

Hutson, S.S., Barber, N.L., Kenny, J.F., Linsey, K.S., Lumia, D.S., and Maupin, M.A., 2004, Estimated use of water in the United States in 2000: U.S. Geological Survey Circular 1268, 46 p., https://pubs.usgs.gov/circ/2004/circ1268/.

Indiana Department of Natural Resources, 2016, Division of water, rights and use, significant water withdrawal facility database: Indiana Department of Natural Resources, accessed September 15, 2016, at http://www.in.gov/dnr/ water/4841.htm.

Jones, S., 2015, Top 5 industries in Louisiana-Which parts of the economy are the strongest?: Newsmax, April 8, 2015, accessed January 11, 2018, at https://www.newsmax.com/ fastfeatures/industries-louisiana-economy/2015/04/08/ $\mathrm{id} / 637289 /$.

Kenny, J.F., Barber, N.L., Hutson, S.S., Linsey, K.S., Lovelace, J.K., and Maupin, M.A., 2009, Estimated use of water in the United States in 2005: U.S. Geological Survey Circular 1344, 52 p., accessed March 2018, at https://pubs. usgs.gov/circ/1344/.

Khokha, S., 2014, As their wells run dry, California residents blame thirsty farms: National Public Radio, October 19, 2014, accessed October 5, 2017, at http://www.npr. org/2014/10/19/357273445/as-their-wells-run-drycalifornia-residents-blame-thirsty-farms.

Lovelace, J.K., 2009a, Method for estimating water withdrawals for livestock in the United States, 2005: U.S. Geological Survey Scientific Investigations Report 2009-5041, 7 p., accessed March 2018, at https://pubs.usgs.gov/ sir/2009/5041/pdf/sir2009-5041.pdf.
Lovelace, J.K, 2009b, Methods for estimating water withdrawals for aquaculture in the United States, 2005: U.S. Geological Survey Scientific Investigations Report 2009-5042, 13 p., accessed March 2018, at https://pubs.usgs.gov/ sir/2009/5042/pdf/sir2009-5042.pdf.

Lovelace, J.K., 2009c, Methods for estimating water withdrawals for mining in the United States, 2005: U.S. Geological Survey Scientific Investigations Report 2009-5053, 7 p., accessed March 2018, at https://pubs.usgs.gov/ sir/2009/5053/pdf/sir2009-5053.pdf.

MacKichan, K.A., 1951, Estimated use of water in the United States, 1950: U.S. Geological Survey Circular 115, 13 p.

MacKichan, K.A., 1957, Estimated use of water in the United States, 1955: U.S. Geological Survey Circular 398, 18 p.

MacKichan, K.A., and Kammerer, J.C., 1961, Estimated use of water in the United States, 1960: U.S. Geological Survey Circular 456, $26 \mathrm{p}$.

Maupin, M.A., Kenny, J.F., Hutson, S.S., Lovelace, J.K., Barber, N.L., and Linsey, K.S., 2014, Estimated use of water in the United States in 2010: U.S. Geological Survey Circular 1405, 56 p., accessed October 3, 2017, at https:// doi.org/10.3133/cir1405.

Murray, C.R., 1968, Estimated use of water in the United States, 1965: U.S. Geological Survey Circular 556, 53 p.

Murray, C.R., and Reeves, E.B., 1972, Estimated use of water in the United States, 1970: U.S. Geological Survey Circular $676,37 \mathrm{p}$.

Murray, C.R., and Reeves, E.B., 1977, Estimated use of water in the United States in 1975: U.S. Geological Survey Circu$\operatorname{lar} 765,39 \mathrm{p}$.

National Oceanic and Atmospheric Administration, 2016a, State of the climate; National climate report for annual 2015: National Centers for Environmental Information web page, accessed January 24, 2018, at https://www.ncdc.noaa. gov/sotc/national/201513.

National Oceanic and Atmospheric Administration, 2016b, State of the climate; Drought for annual 2015: National Centers for Environmental Information web page, accessed February 4, 2018, at https://www.ncdc.noaa.gov/sotc/ drought/201513.

National Oceanic and Atmospheric Administration [2018], From a dry Texas autumn to exceptional drought and back: National Integrated Drought Information System web page, accessed February 22, 2018, at https://www.drought.gov/ drought/dry-texas-autumn-exceptional-drought-and-back. 
Osann, E., 2017, 25 years of water efficiency across the US: National Resources Defense Council, October 25, 2017, accessed March 2018, at https://www.nrdc.org/ experts/ed-osann/celebrating-25-years-water-efficiency.

Reese, P., and Kasler, D., 2015, California water regulators approve massive cutback orders: The Sacramento Bee, May 5, 2015, accessed February 22, 2018, at http:/www. sacbee.com/news/state/california/water-and-drought/ article20277303.html.

Richtel, M., 2015, California farmers dig deeper for water, sipping their neighbors dry: The New York Times, June 5, 2105, accessed October 5, 2017, at https:/www.nytimes. com/2015/06/07/business/energy-environment/californiafarmers-dig-deeper-for-water-sipping-their-neighbors-dry. html?mcubz=3.

Senay, G.B., Bohms, S., Singh, R.K., Gowda, P.H., Velpuri, N.M., Alemu, H., and Verdin, J.P., 2013, Operational evapotranspiration mapping using remote sensing and weather datasets-A new parameterization for the SSEB approach: Journal of the American Water Resources Association, v. 49, no. 3, p. 577-591, accessed March 2018, at https://doi.org/ 10.1111/jawr.12057.

Solley, W.B., Chase, E.B., and Mann, W.B., IV, 1983, Estimated use of water in the United States in 1980: U.S. Geological Survey Circular 1001, 56 p. [Also available at https://pubs.er.usgs.gov/publication/cir1001.]

Solley, W.B., Merk, C.F., and Pierce, R.R., 1988, Estimated use of water in the United States in 1985: U.S. Geological Survey Circular 1004, 82 p. [Also available at https://pubs. er.usgs.gov/publication/cir1004.]

Solley, W.B., Pierce, R.R., and Perlman, H.A., 1993, Estimated use of water in the United States in 1990: U.S. Geological Survey Circular 1081, 76 p. [Also available at https://pubs.er.usgs.gov/publication/cir1081.]

Solley, W.B., Pierce, R.R., and Perlman, H.A., 1998, Estimated use of water in the United States in 1995: U.S. Geological Survey Circular 1200, 71 p. [Also available at https://pubs.er.usgs.gov/publication/cir1200.]

Stephens, M., 2015, Water, 2015, California; The no-good, very bad year - now, 'pray for rain': Los Angeles Times, September 29, 2015, accessed October 5, 2017, at http:// www.latimes.com/local/lanow/la-me-ln-water-year20150929-story.html.

U.S. Census Bureau, 2016, Population, population change and estimated components of population changeApril 1, 2010 to July 1, 2015: U.S. Census Bureau, accessed March 28, 2016, at http://www.census.gov/popest/data/ counties/totals/2015/index.html.
U.S. Census Bureau, 2017, Annual population estimates, estimated components of resident population change, and rates of the components of resident population change for the United States and Puerto Rico-April 1, 2010 to July 1, 2017: United States Census Bureau, Population Division data release, accessed January 25, 2018, at https://www2. census.gov/programs-surveys/popest/datasets/2010-2017/ national/totals/nst-est2017-alldata.csv.

U.S. Department of Agriculture, 2014a, 2012 Census of agriculture - United States summary and state data: National Agricultural Statistics Service, Geographic Area Series, v. 1, part 51, AC-12-A-51, 695 p., accessed January 26, 2018, at https://www.agcensus.usda.gov/ Publications/2012/Full_Report/Volume_1,_Chapter_1_US/ usv1.pdf.

U.S. Department of Agriculture, 2014b, 2012 Census of agriculture - Census of aquaculture (2013): National Agricultural Statistics Service, Special Studies, v. 3, part 2, AC-12-SS-2, 98 p., accessed January 25, 2018, at https:// www.agcensus.usda.gov/Publications/2012/Online_ Resources/Aquaculture/Aqua.pdf.

U.S. Department of Energy, 2016a, 2015 Form EIA-860 database: U.S. Energy Information Administration, Annual Electric Generator Report, accessed January 2018, at http:// www.eia.gov/electricity/data/eia860/index.html.

U.S. Department of Energy, 2016b, 2015 Form EIA-923 database: U.S. Energy Information Administration, Power Plant Operations Report, accessed January 2018, at http://www. eia.gov/electricity/data/eia923/.

U.S. Department of Energy, 2016c, Annual energy outlook 2016 with projections to 2040: U.S. Energy Information Administration, Office of Energy Analysis, DOE/EIA0383(2016), $256 \mathrm{p}$.

U.S. Environmental Protection Agency, 2016, Saving water in Texas: U.S. Environmental Protection Agency EPA832-F-16-005, accessed March 2018, at https:/www.epa. gov/sites/production/files/2017-02/documents/ws-ourwatertexas-state-fact-sheet.pdf.

U.S. Environmental Protection Agency, 2018a, Water and energy efficiency by sectors: U.S. Environmental Protection Agency web page, accessed January 23, 2018, at https:// www3.epa.gov/region9/waterinfrastructure/industry.html.

U.S. Environmental Protection Agency, 2018b, WaterSense: U.S. Environmental Protection Agency web page, accessed January 25, 2018, at https://www.epa.gov/watersense. 


\section{Glossary}

The following terms are referenced in the text or are part of the water-use Circular series.

animal-specialties water use Water use associated with the production of fish in captivity, except for fish hatcheries, and the raising of horses and such furbearing animals as rabbits and pets. Animal-specialties water-use estimates were included in the 1990 and 1995 water-use Circulars but were combined with the livestock categories or aquaculture categories beginning in 2000. See also aquaculture water use, fish-farm water use, livestock water use, and rural water use.

aquaculture water use Water use associated with the farming of organisms that live in water (such as finfish and shellfish) and offstream water use associated with fish hatcheries. See also fish-farm water use, fish-hatchery water use, animal-specialties water use, and livestock water use.

closed-loop cooling system See recirculating cooling system.

commercial water use Water for motels, hotels, restaurants, office buildings, other commercial facilities, military and nonmilitary institutions, and (for 1990 and 1995) offstream fish hatcheries. Water may be obtained from a public-supply system or may be self-supplied. Commercial water-use estimates were included in some previous water-use Circulars but were omitted beginning in 2000. See also fish-hatchery water use, public-supply water use, public-supply deliveries, and self-supplied water use.

consumptive use The part of water withdrawn that is evaporated, transpired, incorporated into products or crops, consumed by humans or livestock, or otherwise removed from the immediate water environment. Also referred to as water consumed.

conveyance loss Water that is lost in transit from a pipe, canal, conduit, or ditch by leakage or evaporation. Generally, the water is not available for further use; however, leakage from an irrigation ditch, for example, may percolate to a groundwater source and be available for further use. Conveyance-loss estimates were included in some previous water-use Circulars but were omitted beginning in 2000. See also irrigation water use.

cooling system An equipment system that provides water for cooling purposes, such as to condensers at powerplants or at factories. May include water intakes, outlets, cooling towers, ponds, canals, pumps, and pipes. See also cooling-system type, industrial water use, and thermoelectric-power water use. cooling-system type Defined as either once-through or recirculating cooling system. See also industrial water use, once-through cooling system, recirculating cooling system, and thermoelectric-power water use.

domestic water use Water used for indoor household purposes such as drinking, food preparation, bathing, washing clothes and dishes, flushing toilets, and outdoor purposes such as watering lawns and gardens. Domestic water use includes water provided to households by a public water supply (domestic deliveries from public suppliers) and self-supplied water. See also public-supply deliveries, public-supply water use, rural water use, and self-supplied water use.

fish-farm water use Water used for the production of finfish and shellfish under controlled feeding, sanitation, and harvesting procedures for commercial purposes. Water use by fish farms is classified in the aquaculture category. See also animal-specialties water use, aquaculture water use, and fish-hatchery water use.

fish-hatchery water use Water used for raising fish for later release and in association with the operation of fish hatcheries or fishing preserves. Fish-hatchery water use has been included in the aquaculture category since 2000. See also aquaculture water use, commercial water use, and fish-farm water use.

freshwater Water that contains less than 1,000 milligrams per liter $(\mathrm{mg} / \mathrm{L})$ of dissolved solids. Generally, water with more than $500 \mathrm{mg} / \mathrm{L}$ of dissolved solids is undesirable for drinking and many industrial uses. See also saline water.

industrial water use Water used for fabrication, processing, washing, and cooling. Includes industries such as chemical and allied products, food, mining, paper and allied products, petroleum refining, and steel Term used in previous water-use Circulars to describe the combined public-supply deliveries to industrial users and self-supplied industrial withdrawals. Since 2000 , industrial water use refers only to self-supplied industrial withdrawals. See also mining water use, public-supply deliveries, public-supply water use, and self-supplied water use. 
instream use Water that is used, but not withdrawn, from a surface-water source for such purposes as hydroelectric-power generation, navigation, waterquality improvement, fish propagation, and recreation. Instream water-use estimates for hydroelectric power were included in some previous water-use Circulars but were omitted since 2000. See also offstream use.

irrigation district A cooperative, self-governing public corporation set up as a subdivision of the State government, with definite geographic boundaries, organized, and having taxing power to obtain and distribute water for irrigation of lands within the district. Created under the authority of a State legislature with the consent of a designated fraction of the landowners or citizens. See also irrigation water use.

irrigation water use Water that is applied by an irrigation system to assist crop and pasture growth, or to maintain vegetation on recreational lands such as parks and golf courses. Irrigation includes water that is applied for pre-irrigation, frost protection, chemical application, weed control, field preparation, crop cooling, harvesting, dust suppression, leaching of salts from the root zone, and conveyance losses. Irrigation water use includes self-supplied water and reclaimed wastewater. See also conveyance loss, microirrigation system, sprinkler irrigation system, and surface irrigation system.

livestock water use Water used for livestock watering, feedlots, dairy operations, and other on-farm needs. Types of livestock include dairy cows and heifers, beef cattle and calves, sheep and lambs, goats, hogs and pigs, horses, and poultry. See also animal-specialties water use, aquaculture water use, and rural water use.

microirrigation system An irrigation system that wets only a discrete portion of the soil surface in the vicinity of the plant by means of applicators (such as orifices, emitters, porous tubing, or perforated pipe) and operated under low pressure. The applicators may be placed on or below the surface of the ground or suspended from supports. See also irrigation water use, sprinkler irrigation system, and surface irrigation system.

mining water use Water used for the extraction of naturally occurring minerals including solids (such as coal, sand, gravel, and other ores), liquids (such as crude petroleum), and gases (such as natural gas). Also includes uses associated with quarrying, milling of mined materials, injection of water for secondary oil recovery or for unconventional oil and gas recovery (such as hydraulic fracturing), and other operations associated with mining activity. Does not include water associated with dewatering of the aquifer that is not put to beneficial use. Also does not include water used in processing, such as smelting, refining petroleum, or slurry pipeline operations. These processing uses are included in industrial water use. See also industrial water use and self-supplied water use. offstream use Water withdrawn or diverted from a groundwater or surface-water source for aquaculture, commercial, self-supplied domestic, industrial, irrigation, livestock, mining, public supply, thermoelectric power, and other uses. See also entries for each of these categories of use, and instream use.

once-through cooling system Also known as open-loop cooling system. Cooling system in which the water is withdrawn from a source, circulated through the heat exchangers, and then returned to a body of water at a higher temperature. See also cooling system, coolingsystem type, and thermoelectric-power water use.

public-supply deliveries Amount of water delivered from a public supplier to users for domestic, commercial, industrial, thermoelectric-power, or public-use purposes. Estimates of deliveries for each purpose were provided for 1995 and earlier years, but not for 2000. For 2005-2015, only domestic deliveries were estimated nationally. See also commercial water use, domestic water use, industrial water use, public-supply water use, public water use, and thermoelectric-power use.

public-supply water use Water withdrawn by public and private water suppliers that furnish water to at least 25 people or have a minimum of 15 connections. Public suppliers provide water for a variety of uses, such as domestic, commercial, industrial, thermoelectric-power, and public water use. See also commercial water use, domestic water use, industrial water use, public-supply deliveries, public water use, and thermoelectric-power water use.

public water use Water supplied from a public supplier and used for such purposes as firefighting, street washing, flushing of water lines, and maintaining municipal parks and swimming pools. Generally, public-use water is not billed by the public supplier. See also public-supply deliveries and public-supply water use.

recirculating cooling system Also known as closed-loop cooling system or recirculation cooling system. Water is withdrawn from a source, circulated through heat exchangers, cooled, and then re-used in the same process. Recirculating cooling systems may use induced draft cooling towers, forced draft cooling towers, cooling ponds, or canals. See also cooling system, cooling-system type, and thermoelectric-power water use.

reclaimed wastewater Wastewater-treatment plant effluent that has been diverted for beneficial uses such as irrigation, industrial use, or thermoelectric-power cooling instead of being released to a natural waterway or aquifer. See also water use. 
return flow Water that reaches a groundwater or surface-water source after release from the point of use and thus becomes available for further use. See also water use.

rural water use Water used in suburban or farm areas for domestic and livestock needs. The water generally is self-supplied, and includes domestic use, drinking water for livestock, and other uses such as dairy sanitation, cleaning, and waste disposal. Term used in 1950 and 1955 water-use Circulars. See also animal-specialties water use, domestic water use, livestock water use, and self-supplied water use.

saline water Water that contains $1,000 \mathrm{mg} / \mathrm{L}$ or more of dissolved solids. See also freshwater.

self-supplied water use Water withdrawn from a groundwater or surface-water source by a user rather than being obtained from a public-supply source.

sprinkler irrigation system An irrigation system in which water is applied by means of perforated pipes or nozzles operated under pressure so as to form a spray pattern. See also irrigation water use, microirrigation system, and surface irrigation system.

surface irrigation system Irrigation by means of flood, furrow, or gravity methods. Flood irrigation is the application of irrigation water in which the entire soil surface is covered by ponded water. Furrow is a partial surface-flooding method of irrigation normally used with clean-tilled crops in which water is applied in furrows or rows of sufficient capacity to contain the design irrigation stream. Gravity is an irrigation method in which water is not pumped, but flows in ditches or pipes and is distributed by gravity. See also irrigation water use, microirrigation system, and sprinkler irrigation system. thermoelectric-power water use Water used in the process of generating electricity with steam-driven turbine generators. Thermoelectric-power water use includes water provided by a public water supply (deliveries from public suppliers), self-supplied water, and reclaimed wastewater. See also cooling system, cooling-system type, public-supply water use, reclaimed wastewater, and self-supplied water use.

wastewater-treatment return flow Term used in previous water-use Circulars to describe water returned to the hydrologic system by wastewater-treatment facilities. See also water use.

water use In a restrictive sense, the term refers to water that is withdrawn for a specific purpose, such as for public supply, domestic use, irrigation, thermoelectric-power cooling, or industrial processing. In some previous water-use Circulars, water use for the domestic, commercial, industrial, and thermoelectric categories included both self-supplied withdrawals and deliveries from public supply, and some categories included reclaimed wastewater use. More broadly, water use pertains to the interaction of humans with and influence on the hydrologic cycle, and includes elements such as water withdrawal, delivery, consumptive use, wastewater release, reclaimed wastewater, return flow, and instream use. See also offstream use and instream use.

water withdrawal Water removed from a groundwater or surface-water source for use. See also offstream use and self-supplied water use.

watt-hour (Wh) An electrical energy unit of measure equal to 1 watt of power supplied to, or taken from, an electric circuit steadily for 1 hour. 


\section{Contributing Agencies and Organizations}

The following State, regional, and local organizations provided assistance and data as part of the water-use compilation. In addition, State, regional, and national offices of the U.S. Army Corps of Engineers, U.S. Bureau of Reclamation, U.S. Census Bureau, U.S. Department of Energy, U.S. Environmental Protection Agency, U.S. Fish and Wildlife Service, National Agricultural Statistics Service, National Oceanic and Atmospheric Administration, National Weather Service, Natural Resources Conservation Service, and other Federal agencies provided assistance and data for various States.

\section{Alabama}

Alabama Department of Economic and Community

Development, Office of Water Resources

\section{Alaska}

Alaska Department of Commerce, Community and Economic Development, Community and Regional Affairs

Alaska Department of Environmental Conservation, Division of Water

Alaska Department of Fish and Game

Alaska Department of Natural Resources, Division of Mining, Land and Water

\section{Arizona}

Arizona Corporation Commission

Arizona Department of Environmental Quality

Arizona Department of Water Resources

Arizona Public Service

Buckeye Water Conservation and Drainage District

Central Arizona Project

Gila Water Commissioner

Paloma Irrigation and Drainage District

Salt River Project

Wellton-Mohawk Irrigation and Drainage District

\section{Arkansas}

Arkansas Association of Conservation Districts Arkansas Department of Health, Engineering Division Arkansas Natural Resources Commission

\section{California}

California Department of Conservation, Division of Land Resource Protection

California Department of Corrections and Rehabilitation

California Department of Water Resources

California Environmental Protection Agency, State Water Resources Control Board

Golf Course Superintendents Association of America

\section{Colorado}

Colorado Division of Reclamation Mining and Safety

Colorado Division of Water Resources

Colorado Oil and Gas Conservation Commission

Colorado Water Conservation Board

Rocky Mountain Golf Course Superintendents Association

\section{Connecticut}

State of Connecticut Department of Environmental Protection

\section{Delaware}

Delaware Department of Natural Resources and Environmental Control

Delaware State University

University of Delaware

\section{District of Columbia}

DC Water

\section{Florida}

Florida Department of Agriculture and Consumer Services Northwest Florida Water Management District

South Florida Water Management District Southwest Florida Water Management District St. Johns River Water Management District

Suwannee River Water Management District

\section{Georgia}

Georgia Environmental Protection Division, Watershed Protection Branch

Georgia Power Company

Georgia Water Planning and Policy Center 


\section{Hawaii}

Hawaii Department of Water Supply

Hawaiian Electric Company

Honolulu Board of Water Supply

Kauai Department of Water

Maui Department of Water Supply

\section{Idaho}

Idaho Chapter Golf Course Superintendents Association of America

Idaho Department of Commerce

Idaho Department of Environmental Quality

Idaho Department of Fish and Game

Idaho Department of Water Resources

\section{Illinois}

Illinois State Water Survey-Illinois Water Inventory Program Imperial Valley Water Authority

\section{Indiana}

Indiana Department of Natural Resources, Division of Water, Water Rights, and Use Section

\section{lowa}

Iowa Department of Natural Resources-Water Allocation and Use Program

\section{Kansas}

Kansas Department of Agriculture-Division of Water Resources

Kansas Water Office

\section{Kentucky}

Kentucky Energy and Environment Cabinet, Department of Environmental Protection, Division of Water

\section{Louisiana}

Capitol Area Ground Water Conservation Committee Louisiana Cooperative Extension Service

Louisiana Department of Health and Hospitals Louisiana Department of Natural Resources Louisiana Department of Transportation and Development Louisiana State University Agricultural Center

\section{Maine}

Maine Department of Agriculture, Conservation and Forestry Maine Department of Environmental Protection Maine Department of Health and Human Services Maine Geological Survey

Maine Public Utilities Commission

\section{Maryland}

Maryland Department of the Environment

\section{Massachusetts}

Massachusetts Department of Environmental Protection

\section{Michigan}

Michigan Department of Environmental Quality, Water Use Program

Michigan Department of Agriculture and Rural Development

\section{Minnesota}

Minnesota Department of Natural Resources

\section{Mississippi}

Mississippi Department of Environmental Quality, Office of Land and Water Resources Mississippi State Department of Health Mississippi State Oil and Gas Board Mississippi State University Extension Service Yazoo Mississippi Delta Joint Management District 


\section{Missouri}

Missouri Department of Natural Resources

\section{Montana}

Montana Board of Oil and Gas Conservation

Montana Department of Natural Resources and Conservation

\section{Nebraska}

Central Platte Natural Resources District

Lewis and Clark Natural Resources District

Lower Elkhorn Natural Resources District

Lower Loup Natural Resources District

Lower Platte North Natural Resources District

Lower Platte South Natural Resources District

Lower Republican Natural Resources District

Middle Niobrara Natural Resources District

Middle Republican Natural Resources District

Nebraska Department of Natural Resources

North Platte Natural Resources District

South Platte Natural Resources District

Tri-Basin Natural Resources District

Twin Platte Natural Resources District

Upper Big Blue Natural Resources District

Upper Elkhorn Natural Resources District

Upper Loup Natural Resources District

Upper Niobrara-White Natural Resources District

Upper Republican Natural Resources District

\section{Nevada}

Colorado River Commission

Federal Water Master

Nevada Department of Business and Industry

Nevada Department of Conservation and Natural Resources, Division of Environmental Protection

Nevada Department of Wildlife

Nevada Division of Water Resources

Southern Nevada Water Authority

\section{New Hampshire}

New Hampshire Department of Environmental Services, Water Management Bureau

\section{New Jersey}

New Jersey Department of Environmental Protection

\section{New Mexico}

New Mexico Office of the State Engineer-Water

Conservation Bureau

New Mexico Oil Conservation Division

\section{New York}

New York City Department of Environmental Protection

New York State Department of Environmental Conservation New York State Department of Health

\section{North Carolina}

North Carolina Department of Agriculture and Consumer Services, Agribusiness and Aquaculture

North Carolina Department of Environmental Quality, Water Supply Planning Branch

\section{North Dakota}

North Dakota Department of Mineral Resources North Dakota State Department of Commerce North Dakota State Water Commission

\section{Ohio}

Ohio Department of Natural Resources

\section{Oklahoma}

Oklahoma Corporation Commission

Oklahoma Water Resources Board

\section{Oregon}

Oregon Health Authority Drinking Water Services Oregon Water Resources Department

\section{Pennsylvania}

Delaware River Basin Commission

Pennsylvania Department of Environmental Protection

\section{Puerto Rico}

Puerto Rico Aqueduct and Sewer Authority

Puerto Rico Department of Health

Puerto Rico Department of Natural and Environmental Resources

Puerto Rico Electric and Power Authority 


\section{Rhode Island}

Rhode Island Department of Environmental Management Rhode Island Department of Health

Rhode Island Water Resources Board

\section{South Carolina}

South Carolina Department of Health and Environmental Control

\section{South Dakota}

South Dakota Department of Environment and Natural Resources

\section{Tennessee}

Tennessee Department of Environment and Conservation, Division of Water Resources

\section{Texas}

Texas Commission on Environmental Quality

Texas Railroad Commission

Texas Water Development Board-Water Use and Projections \& Planning

\section{U.S. Virgin Islands}

U.S. Virgin Islands Department of Planning and Natural Resources

\section{Utah}

Golf Course Superintendents Association of America State of Utah Automated Geographic Reference Center Utah Department of Agriculture and Food

Utah Department of Natural Resources, Division of Oil, Gas, and Mining

Utah Department of Natural Resources, Division of Water Resources

Utah Department of Natural Resources, Division of Water Rights

Utah Department of Natural Resources, Division of Wildlife Resources

Ute Tribe Fish and Wildlife Department

\section{Vermont}

Vermont Agency of Natural Resources, Department of Environmental Conservation, Drinking Water and Groundwater Protection Division

Vermont Fish and Wildlife Department, Agency of Natural Resources

\section{Virginia}

Virginia Department of Environmental Quality, Water Supply Planning Program

\section{Washington}

Golf Course Superintendents Association of America Washington State Department of Ecology

Washington State Department of Health

\section{West Virginia}

West Virginia Department of Environmental Protection, Water Use Section

West Virginia Public Service Division

\section{Wisconsin}

Public Service Commission of Wisconsin-Energy Division Public Service Commission of Wisconsin-Water Division Wisconsin Department of Administration (DOA)

Demographic Services Center

Wisconsin Department of Natural Resources

\section{Wyoming}

Wyoming Oil and Gas Conservation Commission Wyoming Water Development Commission 

For additional information, contact:

U.S. Geological Survey

National Water Use Science Project Team

wu-info@usgs.gov

or visit our website at:

https://water.usgs.gov/watuse/

Publishing support provided by the USGS West Trenton and Reston Publishing Service Centers 
